\title{
An Adaptive Battery Monitoring System for an Electric Vehicle
}

A Thesis submitted in partial fulfilment of the requirements for the Degree of Master of Engineering (Electrical and Electronic)

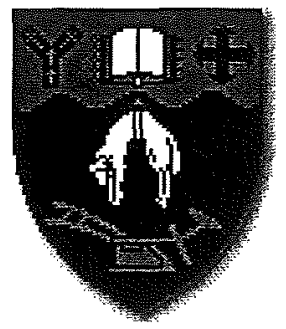

at the University of Canterbury

Christchurch, New Zealand

By Paul Grant Sinclair, B.E. (Hons)

March 1998 
In electric vehicles it is important to know the state of charge of the batteries in order to prevent vehicle strandings and to ensure that the full range of the vehicle is exploited. It is also useful to know state of health information about the batteries in the battery bank. This information can be used to predict when the batteries need replacing and can also identify batteries that are not performing optimally within the battery bank.

This thesis describes a battery monitoring system that is able to calculate the state of charge and state of health of multiple batteries in a battery bank. It has been designed specifically to monitor lead-acid batteries in an electric car environment using noninvasive measurement techniques. The monitor incorporates an adaptive monitoring method, which is based on coulometric measurements when the batteries are under load and predicted open circuit voltage measurements under no-load conditions.

The battery monitor is microcontroller based and uses remote battery monitoring modules to make the necessary battery measurements. Information is presented to the user of the car in the form of a state of charge meter on the instrument panel, similar to a fuel gauge in a conventionally power vehicle, and an alphanumeric LCD panel on the car's dashboard. . Aspects of both the monitor hardware and software are considered in this thesis.

Results obtained from bench tests of the monitor are presented which are followed by an evaluation of the monitor's performance. Consideration is also given to possible future improvements to the monitoring system. 


\section{Acknowledgments}

There are a number of people who have assisted me over the duration of this project. Firstly thanks should go to my supervisor Dr Richard Duke for his patience, guidance and advice throughout my project. Dr Simon Round also deserves thanks for his advice and work attempting to get the electric car operational.

My thanks go also to Ron Battersby and Ken Smart for their technical assistance and work on the electric car. I would also like to thank Dermot Sallis for manufacturing the many PCBs for this project and Warwick Earl for his assistance with equipment for the low temperature battery testing.

In would like to thank the members of power electronics postgraduate group, particularly Dave Ingram, Arnold Memelink, Jan Wiik, Adam Taylor and Hamish Laird, for their good advice and for providing a good working environment in the lab.

Finally I would like to thank my flatmates for putting up with me throughout last year and my parents for their support. I should specially thank my Father for his assistance with some of the photos in this thesis and my Mother for proofreading of this thesis. 


\section{Table of Contents}

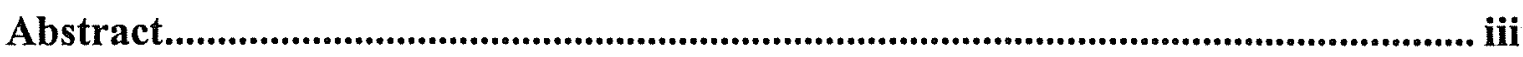

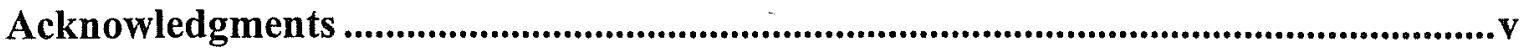

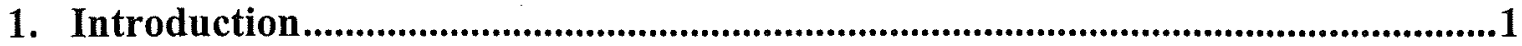

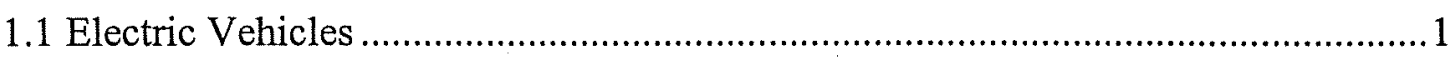

1.2 The Electrical Engineering Department's Electric Car.........................................

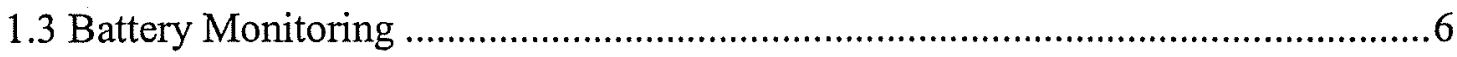

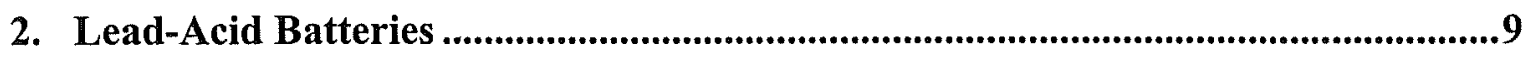

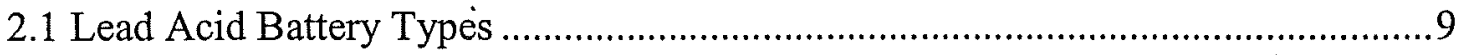

2.2 Sealed Lead-Acid Batteries............................................................................ 10

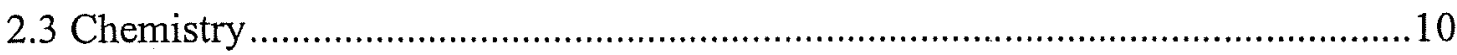

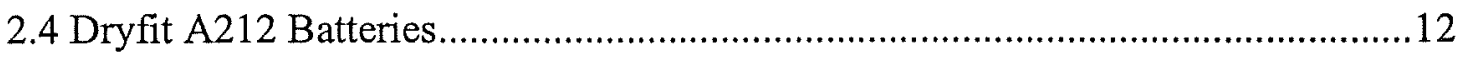

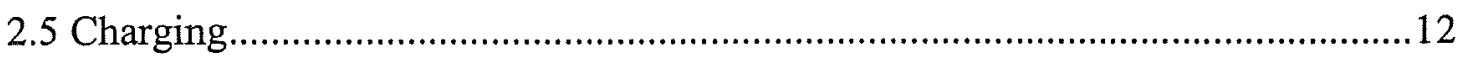

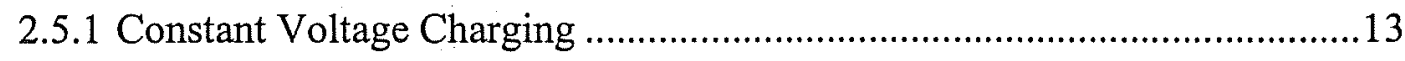

2.5.2 Constant Current Charging ................................................................. 14

2.5.3 Constant Current-Constant Voltage Charging.........................................15

3. Sealed Lead-Acid Battery Characteristics..............................................................17

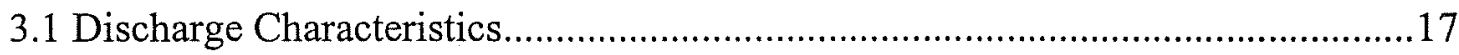

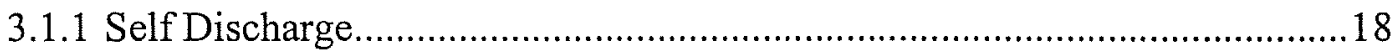

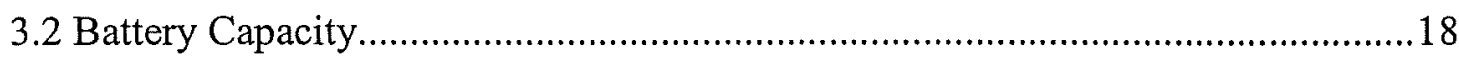

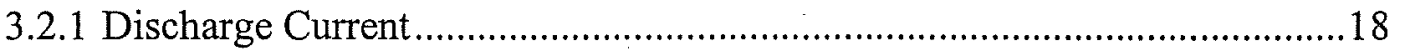

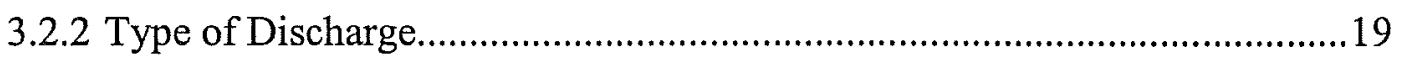

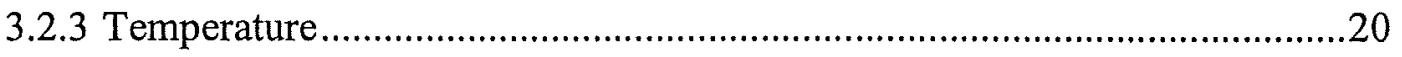

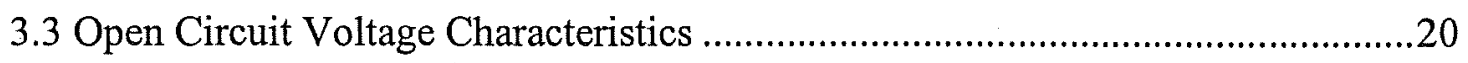

3.3.1 Open Circuit Voltage Relation to State of Charge .....................................21

3.3.2 Open Circuit Voltage Recovery Characteristics........................................21

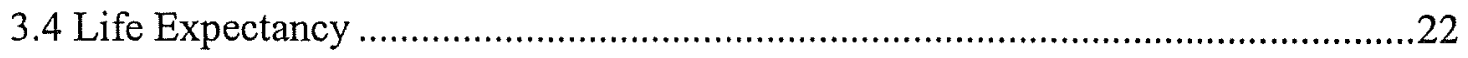

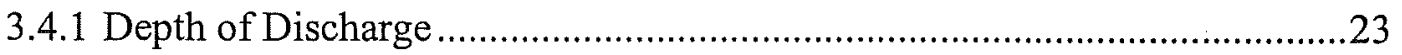

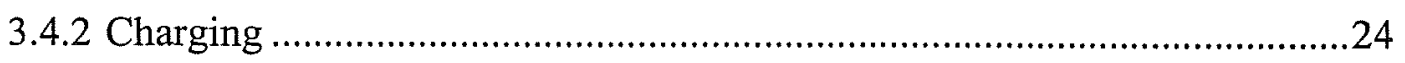

4. Battery Monitoring Techniques ..........................................................................225

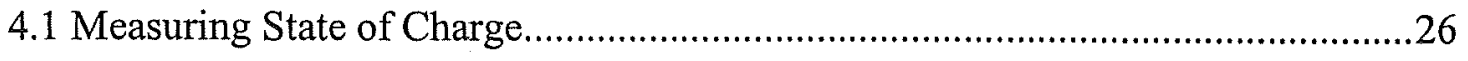

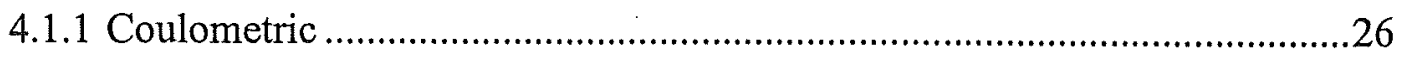

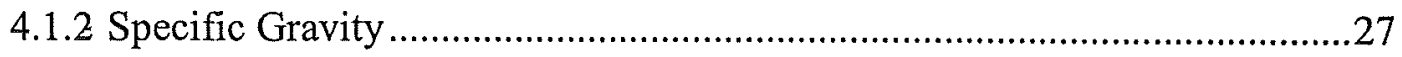

4.1.3 Open Circuit Voltage Measurements...........................................................2. 28

4.1.4 Loaded Voltage Measurements ...............................................................28

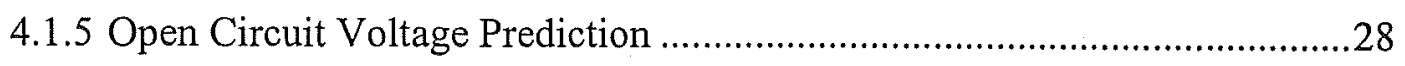

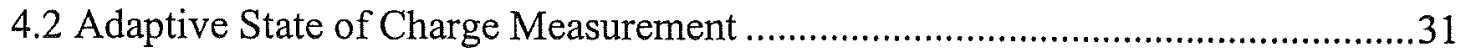




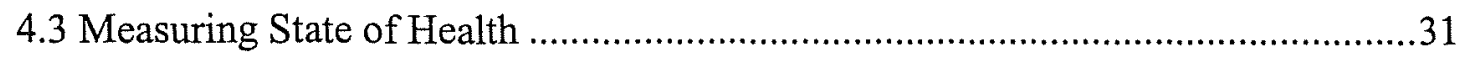

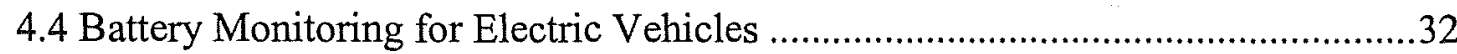

5. System Hardware

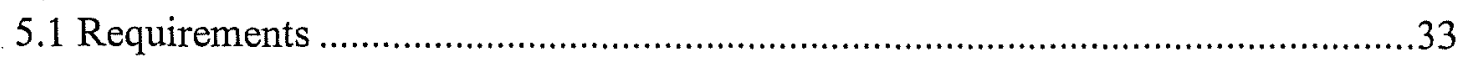

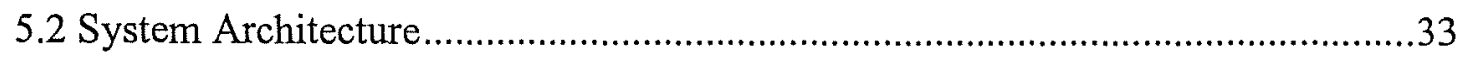

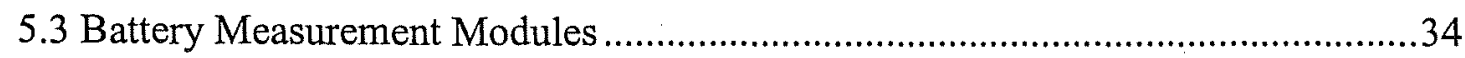

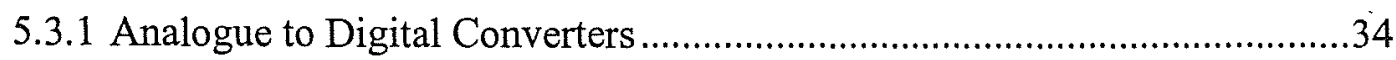

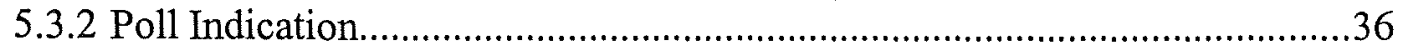

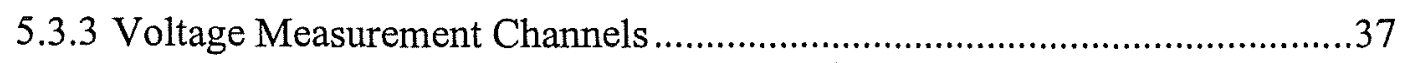

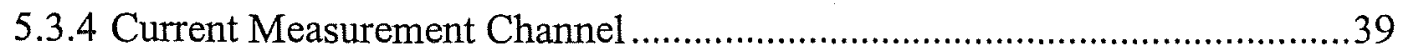

5.3.5 Temperature Measurement Channels .....................................................4 41

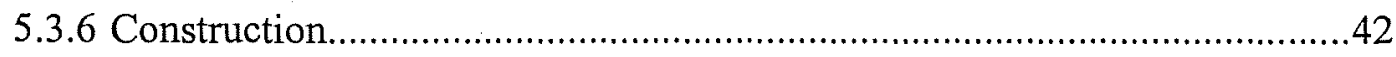

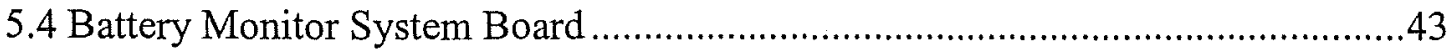

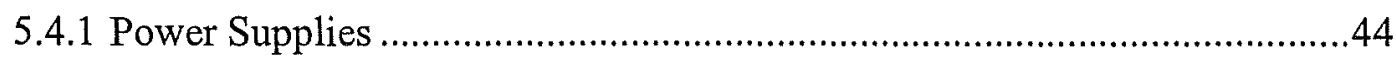

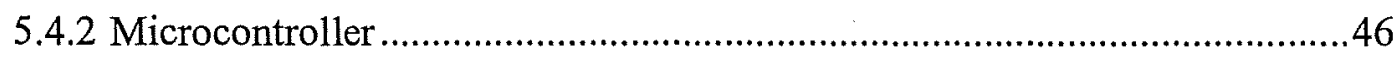

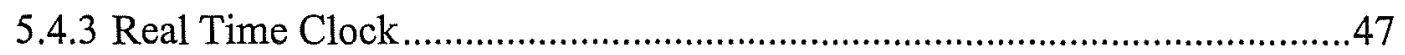

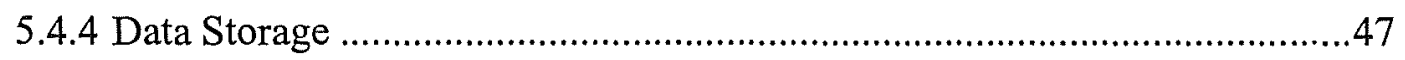

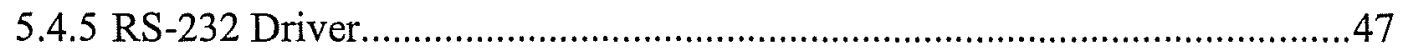

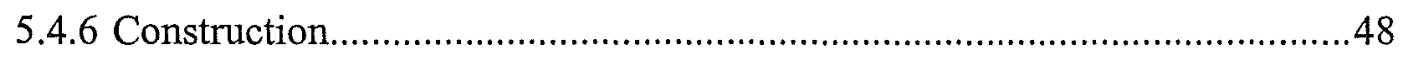

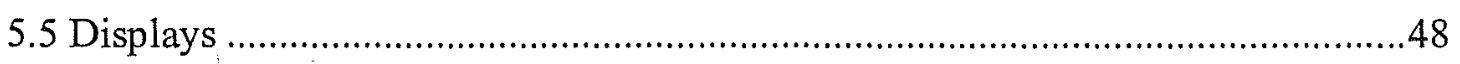

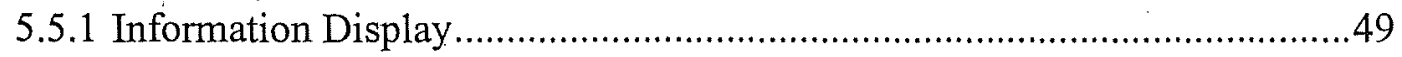

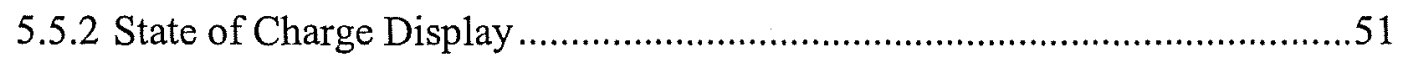

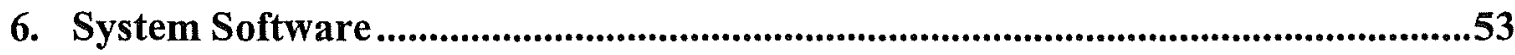

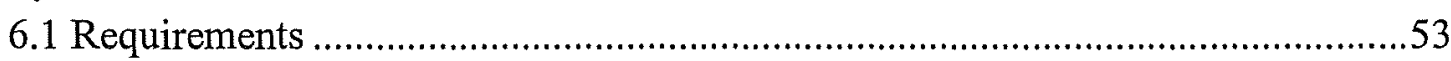

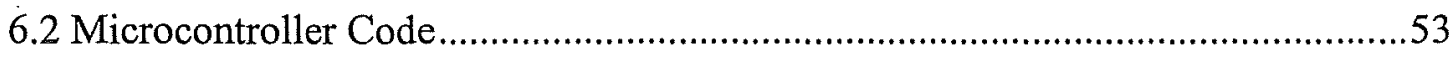

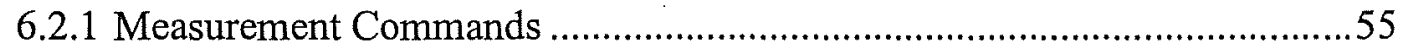

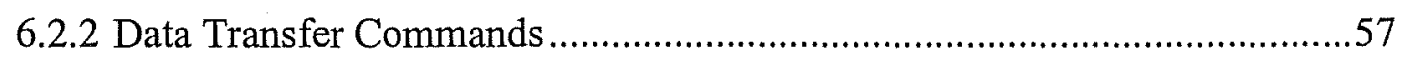

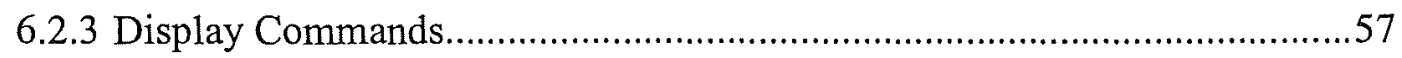

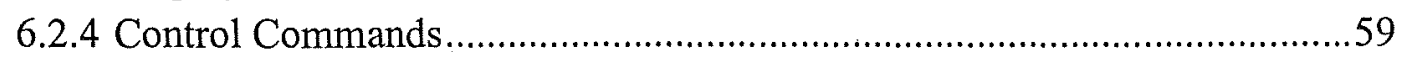

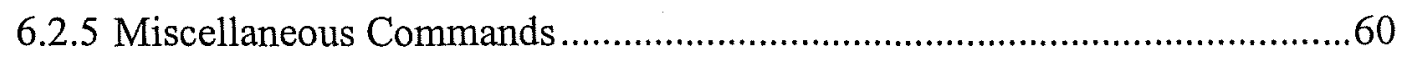

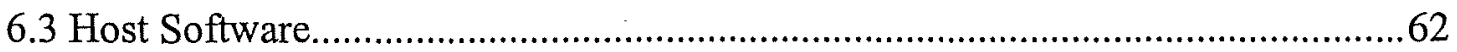

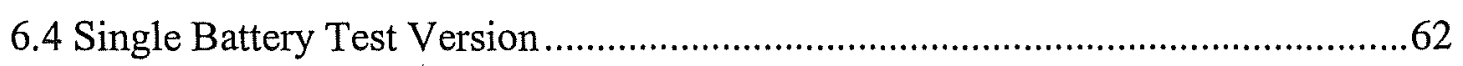

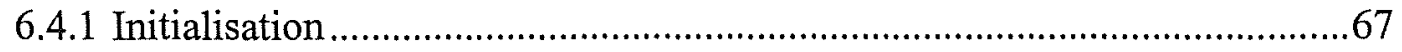

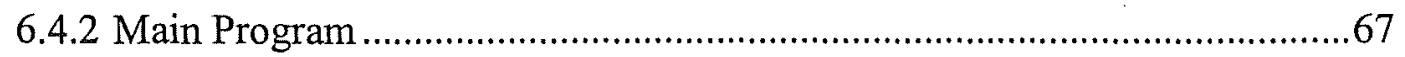

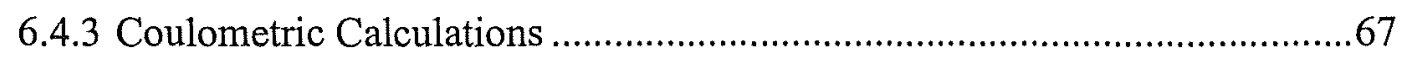

6.4.4 Open Circuit Voltage Calculations...........................................................69

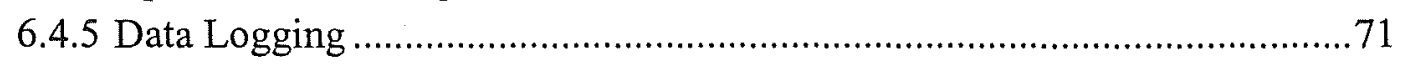

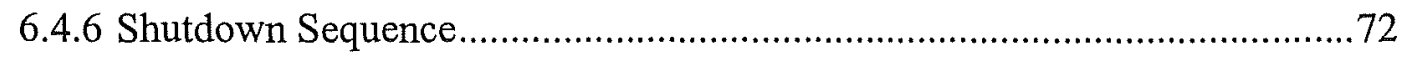

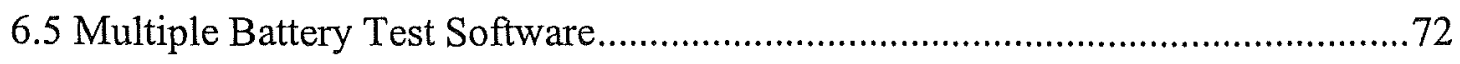

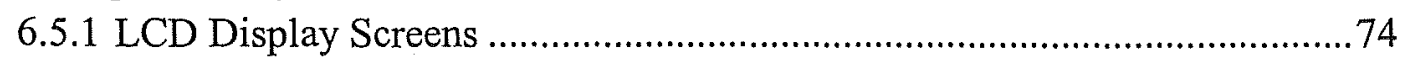

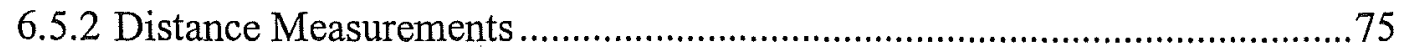




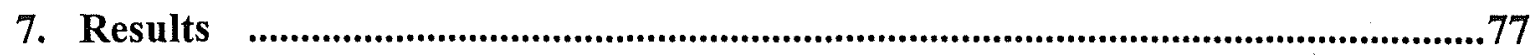

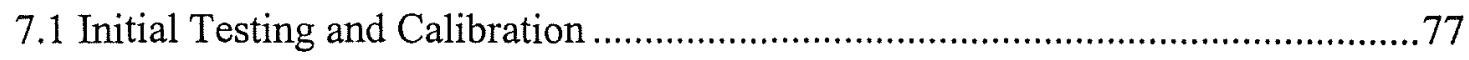

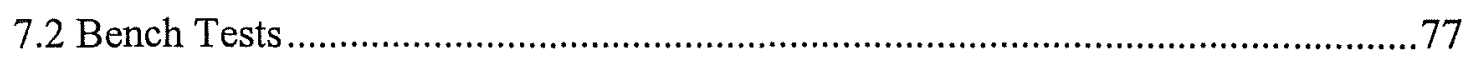

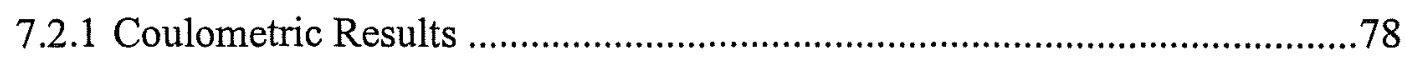

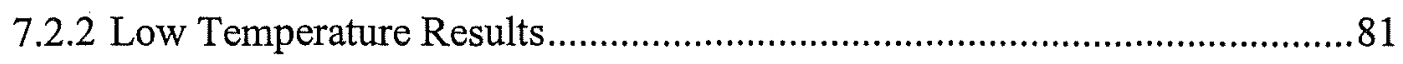

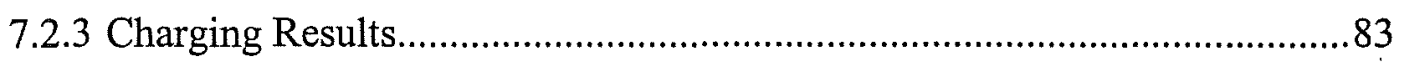

7.2.4 Open Circuit Voltage Results ............................................................. 84

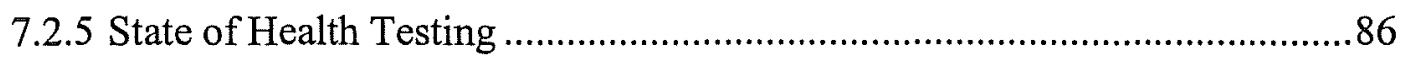

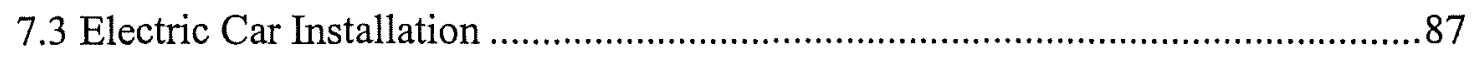

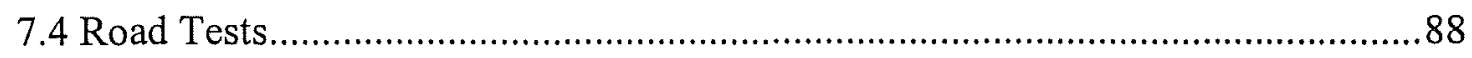

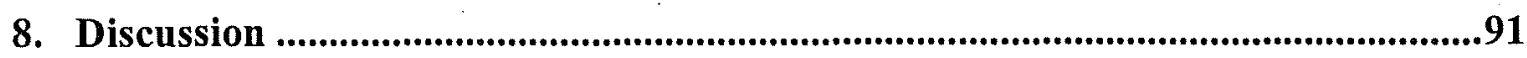

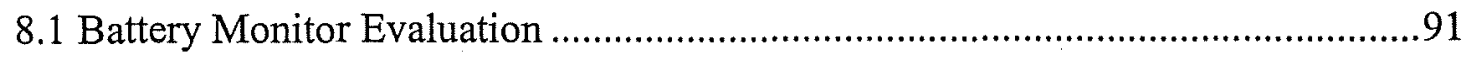

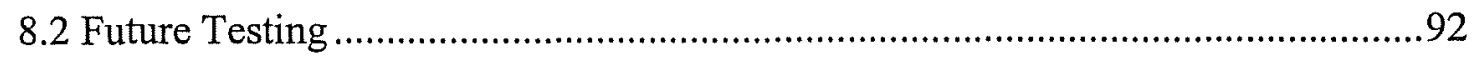

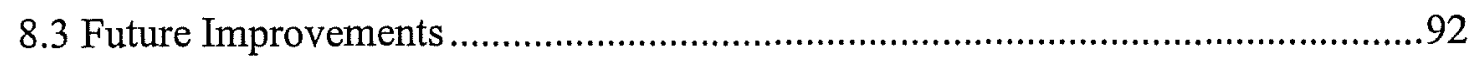

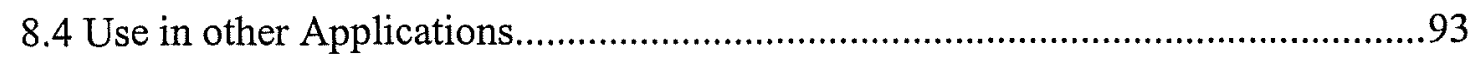

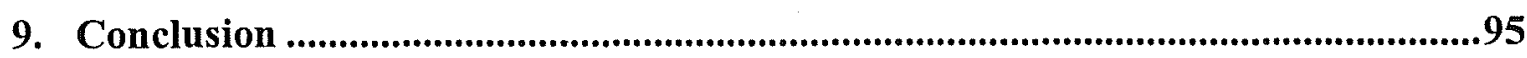

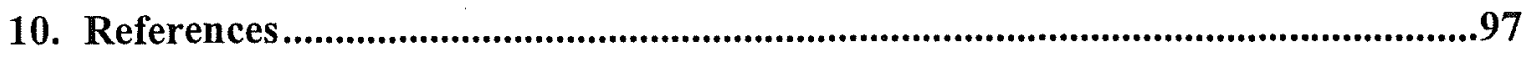

Appendix A. Battery Data Logger ..............................................................................99

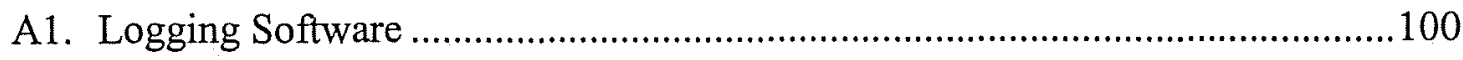

A2. Constant Current Discharger .................................................................. 101

Appendix B. Circuit Schematics .............................................................................105

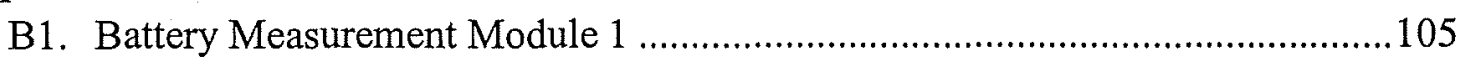

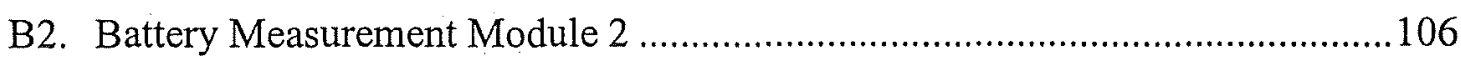

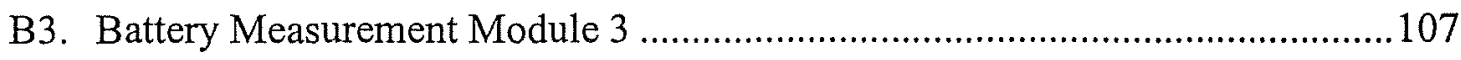

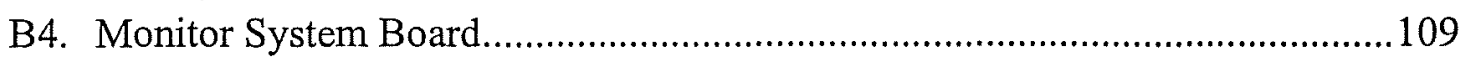

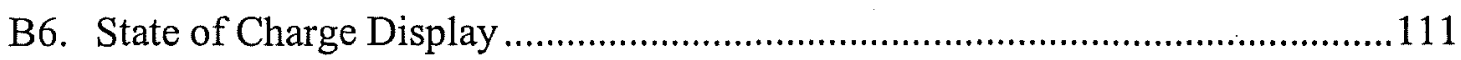

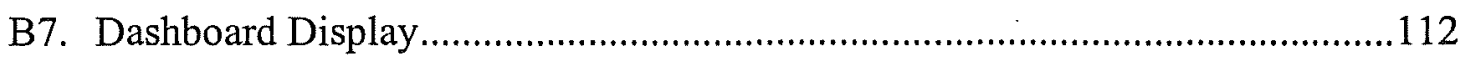

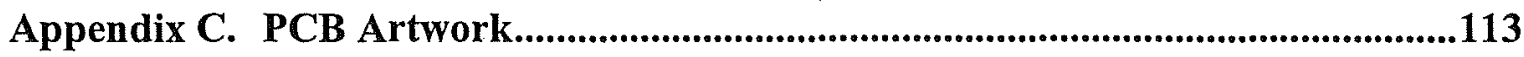

C1. Battery Measurement Module 1 ................................................................113

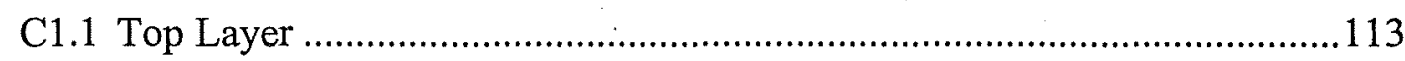

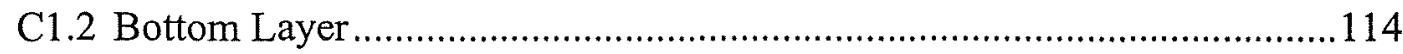

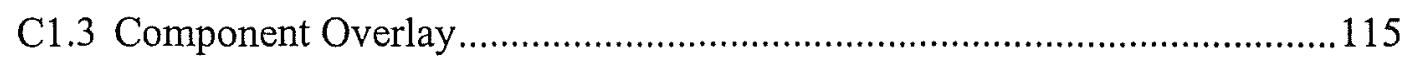

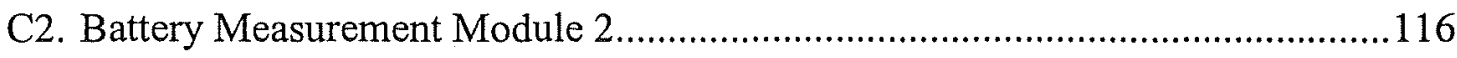

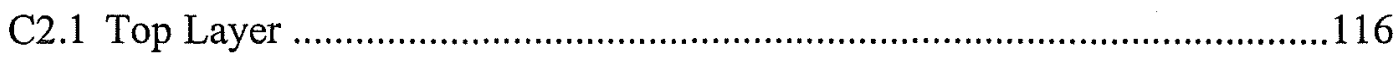

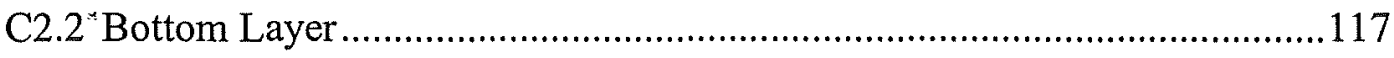

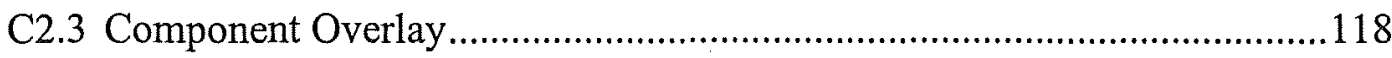

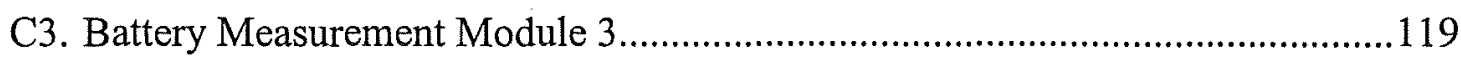

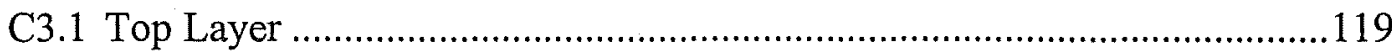

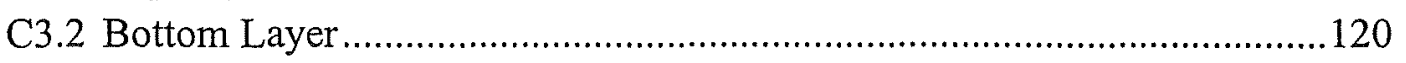




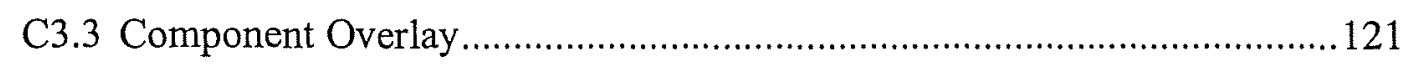

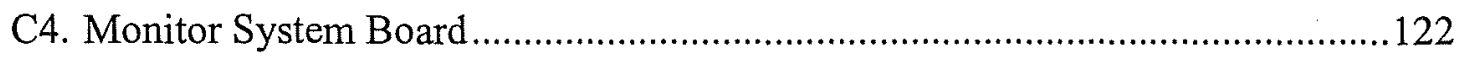

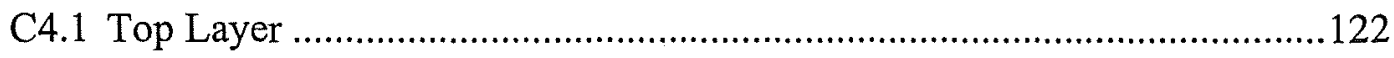

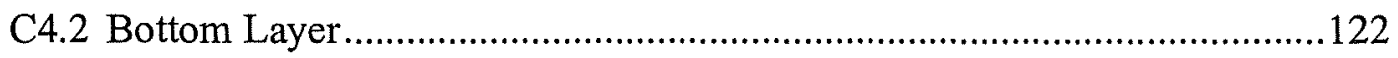

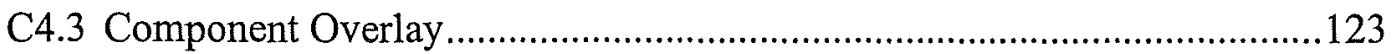

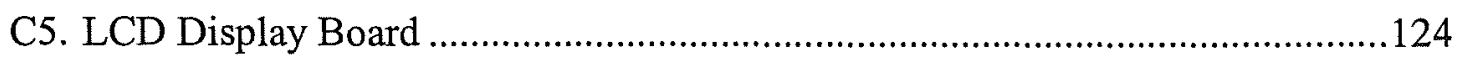

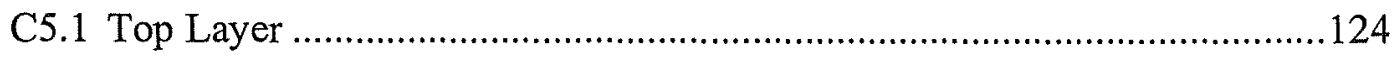

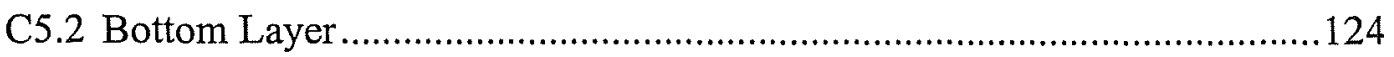

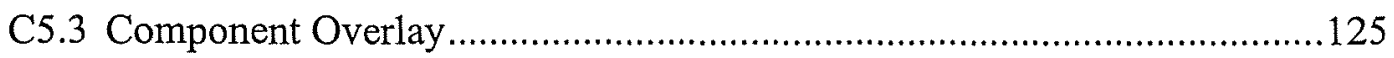

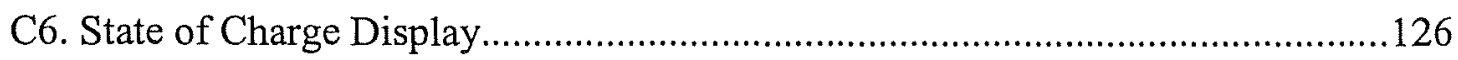

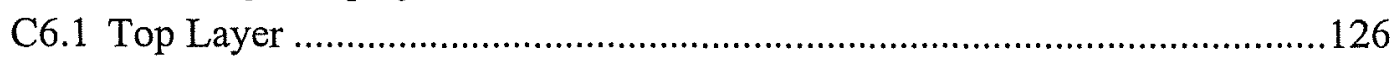

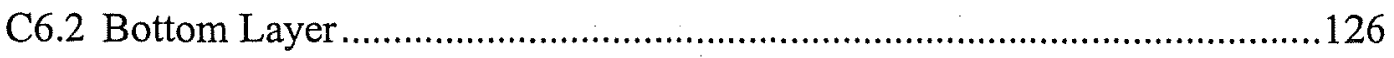

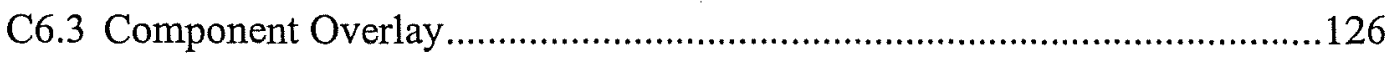

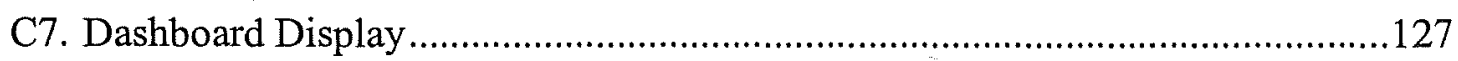

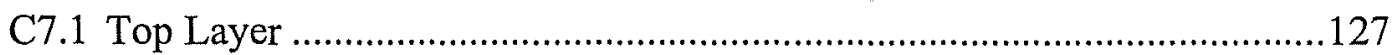

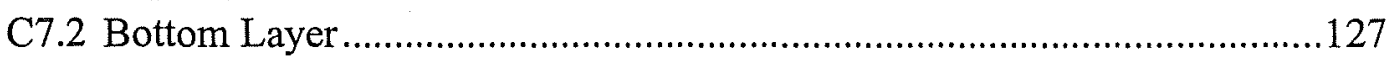

C7.3 Component Overlay......................................................................128

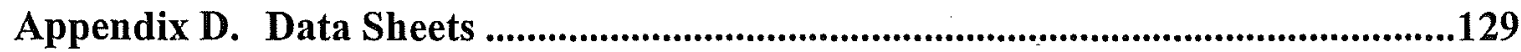

D1. Dryfit A200/A300 Lead-Acid Battery Specifications.......................................129

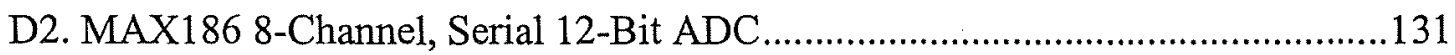

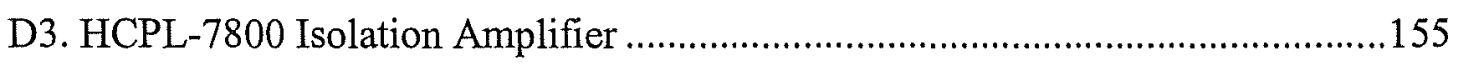

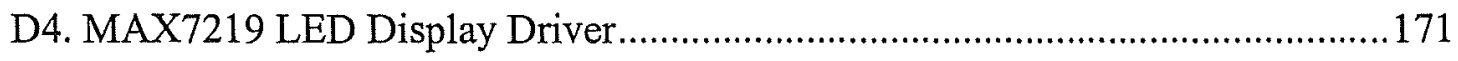

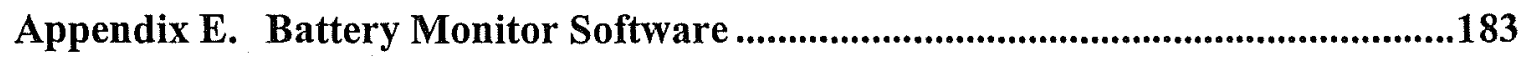




\section{Table of Figures}

Figure 1.1: An example of an early electric vehicle - a 1912 Walker half-ton electric truck owned by the MED........................................................................ 1

Figure 1.2: GM's EV1 electric car in the process of being recharged. .........................2

Figure 1.3: University of Cnaterbury's Mark II electric car. .................................... 4

Figure 1.4: Layout of the Mark II electric car. ..................................................... 4

Figure 1.5: Eight batteries are located under the bonnet of the electric car. ..................5

Figure 1.6: One of the battery compartments under the front seats containing six

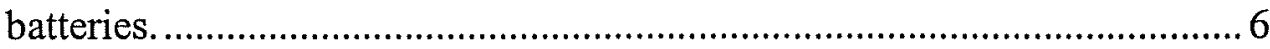

Figure 2.1: One of the batteries used in the electric car. ......................................... 12

Figure 2.2: Constant voltage charging characteristic............................................. 13

Figure 2.4: Constant current charge characteristic ............................................... 15

Figure 2.5: Constant current - constant voltage charge characteristic........................ 16

Figure 3.1: The discharge characteristic of a 36Ah A212 dryfit battery at constant

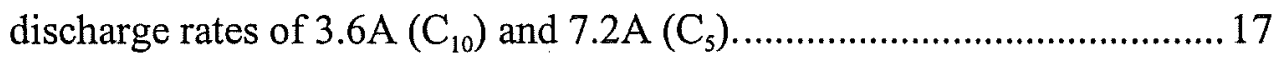

Figure 3.2: The capacity of a 36Ah A212 dryfit battery in relation to the discharge current. ............................................................................................ 19

Figure 3.3: Battery capacity variation in accordance with temperature for a dryfit battery. ....................................................................................... 20

Figure 3.4: Relationship between open circuit voltage and state of charge for an A212 dryfit battery............................................................................. 21

Figure 3.5: Open circuit voltage recovery curves measured on an A212 dryfit battery after discharges to $80 \%$ and $40 \%$ of remaining capacity at $36 \mathrm{~A} . .22$

Figure 3.6: The relationship between DOD and cycle life for a typical sealed leadacid battery.

Figure 4.1: Approximation of the open circuit recovery characteristic by two

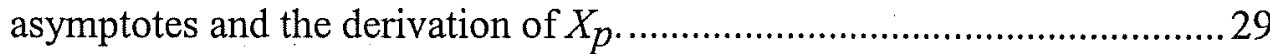

Figure 4.2: $\quad$ Open circuit recovery characteristic after a charge cycle. .......................... 30

Figure 5.1: Block diagram of the battery monitor hardware ...................................... 34

Figure 5.2: Circuitry associated with the MAX186 on the BMMs..............................35

Figure 5.3: The pulse stretching circuit used to extend the chip select line pulse so that it can give a meaningful visual indication of the BMM being polled. 36

Figure 5.4: Block diagram showing the signal processing components of a voltage

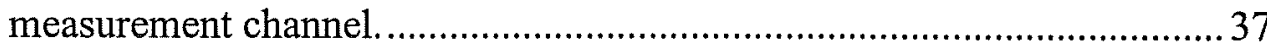

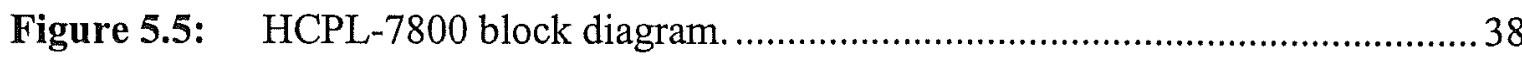

Figure 5.6: Circuit diagram of a single voltage measurement channel input and isolation circuitry. 38

Figure 5.7: The Hall effect current transducer used for current measurement in the

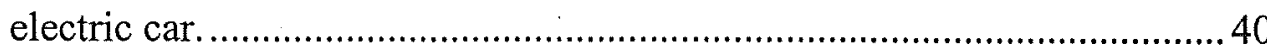

Figure 5.8: Block diagram of the current sensing and associated signal processing.... 40 
Figure 5.9: Current channel processing circuitry. ................................................ 41

Figure 5.10: Block diagram of the BMM temperature measurement channel. ............. 41

Figure 5.11: The temperature channel circuitry...................................................... 42

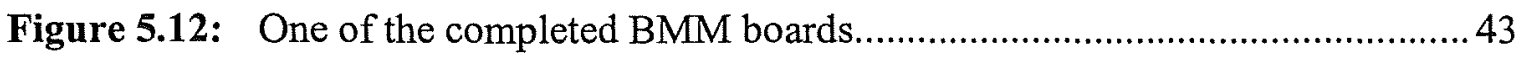

Figure 5.13: Block diagram of the battery monitor system board................................. 44

Figure 5.14: The battery monitor power supply circuitry ........................................... 45

Figure 5.15: The completed battery monitor system board....................................... 48

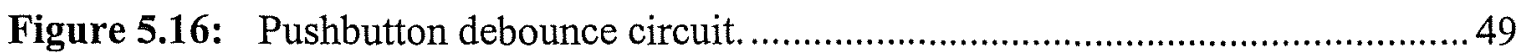

Figure 5.17: The front LCD display board with the LCD panel in place........................50

Figure 5.18: The rear of LCD display board where most of the circuitry is located......50

Figure 5.19: Circuit diagram of the state of charge display board. .............................. 51

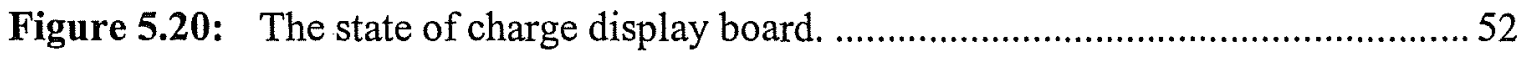

Figure 6.1: The basic program flow of the microcontroller code................................ 54

Figure 6.2: The main window of the battery monitor single battery test version. ....... 62

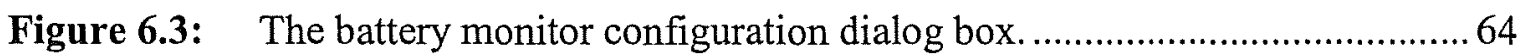

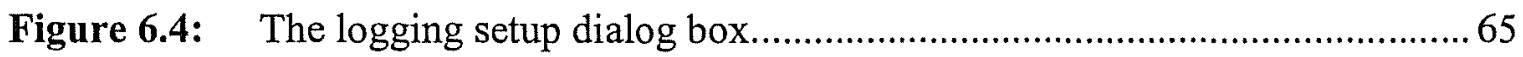

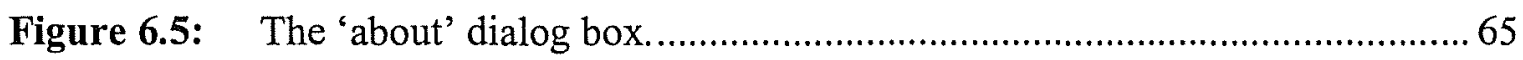

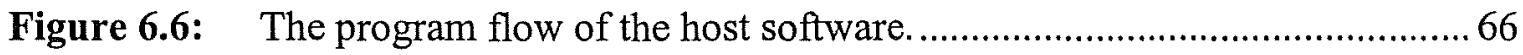

Figure 6.7: The program flow of the OCV calculation subroutine in the host

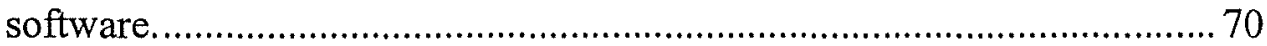

Figure 6.8: The main window of the multiple battery host software. ......................... 73

Figure 6.9: The battery monitor configuration dialog box for the multiple battery

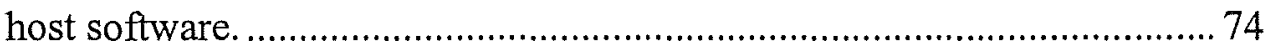

Figure 7.1: The results logged by the battery monitor during a 7.2A discharge cycle. 78

Figure 7.2: The insulated container and temperature controlling unit used to perform the low temperature battery monitor testing. ............................. 81

Figure 7.3: The results logged by the battery monitor during a charge cycle............. 83

Figure 7.4: One of the BMMs shown mounted in the electric car. The current sensor is also visible to the left of the BMM box. ................................... 87

Figure 7.5: The LCD display mounted in the car.................................................. 88

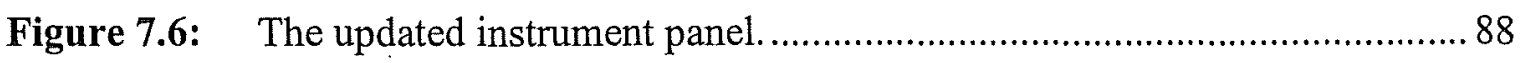

Figure A1: Block diagram of the battery data logging system.................................99

Figure A2: The logging system in operation................................................... 100

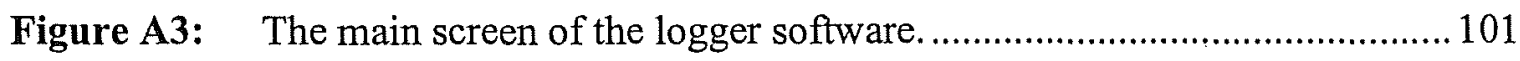

Figure A4: A constant current sink based around an LM317 voltage regulator. ....... 102

Figure A5: The final circuit of the discharger and switching relays....................... 102

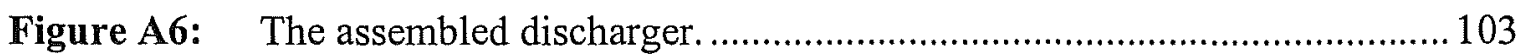




\section{Table of Tables}

Table 6.1: Summary of the battery monitor measurement commands. ..........................56

Table 6.2: Summary of the battery monitor data transfer commands............................57

Table 6.3: Summary of the battery monitor display commands...................................5 58

Table 6.4: Summary of the battery monitor control commands...................................5 59

Table 6.5: Summary of the remaining battery monitor commands. ................................ 61

Table 6.6: Summary of the battery monitor LCD display screens. .............................. 75

Table 7.1: A comparison of time based and coulometric SOC results during a discharge at a rate of 7.2A.................................................................... 79

Table 7.2: Coulometric results during a 18A discharge test........................................ 80

Table 7.4: Coulometric results during a 54 discharge test.......................................... 80

Table 7.3: Coulometric results during a 36A discharge test........................................ 80

Table 7.5: Coulometric results during a 72A discharge test....................................... 80

Table 7.6: The average error in each of the five discharge tests................................. 81

Table 7.7: Results obtained from the low battery temperature tests............................. 82

Table 7.8: Open circuit voltage results obtained during a 7.2A discharge. .................... 85

Table 7.9: Open circuit voltage results obtained during a $18 \mathrm{~A}$ discharge......................85

Table 7.10: Open circuit voltage results obtained during a 54A discharge..................... 86 


\section{Introduction}

\subsection{Electric Vehicles}

Electric vehicles are by no means a new concept. In the early 1900s electric vehicles made up a significant proportion of the world's vehicle fleet (figure 1.1). At the time electric vehicles shared the roads with internal combustion engine and steam powered vehicles [1]. Improvements to the performance and reliability of internal combustion engines lead to the demise of electric vehicles within a decade. Battery powered vehicles were not able to compete with the greater speed and range offered by the internal combustion engine.

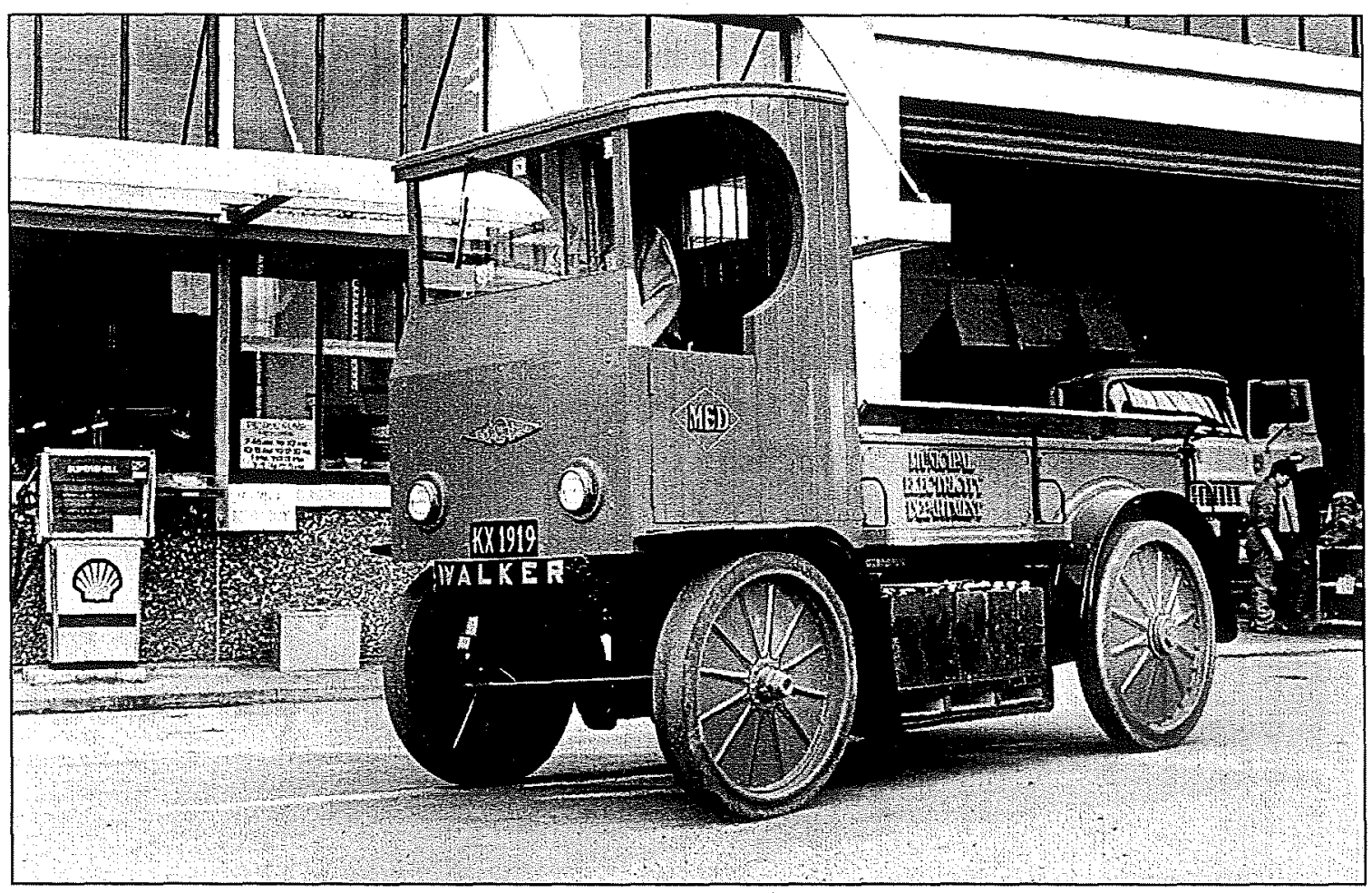

Figure 1.1: An example of an early electric vehicle - a 1912 Walker half-ton electric truck owned by the MED. - Roy Sinclair

The 1990s have seen a revival of interest in electrically powered vehicles due to increased environmental concerns. Vehicles based on the internal combustion engine are a major source of emissions that contribute to air pollution in urban areas [1]. Electric vehicles are seen as a preferred alternative as they produce no emissions while running. Electric vehicles are however not totally non-polluting as emissions may be generated in the production of electricity used to recharge their batteries. These emissions are still 
significantly less than those produced by an internal combustion engine powered vehicle. This is due to the fact that in many areas a significant proportion of electricity generation comes from non-polluting sources such as hydro or nuclear generation. Even if all the electricity used does come from thermal power stations the emissions produced are much lower due to the better efficiencies of thermal power stations compared to internal combustion engines. As power stations are generally located outside urban areas, their emissions do not generally contribute to urban air pollution levels. Thermal power stations also produce far less of the more noxious gases, such as carbon monoxide (CO), ozone and nitrogen oxides (NOx), produced by internal combustion engines [1]

The biggest push for electric vehicles has come from the California Air Resources Board. In October 1990, in an effort to reduce Los Angeles city's smog, the California Air Resources Board mandated that $2 \%$ of vehicles sold in California must be zero emission vehicles (ZEVs) by 1998 [2], although this now looks as if it will be delayed. In addition the number of ZEVs sold must rise to $5 \%$ in 2001 and $10 \%$ by 2003 . Other US states have since adopted the same standards and interest in European and Asian cities in improving air quality standards has created a large potential market for electric vehicles. This has led many of the world's car manufacturers to develop production electric vehicles.

The first modern car manufacturer to produce a commercial electric car was General Motors. In December 1996 GM released the EV1 (figure 1.2), a two seater electric car [3]. With an electronically governed top speed of $129 \mathrm{~km} / \mathrm{h}$ and an acceleration of 0 $100 \mathrm{~km} / \mathrm{h}$ in less than nine seconds this car offers performance that compares well with conventionally powered cars. The EV1 also includes many safety and comfort features including dual air bags, air conditioning and a CD player.

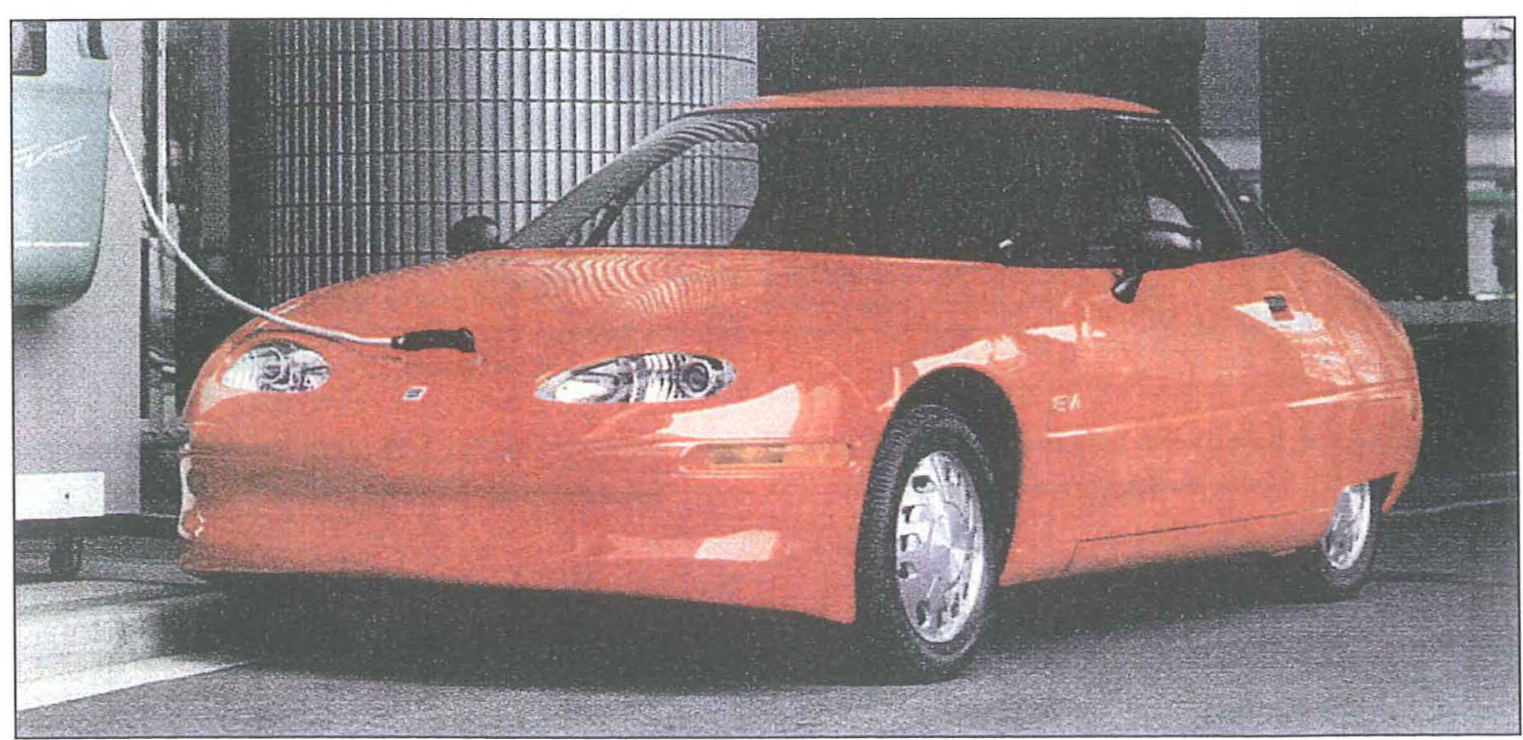

Figure 1.2: GM's EV1 electric car in the process of being recharged. - IEEE Spectrum, December 1996 
While electric vehicles offer the advantages of zero emissions, quiet running and increased reliability over internal combustion engine based vehicles, they are not without their problems. The main factor hampering their development is battery technology. This is due to the fact that even the best currently available batteries cannot store anywhere near the same amount of energy for a given weight as can be stored in liquid fuels. As a result the range of electric vehicles is generally significantly less than comparable conventionally powered vehicles. Another problem facing electric vehicles is the time that the batteries take to recharge compared to the time required to refill a fuel tank. These factors currently limit the uses of electric cars to commuting and other local trips rather than long range travel uses.

High costs are the other major problem facing electric vehicles. In order to get the best performance and range out of electric vehicles they must be designed to be very efficient. This means that they must be light in weight, have low drag coefficients and low rolling resistances, and have efficient motors and drive electronics. These factors make them more expensive to produce and this has led several car manufacturers to lease electric vehicles rather than sell them. It is also very likely that the batteries will need to be replaced over the lifespan of the car, further adding to the costs of operating an electric vehicle.

\subsection{The Electrical Engineering Department's Electric Car}

The University of Canterbury has been involved in electric car development, although on a much smaller scale than the large motor vehicle companies. The main motivation behind development of an electric car has been both to demonstrate that an electric powered car is viable and to provide a suitable test-bed for drive systems, control systems and various other systems.

Work began on the University of Canterbury's original electric car, the 'Mark I,' in 1974 and it ran for the first time in 1976. The car was driven by AC induction motors which at the time were rarely used in vehicles [4]. The car although successful, had a limited range of only $40 \mathrm{~km}$.

In order to improve this range a second car, the 'Mark II,' was developed between 1976 and 1982. This car is based on the body of a 1962 Austin A40 Farina with additional streamlining to reduce its drag (figure 1.3). Various other parts were sourced from other types of cars, including a VW and a Hillman Avenger. With its much improved aerodynamic characteristics, the range of the car improved to $60 \mathrm{~km}$ and a top speed of $80 \mathrm{~km} / \mathrm{h}$ could be obtained. 


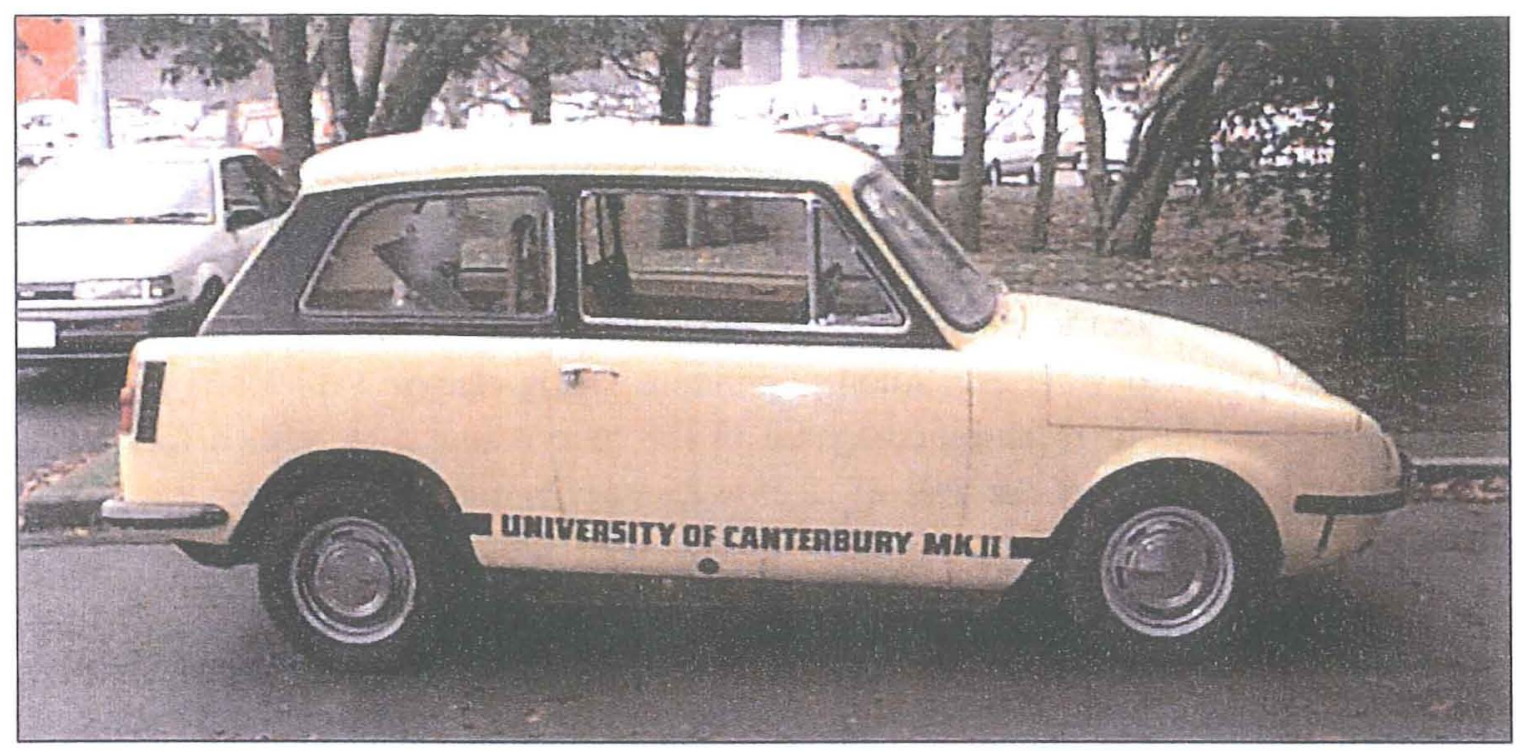

Figure 1.3: University of Canterbury's Mark II electric car.

Figure 1.4 shows the layout of the main components of the Mark II. Like the Mark I, the Mark II is driven by two conventional three-phase AC induction motors linked to the two rear wheels by chain drives. The induction motors are of standard $2.2 \mathrm{~kW}$ frame size. They have been specially wound to allow them to operate off a lower supply voltage and to enable them to run at up to three times rated torque and speed. They are capable of developing a maximum continuous power of $6 \mathrm{~kW}$ and can develop up to $11 \mathrm{~kW}$ for short periods.

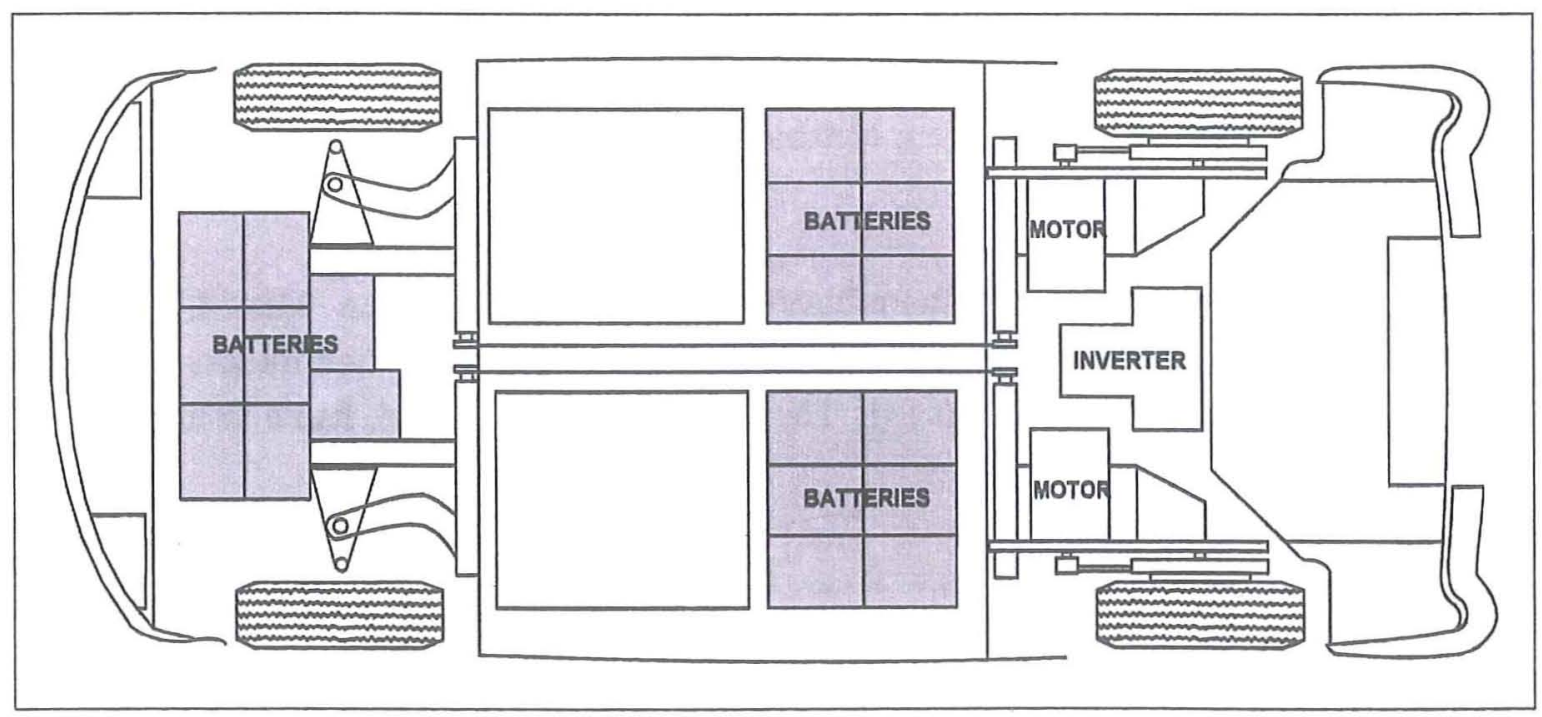

Figure 1.4: Layout of the Mark II electric car [4].

Power to the motors is provided by a three-phase inverter located between the two motors. The original inverter used in the car was an analogue controlled, thyristor based PWM inverter. In 1984, after the car had been withdrawn from service, work began on a new inverter based on bipolar junction transistors. The analogue control system was replaced 
with a modified microcontroller based GEC drive controller. In 1996 the car received a new set of batteries and ran again for the first time in twelve years.

Due to reliability problems and the lack of software support, the GEC controller was replaced with a PDL drive controller originally designed as part of a commercial PDL motor drive. Work is also currently being undertaken to replace the inverter with one based on more modern and more efficient IGBT devices.

The car is powered by twenty series connected $12 \mathrm{~V}$ lead acid batteries to give a nominal 240V DC bus. The batteries are of the sealed gel type and are each rated at 36Ah. Sealed batteries were chosen for this application as they are maintenance free and, since the electrolyte in them is a gel, there is no likelihood of electrolyte splashing around and spilling. Eight of the twenty batteries are located under the bonnet and the remaining twelve are located in two groups of six under the two front seats (figures 1.5 and 1.6). An on board charger is used to charge the batteries from a standard wall socket.

The car has regenerative braking which allows energy to be returned to the batteries during braking and hence improves the range of the car. Regenerative braking also removes the need for conventional mechanical brakes on the rear wheels. Hydraulic brakes are retained on the front wheels for safety and to comply with relevant vehicle regulations.

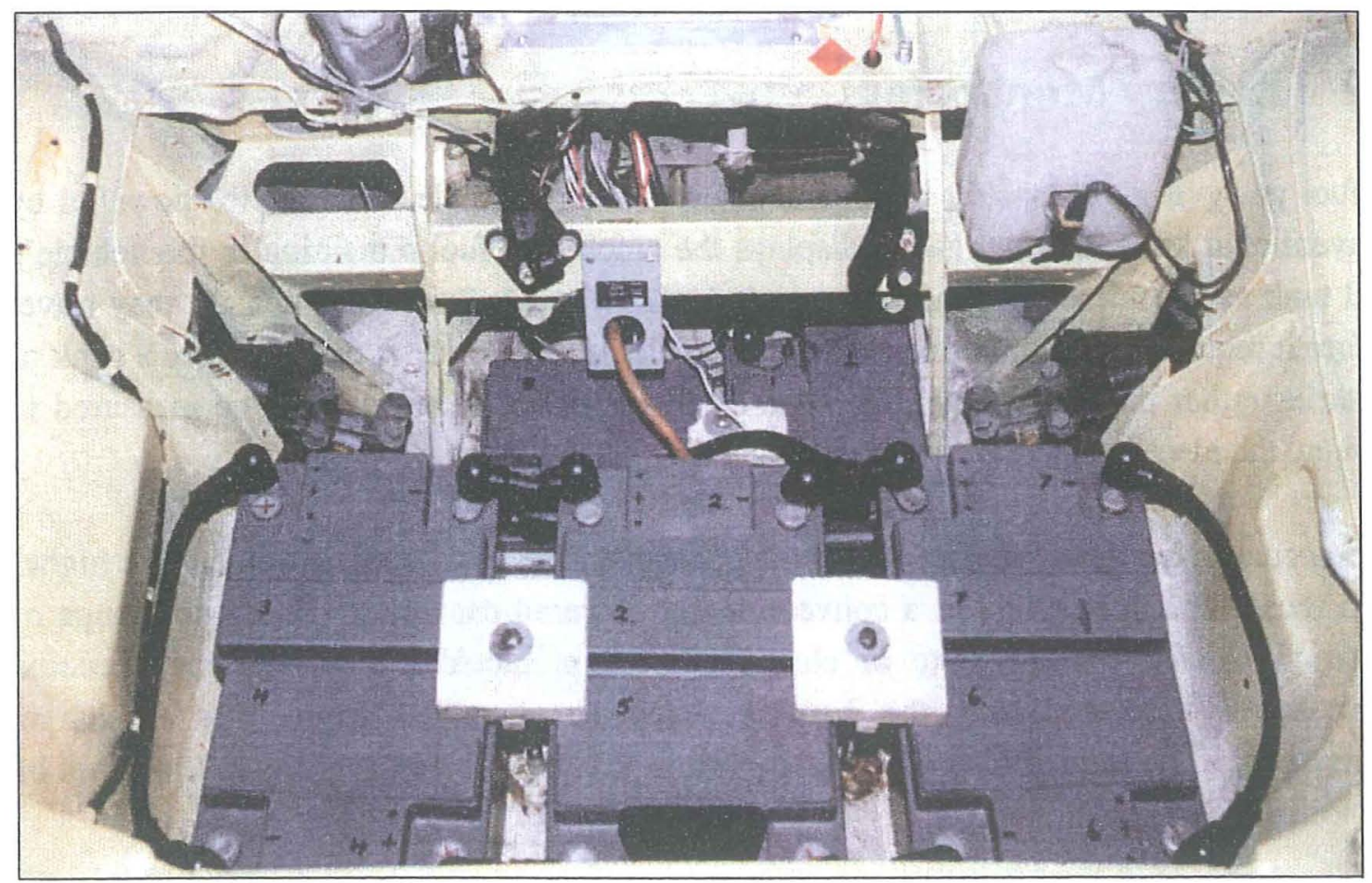

Figure 1.5: Eight batteries are located under the bonnet of the electric car. 


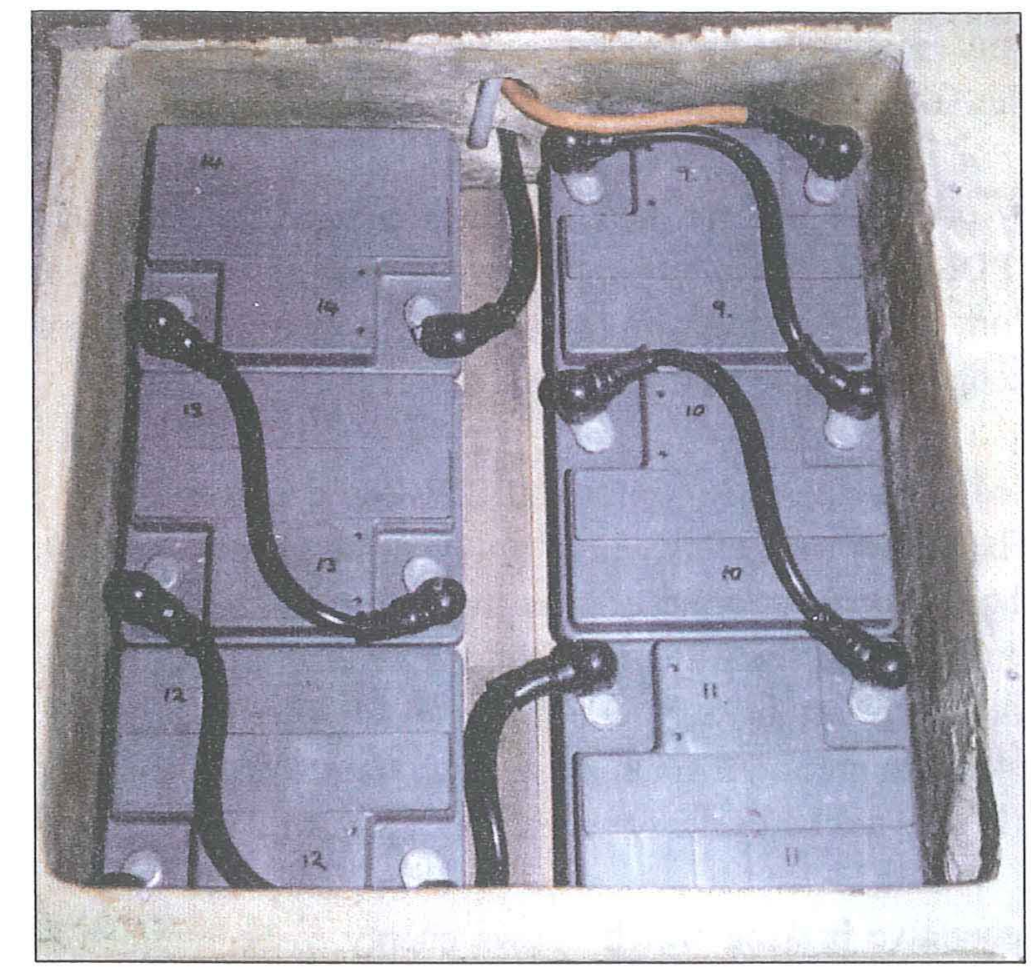

Figure 1.6: One of the battery compartments under the front seats containing six batteries.

Power for the $12 \mathrm{~V}$ electrical components in the car's electrical system such as the lights, windscreen wipers and horn is provided by an auxiliary power supply. This is based around a 350W DC-DC converter.

\subsection{Battery Monitoring}

A fuel gauge is a reasonably important device for any vehicle. In vehicles powered by conventional fuels, the fuel gauge displays the amount of fuel remaining in the vehicle's fuel tank and hence allows the driver to judge how much further the vehicle may travel before it requires refuelling. In an electric vehicle, where power is derived from a bank of batteries rather than liquid fuel, the state of charge of the batteries must be measured to provide the electric vehicle's equivalent of a fuel gauge.

The accuracy requirements from a state of charge meter in an electric vehicle are higher than those for a fuel gauge in a conventionally powered car due to the limited range of electric vehicles. If the state of charge is over predicted the vehicle may become unexpectedly stranded and the batteries may be damaged from over discharge. Conversely if the state of charge is under predicted the full range of the vehicle will not be exploited.

Whereas in a conventional vehicle the fuel gauge reading relates directly to a physical measurement, the level of fuel in the vehicle's tank, it is not as easy to obtain an equivalent measurement that relates to the state of charge of the batteries in an electric 
vehicle. Several battery parameters are typically used to calculate the state of charge. The parameters used should ideally be measured using non-invasive techniques to avoid having to modify the batteries.

Since batteries have a limited life, it is also useful to know what their state of health is so that it can be predicted when the batteries are likely to need replacing. If information on the state of health of individual batteries in the battery bank is measured, then weak batteries in the battery bank can be detected and action taken before those weak batteries become damaged or cause damage to the other batteries in the bank. This can occur due to the higher stresses placed on the other batteries in the bank when one or more batteries are not performing optimally.

This thesis looks at the development of a battery monitoring system for an electric vehicle that measures both the state of charge of the batteries and their state of health. It also offers several other features. These include battery parameter logging that allows the performance of the batteries under different driving conditions to be monitored and prediction of the remaining distance that the car can travel before requiring recharging to help avoid vehicle strandings.

Chapters two and three of this thesis give a general background and explain some of the characteristics of lead acid batteries used in the department's electric car. This is followed by a description in chapter four of techniques that can be applied for monitoring batteries of this type. Chapters five and six look at the implementation of the battery monitor from both a hardware and software point of view. Finally results from performance tests on the monitor are given in chapter seven. 


\section{Lead-Acid Batteries}

Lead-acid batteries have been in use for well over a century and are one of the most popular types of secondary battery. Their use continues to grow as new applications appear for battery power systems in energy storage applications, portable electronic devices and electric vehicles. These new applications have also lead to new development work to improve lead-acid battery performance.

The most popular application for lead-acid batteries is engine starting. Lead-acid batteries are also very popular in standby power systems, remote power systems and uninterruptible power supplies (UPS). As well as being used in electric vehicles they are also used in other traction applications such as forklifts, floor scrubbers, golf carts and mining vehicles.

There are many other types of batteries that have higher energy densities than lead-acid batteries making them more desirable for electric vehicle applications. However the only other sufficiently developed battery technology currently suitable for commercial use in electric vehicles is nickel-cadmium which suffers from the problems of high cost and the high toxicity of cadmium. Other battery technologies which may become viable alternatives in the near future include nickel-metal-hydrate, sodium-sulfide, lithium-iron and zinc-bromide [5]. These technologies all offer higher energy densities and longer cycle lives than lead-acid batteries.

Low cost is the most favourable feature of lead-acid batteries. Lead-acid batteries also perform well over a wide temperature range and offer reasonably good performance and cycle life. Since lead can be cheaply recycled they are not as environmentally unsound as some other battery technologies.

\subsection{Lead Acid Battery Types}

There are many tradeoffs in the design of lead-acid batteries. As a result, lead-acid batteries are designed so that their parameters are optimised for the particular application they are designed for. These parameters include power density, cycle life, service life and cost.

The most common type of lead-acid battery is the automotive SLI (starting, lighting and ignition) battery used for engine starting. This type of battery is designed to provide high currents for short periods to drive a starter motor and as a result are designed with low internal impedances. Once the engine is running the battery is recharged by the alternator 
driven from the engine and maintained at full charge until the engine is stopped again. SLI batteries are not designed for deep cycle discharges and may have their life shortened if this occurs.

Deep cycle lead-acid batteries are designed for maximum cycle life under deep discharge conditions. Deep-cycle batteries are popular in traction applications. In these applications they are used in a mobile environment where battery weight is important. For this reason they are generally also designed to have high energy densities. This is particularly important in electric vehicle applications where vehicle range is a major consideration.

The other main type of lead-acid battery is designed for use in standby power systems. In this type of applications service life under continuous float charge conditions is an important consideration, whereas battery size and weight considerations generally are not.

\subsection{Sealed Lead-Acid Batteries}

The sealed lead-acid (SLA) battery is one type of lead-acid battery that is becoming increasingly popular. As the name implies this type of battery is sealed and is therefore maintenance free since the electrolyte does not need to be topped up. They incorporate a recombination process which allows oxygen produced during charging to be returned to the electrolyte. A pressure release valve is incorporated into the design of the battery to release excess gas if the pressure builds up too high.

The valve-regulated lead-acid (VRLA) battery is one type of SLA battery. This type of battery is designed to vent at low pressures and the vent is used to keep the battery pressure within a certain range as opposed to fully sealed batteries which are designed only to vent at high pressures for safety reasons.

Since the electrolyte is immobilised, these types of battery can be operated in different orientations without spillage of the electrolyte occurring. This feature allows SLA batteries to be used in portable applications where the battery does not need to be kept upright. The electrolyte is immobilised either by absorbing it in a highly porous material used as the separator in the battery or by forming the electrolyte into a gel. In the later case the gel is formed usually by adding fumed silica to the electrolyte.

\subsection{Chemistry}

The reactions that occur in lead-acid battery cells occur between the lead electrodes and the sulphuric acid contained within the electrolyte. Lead dioxide is used for the positive electrode and metallic lead is used for the negative electrode. The electrolyte usually contains around $37 \%$ sulphuric acid by weight in a fully charged condition. Each cell has a nominal voltage of $2 \mathrm{~V}$. 
During discharge both electrodes are converted to lead sulphate and water is produced. On recharge the electrodes are converted back to lead and lead dioxide and the concentration of sulphuric acid increases. Chemical equation 2.1 describes the complete reaction which is also known as the double-sulphate reaction. When the cell is being discharged the reaction takes place from left to right and occurs in the reverse direction during charge.

$$
\mathrm{PbO}_{2}+\mathrm{Pb}+2 \mathrm{H}_{2} \mathrm{SO}_{4} \rightleftharpoons 2 \mathrm{PbSO}_{4}+2 \mathrm{H}_{2} \mathrm{O}
$$

The actual reactions occurring at each of the electrodes are described in equation 2.2 for the positive electrode and equation 2.3 for the negative electrode.

$$
\begin{aligned}
& \mathrm{PbO}_{2}+3 \mathrm{H}^{+}+\mathrm{HSO}_{4}^{-}+2 \mathrm{e} \rightleftharpoons 2 \mathrm{H}_{2} \mathrm{O}+\mathrm{PbSO}_{4} \\
& \mathrm{~Pb}+\mathrm{HSO}_{4}^{-} \rightleftharpoons \mathrm{PbSO}_{4}+\mathrm{H}^{+}+2 \mathrm{e}
\end{aligned}
$$

During charging, as the cells in the battery approach full charge and all of the $\mathrm{PbSO}_{4}$ has been converted back to $\mathrm{Pb}$ and $\mathrm{PbO}_{2}$, the cells begins to overcharge. The overcharge reactions involve the production of hydrogen and oxygen as the cell starts gassing. Gassing results in a loss of water in the electrolyte in an unsealed cell. Chemical equations 2.4 and 2.5 describe the gassing reactions that occur at the negative and positive electrodes respectively. The overall overcharge reaction is shown in equation 2.6 .

$$
\begin{aligned}
& 2 \mathrm{H}^{+}+2 \mathrm{e} \rightarrow \mathrm{H}_{2} \\
& \mathrm{H}_{2} \mathrm{O}-2 \mathrm{e} \rightarrow \frac{1}{2} \mathrm{O}_{2}+2 \mathrm{H}^{+} \\
& \mathrm{H}_{2} \mathrm{O} \rightarrow \mathrm{H}_{2}+\frac{1}{2} \mathrm{O}_{2}
\end{aligned}
$$

In a sealed lead-acid battery the recombination process allows most of the oxygen produced during overcharge to be recombined within the cell. This recombination process takes place at the negative electrode where oxygen in the presence of sulphuric acid diffuses to the lead surface (equation 2.7).

$$
\mathrm{Pb}+\mathrm{HSO}_{4}^{-}+\mathrm{H}^{+}+\frac{1}{2} \mathrm{O}_{2} \rightleftharpoons \mathrm{PbSO}_{4}+\mathrm{H}_{2} \mathrm{O}
$$

This process occurs in an unsealed lead-acid cell but at a rate that is too slow to prevent most of the hydrogen and oxygen gasses from escaping. In sealed cells the plates and separators are designed in such a way to allow the gas transfer between the plates which encourages the recombination reaction to occur. This combined with the fact that the cell is sealed and hence the gasses are not allowed to escape, allows the cell to be safely overcharged at recommended rates without loss of water. If overcharge does occur at 
higher than recommended rates the recombination process will not be able to keep up with the production of gasses and the cell will vent as the gas pressure builds up.

\subsection{Dryfit A212 Batteries}

The sealed lead-acid batteries used in the department's electric car are dryfit A212 12V batteries manufactured by Sonnenschein. An example of one is shown in figure 2.1. The technical specifications for this type of battery have been included in appendix D1.

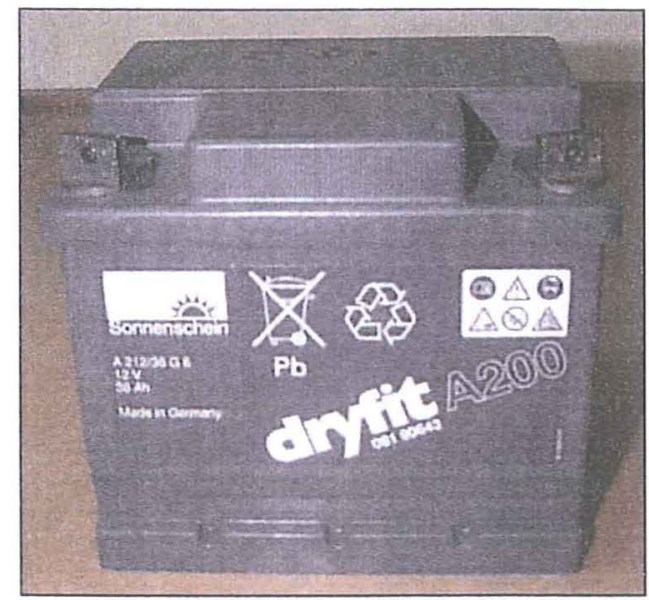

Figure 2.1: $\quad$ One of the batteries used in the electric car.

This type of battery is rated at 36Ah at a twenty hour discharge rate and weighs around $13 \mathrm{~kg}$. They use a gelled electrolyte and are constructed with grid type plates. The battery casing is made from polypropylene.

\subsection{Charging}

The process of charging a SLA battery involves replacing the energy that was removed from that battery during discharge. As with other battery technologies the charging process is not $100 \%$ efficient so it is necessary to replace more energy than was removed during discharging. SLA batteries do however have high charge acceptance ratios which are higher than traditional lead-acid batteries. The charge acceptance is typically very close to $100 \%$ until the battery approaches full charge at which point the charge acceptance falls as the overcharge reactions start to occur. Due to the use of an oxygen recombination process in SLA batteries it is important that SLA batteries are not overcharged at excessive charge currents to prevent the venting of gasses and the resulting loss of electrolyte.

There exist several methods for charging SLA batteries. These include constant voltage and constant current charge methods as well as charge methods incorporating both constant voltage and constant current. 


\subsubsection{Constant Voltage Charging}

Constant voltage charging is the simplest method of charging lead-acid batteries. It involves connecting a voltage source across the battery or string of batteries. The battery itself limits the charge current during the charge. As the battery charge state increases the charge current drops off until the battery is fully charged. At this point only a small residual current flows in the battery. This is shown by the typical charge characteristic in figure 2.2.

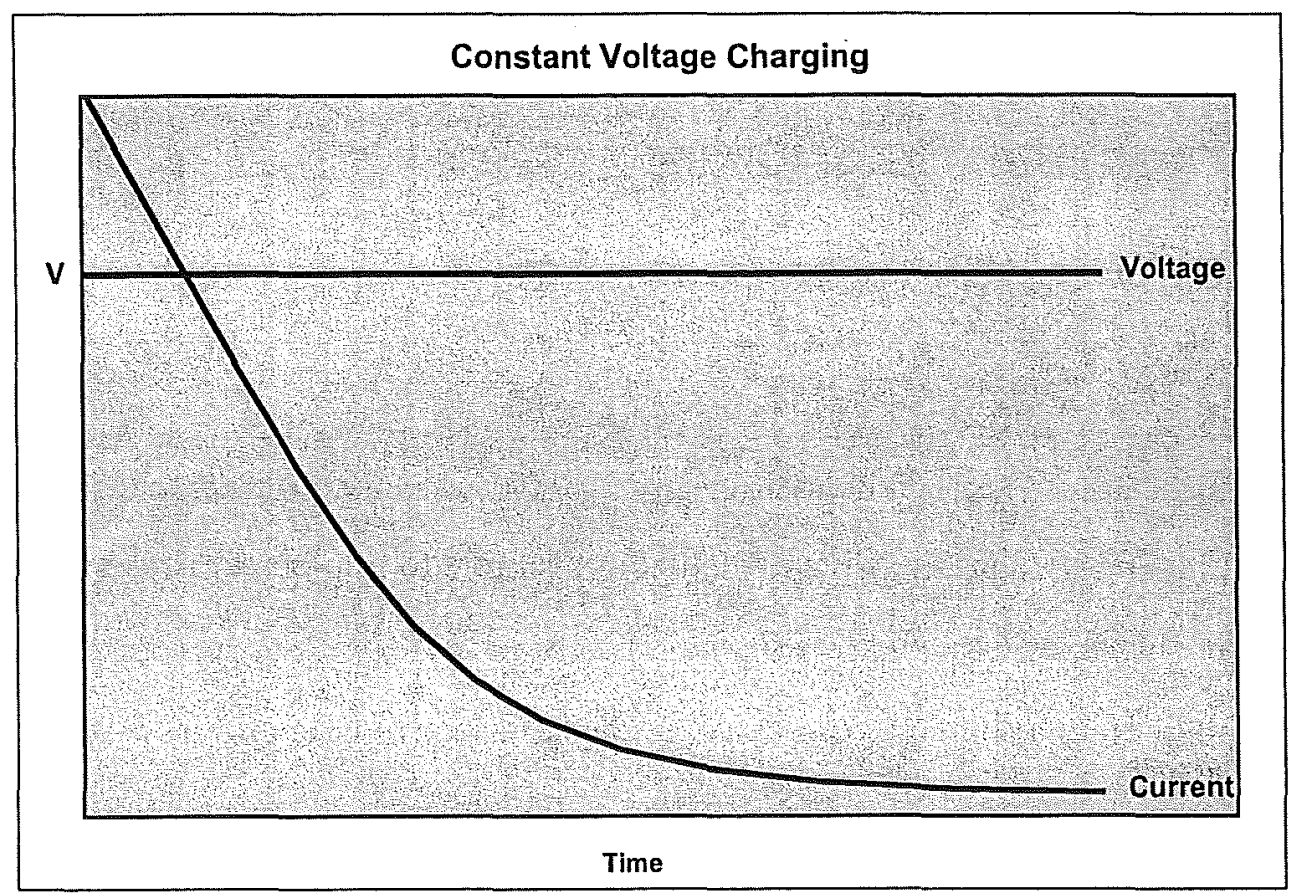

Figure 2.2: Constant voltage charging characteristic.

The voltage at which the battery should be charged depends on the temperature. At higher temperatures a lower charge voltage should be used. Conversely at lower temperatures a higher charge voltage is required. The charge efficiency reduces at lower temperatures so more energy needs be returned to the battery. The charge voltage also depends on the type of battery and whether it is used in cyclic applications or not. SLA batteries generally require higher charge voltages than conventional lead-acid batteries. Figure 2.3 shows charge voltages for the dryfit A200 series of batteries. Two ranges of permissible voltages are given for each temperature. The upper band is used when the battery is used in cyclic applications and the second range is used when the battery is in a standby application where it is left on a continuous float charge. 


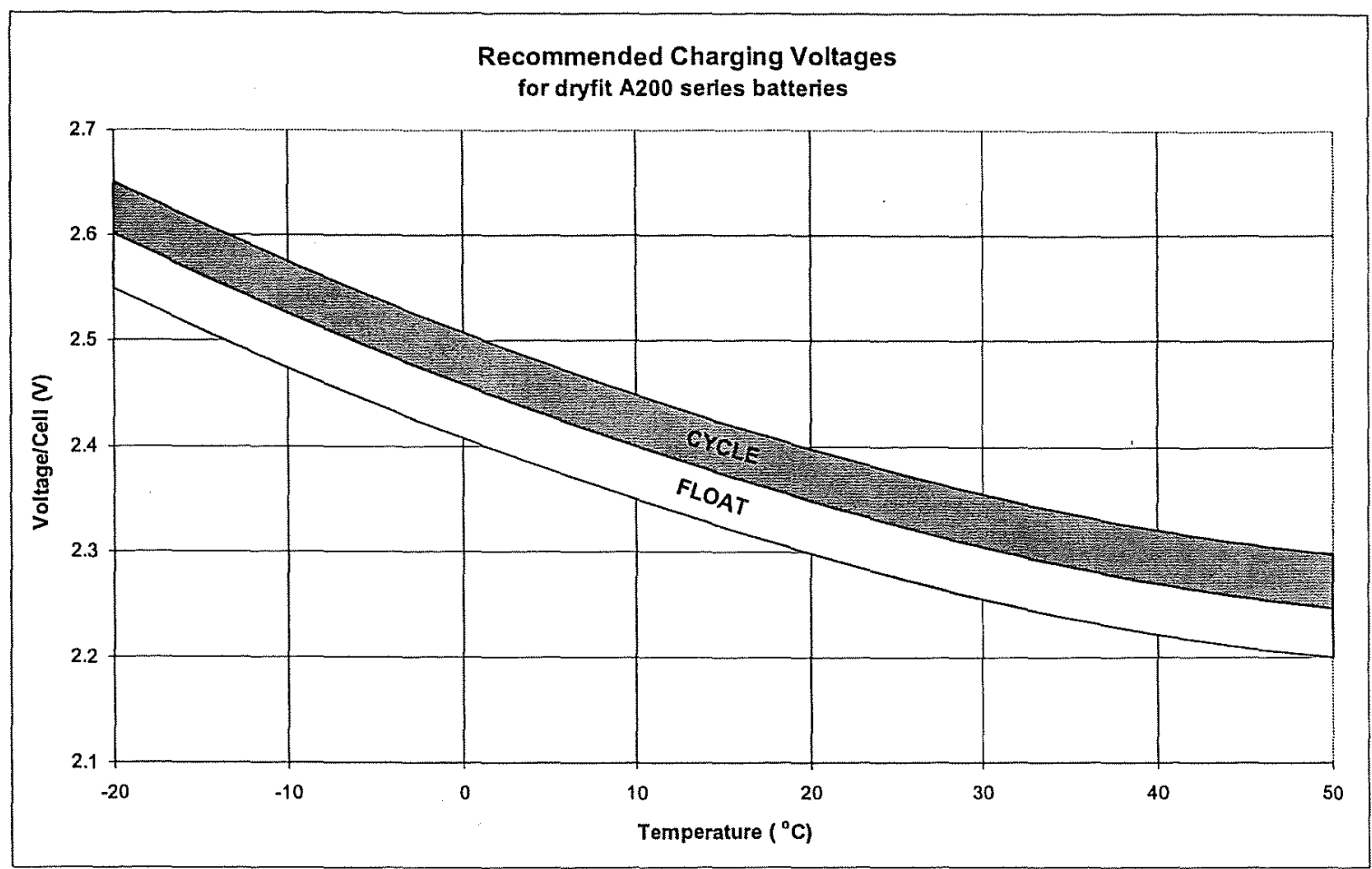

Figure 2.3: Recommended charging voltages for the dryfit A200 series batteries [6].

This charging method has the advantage that it is simple to implement as the battery itself takes care of the current regulation. It does however have the disadvantage that since the charge current falls off as the battery charges, the charge rate also slows. This means that it takes a long time for the last few percent of charge to be returned to the battery.

\subsubsection{Constant Current Charging}

Constant current charging is another charging method that may be used with lead-acid batteries. Figure 2.4 shows the voltage and current characteristics of this charging method. The battery voltage increases at a steady rate until the battery gets close to being fully charged. At this point the battery voltage rises rapidly until a peak is reached and begins to decrease again. After the peak in the voltage curve is reached the battery is well into overcharge. Since excessive overcharging should be avoided the charge current should be cut off just after the large increase in voltage is detected.

Since the charge current is fixed, the charge rate does not fall off as the state of charge of the battery increases as it does with constant voltage charging. This allows faster charge times to be achieved. This method does however require extra charger circuit complexity to detect the fully charged battery state so that overcharging does not occur. 


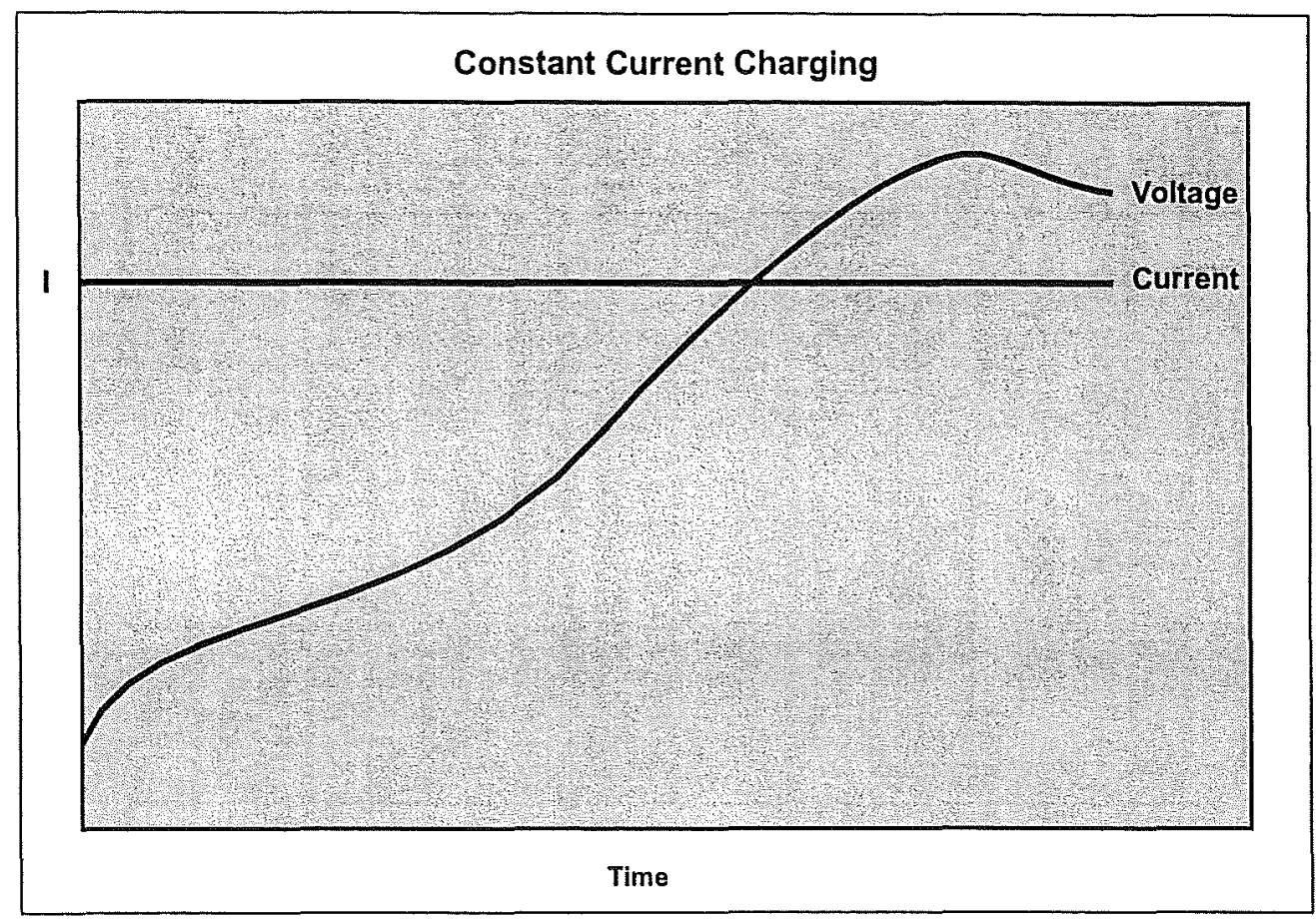

Figure 2.4: Constant current charge characteristic.

\subsubsection{Constant Current-Constant Voltage Charging}

A combination of the constant voltage and constant current charging methods can also be used which allows the benefits of both methods to be exploited. As the charge characteristic in figure 2.5 shows, a constant current charge is initially applied to the battery. When the recommended charging voltage of the battery is reached the charger switches over to a constant voltage charge and the battery is kept at this voltage until the charge is complete. This method is faster than the constant voltage charge method as the bulk of the charging is done at a constant rate. During the remainder of the charge the voltage is kept constant so overcharging does not occur. The detection of the change over point is relatively easy since all that is required is sensing of a voltage level.

If faster charge times are required this charge method can be modified to switching back to a constant current charge after a certain value of charge current is reached. This second charge current is at a significantly lower level than the initial charge current and is only maintained for a set length of time before the charger is switched off. 


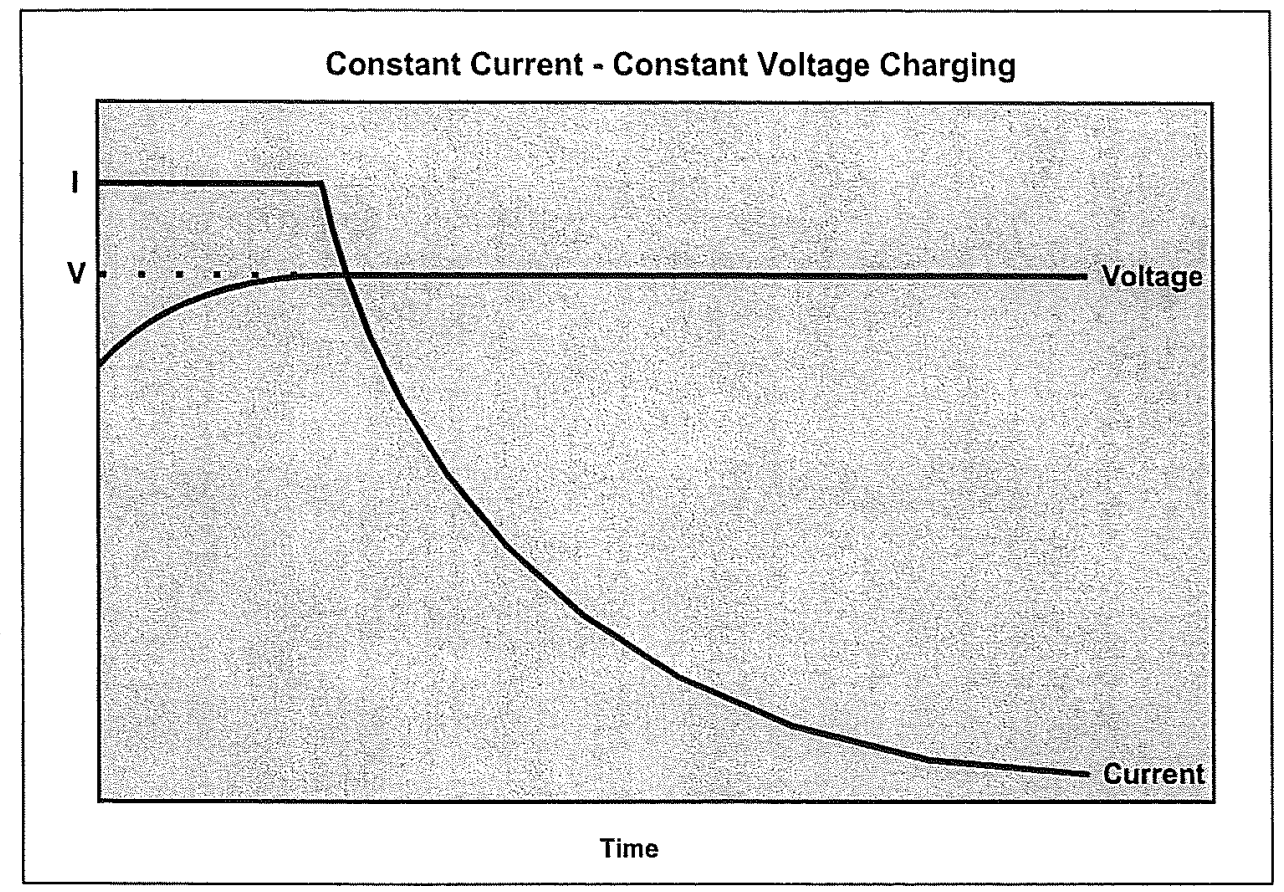

Figure 2.5: Constant current - constant voltage charge characteristic. 


\section{Sealed Lead-Acid Battery Characteristics}

\subsection{Discharge Characteristics}

Fully discharging a fully charged sealed lead-acid (SLA) battery at a constant current yields the discharge characteristics shown in figure 3.1. These characteristics were measured on a dryfit A212 battery rated at 36Ah using the battery data logging set up described in appendix $\mathrm{A}$.

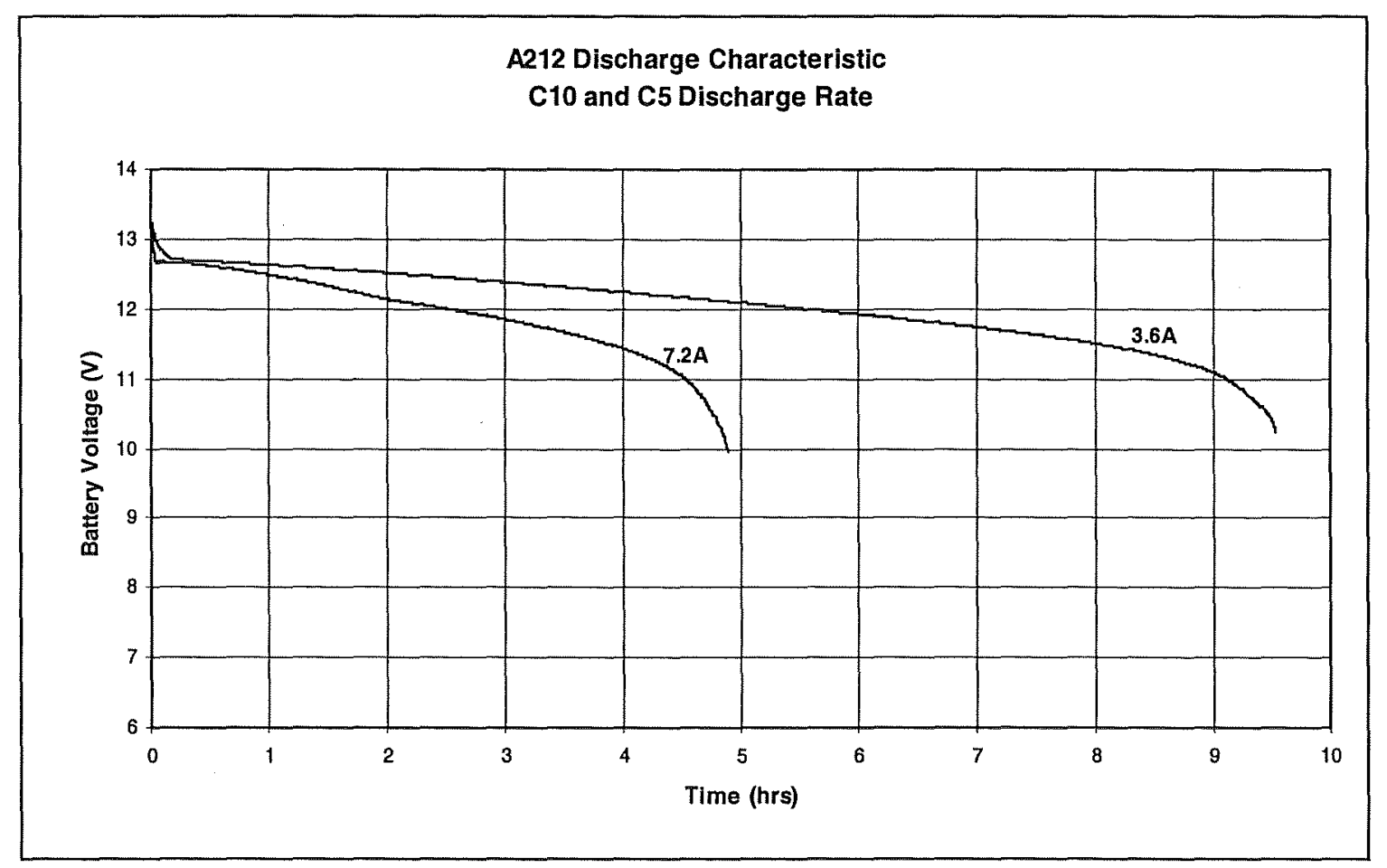

Figure 3.1: The discharge characteristic of a 36Ah A212 dryfit battery at constant discharge rates of $3.6 \mathrm{~A}\left(\mathrm{C}_{10}\right)$ and $7.2 \mathrm{~A}\left(\mathrm{C}_{5}\right)$.

At the start of the discharge the battery is fully charged hence the terminal voltage of the battery is higher than its nominal voltage. The terminal voltage quickly drops to a lower voltage and continues to slowly decrease as the battery is discharged. This decrease in voltage occurs due to the increasing internal resistance and the decreasing concentration of acid in the electrolyte.

As the battery gets towards the end of the discharge cycle the voltage begins to fall off more rapidly. The point where the discharge curve goes through the knee in the curve is 
considered the cut-off point since discharging the battery any further does not yield significantly more energy from it and could damage it.

At low discharge currents the discharge curve is almost flat. At high discharge currents the slope of the discharge curve becomes steeper as the terminal voltage of the battery drops more quickly due to the higher voltage drops in the battery. The final discharge voltage that the battery may be allowed to fall to before it enters deep discharge and possible damage also decreases at higher discharge currents due to the higher internal voltage drops.

\subsubsection{Self Discharge}

When a battery is left in an open circuit state it will gradually lose its stored capacity. This is known as self discharge. Gelled SLA batteries tend to lose very little of their capacity during storage compared to conventional wet celled lead-acid batteries due to the higher stability of the gelled electrolyte. The rate of self discharge depends on the construction and temperature of the battery. At higher temperatures self discharge occurs more rapidly. The dryfit A200 series of batteries are specified at having a self discharge rate of less than $0.1 \%$ per day at room temperatures.

\subsection{Battery Capacity}

The total useable capacity from a battery is the amount of capacity delivered between the fully charged and fully discharged states of the battery. There are many factors which influence the performance, and hence the total capacity that a battery can deliver. These include the discharge current, the type of discharge and the temperature of the battery. Additionally the capacity that a battery can deliver reduces as the battery ages until it reaches the end of its life.

Due to the number of factors influencing the battery capacity, batteries are rated under standard discharge conditions. Generally battery capacity is rated at either the ten $\left(\mathrm{C}_{10}\right)$ or twenty hour discharge rates $\left(C_{20}\right)$. This gives the total capacity than can be delivered over either a ten or twenty hour period at a discharge current of one tenth $\left(\mathrm{I}_{10}\right)$ or one twentieth $\left(\mathrm{I}_{20}\right)$ of the total rating.

The batteries used in the department's electric car have a capacity rating of $36 \mathrm{Ah}$ at the twenty hour discharge rate. This means that they can deliver $36 \mathrm{Ah}$ over a twenty hour period at a discharge current of $1.8 \mathrm{~A}$.

\subsubsection{Discharge Current}

The total capacity of a battery is significantly influenced by the rate at which current is removed from it. At higher discharge currents the battery is able to deliver less capacity 
than at low discharge currents. This is because the capacity is limited by the diffusion rate of the electrolyte and also because the internal resistance of the battery causes a larger voltage drop at higher discharge currents and hence results in higher IR losses.

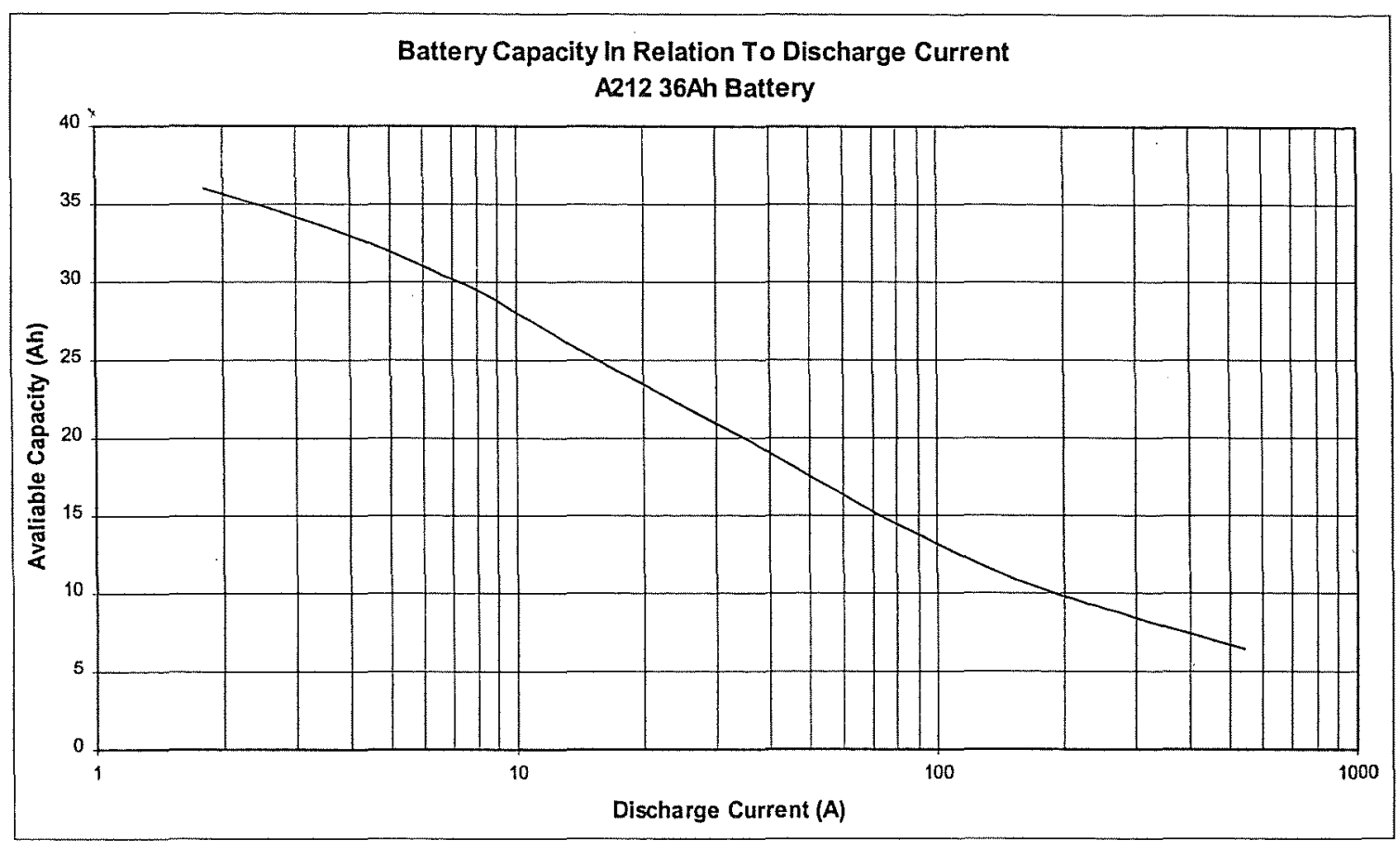

Figure 3.2: $\quad$ The capacity of a 36Ah A212 dryfit battery in relation to the discharge current [6].

Figure 3.2 shows the relationship between discharge current and available capacity for the A212 dryfit sealed lead-acid battery used in the department's electric car. This relationship can be approximated by the Peukert equation 3.1 [7].

$$
C_{t}=k \cdot I^{n}
$$

In this equation $\mathrm{k}$ and $\mathrm{n}$ are constants which are experimentally determined for each particular battery type. For the A212 dryfit batteries $\mathrm{k}$ was found to be 41 and $\mathrm{n}$ to be -0.17 .

\subsubsection{Type of Discharge}

The type of discharge also influences battery capacity. If during the discharge the battery is allowed to rest it will regain some of its capacity. This occurs since the diffusion rate of the bulk electrolyte is slower than the local diffusion that occurs around the plates of the battery. During a period of rest the diffusion of the bulk electrolyte compensates for the local diffusion around the plates and hence the terminal voltage rises.

The amount of capacity regained is more significant at higher discharge currents and is also influenced by the battery temperature and the length of the rest period. 


\subsubsection{Temperature}

Temperature influences battery capacity due to changes in the resistivity and viscosity of the electrolyte. At lower temperatures the reduction in chemical activity and the increased internal resistance of the battery reduces the total battery capacity. The reduction in capacity at low temperatures is also influenced by the discharge current. Figure 3.3 shows the relationship between ambient temperature and capacity at several discharge currents for a dryfit SLA battery.

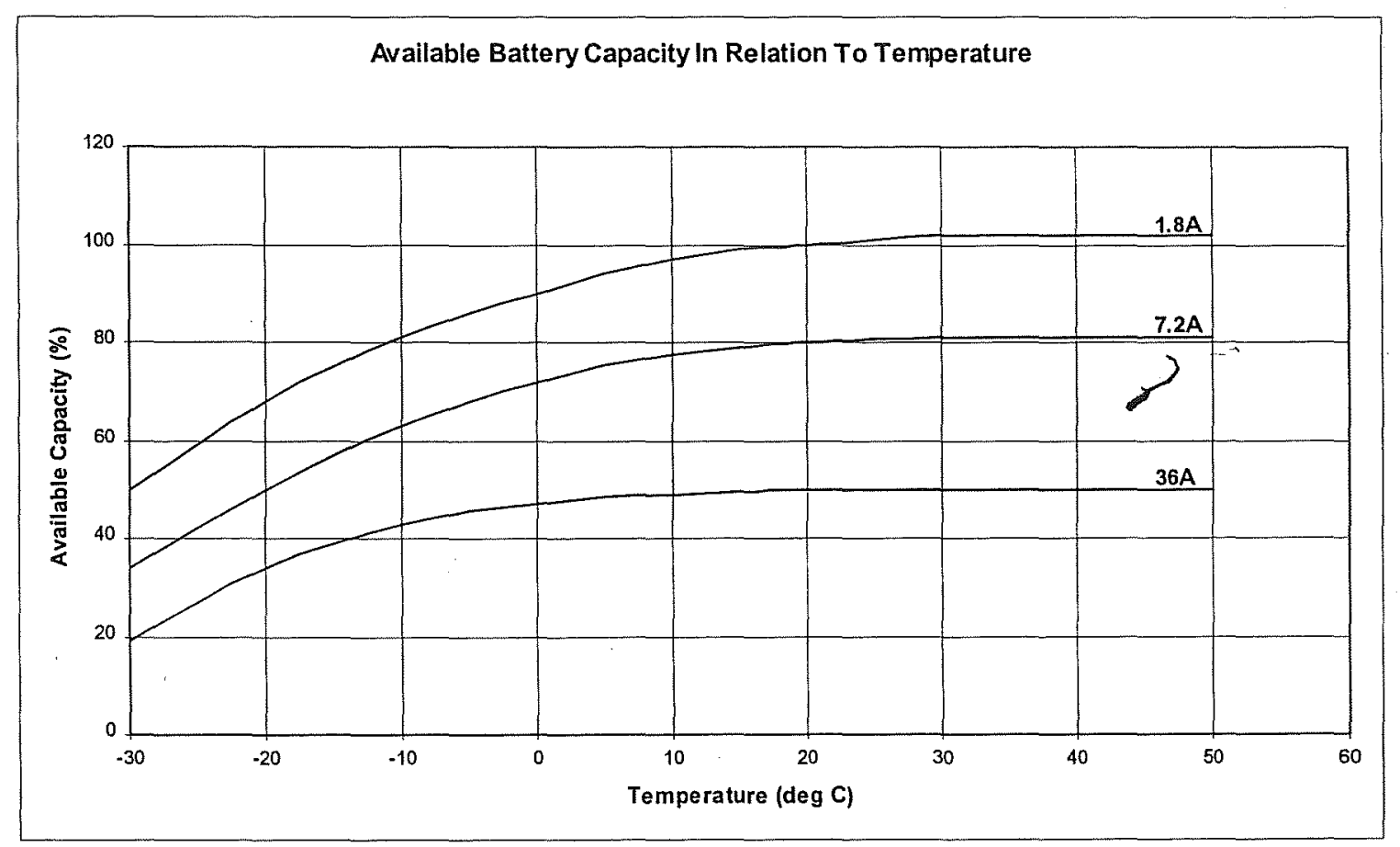

Figure 3.3: Battery capacity variation in accordance with temperature for a dryfit battery [6].

From figure 3.3 it can be seen that at low discharge currents the total capacity is more or less constant above $20^{\circ} \mathrm{C}$. The capacity falls off at lower temperatures until the electrolyte freezes at below around $-25^{\circ} \mathrm{C}$. At higher discharge currents the range over which the capacity is close to constant extends down to lower temperatures. This is due to the internal self heating that occurs in the battery at higher discharge currents.

\subsection{Open Circuit Voltage Characteristics}

Under no-load conditions, the open circuit voltage across the terminals of a battery can be measured. Under these conditions there are no voltage drops present in the battery as no current is following through the battery. The open circuit voltage is therefore very close to the theoretical terminal voltage of the battery which is a function of the type of electrodes and chemical properties of the battery. 


\subsubsection{Open Circuit Voltage Relation to State of Charge}

The concentration of acid in a lead-acid battery relates directly to the battery's state of charge. In a wet celled battery specific gravity measurements performed on the electrolyte give the concentration of acid in the electrolyte, and therefore can be used to indicate the battery state of charge. The open circuit voltage is also a function of the concentration of acid in the electrolyte. A good linear relationship exists between the open circuit voltage of the battery and its state of charge. This relationship is shown in figure 3.4 for a dryfit $\mathrm{A} 212$ battery.

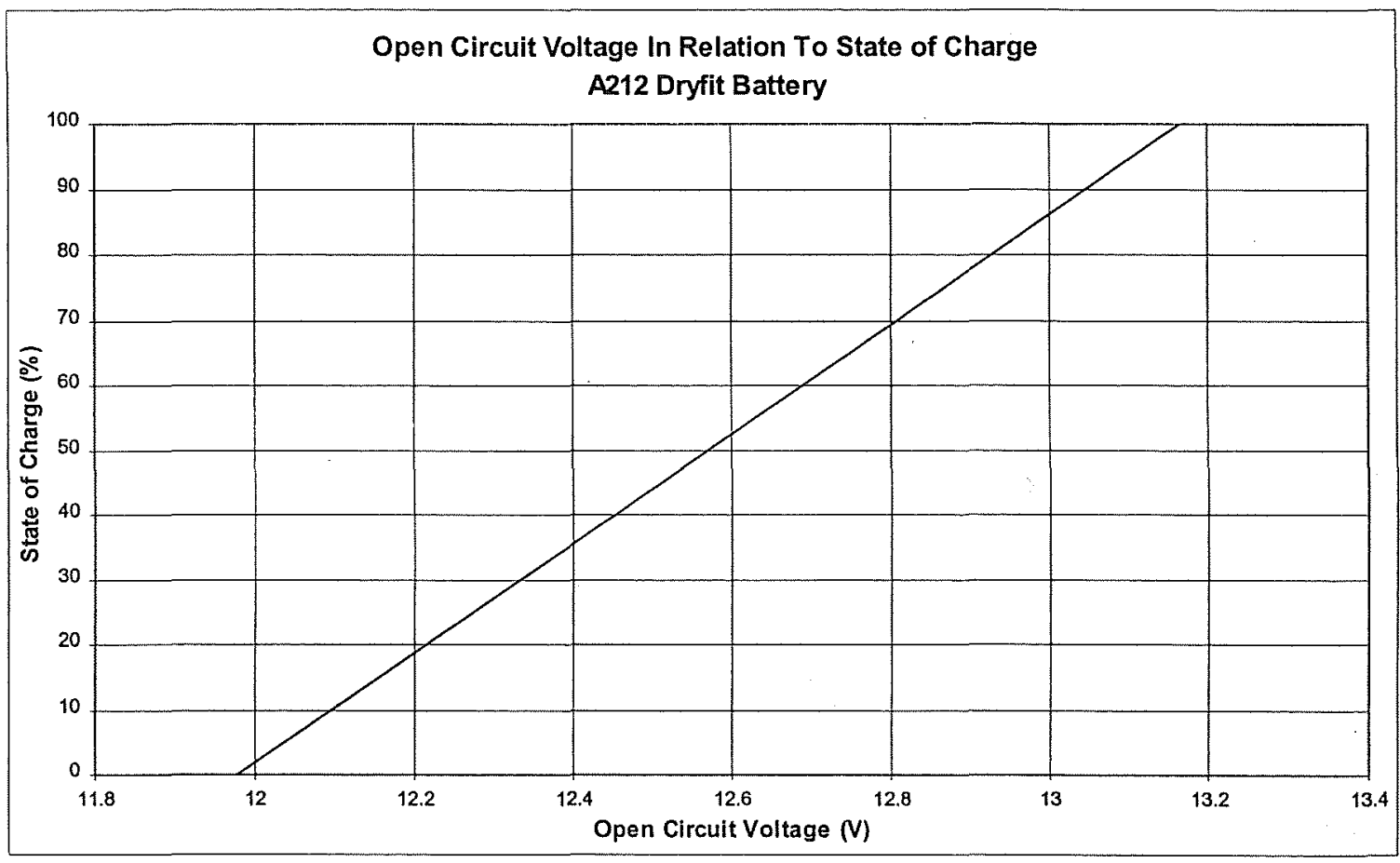

Figure 3.4: Relationship between open circuit voltage and state of charge for an A212 dryfit battery.

The relationship between open circuit voltage and state of charge has little dependency on the temperature and past history of the battery [8]. Different lead-acid batteries however do have different ranges of open circuit voltage, so the relationship is therefore different for different types of batteries.

\subsubsection{Open Circuit Voltage Recovery Characteristics}

A reliable open circuit voltage measurement cannot be made on a battery until it has fully stabilised. The time taken for the battery to stabilise after a discharge depends on the type of battery, and can range from half an hour to several hours. Batteries with gelled electrolytes typically take longer to stabilise than wet celled batteries. Experimental results have shown that the A2,12 dryfit batteries typically take up to four to five hours to fully stabilise. 


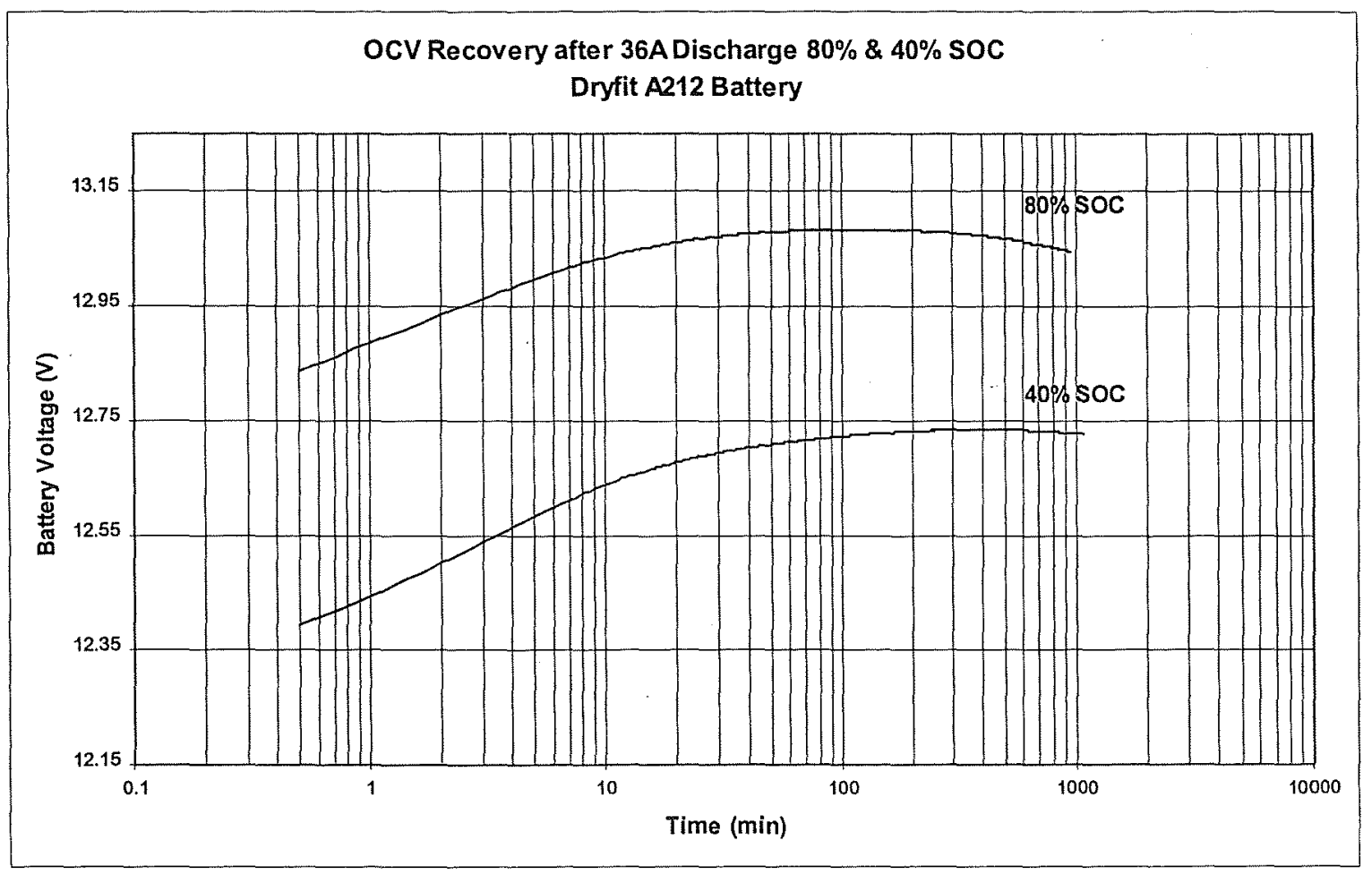

Figure 3.5: Open circuit voltage recovery curves measured on an A212 dryfit battery after discharges to $80 \%$ and $40 \%$ of remaining capacity at $36 \mathrm{~A}$.

Figure 3.5 shows typical open circuit voltage recovery characteristics on a semi-log scale. These curves were measured after the battery had been discharged so that it had $80 \%$ and $40 \%$ of its capacity remaining. As figure 3.5 shows, after the load is disconnected from the battery the terminal voltage initially rises rapidly. The rate of this rise tappers off until the battery reaches a stable open circuit voltage. After this point the terminal voltage begins to fall due to internal self discharge of the battery.

\subsection{Life Expectancy}

Batteries have a limited service life which is either expressed in years for standby applications or number of discharge cycles that can be delivered in applications where the batteries are continuously cycled.

Over the cycle life of a SLA battery the capacity actually increases over the first few discharge cycles until the capacity reaches a peak and then begins to gradually fall off as the battery ages. A battery is usually considered to have reached the end of its useful life when it can deliver less than $80 \%$ of the capacity it could when it was new [9].

In standby applications where the battery spends most of its life being float charged, a typical battery can be expected last for up to eight years. The service life reduces as the operating temperature of the batteries increases. This is caused by the increase in the rate of corrosion that occurs inside the batteries at high temperatures. 


\subsubsection{Depth of Discharge}

In cyclic applications the depth of discharge (DOD) has a significant influence on the cycle life of the batteries. Figure 3.6 shows the relationship between depth of discharge and the number of cycles for a typical SLA battery. As can be seen a battery that is subjected to a $100 \%$ DOD will last for fewer cycles than a battery that is always discharged to $50 \%$ DOD. The number of cycles that a battery can deliver depends on the type and construction of the battery. Discharging SLA batteries to greater than $100 \%$ DOD will significantly shorten their life and can impair their ability to accept charge.

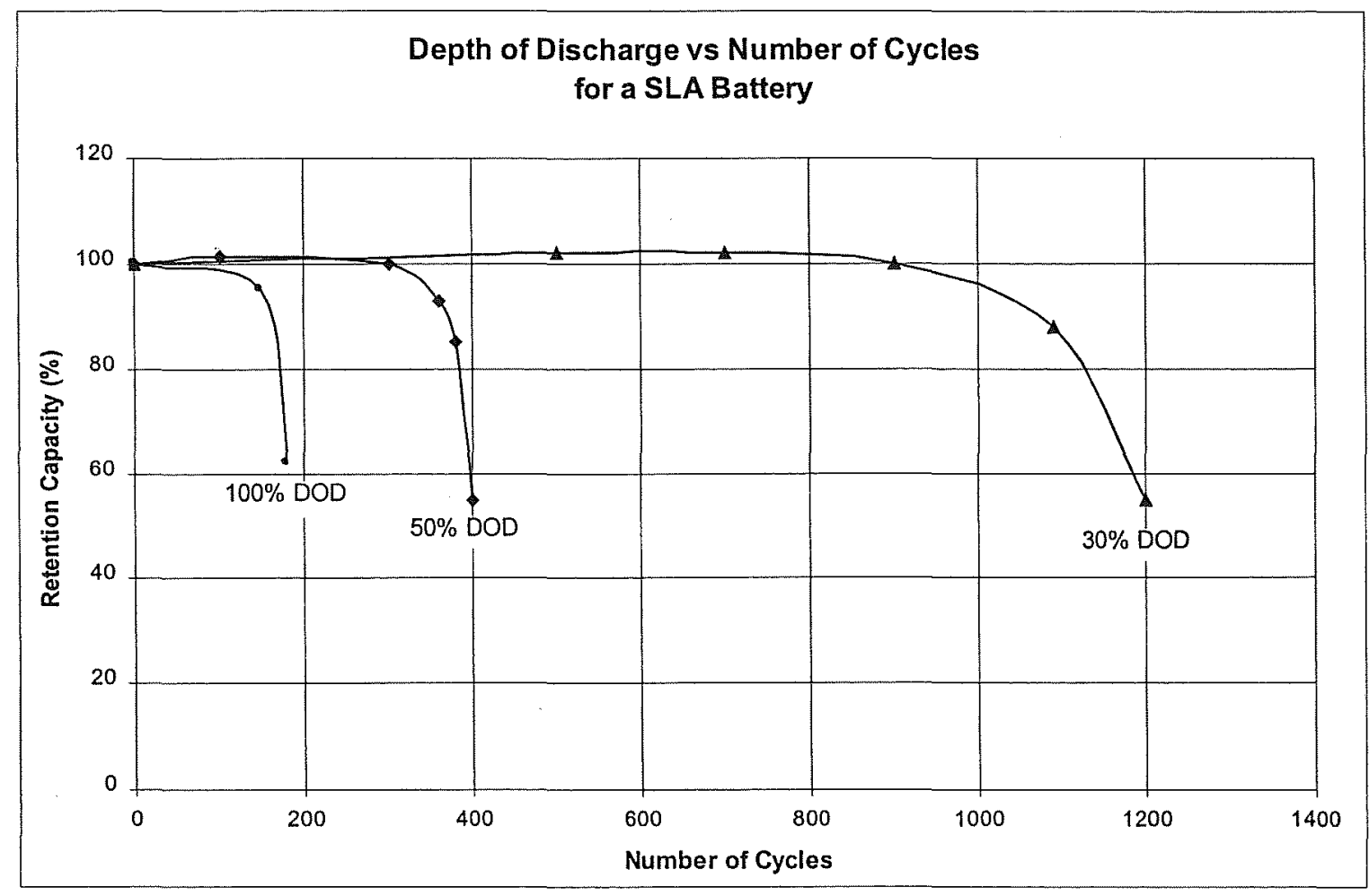

Figure 3.6: The relationship between DOD and cycle life for a typical sealed leadacid battery [10].

The dryfit A200 series of batteries have a slightly better cycle life than the battery shown in figure 3.6. They have a specified cycle endurance of 400 cycles at $60 \%$ DOD which falls to only 200 cycles at $100 \%$ DOD.

In most applications a compromise is made between the depth of discharge and cycle life to gain as much capacity from the batteries in each cycle while gaining good cycle endurance from them. To achieve this the maximum depth of discharge is usually set at $80 \%$. One way of improving cycle endurance is to oversize the batteries for the application so that the depth of discharge is reduced. This however also adds to the weight of the battery bank, which may not be desirable in applications where weight is a consideration such as in electric vehicles. 


\subsubsection{Charging}

The type of charging method used to recharge SLA batteries also has an impact on their life. If the battery is charged at a voltage that is too high then production of oxygen in the battery will occur at a rate higher than the oxygen recombination process can handle. If this occurs oxygen may be vented rather than being returned to the electrolyte resulting in electrolyte loss. Also under overcharge conditions temperatures will increase inside the battery due to the fact that the charge current is only being used in the overcharge reactions rather than charging the battery. At high temperatures the reaction rates inside the cells also increase helping to further decrease the life of the battery. 


\section{Battery Monitoring Techniques}

In applications where secondary batteries are used as a power source it can be desirable to know the state of charge and the health of the batteries. State of charge information is useful as it takes the guesswork out of knowing how much longer the batteries will last during discharge and tells the user when recharging is necessary. Knowing the state of charge also helps prevent the batteries from being inadvertently over discharged.

Information on the health of batteries is also useful as it helps avoid failures due to premature battery failure and can be used to tell precisely when battery replacement is necessary. Knowing this type of information is particularly important in standby power supplies and UPS applications where the batteries must be relied on to provide power when the main supply fails.

Traditionally in large battery banks the condition of each battery is assessed manually by periodically measuring the voltage and the specific gravity of each battery in the bank. Battery inspections of this type are both labour intensive and risky to both personnel and the battery itself [11]. As a result automated monitoring systems are becoming increasingly popular.

The main requirements for battery monitoring systems vary depending on the application. In standby or UPS systems the batteries are continuously float charged while the main supply is present. They are only discharged when the main supply fails. It is therefore important to know the state of health of the batteries and from this information detect potential battery failures. State of charge information is less important since the batteries are kept in a fully charged state by the charger when the main supply is present. It is only during failure of the main supply where the batteries are being discharged that this information is useful to determine the remaining battery runtime.

In cyclic applications the battery state of charge is an important battery parameter since the batteries are continuously put through discharge and charge cycles. State of charge information is particularly important in traction applications such as electric vehicles as knowing the state of charge helps avoid vehicle strandings while insuring that the best use is made of the available battery capacity. State of health information is useful in determining when the battery bank has reached the end of its useful life and requires replacement.

There have been many methods proposed for measuring the state of charge of batteries, each with varying degrees of accuracy. Measuring state of health is a more difficult task. 
This chapter considers some of these measuring methods and their suitability for use in an electric vehicle application. Although some of the methods examined will also work for other battery technologies, methods for monitoring lead-acid batteries are considered since lead-acid batteries are currently the most common battery type used in electric vehicles and are the type used in the Department's electric car.

\subsection{Measuring State of Charge}

The state of charge of a battery is usually expressed as a percentage of the total battery capacity and can be calculated by equation 4.1 , where $C_{t}$ is the total capacity of the battery and $C_{r}$ is the capacity remaining in the battery.

$$
S O C=\frac{C_{r}}{C_{t}} \times 100 \%
$$

Battery capacity can be measured in watt-hours but is more commonly measured in ampere-hours since the voltage from a battery is close to constant over most of its discharge.

Since $C_{r}$ in equation 4.1 is difficult to define and even more difficult to measure, the used capacity $C_{u}$ is more useful when calculating the state of charge as in equation 4.2.

$$
S O C=\frac{1-C_{u}}{C_{t}} \times 100 \%
$$

$C_{u}$ if measured in ampere-hours is the integral of current over time as shown by equation 4.3 where $I$ is the current drawn from the battery in amperes and $t$ is the time since the battery was fully charged in hours.

$$
C_{u}=\int_{0}^{t} I . d t
$$

The remaining parameter required is the total battery capacity $C_{t}$. This parameter is the most difficult to determine and is influenced by many factors as described in section 3.2.

\subsubsection{Coulometric}

The equations above are used directly in the coulometric state of charge (SOC) measuring technique. This method basically counts the number of ampere-hours removed from the battery or replaced while the battery is being discharged or charged. This is achieved by measuring the battery current and using equation 4.3 to calculate the used capacity. 
The coulometric measuring method in its simplest form assumes the total capacity of the battery is fixed and does not vary with the discharge current, temperature or age of the battery. This assumption can result in an accumulation of error in the measurements over a period of time if the operating conditions and hence the assumed total capacity varies.

The accuracy of the coulometric technique can be improved during discharge by using the Peukert equation (equation 3.1) to model the relation between total battery capacity and the discharge current. This requires that the total battery capacity is recalculated over each measurement period and used in the state of charge calculation. If the temperature characteristics of the battery being monitored are known, this information can also be used to compensate the total battery capacity for temperature variations.

During charging coulometric measurements must take into account the charge efficiency of the battery. The charge acceptance in sealed lead-acid batteries is high and hence the charge efficiency can be assumed to be $100 \%$ until the battery comes close to gassing. The charge efficiency then drops to around $85 \%$ until the battery is fully recharged. To account for state of charge errors during charging, the fully charged point can be detected and used to force the state of charge to $100 \%$.

Battery recuperation effects can also be compensated for by taking the average discharge current up to the present time and using this value to calculate the total capacity from the Peukert equation (3.1). The average current can be calculated using equation 4.4 where $C_{\mathcal{U}}$ is the capacity used up to the present time and $t$ is the time since the discharge began. During periods of rest $C_{u}$ remains constant while $\bar{I}$ decreases as the total time increases. This means that the calculation of the total capacity from equation 4.4 will increase allowing for recuperation during the rest period.

$$
\bar{I}=\frac{C_{u}}{t}
$$

Coulometric measurements provide a reasonably accurate short-term indication of the state of charge if the variation in capacity with discharge current is taken into account and can be made to account for recuperation periods and temperature effects. Since coulometric measurements are based on fixed battery models however this SOC measuring method cannot account for battery ageing. This limitation will therefore lead to inaccuracies as the battery being monitored ages.

\subsubsection{Specific Gravity}

Measuring the specific gravity of the battery's electrolyte is one of the more common conventional methods of obtaining the state of charge of a lead-acid battery. The specific gravity is usually measured by manually drawing some of the electrolyte into a hydrometer, although this can also be performed by using electronic sensors inside the 
battery itself. The specific gravity of the electrolyte shows the concentration of acid in the electrolyte which correlates directly to the battery's state of charge. The specific gravity does however need to stabilise after the battery is discharged. This stabilisation period can take typically from half an hour to many hours, so measurements cannot be taken continuously while the battery is being discharged. This method also makes it necessary to gain access to the electrolyte in the battery which is somewhat difficult with sealed batteries unless sensors are built into the batteries. These factors tend to limit the usefulness of this method for automated measuring of the SOC in this type of application.

\subsubsection{Open Circuit Voltage Measurements}

As described in section 3.1 the open circuit voltage of a battery, like the specific gravity is also a function of the concentration of acid at the plates. Since a good linear relationship exists between the open circuit voltage of the battery and its state of charge (figure 3.4), the open circuit voltage can be used to calculate the SOC. The long stabilisation time required before a reliable open circuit voltage measurement can be made and the fact that the load must be disconnected however limit the usefulness of this method as an indicator of SOC.

\subsubsection{Loaded Voltage Measurements}

The battery voltage with a load present, like the open circuit voltage, has a close to linear relationship to the specific gravity and hence SOC if the load current is constant [8]. This is demonstrated by the close to linear discharge profiles in figure 3.1. In most devices, including electric vehicles, the load current is not fixed and varies over a large range. The loaded voltage of the battery also varies as the load varies. It is therefore difficult to use this method to indicate SOC under these conditions.

\subsubsection{Open Circuit Voltage Prediction}

The problem associated with the long stabilisation period of the open circuit voltage can be overcome by making a prediction of the open circuit voltage a short time after the load has been disconnected from the battery [8].

The open circuit voltage recovery characteristic was described in section 3.3.2. This recovery curve can be approximated by two asymptotes as shown in figure 4.1 . 


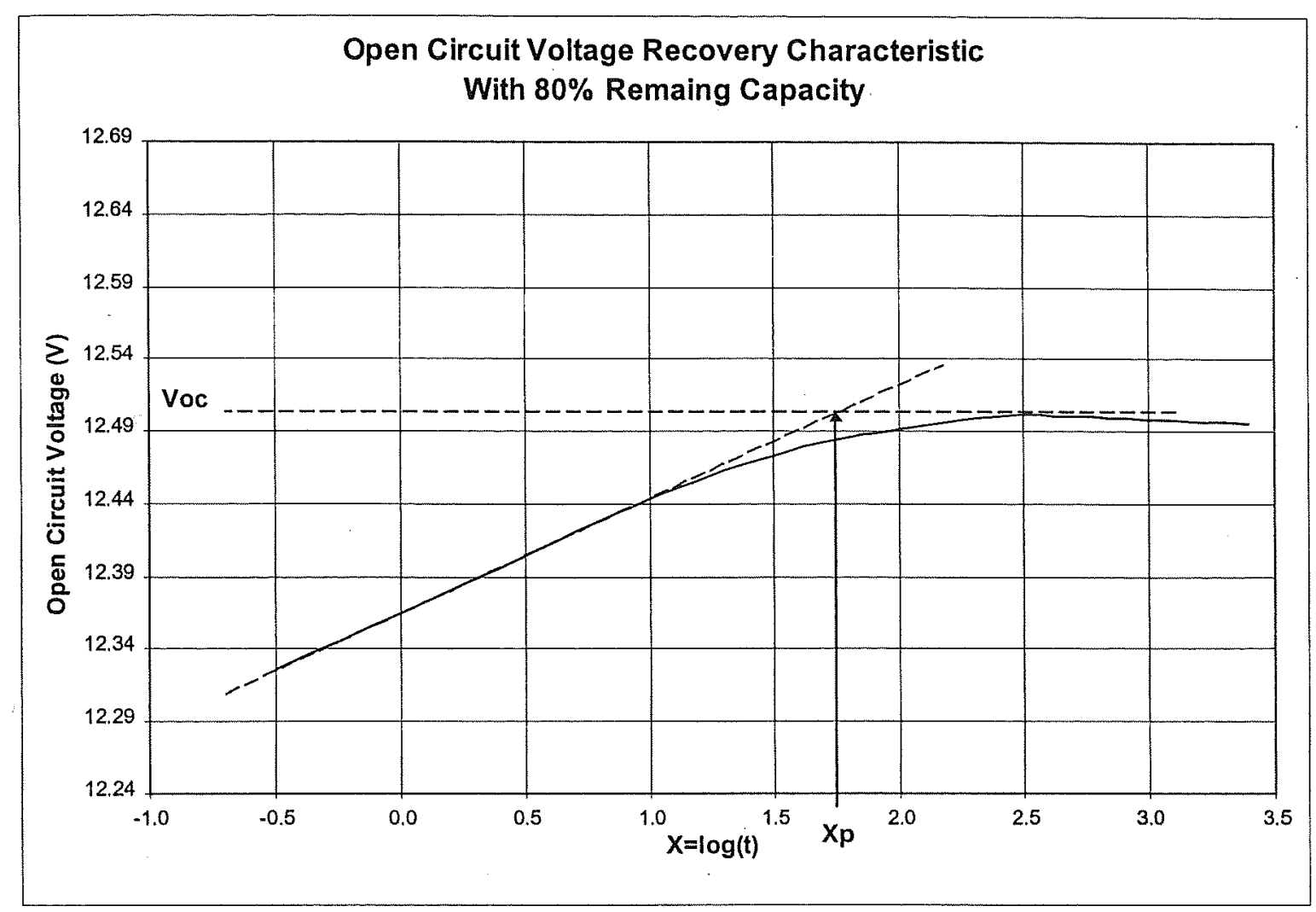

Figure 4.1: Approximation of the open circuit recovery characteristic by two asymptotes and the derivation of $X_{p}$.

In order to obtain a linear relationship the recovery curve in figure 4.1 has been plotted against $\log t$. The variable $X$ is used which is related to time $t$ by equation 4.5.

$$
t=10^{X}(\min )
$$

The value of $X$ at which the two asymptotes cross $\left(X_{p}\right)$ can be found. $X_{p}$ has experimentally been found to be reasonably consistent with both the type of battery and the state of charge [8], although batteries with gelled electrolytes tend to have larger values of $X_{p}$ since they typically take longer to reach this point.

Once $X_{p}$ is known, the slope of the open circuit voltage recovery curve can be used to predict the fully stabilised open circuit voltage. Equation 4.6 shows how this is done where $M$ is the slope of the first asymptote and $V_{O}$ is the battery voltage straight after the load is disconnected.

$$
V_{o c}=M \cdot X_{p}+V_{o}
$$

The slope $M$ is calculated by taking two measurements of the battery voltage a short time after the load has been disconnected. If the first measurement is made after one minute ( $X$ $=0)$ and the second measurement after $t_{2}$ minutes $\left(X=X_{2}\right)$ then $M$ can be calculated using equation 4.7 . 


$$
M=\frac{V_{o c 2}-V_{o c 1}}{X_{2}}
$$

The predicted open circuit voltage calculated from equation 4.6 can then be used to determine the state of charge using the open circuit voltage to state of charge relationship (figure 3.4). This is shown in equation 4.8 where $M_{S O C}$ and $Y_{O S O C}$ are the slope and $\mathrm{Y}$ intercept of the line in figure 3.4 .

$$
S O C_{o c v}=M_{s o c} \cdot V_{o c}+Y_{o s o c}
$$

The open circuit voltage prediction cannot be used after a charge cycle since the recovery characteristic after charging is different to the recovery after discharge as shown by figure 4.2. The recovery after discharge will also be influenced by the previous charge cycle until the battery has been discharged significantly. As a result the open circuit voltage prediction cannot be relied on until the battery's state of charge falls to $80 \%$ or below.

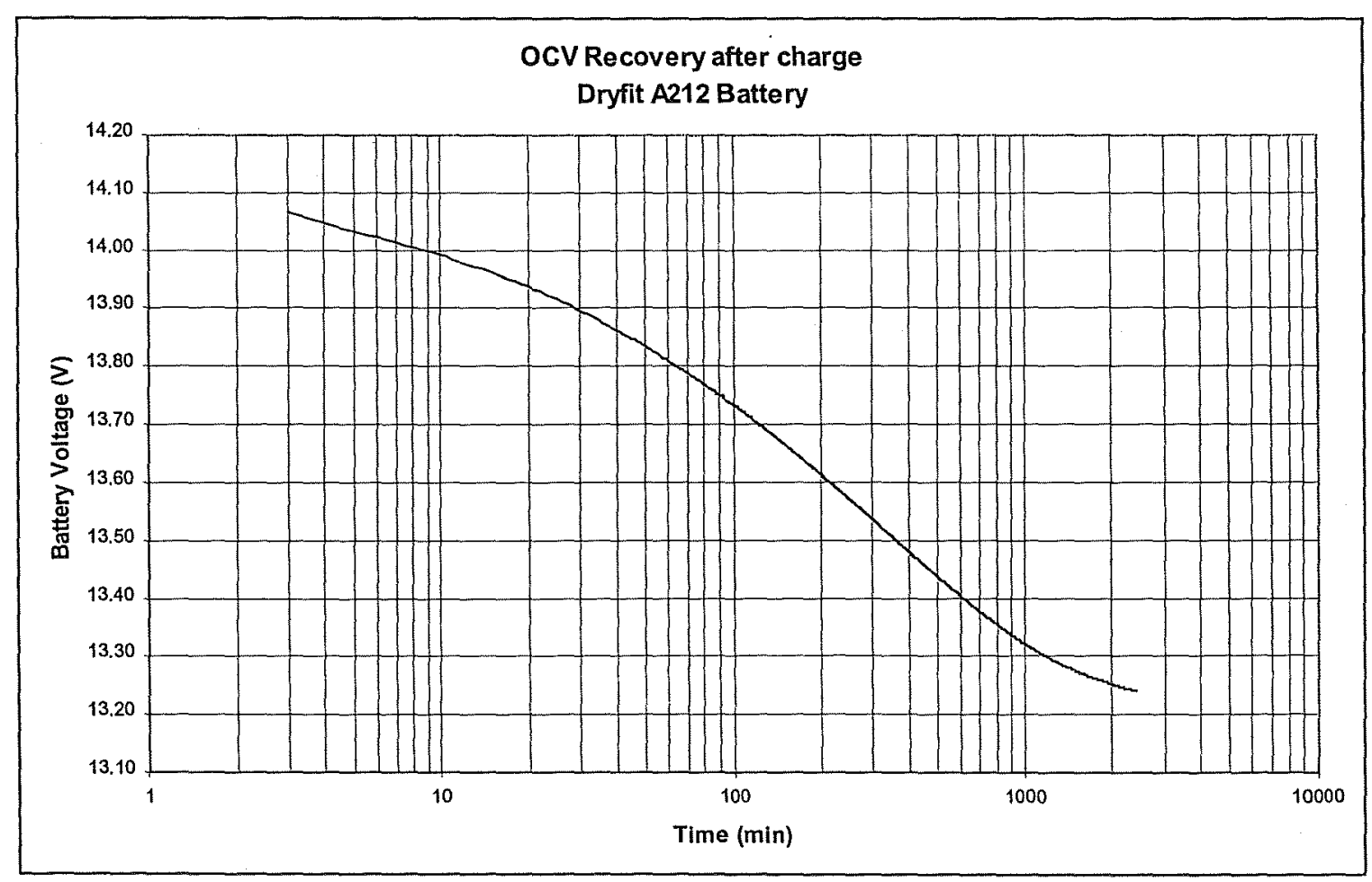

Figure 4.2: Open circuit recovery characteristic after a charge cycle.

This method of predicting the open circuit voltage provides an accurate estimate of the state of charge of a battery without the need to wait for the battery to fully stabilise. Since the state of charge is based on the open circuit voltage which is in turn determined by the chemical state of the battery this method gives a state of charge estimate which closely relates to the battery's actual state. This means that this method will still give reliable results as the battery ages since it is based on actual battery measurements rather than 
battery models. The battery must still be disconnected from the load however, in order to perform the open circuit voltage measurements, so it can not provide a continuous indication of the state of charge during discharge.

\subsection{Adaptive State of Charge Measurement}

Of the methods discussed above no single method meets all of the requirements desirable for a state of charge measurement system in a cyclic application. The coulometric method comes closest as it gives a continuous estimate of the state of charge during both discharge and charge. This method does however have several shortcomings. It cannot account for changes in capacity as the battery ages and it is also prone to accumulate errors over time. If this method is used these shortcomings must be overcome by using some means of correcting these errors.

The open circuit voltage prediction method cannot give state of charge during discharge but it can, when the batteries are disconnected from the load, provide a more accurate estimate of the state of charge. By comparing this estimate to the last value obtained from the coulometric method an error correction factor can be generated which can be used to correct the coulometric measurements during the next discharge. As the battery ages the state of charge obtained from the open circuit voltage will be less than that estimated from the coulometric measurements and hence the error correction factor will allow the coulometric calculations to compensate for this.

\subsection{Measuring State of Health}

Predicting battery state of health is a more difficult task than measuring battery state of charge, and as a result, fewer methods have been proposed so far in the literature. One method that has been proposed [12] uses the battery internal impedance, battery voltage and information about the operating environment of the battery to make an estimate of the remaining life span of the battery. This method is however more suited to batteries used in standby applications rather than cyclic applications.

In section 3.4 it was shown that in cyclic applications the depth of discharge has a significant influence on the life of the battery (figure 3.6). If enough information is known about the relationship between the depth of discharge and the cyclic life then a reasonably accurate prediction can be made about the number of remaining cycles the batteries will deliver before replacement is required. If this information is not given by the battery manufacturer then this information must be obtained by experimentation. To do this would require cycling batteries of the same type through many discharge cycles at various depths of discharge. This would be a very time consuming process. The accuracy of this state of health predicting method would also depend not only on the accuracy of this data, but also on the reliability of this data under different battery operating conditions. 
The adaptive state of charge measuring method described in section 4.2 has inherent in it a way of indicating the state of health as well as the state of charge. This method uses the coulometric method to indicate state of charge while the car is running which is based on battery models that describe the performance of a healthy battery. These measurements are compensated by open circuit voltage based state of charge measurements that give a state of charge which is an indication of the actual state of the battery. The compensation factor obtained by comparing the two state of charge measuring methods can therefore be used to derive the state of health of the battery. With this method a healthy battery will have a state of charge derived from the two measuring methods which is very similar and hence the state of health will read near $100 \%$. As the battery ages the uncompensated coulometric measurement and the open circuit voltage based measurements will be different and the ratio of the two will give a good indication of the state of health. The accuracy of this value will depend on the accuracy of both the battery models used in the coulometric based measurements and the accuracy of the open circuit voltage prediction calculations.

\subsection{Battery Monitoring for Electric Vehicles}

In an electric vehicle a battery monitoring system must be able to calculate the state of charge to an accuracy that will prevent vehicle strandings from occurring while allowing the full range of the vehicle to be exploited. This typically requires an accuracy of $\pm 5 \%$ or better. In addition to this a continuous indication of the state of charge is required while the vehicle is operating. The state of health calculations do not however need to be updated as often and one update every time the vehicle is used should be sufficient.

The adaptive state of charge measuring method described above meets all of these requirements. It provides continuous state of charge measurements while the vehicle is running and makes corrections for errors and calculates the state of health a short time after the vehicle has been shutdown. In addition only non-invasive measurements of battery current, temperature and voltage are required to implement this method. For these reasons this method was chosen to implement in the Department's electric car. The next two chapters look at the development of both the hardware and software to perform battery monitoring using this method. 


\section{System Hardware}

\subsection{Requirements}

In order to perform battery monitoring using the methods outlined in chapter 4 , the battery monitor must take measurements of the battery current, temperature and terminal voltage. In order to measure the state of health of each of the batteries the terminal voltage across each of the twenty batteries must be measured. The battery current only needs to be measured at one point since the batteries are all connected in series. Since the batteries are divided into three groups it is sufficient to measure the temperature in each of the three battery compartments. Taking into account all these parameters gives a total of 24 different measurements that must be made from the batteries. Additionally the monitor must take account of the distance travelled by the vehicle in order to predict the remaining vehicle range available.

The monitor must have sufficient processing power to be able to process all of the data from the batteries and display this data to the user in a convenient form. Since time measurements are required in some of the monitor's calculations, the monitor must have an accurate onboard clock. It must also have some form of non-volatile data storage to store the various measurement parameters and data that must be retained when the monitor is powered down. In addition to these requirements the monitor must also be able to communicate with a host computer to allow downloading of logged data and updating of the monitor's measurement parameters.

\subsection{System Architecture}

Figure 5.1 shows a block diagram of the battery monitor hardware. Three Battery Measurement Modules (BMMs) make the various battery measurements. Each BMM is located in one of the three battery compartments which minimises the wiring connecting the batteries to the monitor. Eight measurement channels are contained on each BMM which are used to measure a combination of terminal voltage, temperature and battery current. The analogue to digital conversions are performed onboard each BMM and the measurements are sent back to the monitor system board as digital signals. This minimises the likelihood of noise and interference corrupting the measurements.

All data processing is performed on the monitor system board. This board as well as receiving data from the BMMs also receives pulses from the car's tachometer. The system board provides power supplies for the BMMs and displays and also provides 
interfacing between the monitor's microcontroller and a host computer. It also sends the processed information to the dashboard displays.

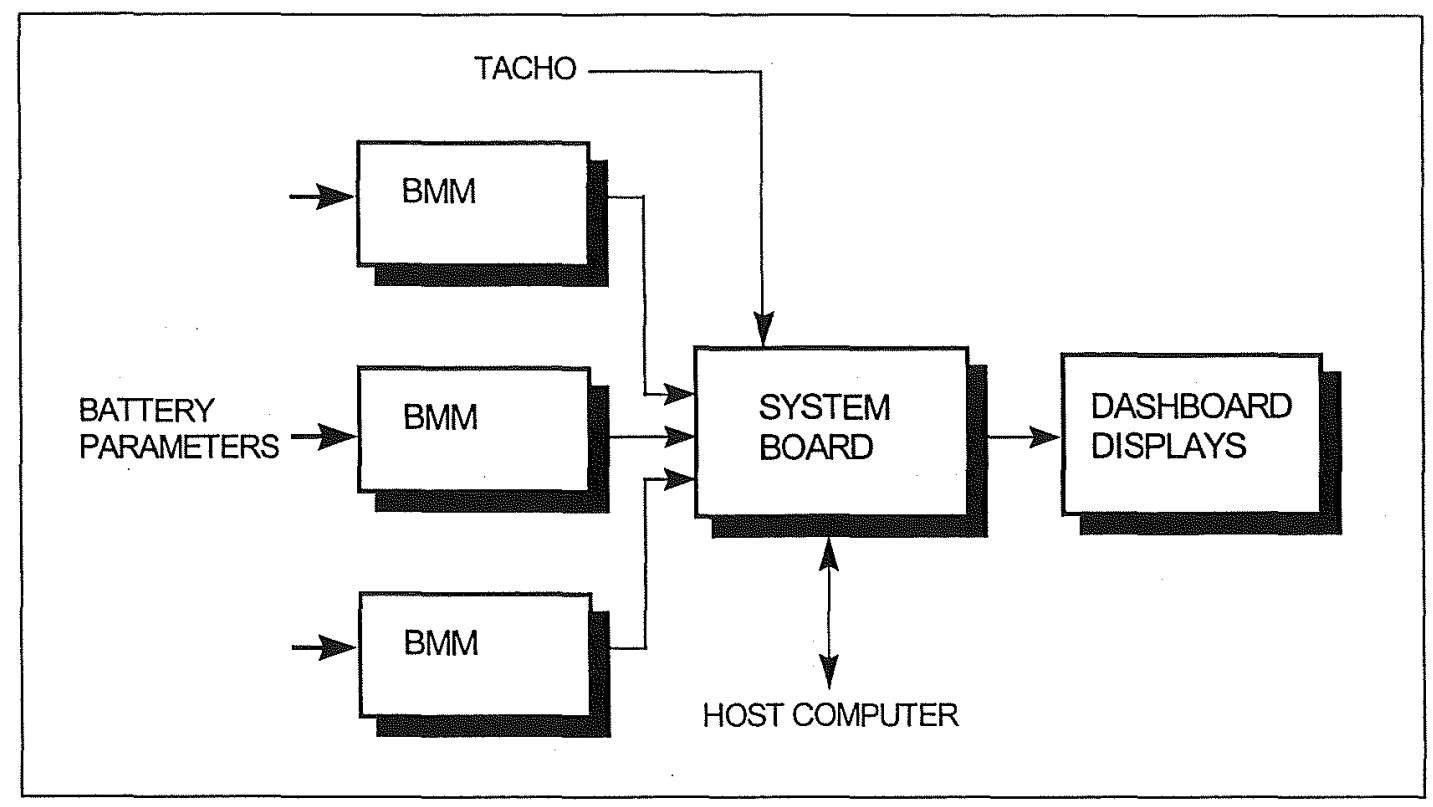

Figure 5.1: Block diagram of the battery monitor hardware.

\subsection{Battery Measurement Modules}

Each BMM has eight measurement channels and hence can measure eight parameters. The 24 different battery parameters are split between the BMMs depending on the location of each BMM. The first BMM is located in the battery compartment under the bonnet. Since this compartment contains eight batteries this BMM has been designed to measure just the eight terminal voltages present. The second BMM is located under the left seat. It measures the six battery terminal voltages, the temperature in that compartment and the battery current. The third BMM measures the remaining six battery terminal voltages and the temperature in the third battery compartment under the right seat. It also measures the temperature in the battery compartment under the bonnet.

Each BMM contains an analogue to digital converter and analogue signal processing circuitry that amplifies and isolates, where necessary, the incoming analogue signals from the batteries.

\subsubsection{Analogue to Digital Converters}

Predicting the state of charge of the batteries using open circuit voltage measurements requires that the voltage measurements are made with a resolution of $5 \mathrm{mV}$ or better in order to produce accurate results.

The maximum battery voltage that needs to be measured will occur when the charger is on and the batteries are at full charge. This will typically be around $14.4 \mathrm{~V}$ at $20^{\circ} \mathrm{C}$. If the 
voltage measurement channels input range was designed to be from $0 \mathrm{~V}$ to $15 \mathrm{~V}$, an analogue to digital converter with a resolution of 12 bits would be required. However, since the end of discharge point of the batteries occurs at around $7 \mathrm{~V}$ with a $100 \mathrm{~A}$ discharge current, it is only necessary to measure the battery voltage between around $6 \mathrm{~V}$ and $15 \mathrm{~V}$. By reducing the measured input range, lower resolution $\mathrm{A} / \mathrm{D}$ converters can be used with a resolution of only 10 bits. 12-bit Maxim MAX186 A/D converters were chosen however since several manufacturer's samples of this part were on hand. It is also desirable to use an $\mathrm{A} / \mathrm{D}$ converter with more bits than required as the least significant bits have a tendency to get lost in noise.

The MAX186 is designed for data acquisition systems and contains a 12-bit A/D converter with an eight channel multiplexer [13]. It has an accurate internal reference and communicates over a SPI (Serial Peripheral Interface) compatible serial interface which can run at speeds of up to $4 \mathrm{MHz}$. The MAX186 consumes very little power, around $1.5 \mathrm{~mA}$ when operating, and can be placed into a power-down mode which draws only $2 \mu \mathrm{A}$. It can sample at rates of up to $133 \mathrm{kHz}$. In this application however since the data only needs to be updated once or twice a second, it will not be used at anywhere near this speed. The MAX186 datasheet is included in appendix D2.

Figure 5.2 shows the circuitry associated with the MAX186 in the BMMs. Each of the MAX186 input channels are driven through a $4.7 \mathrm{k} \Omega$ resistor to match the impedance of the MAX186 input circuitry. This value of resistance ensures that the track and hold capacitors inside the MAX186 do not discharge significantly when an A/D conversion is taking place. The remaining circuitry allows trimming of the internal reference via trimpot VR1 and provides sufficient supply bypassing for the device.

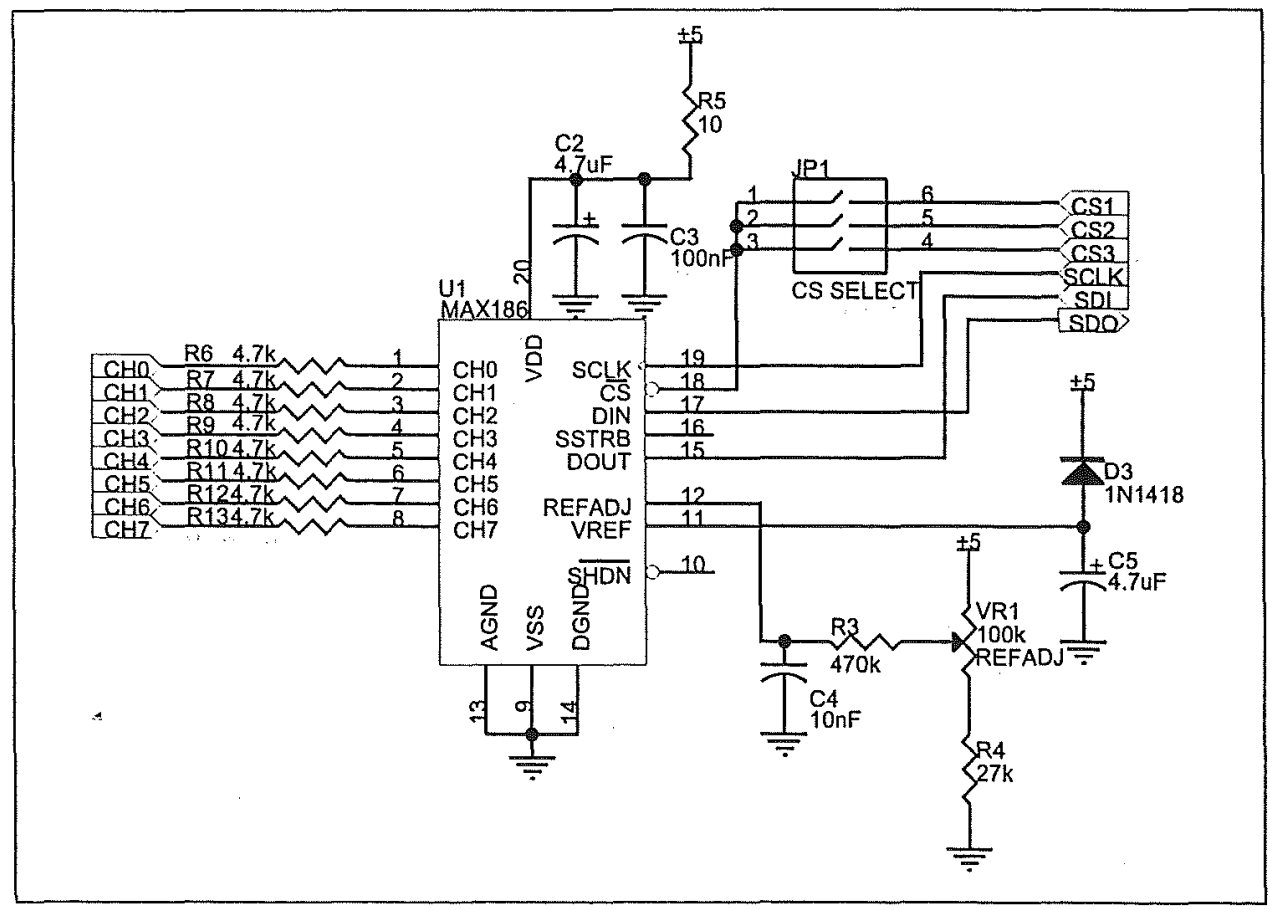

Figure 5.2: Circuitry associated with the MAX186 on the BMMs. 
The digital signals from the MAX186 are fed back to the main system board over an eight-wire cable. This cable provides power, serial clock, serial data in and out and three chip select lines for each of the three BMMs. The interface is daisy chained through the three BMMs. Jumpers are provided on each BMM to select which chip select line it is on.

To start a conversion an eight-bit command byte is sent from the main system board to the appropriate BMM. This command byte selects which channel the conversion is to take place on, defines the $A / D$ channel configuration and whether to power down the $A / D$ at the completion of the conversion. The A/D converter then responds by sending back two bytes of data that contain the 12-bit conversion result.

Power for the A/D converter and the other analogue circuitry on each BMM is supplied through a $5 \mathrm{~V}$ regulator on each board. The regulator helps stop any digital noise being induced in the BMM's power supply. This may occur since the power supply for the BMMs runs alongside the other digital lines in the same cable.

\subsubsection{Poll Indication}

The BMMs each have two LED indicators to show that they are operating correctly. The first is simply a power LED which is driven by the regulated 5V supply on each BMM board. The second LED indicates that the BMM is being polled by the microcontroller on the main system board. This LED is driven from the MAX186 chip select line on each board via a pulse stretching circuit.

The chip select line is pulled low by the microcontroller whenever it communicates with that particular device. The chip select line is typically only pulled low for around $40 \mu \mathrm{s}$ each time the BMM is polled. The pulse stretching circuit shown in figure 5.3 is used to extend the pulse duration to around $100 \mathrm{~ms}$, which is long enough to give a noticeable visual indication.

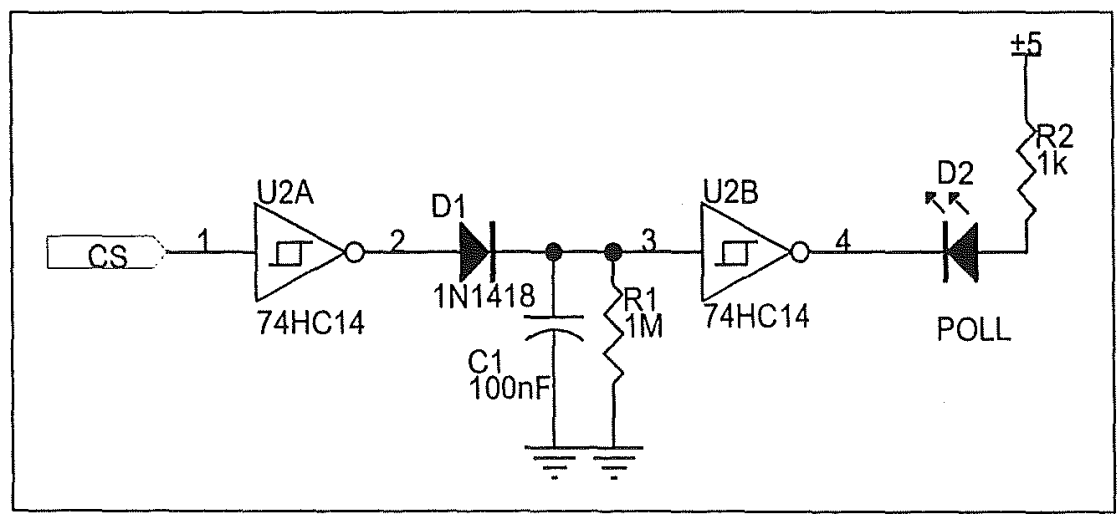

Figure 5.3: The pulse stretching circuit used to extend the chip select line pulse so that it can give a meaningful visual indication of the BMM being polled. 
The short duration pulse from the chip select line is inverted by the schmitt triggered inverter U2A. This allows the capacitor to charge through the diode. When the pulse is removed the capacitor discharges at a slower rate through the resistor in parallel with it. While the capacitor is charged it holds the input to the second inverter, U2B, high which results in a low at its output and hence lights the poll LED. When the capacitor discharges after around $100 \mathrm{~ms}$ the inverter output goes high and turns the LED off.

\subsubsection{Voltage Measurement Channels}

The car contains twenty $12 \mathrm{~V}$ batteries connected in series. Since there are high voltages of nominally $240 \mathrm{~V}$ involved in this configuration, it is difficult to directly measure the voltage across each battery. In order to make voltage measurements in a safe manner at these voltage potentials, some sort of isolation must be employed in the BMM input channels.

Isolation can either be provided by isolating the analogue signals before they are digitised or by isolating the digital signals after the digitising process. The latter of these methods is preferred as it is much easier to isolate digital signals without adding additional noise and distortion. However in this case each of the channels must be isolated from each other, so in order to use this isolation method would have required an individual A/D converter for each channel as well as three or more opto-couplers to isolate the digital signals coming from it. Providing isolation before the analogue to digital converter, although more difficult to do without distorting the signals, allows the use of a single, multi-channel analogue to digital converter and was therefore chosen for this application.

Figure 5.4 shows the block diagram for a single voltage measurement channel. The voltage across the battery is shifted to compress its range to between $6 \mathrm{~V}$ and $15 \mathrm{~V}$ and is passed into an isolation amplifier. The output of which is amplified so that it is a level suitable for the A/D converter's input. Power to the isolated section of the isolation amplifier is derived from the battery being measured.

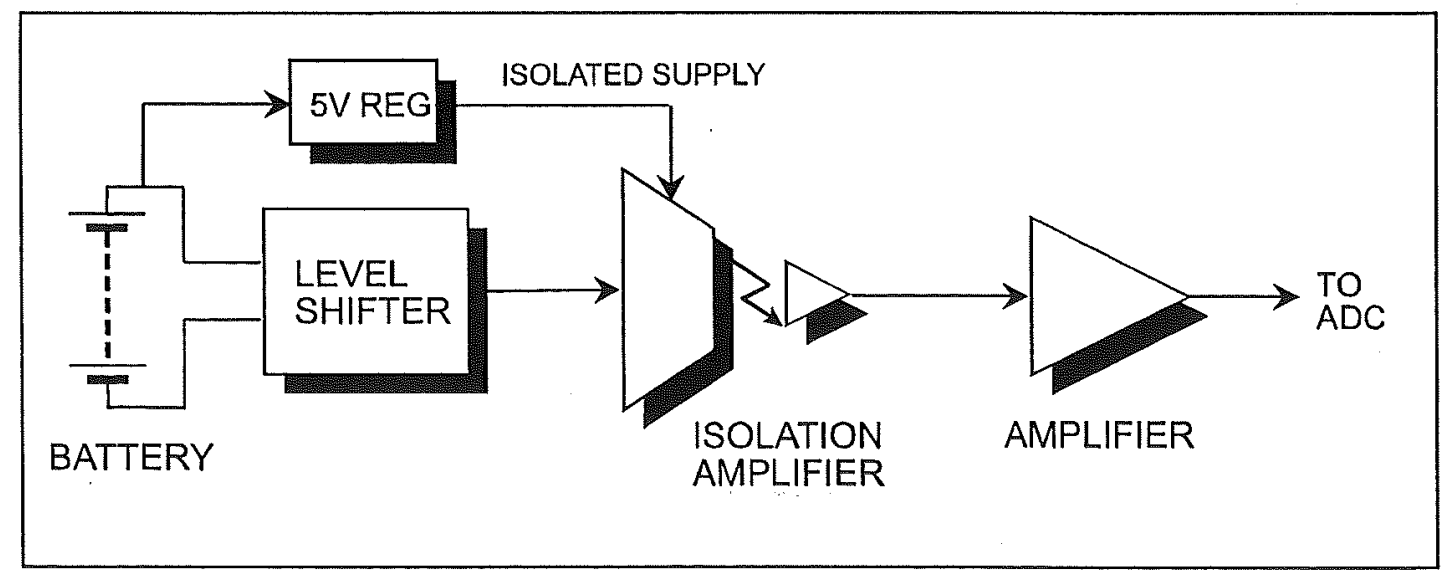

Figure 5.4: Block diagram showing the signal processing components of a voltage measurement channel. 
HCPL-7800 isolation amplifiers from Hewlett Packard were used instead of standard opto-couplers as standard opto-couplers tend to have poor linearity and significant drift with temperature. Figure 5.5 shows the internal block diagram of the HCPL-7800.

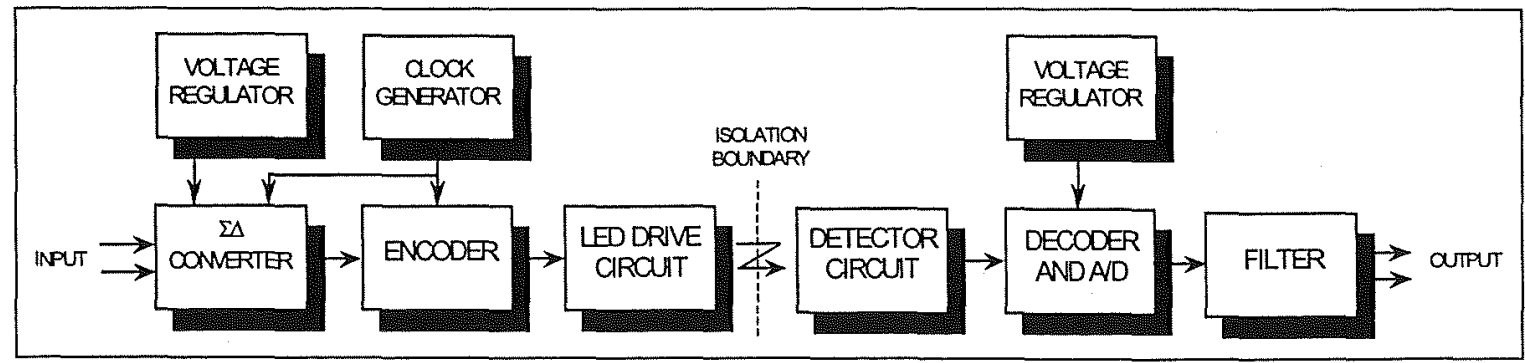

Figure 5.5: HCPL-7800 block diagram.

The HCPL-7800 does not work in the same way as a conventional opto-coupler. It utilises a sigma-delta analogue to digital converter to convert a differential analogue input signal to a digital one. This digital signal is then transmitted over an isolation boundary using a high speed LED and photo detector. The isolated digital signal is converted back to a differential analogue signal that appears at the device's output. Since the isolation is done digitally the device maintains very high linearity, has good common-mode rejection and a very low temperature drift. The datasheet for this device is included in appendix D3.

Figure 5.6 shows the circuitry used for one voltage channel. The isolation amplifier, U2, has a positive input range of between 0 and $200 \mathrm{mV}$. However for best linearity from the device this input range should be limited to $100 \mathrm{mV}$. The voltage across the battery being measured is therefore divided down by a factor of 90 before reaching the positive input of the isolation amplifier. The adjustable voltage divider made up from components, R1, VR1 and R2, perform this function and allow the input gain to be trimmed accurately.

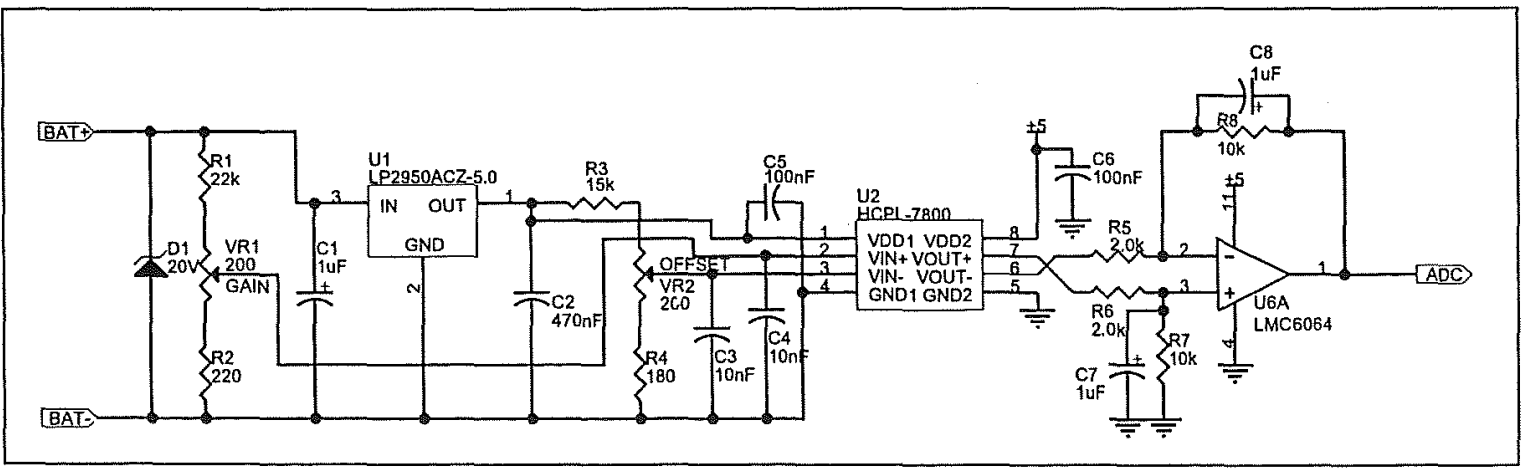

Figure 5.6: Circuit diagram of a single voltage measurement channel input and isolation circuitry.

The $5 \mathrm{~V}$ isolated supply to the isolation amplifier is derived from the battery being measured. This supply is regulated by U1, a low dropout precision voltage regulator, and is also divided down by a second adjustable voltage divider network consisting of 
components R3, VR2 and R4 to provide an offset voltage to the negative input of the isolation amplifier. This allows the offset to the amplifier to be adjusted so that the input range is effectively shifted so that it is between $6 \mathrm{~V}$ and $15 \mathrm{~V}$.

The isolation amplifier has a gain of around eight so the differential output from the isolation amplifier ranges from 0 to $800 \mathrm{mV}$. Opamp U3 is configured as a differential amplifier with a gain of 5.1 and is used to amplify the output of the isolation amplifier to give a resulting signal that ranges between $0 \mathrm{~V}$ and $4.096 \mathrm{~V}$. This is the input range of the analogue to digital converter. The differential amplifier has a low pass filter characteristic due to capacitors $\mathrm{C} 7$ and $\mathrm{C} 8$ connected across the feedback resistors which helps to provide filtering of unwanted noise that may be present on the battery voltages.

An LM6064 quad CMOS precision opamp was used for U3. The LM6064 is designed for signal rail operation and features a rail-to-rail output swing which allows it to be powered from the single 5V supply on the BMM board without the need for additional negative rail supplies

The $20 \mathrm{~V}$ zener diode connected across the input to the channel is used as a protective device. Under normal circumstances this diode will not conduct. However if the input voltage rises above the breakdown voltage of the diode or the battery is connected with an incorrect polarity it will conduct. This will cause a current to flow which will be large enough for the fuse connected in-line with the leads connecting to the BMM to blow and thereby protect the input circuitry against both excessive and reverse voltages.

\subsubsection{Current Measurement Channel}

The current drawn from the battery bus can range from -100A (when the car is braking) to $+100 \mathrm{~A}$ (when the car is accelerating). The current measurement channel must be able to measure currents over this range.

The car has an analogue current meter located on the dash, which is driven by a Hall effect based current sensor as shown in figure 5.7. These sensors are made by HEME and can measure bipolar currents between $-200 \mathrm{~A}$ to $+200 \mathrm{~A}$. They include onboard signal processing circuitry that produces an output voltage that is proportional to the current flowing through the sensor. This output signal ranges from $-10 \mathrm{~V}$ to $+10 \mathrm{~V}$. Since this sensor already exists in the car and is suitable for use with the battery monitor it is also used as the sensor for the current measurement channel. 


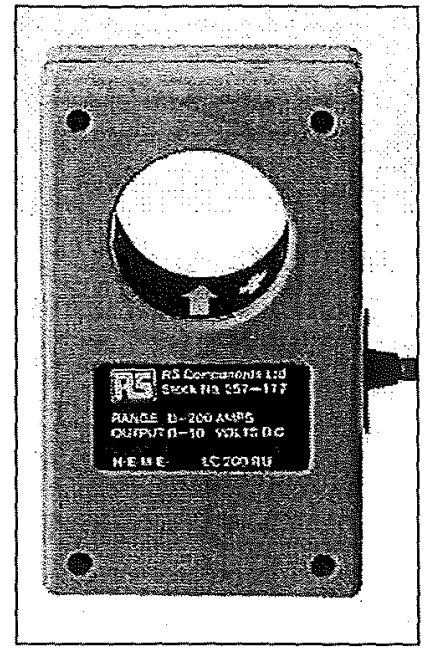

Figure 5.7: The Hall effect current transducer used for current measurement in the electric car.

Figure 5.8 shows the block diagram of the current sensor configuration and the signal processing of the current measurement channel. The sensor requires a $+/-15 \mathrm{~V}$ which is provided by an existing $1 \mathrm{~W} 5 \mathrm{~V}$ to $+/-15 \mathrm{~V}$ DC-DC converter located on the board on the rear of the analogue current meter. This supply is also used as a dual rail supply for the opamps in the current channel signal processing stage on the BMM board. The signal processing stage shifts the output of the current sensor so that it is all positive and reduces the amplitude of the signal so that it fits within the range of the analogue to digital converter.

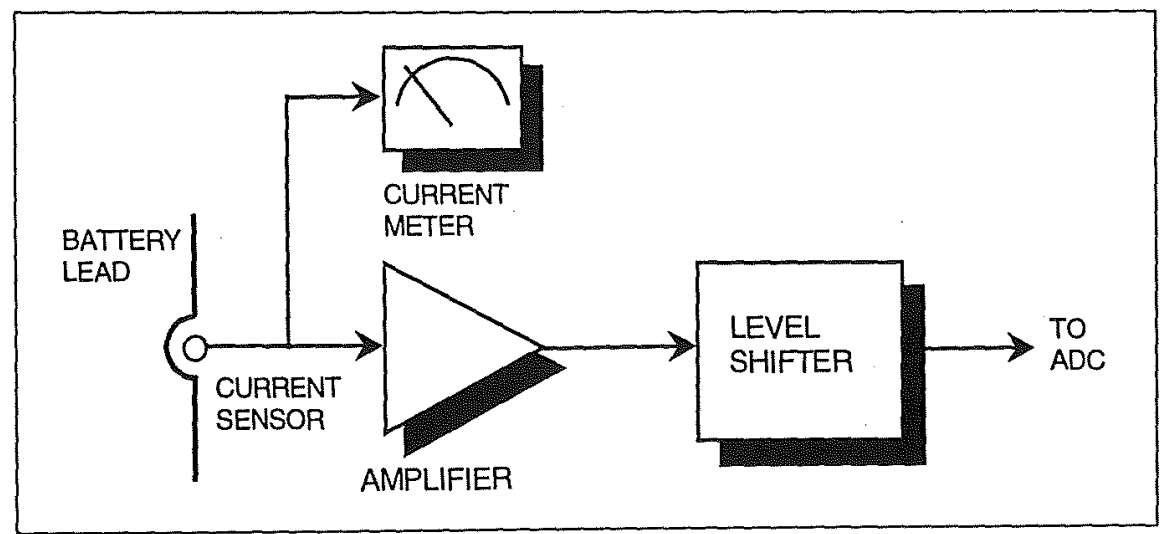

Figure 5.8: $\quad$ Block diagram of the current sensing and associated signal processing.

Figure 5.9 shows the gain and offset circuitry of the current input channel. The signal from the sensor first passes through U1a which is configured as an inverting amplifier with a gain of around 0.4 . The output of this stage gives a signal which ranges from $-2.048 \mathrm{~V}$ to $+2.048 \mathrm{~V}$ for an input signal ranging from $-5 \mathrm{~V}$ to $+5 \mathrm{~V}$. This corresponds to a measured current between $-100 \mathrm{~A}$ and $+100 \mathrm{~A}$.

The signal is shifted by a second inverting amplifier, based around U1b, with a gain set to one. The output shifting is achieved by offsetting the voltage on the non-inverting input 
of the amplifier. An adjustable reference formed from the voltage divider consisting of components R4, VR2 and R5 is used to supply this offset voltage. After passing through this stage the signal ranges from $0 \mathrm{~V}$ to $4.096 \mathrm{~V}$ with $2.048 \mathrm{~V}$ corresponding to 0A. This signal is fed directly to the A/D converter. Due to the fact that a 12-bit analogue to digital converter is used, the resulting digital measurements from the BMM have a minimum resolution of $50 \mathrm{~mA}$.

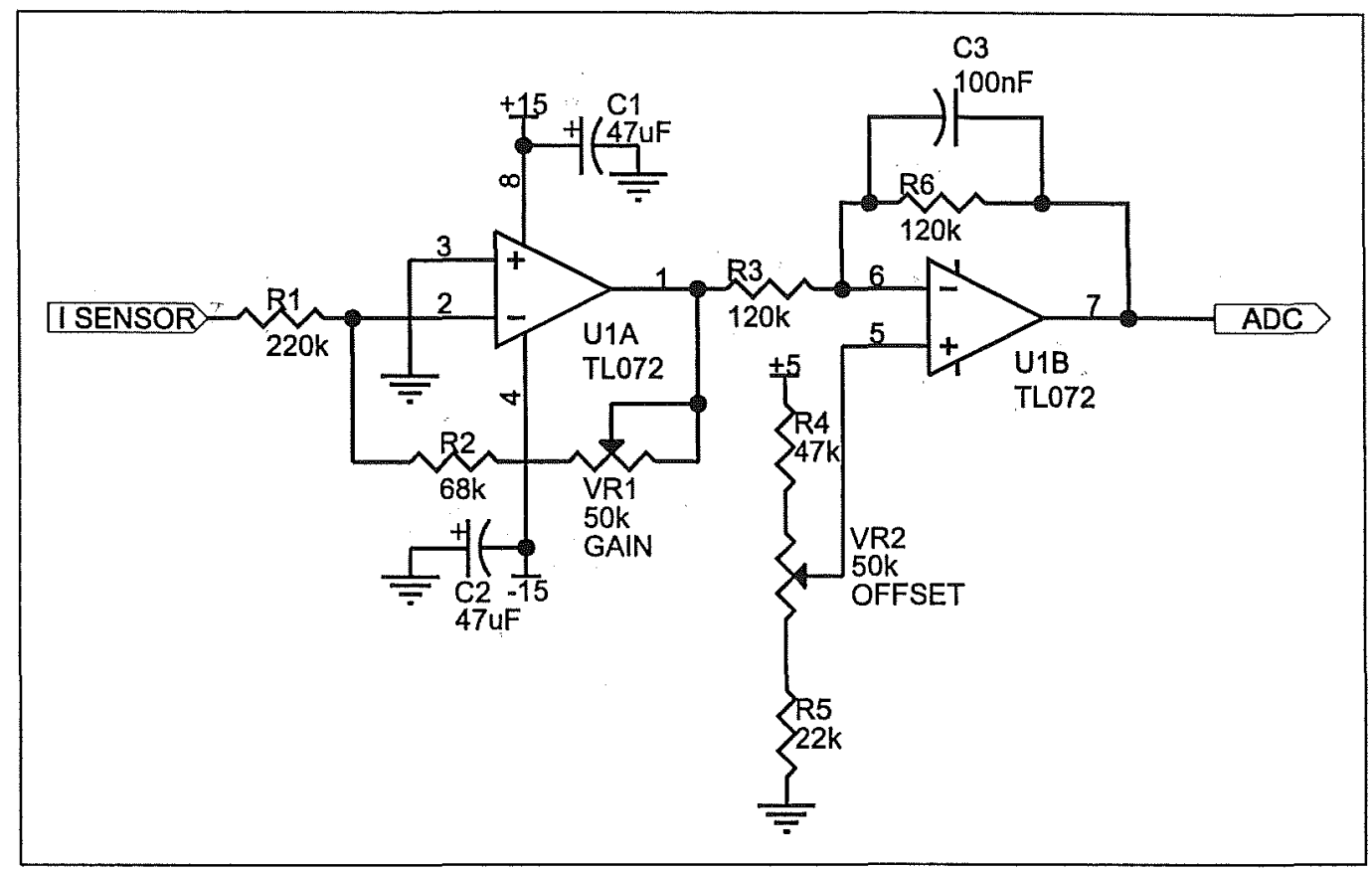

Figure 5.9: Current channel processing circuitry.

\subsubsection{Temperature Measurement Channels}

The temperature measurement channels are the simplest of the three types of channels on the BMMs. Figure 5.10 shows the block diagram of one temperature measurement channel.

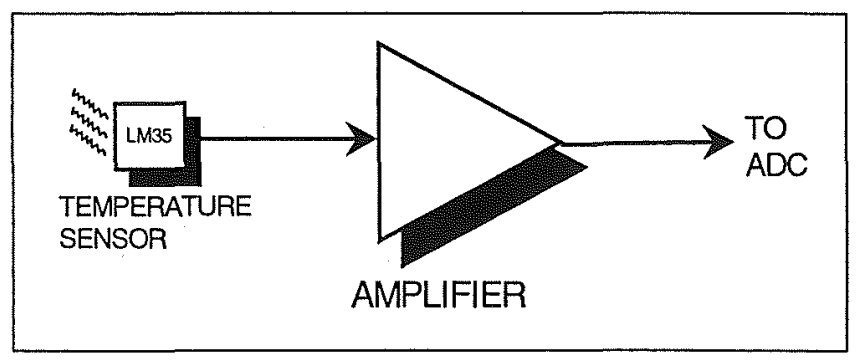

Figure 5.10: Block diagram of the BMM temperature measurement channel.

Temperature is sensed by an LM35 precision temperature sensor. This type of temperature sensor can measure temperatures over the temperature range from $-55^{\circ}$ to + $150^{\circ} \mathrm{C}$ and gives a voltage output that is directly proportional to the temperature. This 
signal must be amplified on the BMM boards to fit the full input range of the analogue to digital converter for best measurement accuracy.

Figure 5.11 shows the circuit diagram of a single temperature input stage. The input stage simply consists of a non-inverting amplifier based around a LM6061 opamp, U1. The gain of the amplifier is set to 4.096 so that the output from the temperature sensor, which ranges from $0 \mathrm{~V}$ to $1 \mathrm{~V}$, is amplified so that it fits the full input range of the analogue to digital converter.

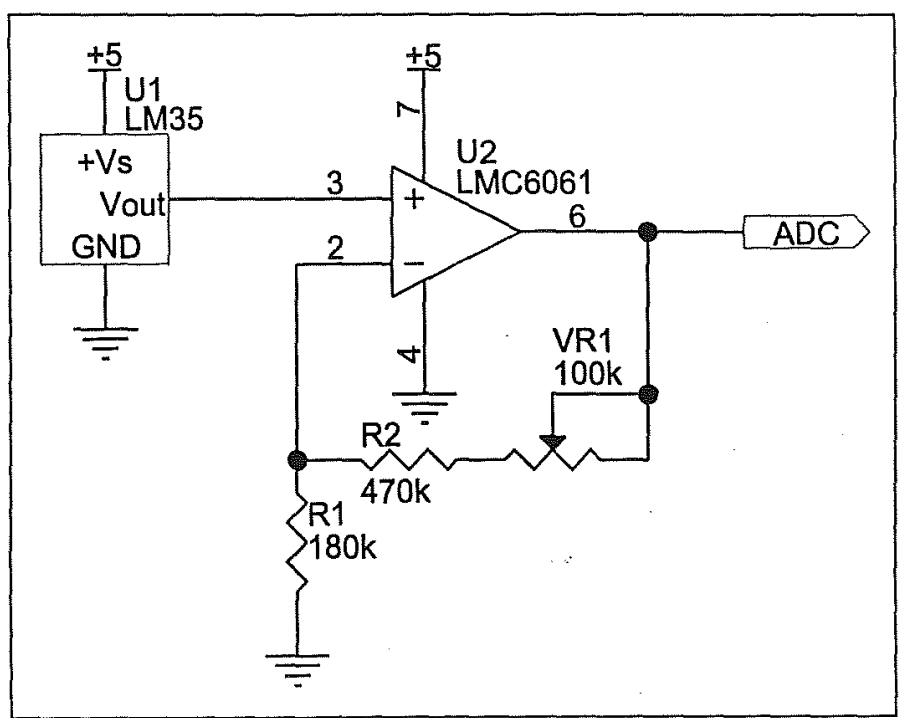

Figure 5.11: The temperature channel circuitry.

\subsubsection{Construction}

The full circuit diagrams for the three BMMs are included in appendices B1, B2 and B3. The three BMMs were constructed on three separate PCBs which are shown in appendices $\mathrm{C} 1, \mathrm{C} 2$ and $\mathrm{C} 3$.

The BMM PCBs contain both digital and analogue circuitry. This required careful layout of the boards to ensure that no digital noise was inadvertently coupled into the analogue circuitry. Separate ground planes were used for the digital and analogue sections on the board. Additional individual ground planes were used for each of the isolated voltage input stages since these had to be isolated from the main ground plane. Figure 5.12 shows one of the completed BMM boards. 


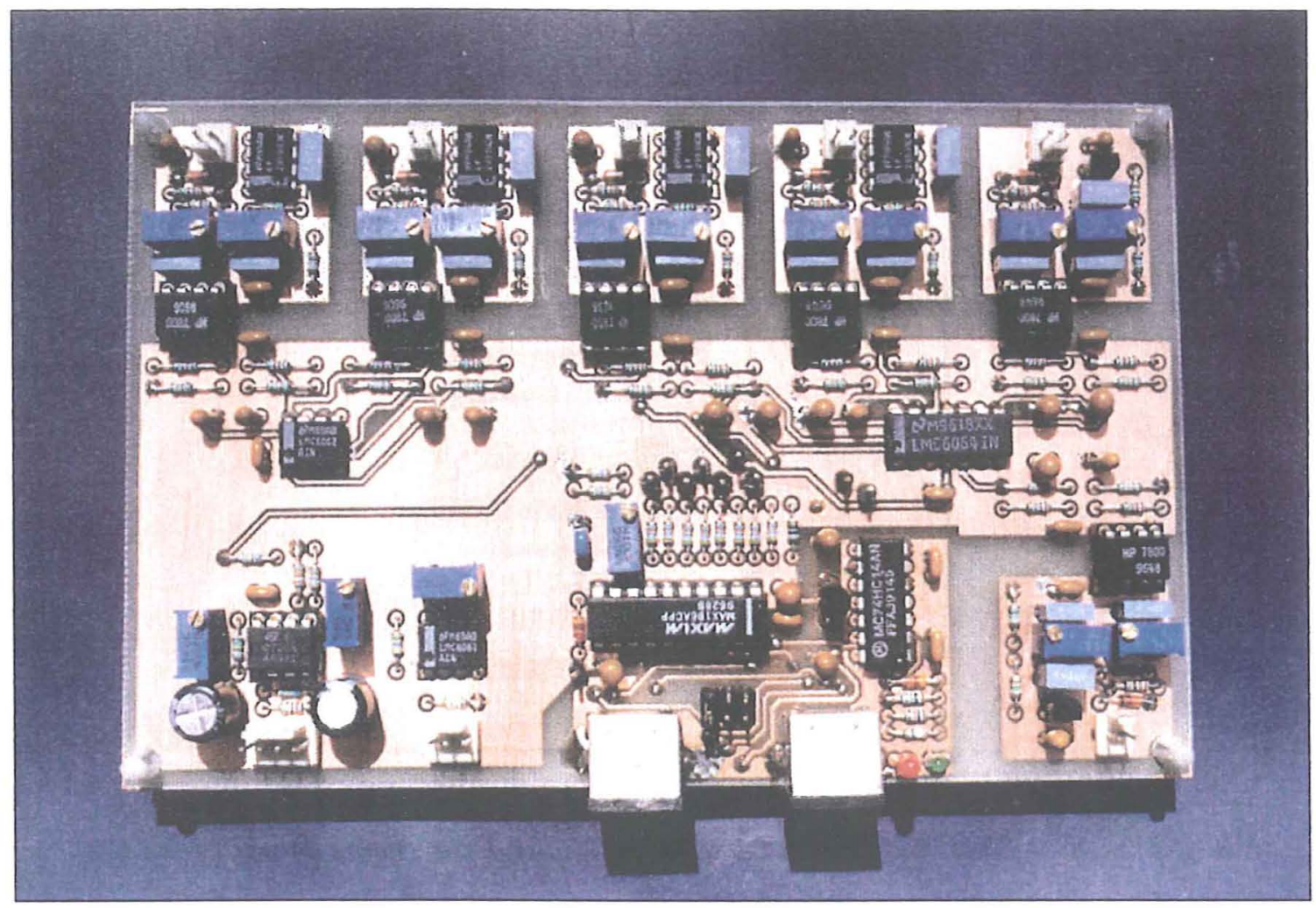

Figure 5.12: One of the completed BMM boards.

PCB mount 8-pin mini-DIN connectors were used for the serial interface bus connections. SIL headers were used for all the other connections to the board. These connect to multiway nylon connectors that connect to the battery leads as well as the current and temperature sensors.

\subsection{Battery Monitor System Board}

Figure 5.13 shows a block diagram of the battery monitor system board. The main component on this board is the microcontroller which performs all of the battery monitor's control and data processing functions. This board also contains a real-time clock, non-volatile data storage and an RS-232 interface. It also provides power supplies and interfacing to the other components of the battery monitor. 


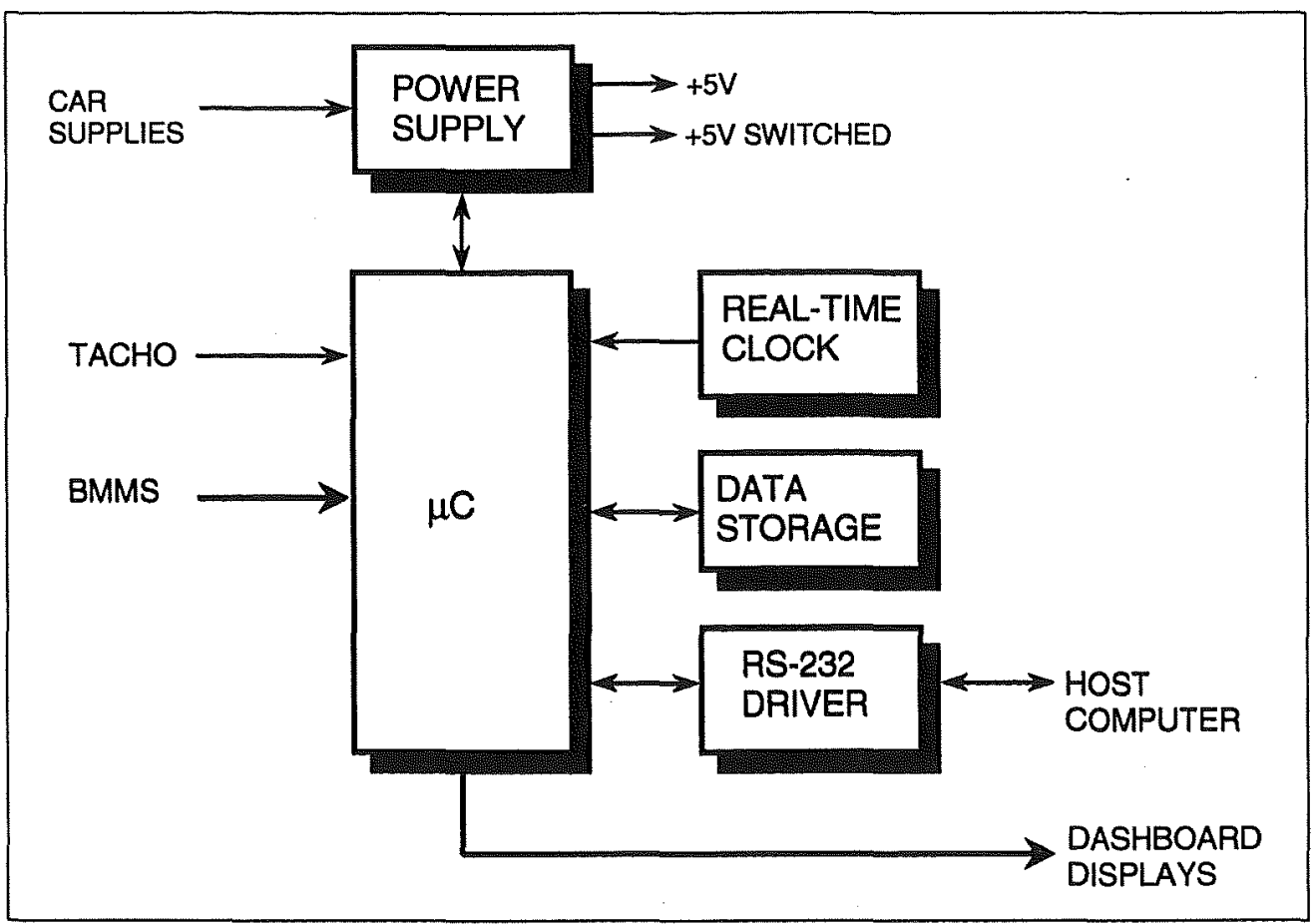

Figure 5.13: Block diagram of the battery monitor system board.

\subsubsection{Power Supplies}

The battery monitor has been designed so that it can be powered from several sources. While the car is running, power can be supplied from the base drive power supply for the car's inverter. When the car is being charged, since a $240 \mathrm{~V} \mathrm{AC}$ supply is available, power can come from this through a suitable step down transformer. The battery monitor also needs power for a short time after the car is switched off to gather open circuit voltage measurements and also requires a backup supply for the real-time clock. To allow for this, provision has been made for the battery monitor to be powered from a low power battery supply.

Figure 5.14 shows the circuitry associated with the battery monitor's power supply. $12 \mathrm{~V}$ supplies from both the car's base drive supply and the charger supply pass through an onboard fuse and diodes D1 and D2. These diodes, as well as providing reverse voltage protection, also automatically switch to the incoming supply with the highest voltage present. Supply filtering is provided by $\mathrm{C} 1$ and over-voltage protection is provided by $\mathrm{D} 4$, an $18 \mathrm{~V}$ zener diode. A battery supply of around $6 \mathrm{~V}$ or more can be connected to connector J2. Diode D5 provides switching for the battery supply. 


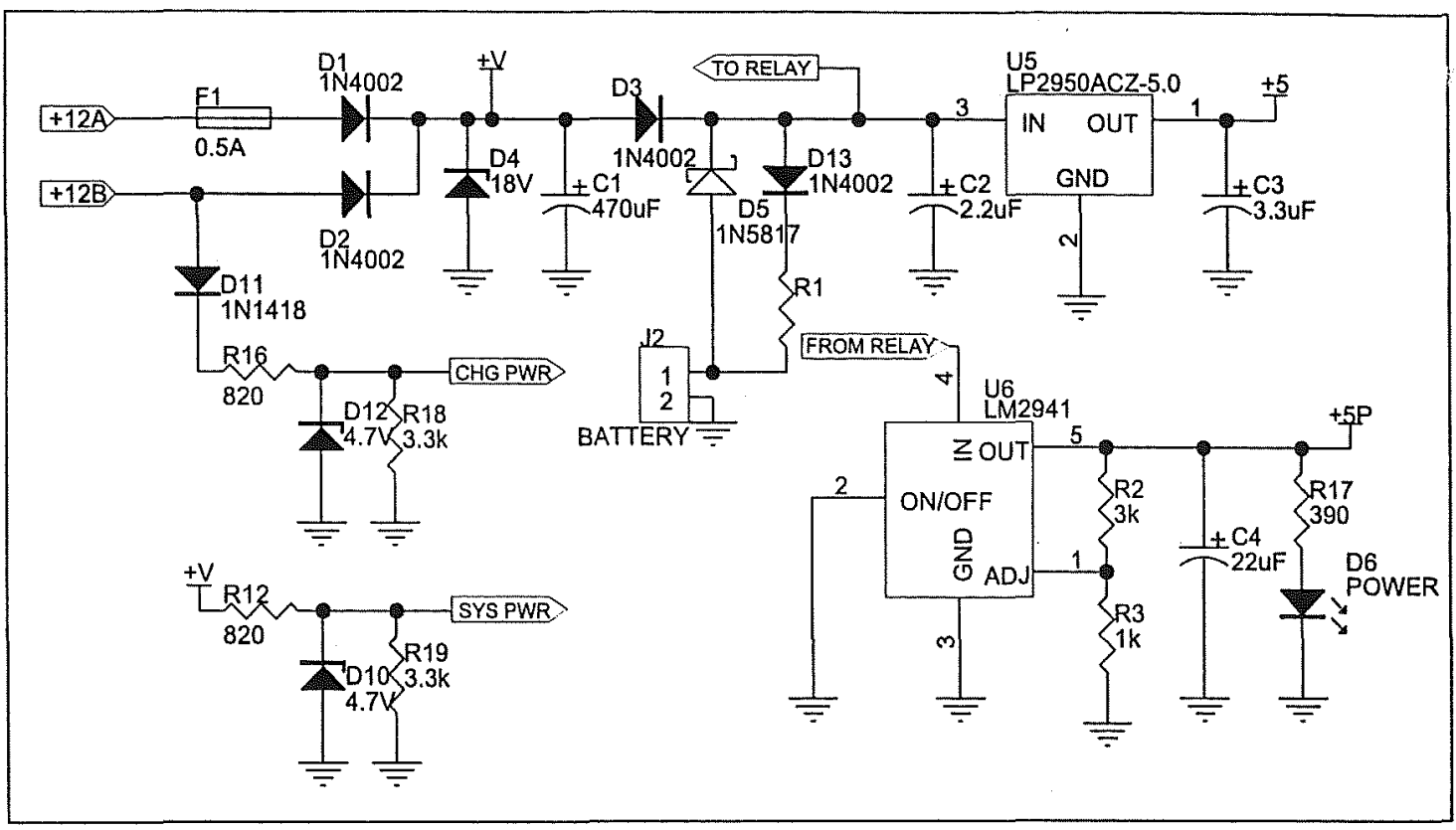

Figure 5.14: The battery monitor power supply circuitry.

Since many of the devices on the battery monitor system board have the facility to be placed into a low power shut down mode, two $5 \mathrm{~V}$ power supplies have been provided. $\mathrm{U} 5$ is a $100 \mathrm{~mA}$, low drop out regulator which provides an unswitched $5 \mathrm{~V}$ supply to power these devices.

The second supply is based around U6, a 1A, low drop out regulator which provides the switched supply for the BMMs and display boards. The regulator used for this has a logic level input which can be used to switch the regulator on and off. It was found however that the regulator consumed excessive current while it was shut down so the supply to the regulator is instead switched by a relay on the LCD display board. The switching of this supply is controlled by the microcontroller

The power supply also provides two logic signals which are used by the microcontroller to figure out which power supply is powering the monitor and hence know whether the batteries are being charged, discharged or in an idle state. One logic signal comes from the charger supply that tells the microcontroller that the charger is on. The second signal is derived from the unregulated supply into the monitor. This indicates that system power is available and hence the monitor is either running on the charger or base drive supplies. This signal also serves a second function. It is used by the microcontroller to generate an interrupt when power is restored to the monitor and hence wake the microcontroller up from sleep mode. The signals from the supplies are converted to logic levels through limiting resistors and $4.7 \mathrm{~V}$ zener diodes. Pulldown resistors are also necessary and are connected across the zener diodes. 


\subsubsection{Microcontroller}

Since the battery monitor interfaces to many different peripherals a microcontroller had to be selected that contained an onboard SPI compatible synchronous serial port, an RS-232 compatible serial port and sufficient $\mathrm{I} / \mathrm{O}$ lines to connect to the remaining monitor devices. In addition to these features it also needed to have sufficient program memory to contain all the required algorithms.

Three microcontrollers meeting these requirements were considered for use in the battery monitor. These were the Motorola 68HC11, the Siemens $80 \mathrm{C} 166$ and the PIC16C74 from Microchip. All of these microcontrollers would have been suitable for the task but since development tools and support were more readily available for the PIC16C74 this was chosen.

The PIC16C7X family of microcontroller are RISC based, low-cost 8-bit microcontrollers [14]. They are based on the Harvard architecture and use only 35 different 14-bit instructions. They contain a two stage instruction pipeline that allows most instructions to execute in a single clock cycle. The PIC16C7X series is designed to consume as little power as possible and can be put into a sleep mode which drops their supply current down to less than $1 \mu \mathrm{A}$.

The PIC16C74 contains 192 bytes of RAM, 4K of EPROM program memory and has 33 I/O lines. It also contains two serial ports, three timers and an onboard 8-bit A/D converter. The two serial ports include both a synchronous serial port which can be configured as either a SPI or $\mathrm{I}^{2} \mathrm{C}$ bus, and a Universal Synchronous Asynchronous Receiver Transmitter (USART) which can be used as an RS-232 interface.

The PIC16C74 can operate at clock speeds of up to $20 \mathrm{MHz}$. A clock speed of $8 \mathrm{MHz}$ was chosen for this application. This allowed the SPI interface, which has its clock derived from the main clock, to operate at $2 \mathrm{MHz}$.

Two of the three timers on the PIC16C74 can be configured as counters and clocked from an external source rather than the microcontroller's internal clock. One of these timers was used as a counter to count pulses from the car's tacho and is hence used to determine the distance the car has run.

One of the I/O ports on the PIC16C74 can be configured to generate an interrupt when a change in logic level is sensed on that line. One of these I/O lines is connected to the logic signal from the power supply that goes high when either the car or charger supplies is on. When power is restored to the battery monitor the change in logic level on this line will generate an interrupt which will wake the microcontroller up from sleep mode. 
Three more lines from this port are connected to the pushbuttons on the LCD display board so that an interrupt is generated when a button is pushed.

\subsubsection{Real Time Clock}

A 68HC68T1 CMOS Real-Time Clock (RTC) IC was used to perform the timing functions for the battery monitor. This real-time clock features an SPI serial interface, counts both time and date and can provide interrupts to the microcontroller either periodically or when a set time is reached.

The RTC uses a separate $32.768 \mathrm{kHz}$ crystal as an oscillator. Commands sent to the RTC over its SPI serial interface allow the date and time to be set and read, the RTC mode to be set and the chip to be placed into a shutdown mode. When it senses that system power has been regained the RTC automatically powers itself up again. Power to the RTC comes from the unswitched supply on the main board and it does not use a separate battery backup supply. The logic signal from the microcontroller used to switch the main power supply on and off is also applied to the system supply sense pin on the RTC to force it to power up when the microcontroller powers up the rest of the battery monitor.

\subsubsection{Data Storage}

The battery monitor requires non-volatile data storage to store the various measurement parameters, lookup tables used in the calculations and data that must be retained when the monitor is powered down. The most suitable type of memory to do this was found to be an $E^{2}$ PROM. An $8 \mathrm{k}$ X25642 $\mathrm{E}^{2} \mathrm{PROM}$ was chosen since it also uses a SPI interface to communicate with a microcontroller and its $8 \mathrm{k}$ of storage was considered to be more than adequate for the task.

This $E^{2} P R O M$ can be set into a low power standby mode so it is also powered from the unswitched monitor supply and is shutdown as part of the microcontroller's shutdown procedure.

\subsubsection{RS-232 Driver}

The USART on the PIC16C74 provides an interface that can be used to communicate with other RS-232 devices. The interface on the microcontroller however only provides transmit and receive lines that are compatible with TTL level signals rather than RS-232 levels. An RS-232 driver chip is therefore used to perform the level conversions.

The most popular chip used for RS-232 level conversion is the MAX232 from Maxim. In this case however a MAX233 chip was used since it does not require the four external capacitors that the MAX232 does and hence simplifies the circuitry. 


\subsubsection{Construction}

The circuit diagram of the system board is included in appendix B4. The PCB artwork for this board is shown in appendix C4. Figure 5.15 shows the completed board before installation into the car. Connection to BMMs and the RS-232 interface to the host computer are made through eight and four way mini DIN connectors located along the edge of the PCB. A header is provided for connection to a backup battery power supply. The remaining header connections on the board connect to the LCD display board.

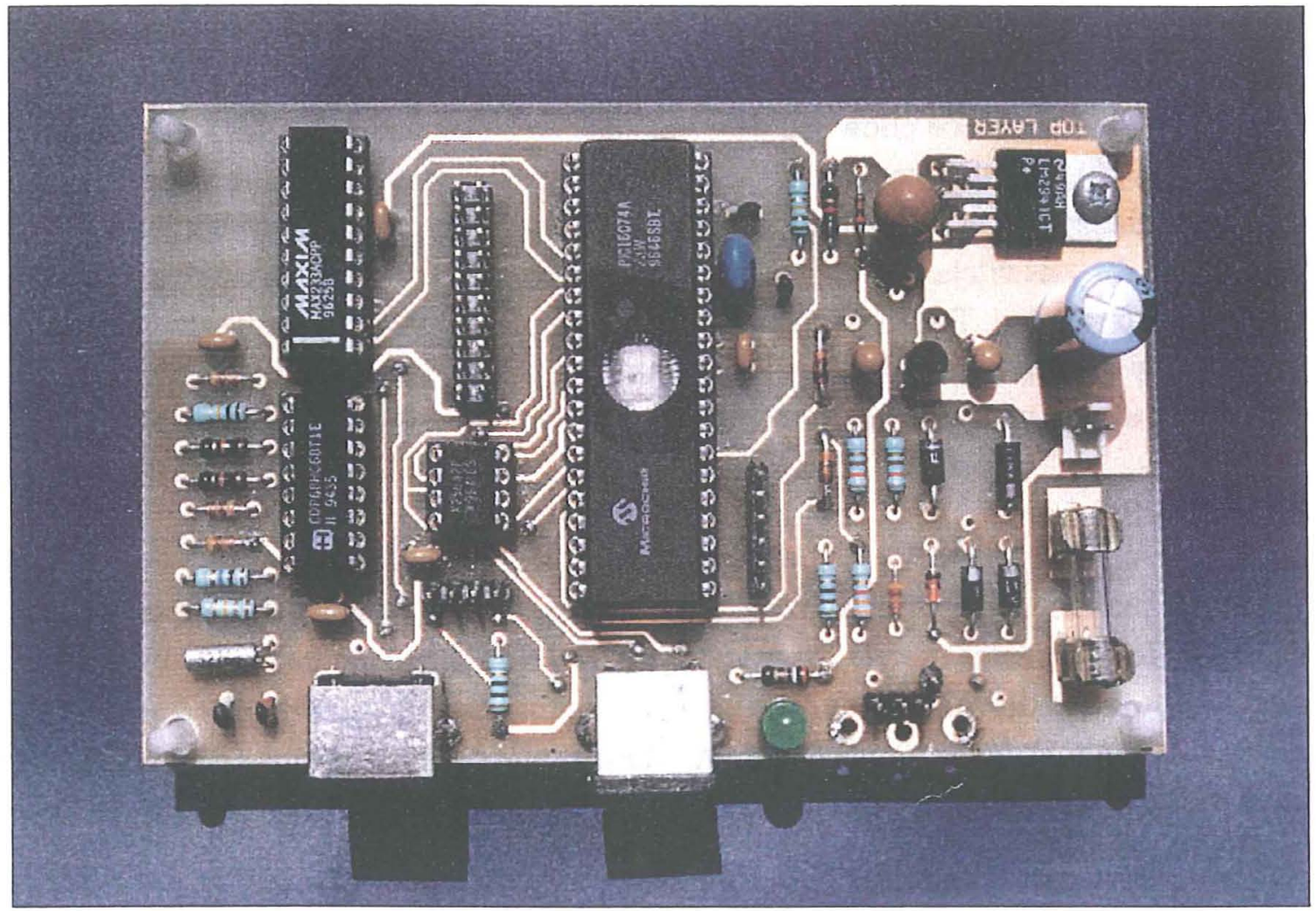

Figure 5.15: The completed battery monitor system board.

\subsection{Displays}

The battery monitor incorporates two in-vehicle displays. The first display is located with the other vehicle instrumentation on the dashboard and displays the state of charge of the batteries much like a conventional fuel gauge. The second display is based around an alphanumeric LCD panel and is used to display more detailed information about the state of the batteries and vehicle. As well as displaying the basic battery measurements, state of charge and state of health measurements, it also displays the distance that can be travelled on the remaining charge and also acts as a trip meter. 


\subsubsection{Information Display}

The information display is based around a twenty character by four line backlit LCD display. This type of display uses an industrial standard HD44780 chip set and contains a built in ASCII character set and display RAM. The LCD can be interfaced to a microcontroller over either a four or eight-wire data interface. An eight-wire interface was used in this case as spare $\mathrm{I} / \mathrm{O}$ on the microcontroller was available. Three additional signals are required for handshaking with the microcontroller. The LCD panel also accepts a variable DC input voltage to adjust the display contrast. A trimpot is used to allow this level and hence the contrast to be adjusted.

The LCD panel uses LED backlighting which draws around $800 \mathrm{~mA}$ at $5 \mathrm{~V}$. A separate power supply on the information display board provides power to the backlighting. This power supply is based around a LM317 adjustable regulator. A second trimpot is used to adjust the output voltage of the regulator and hence the display brightness.

Three pushbuttons are contained on the information display. These allow the user to scroll up and down through the various display screens as well as reset the trip meter. The three pushbuttons are debounced on the display board using a debounce circuit based around a schmitt triggered inverter, two resistors and a capacitor (figure 5.16).

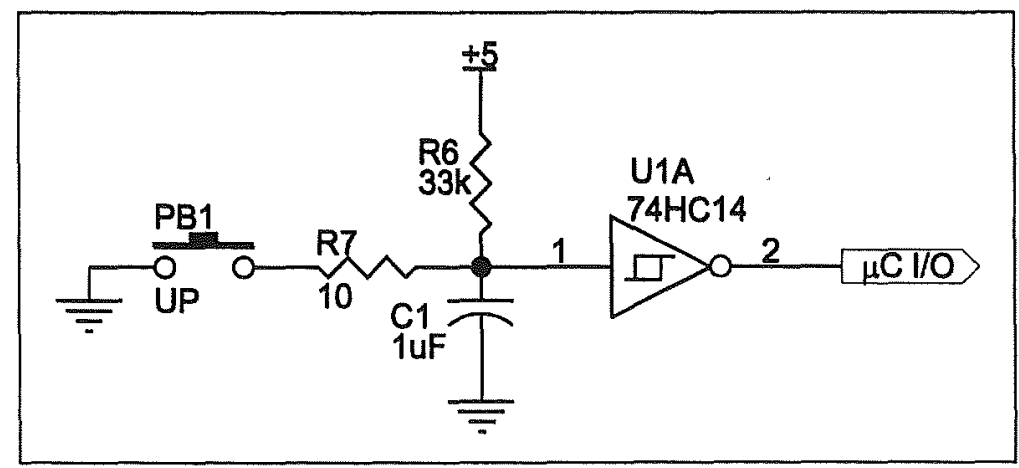

Figure 5.16: Pushbutton debounce circuit.

The debounce circuit works as follows. Before a button is pressed the capacitor is allowed to charge through R1 which pulls the input to the inverter high and hence the output low. When a button is pressed it discharges the capacitor through R2 pulling the input to the inverter low and hence the output high. The output of the inverter falls low again once the button is released and the capacitor charges up again. This prevents any switch chatter getting to the microcontroller.

The three signals from the debounce circuits are applied to $\mathrm{I} / \mathrm{O}$ lines on port $\mathrm{B}$ of the microcontroller. A change of logic level on these lines due to a button being pushed will generate an interrupt inside the microcontroller. 
The remaining circuitry on the information display board provides interfacing to the dashboard state of charge display, the vehicle's tacho and to the car power supplies. A signal from the car's headlights is also processed on this board. This signal is used by the microcontroller to sense night time use of the vehicle and hence allows the dashboard display to be dimmed. This signal has to be optically isolated from the battery monitor since the headlight supply uses a different ground to the supply used to power the battery monitor. The power switch relay is also contained on this board as mentioned in section 5.4.1. This board connects to the battery monitor system board via a 34 way ribbon cable.

The circuit diagram and PCB for the information display board is shown in appendices B5 and $\mathrm{C} 5$ respectively. Figures 5.17 and 5.18 show views of the completed display board.

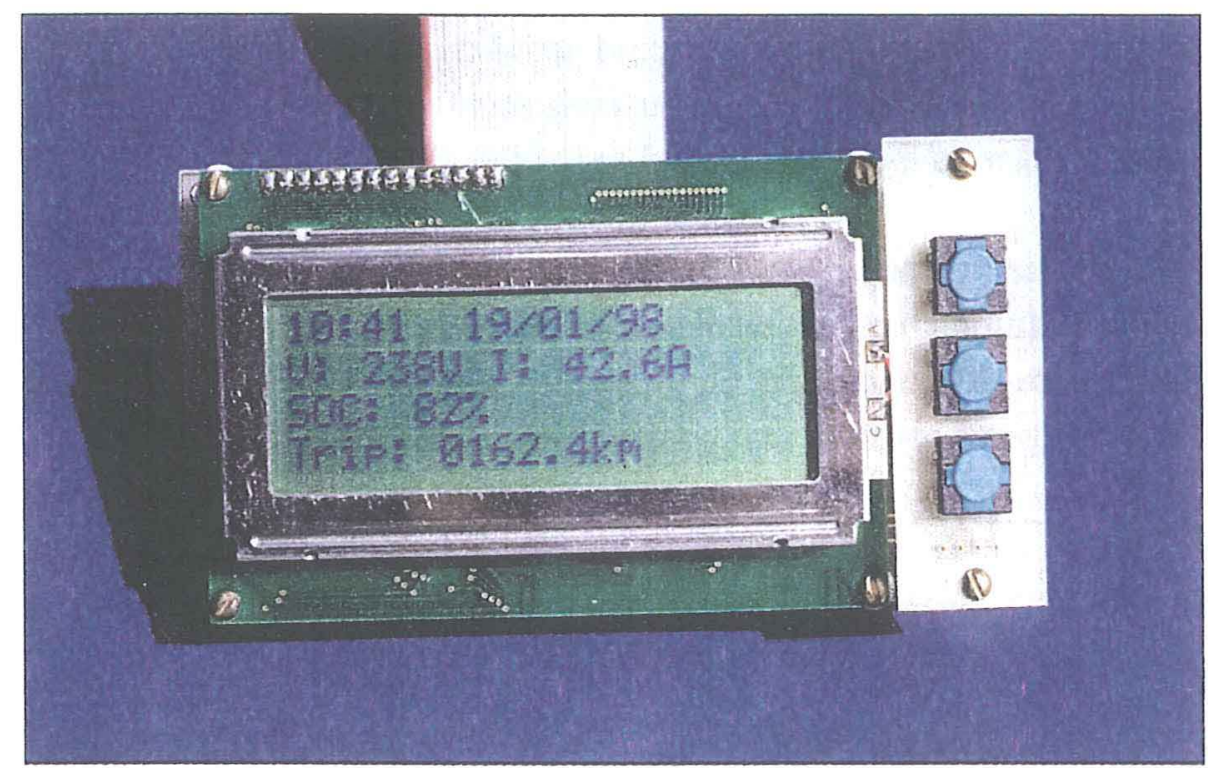

Figure 5.17: The front LCD display board with the LCD panel in place.

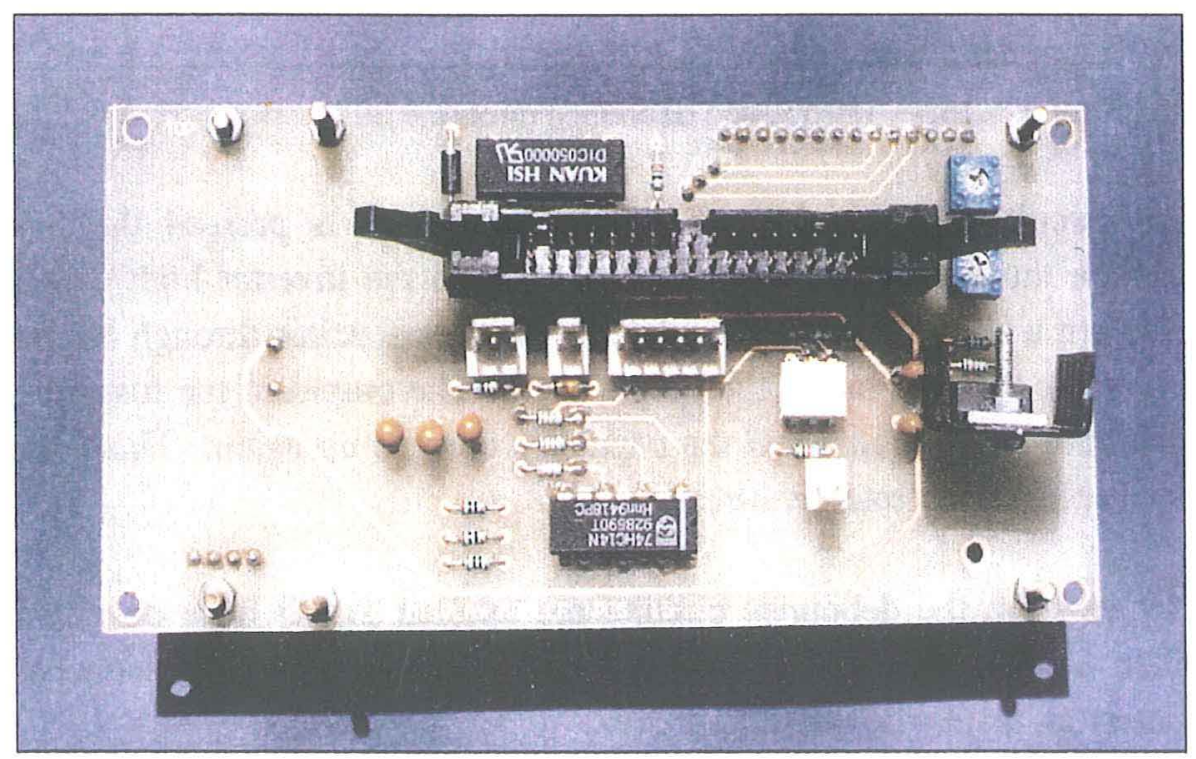

Figure 5.18: The rear of LCD display board where most of the circuitry is located. 


\subsubsection{State of Charge Display}

The state of charge display consists of a ten LED bargraph display designed to replicate the fuel gauge in a conventional vehicle. This display shows the state of charge of the batteries in $10 \%$ steps and also serves to provide a visual warning when the state of charge falls below $40 \%$. This is achieved by using bicolour LEDs for the 10, 20, 30 and $40 \%$ bargraph segments. Single colour green LEDs are used for the remaining segments. When the state of charge is above $40 \%$ the bicolour LEDs shine green to match the other LEDs. When the state of charge falls to $40 \%$ or below the bicolour LEDs are made to shine red to warn the driver of the limited remaining battery capacity.

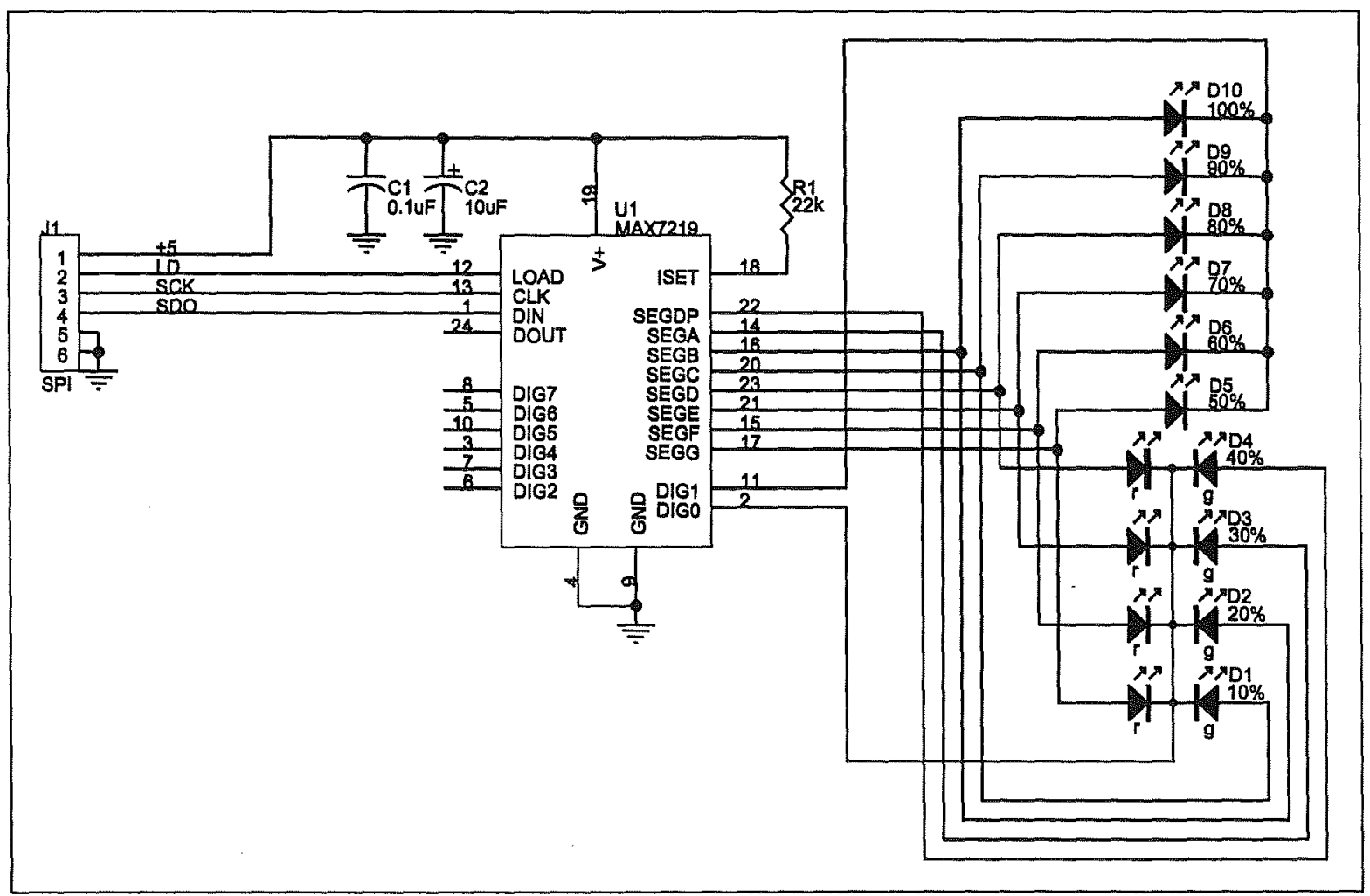

Figure 5.19: Circuit diagram of the state of charge display board.

Figure 5.19 shows the circuit diagram of the stage of charge display board. The ten LEDs are driven directly by a MAX7219 display driver chip from Maxim. This chip, although designed for driving up to eight 7-segment displays, also incorporates a 'no-decode' mode that allows it to address individual LEDs. The MAX7219 datasheet is included in appendix D4.

The display driver chip accepts commands over a three-wire serial interface that is compatible with the SPI used through out the rest of the battery monitor. Commands sent to the driver chip as well as telling it which LEDs to turn on also allow various operational modes and parameters to be set. Commands are available which allow the display to be put into a low power shutdown mode, set the intensity of the display and 
also test the display. Power for the display is supplied by the main regulator on the system board.

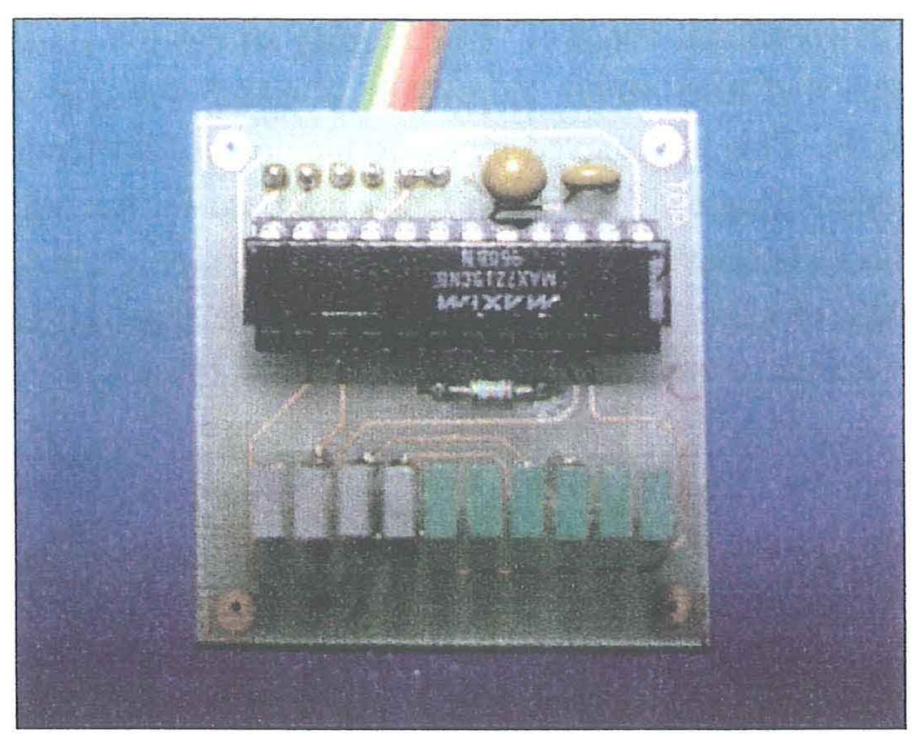

Figure 5.20: The state of charge display board.

Figure 5.20 shows the completed display board. The artwork for this board is also included in appendix C5. The board was designed to mount inside the existing instrument panel. To facilitate this the instrument panel was updated to replace the existing $12 \mathrm{~V}$ lamp based indicators with LED based ones. Also the trip meter was removed and replaced by a trip meter function added to the battery monitor to make additional room for the state of charge display. The circuit diagram and the PCB layout for the new indicators are shown in appendices B7 and C7. 


\section{System Software}

\subsection{Requirements}

The battery monitor software is responsible for the data processing and control functions of the battery monitor. These functions include taking measurements of the battery parameters and the tachometer at regular intervals, performing calculations on the measured data, and formatting the results in such a way that can be displayed on the car's state of charge meter and LCD panel (section 5.5). In addition to these functions, the software must also acknowledge when the push buttons on the front panel of the monitor are pressed, control the battery monitor power supplies, and communicate with a host computer over an RS-232 interface.

The battery monitor was designed so that all the data processing could be done by the microcontroller on the system board. This however required that all the battery monitoring and control algorithms were implemented in PIC assembly language which, considering the number of mathematical functions used in the monitoring algorithms, would be a time consuming task. It was therefore decided for development purposes to implement the monitoring algorithms in Visual Basic on a host computer and only provide a basic set of routines in PIC assembly code to drive the various hardware devices within the battery monitor and provide the interface back to the host computer. This approach allowed the battery monitor algorithms to be easily tested and modified without having to edit and debug assembly language code and hence helped simplify the software development process. Once the software is fully fine tuned and tested the algorithms can be transferred to an assembly language version.

\subsection{Microcontroller Code}

The PIC16C74 microcontroller described in section 5.4.2 is relatively easy to program as its instruction set contains only 35 instructions. These instructions provide most of the basic math and logical functions normally found in an eight bit microcontroller instruction set. Each instruction is 14-bit wide and contains the instruction and one or more operands.

In addition to the $4 \mathrm{k}$ of program (EPROM) memory, the PIC16C74 contains 192 bytes of data memory. Also mapped into the data memory area are 53 special function registers. These registers are used by the CPU and peripheral devices on the microcontroller. They allow the peripheral devices to be controlled as well as providing a path for data to be written and read from the peripheral devices. The PIC16C74 also contains an eight level 
stack that is used during subroutine and interrupt calls. There are no instructions provided to use the stack for other purposes.

Interrupts can be generated from 12 different sources which include the serial ports, the three timers, the onboard A/D converter, as well as from external sources. Each interrupt can be enabled individually. When an interrupt occurs, the interrupt service routine must check the interrupt flags to determine which interrupt has occurred. The microcontroller does not save the working or status registers when an interrupt occurs so this must be done in the program code.

Figure 6.1 shows the basic program flow of the microcontroller code. Full listings of the code are included in Appendix $\mathrm{E}$. The code occupies just under $1 \mathrm{k}$ of program memory.

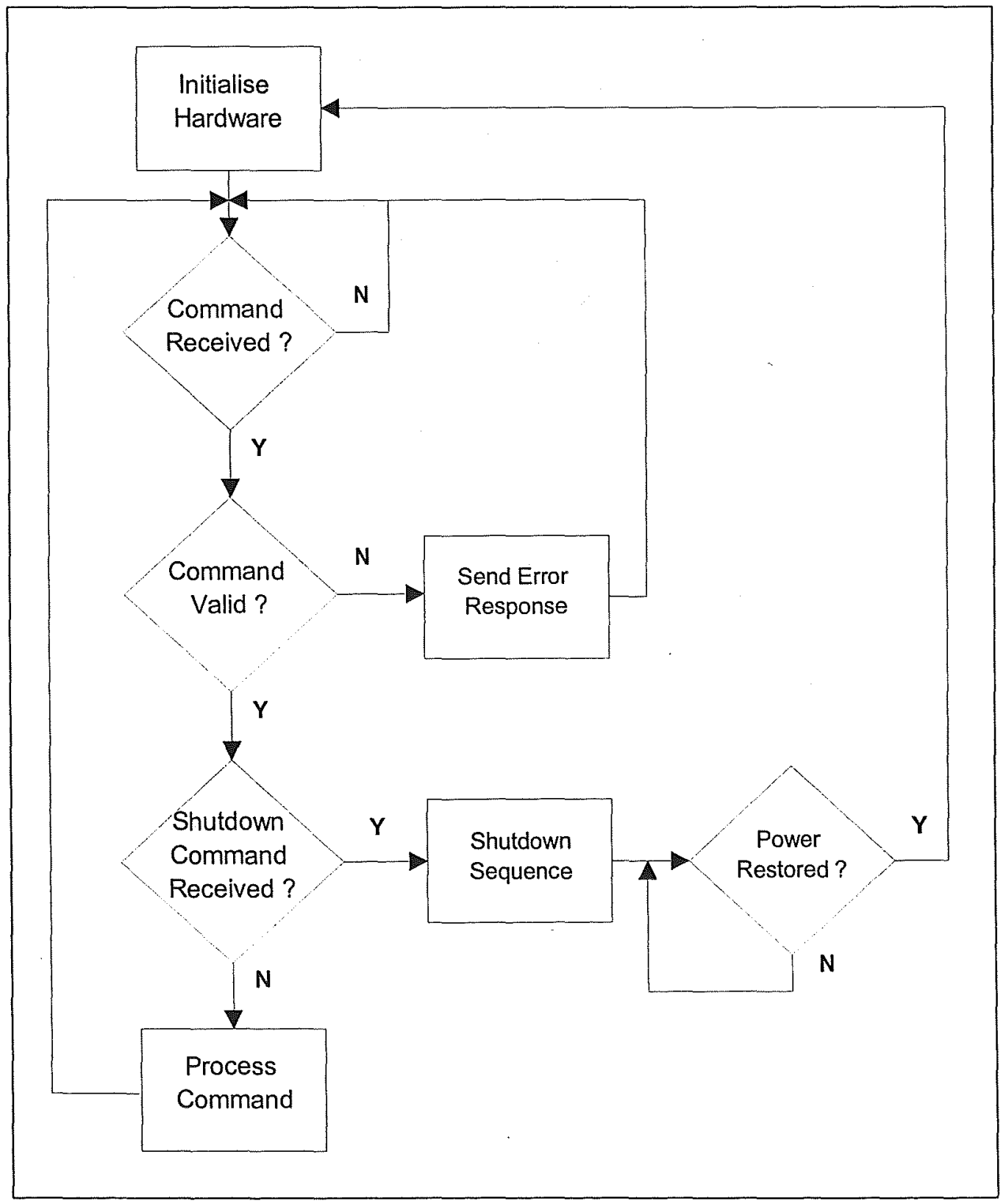

Figure 6.1: The basic program flow of the microcontroller code. 
On powerup or wakeup from sleep the registers within the microcontroller and the various monitor peripherals are initialised. The first part of this process involves setting up all the $\mathrm{I} / \mathrm{O}$ pins on the microcontroller as either inputs or outputs depending on their function, setting up the SCI port as an RS-232 compatible port running at 9600 bits per second and configuring the serial port as a SPI port running at $2 \mathrm{MHz}$. This is achieved by writing the appropriate information to the registers associated with each port. The port B change interrupt and the Timer0 overflow interrupt are also enabled at the point.

A counter, used to count the pulses from the tachometer, is also reset in the initialisation sequence and the various internal microcontroller data registers are initialised. The external real time clock (RTC) is checked to see if this is the first time it has been powered up and if it is the RTC control and interrupt registers are initialised to set the type of timing crystal used by the RTC and to set the RTC interrupt interval. The interrupts generated from the RTC are not used in this implementation but would be used to provide software timing in the final version of the software implemented entirely inside the microcontroller.

The LCD on the information display board is configured for an 8-bit interface and the display is cleared. Also the LED display driver for the state of charge meter on the dashboard is initialised to configure it as a bargraph driver. At the end of the initialisation sequence the software version is displayed on the LCD as well as a 'Waiting for host' message. The text strings for both messages are stored in the $E^{2} \mathrm{PROM}$ and are read out and sent to the LCD one character at a time.

After the initialisation process is complete, the microcontroller waits for a command to be received from the host over the RS-232 interface. When a byte is received it is checked to see if it is valid and if it is the command is processed by calling the appropriate subroutine. At the completion of servicing the command, with the exception of the shutdown command, the microcontroller returns to wait for the next command from the host. If the command is not valid, an '?' character is sent back to the host to signify that an error has occurred.

The battery monitor will respond to 16 different commands. Most of these commands can be divided into measurement, data transfer, display and control commands.

\subsubsection{Measurement Commands}

Two measurement commands have been provided which are summarised in table 6.1. The first is the BMM measure command which is used to initiate a signal measurement from the BMMs. This is achieved by sending the ASCII character ' $m$ ' to the battery monitor followed by a byte containing the channel number that the measurement is to be made from. 
As there are a total of three BMMs with eight channels each, there are 24 available channels numbered from 0 to 23 . On receiving the channel number the microcontroller calculates which of the three BMMs the channel is to be read from and also calculates the actual $\mathrm{ADC}$ channel on that BMM to get the measurement from. A lookup table is used to find the corresponding 8 bit instruction byte to send to the $\mathrm{ADC}$ on the BMM to start the conversion on that channel. Once the command has been sent, two bytes are received back from the ADC containing the conversion result. As the conversion result is only 12bits, four trailing zeros are added to the conversion result by the ADC. These are removed by shifting the conversion result four places to the right so that the conversion result has four leading zeros. The resulting 16 -bit word is sent to the host as two 8-bit bytes.

\begin{tabular}{|l|l|l|l|l|}
\hline Command & Character & Parameters & Returns & Description \\
\hline $\begin{array}{l}\text { BMM } \\
\text { Measure }\end{array}$ & $\mathrm{m}$ & $\begin{array}{l}<\text { channel \#> } \\
(0.23)\end{array}$ & $\begin{array}{l}\text { ADC data (16 } \\
\text { bits) }\end{array}$ & $\begin{array}{l}\text { Reads channel from ADCs specified in the <channel } \\
\text { \#> parameter. }\end{array}$ \\
\hline $\begin{array}{l}\text { Read } \\
\text { Tacho }\end{array}$ & $\mathrm{k}$ & - & $\begin{array}{l}\text { tacho count (24 } \\
\text { bits) }\end{array}$ & Read tacho counter and sends data back to host. \\
\hline
\end{tabular}

Table 6.1: Summary of the battery monitor measurement commands.

The second measurement command is used to read the tacho counter. The tacho counter is implemented using the Timer 0 module on the microcontroller configured as a counter. The counter receives a divided down pulse from the tachometer mounted on one of the motors. These pulses correspond to one pulse per every $10 \mathrm{~m}$ of travel by the car. As Timer0 is only 8 bits wide it can only count up to $2.56 \mathrm{~km}$ before it overflows. To overcome this a second register is incremented every time the Timer0 register overflows. These two registers makes the tacho counter effectively 16 bits wide so it allows a tacho count of up to $655.36 \mathrm{~km}$. The tacho count is however used to derive the car's trip meter which needs to be able to count up to $9999.9 \mathrm{~km}$. It was therefore necessary to use a third register that is incremented each time the second register overflows, making the tacho counter effectively 24 bits wide.

To detect each time Timer0 overflows the Timer0 overflow interrupt is used. When Timer0 overflows an interrupt is generated which causes the program to branch to the interrupt service routine. The interrupt service routine, after saving the current status of the main registers, checks to see if a Timer0 overflow has occurred. If it has the interrupt flags are cleared and the second tacho register is incremented by one. This register is checked to see if it has overflowed and if it has the third tacho register is incremented. The interrupt service routine also checks to see if any of the front panel buttons have been pressed as these also generate an interrupt as described in section 6.2.4.

To read the tacho count the host sends the ASCII character ' $\mathrm{k}$ ' to the battery monitor. On receiving this command the microcontroller sends back the contents of the three tacho registers. 


\subsubsection{Data Transfer Commands}

The data transfer commands allow data to be written to and read from the $\mathrm{E}^{2} \mathrm{PROM}$ on the battery monitor system board. These commands are summarised in table 6.2.

\begin{tabular}{|c|c|c|c|c|}
\hline Command & Character & Parameters & Returns & Description \\
\hline $\begin{array}{l}\text { Read } \\
\text { E2PROM }\end{array}$ & $r$ & $\begin{array}{l}<\text { high addr } \\
\text { byte }><\text { low } \\
\text { addr byte }>\end{array}$ & $\begin{array}{l}\text { Byte at address } \\
\text { location }\end{array}$ & $\begin{array}{l}\text { Read data from E2PROM located at the address } \\
\text { specified by <high addr byte }><\text { low addr byte>. }\end{array}$ \\
\hline $\begin{array}{l}\text { Write } \\
\text { E}^{2} \text { PROM }\end{array}$ & $w$ & $\begin{array}{l}<\text { high addr } \\
\text { byte }><\text { low } \\
\text { addr byte }> \\
<\text { data byte> }\end{array}$ & * & $\begin{array}{l}\text { Writes data contained in }<\text { data byte }>\text { parameter to } \\
E^{2} \text { PROM location specified by }<\text { high addr byte }><\text { low } \\
\text { addr byte }>\text { parameters. }\end{array}$ \\
\hline
\end{tabular}

Table 6.2: $\quad$ Summary of the battery monitor data transfer commands.

Since the $E^{2} P R O M$ used can store $8 \mathrm{k}$ of data 13 address bits are required. These are contained within a 16 bit address made up of two 8 bit bytes, the high address byte and the low address byte. To initiate a read from the $E^{2} P R O M$ the host sends an ' $r$ ' character followed by the high and low address bytes containing the E'PROM address to be read from. On receiving this command the microcontroller sends a READ instruction to the $E^{2} \mathrm{PROM}$ followed by the 16 bit address. The $\mathrm{E}^{2} \mathrm{PROM}$ responds by sending back the data byte located at that address. On receiving the data byte the microcontroller sends that byte back to the host.

To write to an $E^{2} P R O M$ address the host sends a ' $w$ ' character to the battery monitor followed by the high and low address bytes specifying the location to be written to and the data byte to be written. After receiving this data the microcontroller must first set the $E^{2}$ PROM's write enable latch. This is done by sending the WREN command to the $E^{2}$ PROM. The microcontroller then sends a WRITE command to the E'PROM followed by the 16 bit address to be written to and the data byte to be written. On completion of the write command the microcontroller send an '*' character back to the host to indicate that the write has been completed.

\subsubsection{Display Commands}

There are three commands that are associated with the battery monitor displays. These are summarised in table 6.3. These commands do not return any values to the host. 


\begin{tabular}{|l|l|l|l|}
\hline Command & Character & Parameters & Description \\
\hline $\begin{array}{l}\text { Update } \\
\text { LCD display }\end{array}$ & $\mathrm{f}$ & $\begin{array}{l}<\text { line \#> (0..3) } \\
<\text { text> }\end{array}$ & $\begin{array}{l}\text { Writes the text string in <text> to the LCD line specified by <line \#> } \\
\text { parameters. }\end{array}$ \\
\hline $\begin{array}{l}\text { Update } \\
\text { LED display }\end{array}$ & $\mathrm{f}$ & $\begin{array}{l}<\text { gauge level> } \\
(0.10)\end{array}$ & $\begin{array}{l}\text { Updates the LED 'fuel' gauge display with the level specified in <gauge } \\
\text { level>. }\end{array}$ \\
\hline $\begin{array}{l}\text { Set LED } \\
\text { brightness }\end{array}$ & $\mathrm{b}$ & $\begin{array}{l}<\text { brightness> } \\
(0 . .15)\end{array}$ & $\begin{array}{l}\text { Sets the LED display brightness specified in the <brightness> } \\
\text { parameter. }\end{array}$ \\
\hline
\end{tabular}

Table 6.3: $\quad$ Summary of the battery monitor display commands.

The first command is used to update the LCD panel. Each of the four lines of LCD panel can be updated individually. An ' 1 ' character is sent to the battery monitor followed by the line number to be updated and a text string containing the 20 characters to be displayed on that line. If the text string to be displayed contains less than 20 characters the text string must be padded to 20 characters with spaces.

On receiving the LCD update command the microcontroller sends a command to the LCD panel to position the LCD cursor at the start of the specified line. The microcontroller then sends the characters contained within the text string to the LCD panel one at a time as they are received from the host. After 20 characters are received the microcontroller returns to get the next command.

There are two commands associated with the LED state of charge meter. The first command is used to update the display and the second command is used to adjust the brightness of the display. To update the display an ' $\mathrm{f}$ ' character is sent to the battery monitor followed by a byte containing the number of LEDs between 0 and 10 to be displayed. The display is designed to change colour when the state of charge falls to $40 \%$ or below. To do this the microcontroller must first determine if four or less LED are to be turned on indicating that the state of charge is below $40 \%$. Two lookup tables are then used to find the correct bytes to send to the display driver to turn on the correct LEDs. One lookup table is used if four or less LEDs are to be turned on and the other is used if five or more are to be turned on.

The LED display brightness can be changed by sending a ' $b$ ' character and a byte containing the display brightness level to the battery monitor. The display brightness level value can be set between 0 and 15 corresponding to the minimum and maximum display brightness levels. This value is sent to the LED display driver which sets the duty cycle of the display and hence the brightness. 


\subsubsection{Control Commands}

The control commands are used to read back status information from the battery monitor and to power down the battery monitor. The four commands are summarised in table 6.4. No additional parameters are used with these commands.

\begin{tabular}{|c|c|c|c|}
\hline Command & Character & Returns & Description \\
\hline $\begin{array}{l}\text { Pushbutton } \\
\text { read }\end{array}$ & $i$ & $\begin{array}{l}\text { LCD screen \#+ } \\
\text { rest button } \\
\text { status }\left({ }^{\prime} r \text { ' } / n^{\prime}\right)\end{array}$ & $\begin{array}{l}\text { Reads LCD screen number set by } A \& \text { pushbuttons and status of } \\
\text { the trip reset button and sends that information back to the host. }\end{array}$ \\
\hline $\begin{array}{l}\text { Monitor } \\
\text { power } \\
\text { status }\end{array}$ & $\mathrm{p}$ & $\begin{array}{l}\text { Power status } \\
\text { byte ('b'/'c'/'m') }\end{array}$ & $\begin{array}{l}\text { Returns an byte corresponding to the state of the power supply to the } \\
\text { monitor - backup battery ('b'), charger supply ('c') or mains supply } \\
\left({ }^{\prime} \mathrm{m}\right) \text {. }\end{array}$ \\
\hline $\begin{array}{l}\text { Headlight } \\
\text { status }\end{array}$ & $\mathrm{h}$ & $\begin{array}{l}\text { Headlight } \\
\text { status (' } 1 \text { '/ } 0 \text { ') }\end{array}$ & Returns ' 1 ' if headlights are on and ' 0 ' if they are off \\
\hline Shutdown & s & - & $\begin{array}{l}\text { Shutdown the monitor power supplies and places the micro into its } \\
\text { sleep mode. }\end{array}$ \\
\hline
\end{tabular}

Table 6.4: Summary of the battery monitor control commands.

The pushbutton read command is used to read back the status of the three pushbuttons on the front panel of the monitor. These buttons allow up and down scrolling through the LCD screens and the trip meter to be reset. Because the pushbutton status is polled by the host it is possible that if multiple screens were scrolled through between polling intervals, some of the pushbutton presses would get lost. To overcome this problem the microcontroller acknowledges presses of the up and down buttons when they occur and increments or decrements a register containing the number of the screen to be shown on the LCD.

The pushbuttons are connected via debounce circuits to pins on port $B$ of the microcontroller. Whenever a pushbutton is pressed or released a change of logic level occurs on those pins and an interrupt is generated. This causes the microcontroller to branch to the interrupt service routine which first checks which interrupt has occurred. If an interrupt has been generated from a logic level change on port $B$ the input pins are checked to see which pushbutton has been pressed. If it is the up or down buttons the screen register is either incremented or decremented. As an interrupt is generated on the release of a pushbutton also, only one increment or decrement occurs for every two interrupts. If the trip meter reset button was pressed then the button's status is latched until the pushbutton read command is received from the host. On receiving this command the screen number and the status of the reset button is send back to the host. The screen number can then be used by the host to determine what information should be displayed on that particular screen and the reset button status used to initiate a trip counter reset if the reset button has been pressed.

The power status of the battery monitor can also be read back from the monitor. On issuing this command the battery monitor checks the system power and charger power 
logic signals from the power supply. From these two signals the microcontroller determines whether the battery monitor is being powered from the main supply, the charger supply or, if both signals are low, the backup supply. A ' $\mathrm{m}$ ', a ' $c$ ' or a ' $\mathrm{b}$ ' are returned to the host to indicate the respective supply that is operating (see table 6.4). This information can be used by the host software to determine whether the car is switched on or off and whether it is being charged.

To determine whether the headlights are switched on or off the headlight status command is used. This command causes the microcontroller to check the logic signal derived from the headlights. If the headlights are on, a ' 1 ' is sent back to the host, otherwise a ' 0 ' is returned.

The final control command is the shutdown command. On receiving this command the battery monitor sends a power down command to the RTC, switches off the relay supplying power to the BMMs, the LCD display and the LED display. All of the microcontroller's I/O lines are set low to prevent the devices connected to these lines back feeding power from these lines back to the power supply lines. Also on shutdown all interrupts except for the port B logic level change interrupt are disabled. The sleep instruction is then used to place the microcontroller into sleep mode.

The system power logic signal is connected to one of the port B pins which generates an interrupt when a logic level change occurs. This is used to wake the microcontroller from sleep when the battery monitor is turned back on. This interrupt is shared with the pushbuttons. This does not cause a problem since when the main power supply is off, the pushbutton debounce circuits do not operate. The pushbutton lines therefore cannot change logic level. After waking up, the microcontroller calls the initialisation code to reinitialise the microcontroller registers and the battery monitor peripherals.

\subsubsection{Miscellaneous Commands}

The remaining commands are summarised in table 6.5 and are used to test the battery monitor communications, get the version information string from the E 2 PROM and communicate with the RTC. 


\begin{tabular}{|c|c|c|c|c|}
\hline Command & Character & Parameters & Returns & Description \\
\hline Test & $t$ & - & * & $\begin{array}{l}\text { Returns '*' to show that monitor is responding to } \\
\text { commands from the host. }\end{array}$ \\
\hline Version & $v$ & - & $\begin{array}{l}\text { Version Info } \\
\text { String }\end{array}$ & $\begin{array}{l}\text { Gets the version information string stored in the } \\
E^{2} P R O M \text { and sends it to the host. }\end{array}$ \\
\hline Read time & $\mathrm{c}$ & - & $\begin{array}{l}\text { time string } \\
\text { (HH:MM:SS) }\end{array}$ & Reads the time from the RTC and sends to host. \\
\hline Read date & $d$ & - & $\begin{array}{l}\text { date string } \\
\text { (DD:MM:YY) }\end{array}$ & Read the date from the RTC and sends to host. \\
\hline Set time & $\mathrm{a}$ & $\begin{array}{l}<Y Y M M D D> \\
<\text { HHMMSS> }\end{array}$ & * & $\begin{array}{l}\text { Sends the date and time specified in the <YYMMDD }> \\
<\text { HHMMSS }>\text { parameter to the RTC. }\end{array}$ \\
\hline
\end{tabular}

Table 6.5: Summary of the remaining battery monitor commands.

The test command is used by the host to see if the battery monitor is functioning. The battery monitor sends back an '*' character to indicate it is responding to commands from the host.

The version command allows the host to read the version string contained within the monitor's E'PROM. The microcontroller on receiving this command reads each character of the version string out of the $E^{2} P R O M$ one at a time and sends each to the host until the end of the string is reached. This string can also be read by using the E ${ }^{2} P R O M$ read command with the address of the characters contained within the version string. This however requires the host to issue one command for each character in the string.

Three commands are used to set and read the date and time from the RTC. The read time and date commands are used to read the RTC time and date respectively. After receiving either of these commands the microcontroller reads the appropriate registers within the RTC and converts the four bit BCD values stored in the RTC registers to ASCII characters. The date or time values are formatted into standard date or time strings and sent to the host.

To set the RTC time and data the set time command is issued from the host followed by the date and time strings. The numbers contained within the data and time strings are converted from ASCII values to four bit BCD numbers and are sent to the RTC date and time registers. On completion of this command an '*' character is sent back to the host to signal that the command has been successfully completed. 


\subsection{Host Software}

The host software was written in Visual Basic 4 and runs under Windows 95/NT. Visual Basic uses forms that are drawn up and become the windows and dialog boxes in the software. The objects on those forms each have events associated with them such as when a button is clicked. Code can be attached to each of these events and is executed when that event occurs. Due to the event driven nature of the programming language the program flow is not always sequential and several events may be serviced at the same time.

The battery monitor host software is required to perform the control and calculation requirements of the battery monitor. In addition to these requirements it is also required to provide data logging to allow battery monitor data to be saved and analysed at a later date.

Two versions of the host software were written. The first was written to only perform battery monitoring on a single battery. This was used for bench testing of the monitor using a single battery. This software was later upgraded to form the second version of the software that was able to monitor multiple batteries in the electric car. A copy of both versions of the software have been included in Appendix $\mathrm{E}$ along with the source code.

\subsection{Single Battery Test Version}

Figure 6.2 shows the main window of the single battery version of the monitor software. This window shows the data being received from the battery monitor, the status of the battery and the results of the coulometric and open circuit voltage calculations.

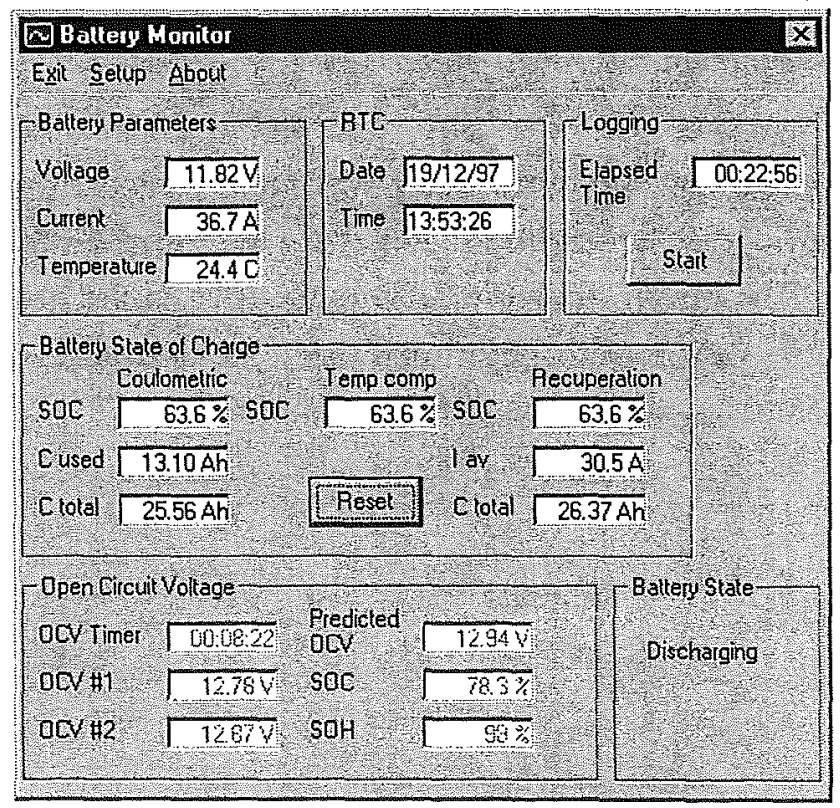

Figure 6.2: The main window of the battery monitor single battery test version. 
The data displayed in the window is grouped using frames. Within these frames the actual data is displayed in text boxes. At the top of the window the three measured battery parameters - voltage, current, and temperature, are shown. Next to this the date and time from the real time clock are shown as well as the logging elapsed time and the logging start/stop button. Below this the three battery state of charge calculations obtained from coulometric calculations are displayed. The state of charge obtained with and without compensation for temperature and recuperation effects are shown to allow comparisons to be made between the compensated and uncompensated calculations. In addition to the state of charge the total calculated battery capacity $\left(C_{\text {total }}\right)$, the capacity consumed from the battery $\left(C_{u s e d}\right)$ and the average battery current $\left(I_{a v}\right)$ are also displayed. A reset button is also included to allow the state of charge to be manually reset to $100 \%$.

At the bottom of the main window the calculation parameters and results associated with the open circuit voltage calculations are shown. The left three text boxes show the elapsed time since the battery has finished discharging and the two open circuit voltage measurements taken. On the right the calculated open circuit voltage prediction is displayed along with the state of charge calculated from this value and the estimated state of health of the battery. All of the open circuit voltage text boxes are greyed out when discharging or charging begins again indicating that the data has become invalid.

The remaining frame shows the status of the battery. The text in this frame shows charging, discharging, or idle, depending on the battery state.

The main window contains three menus which give options to exit from the program, set the calculation and logging parameters, and bring up an 'about' dialog box. The exit menu does not contain any submenus and runs the monitor shutdown sequence before exiting the program. The second menu contains two options that bring up separate dialog boxes to configure the monitor parameters and to control the data logging function. The battery monitor configuration dialog box is shown in figure 6.3 and allows parameters for the BMM channels, the coulometric and open circuit voltage calculations, the tacho and the LED display brightness levels to be set. 


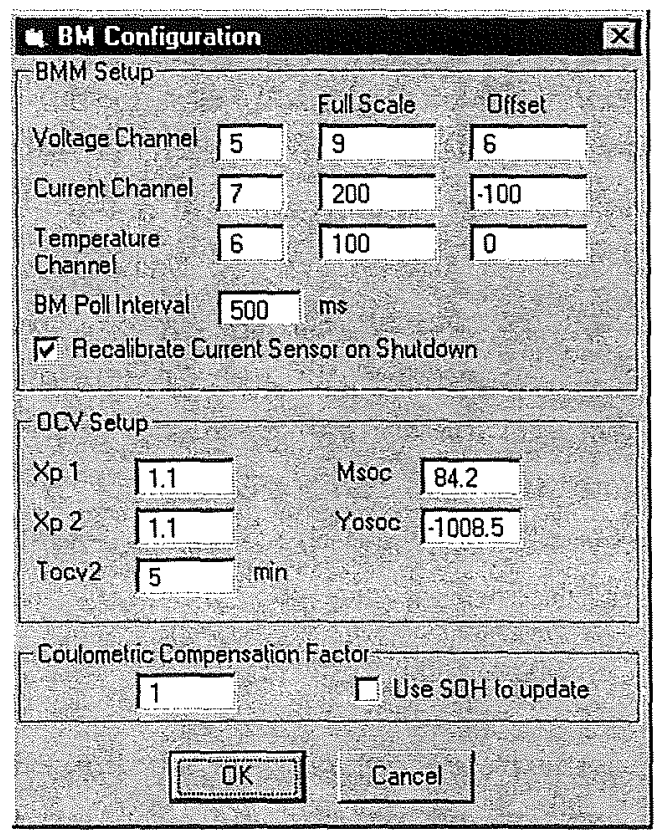

Figure 6.3: The battery monitor configuration dialog box.

The BMM parameters can be set to allow the channel that each of the three battery measurements should be made from to be specified, as well as the full scale and offset for each channel. A further parameter sets the interval at which the BMM is polled. A check box is also available which gives the option to recalibrate the current sensor when the monitor is shutdown. This option is used to automatically correct for the small offset drift of the current sensor. If this option is selected the sensor offset is stored when monitor is shutdown and no current is flowing though the sensor. The stored value is used to offset future current measurements.

The configuration dialog box also allows the parameters for $X p$, Toc (the time to wait before the second open circuit voltage measurement should be made) and the slope and intercept values that relate the open circuit voltage to the state of charge, Msoc and Yosoc. The coulometric compensation factor used to compensate the coulometric measurements as the batteries age can also be set here. An option is also given to automatically update this value whenever a battery state of health prediction is made. 


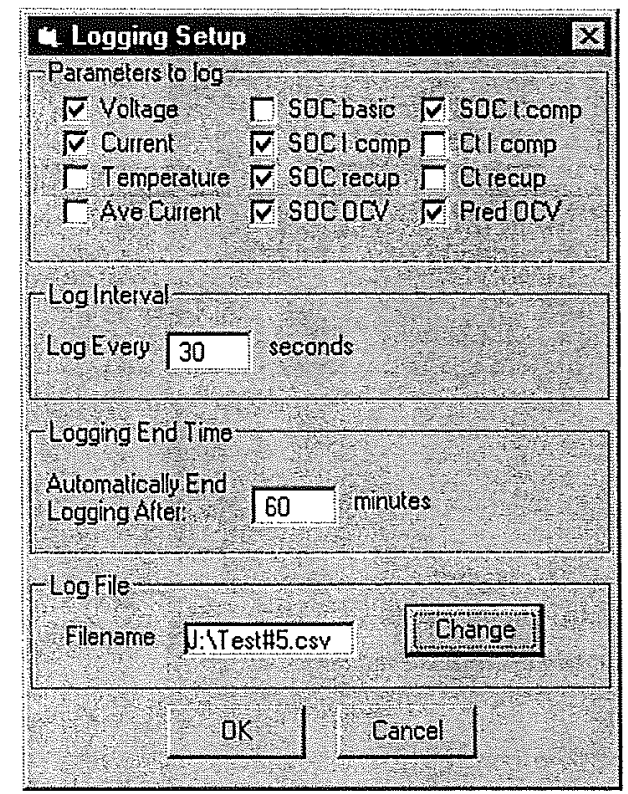

Figure 6.4: The logging setup dialog box.

Figure 6.4 shows the logging setup dialog box. This dialog box has a series of check boxes which are used to select the parameters to be logged. The interval at which data should be logged can be specified in seconds as well as the filename the data should be stored using. A button is provided to change the filename using a standard Windows file save dialog box. The logged data is stored in a comma delimited CSV data formatted which can be easily imported into a spreadsheet for analysis of the data. The final parameter in this dialog box allows a time in minutes when the logging should be ended to be set. If this parameter is not specified, logging will continue until it is stopped manually by the user.

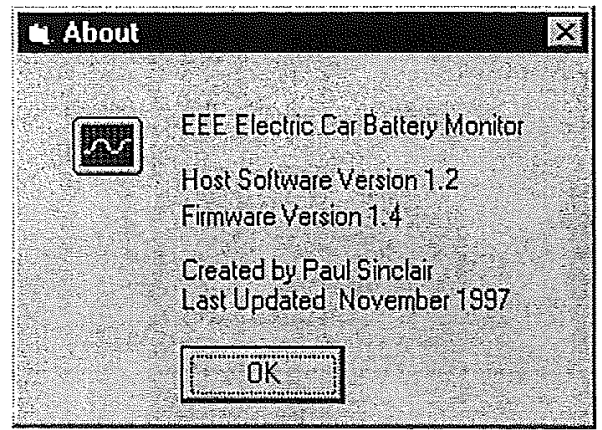

Figure 6.5: The 'about' dialog box.

The 'about' dialog box brought up when the 'about' menu is clicked is shown in figure 6.5. This dialog box displays information about the software, including the version numbers of both the host and battery monitor software. 


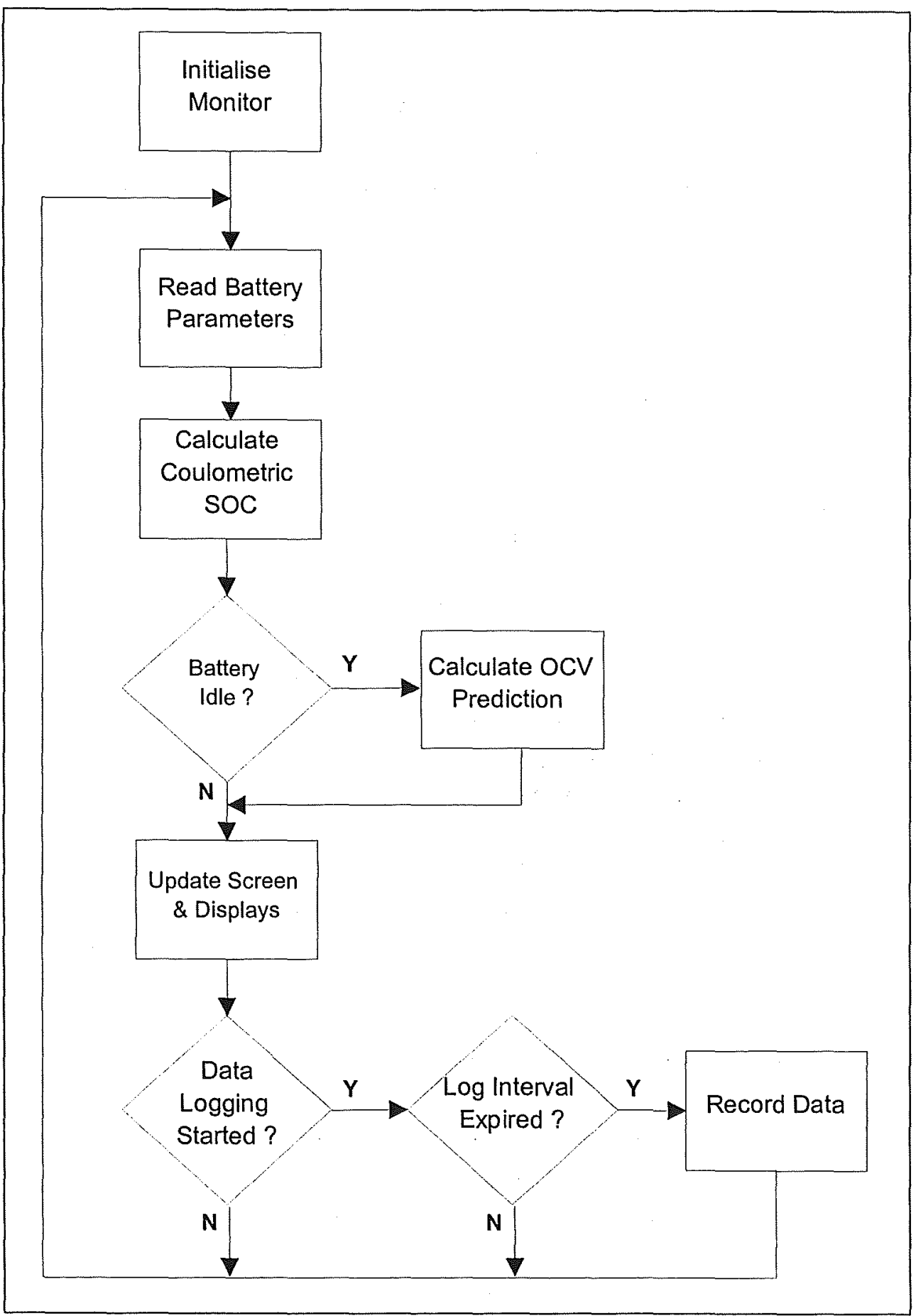

Figure 6.6: The program flow of the host software.

Figure 6.6 shows the basic program flow of the host software. It should be noted that since software written in Visual Basic is event driven, the flow may not exactly follow that shown in figure 6.6. Also other processes may occur in parallel to the main program flow when other events are triggered such as menu options being clicked. 


\subsubsection{Initialisation}

When the host software is started it first prompts the user for the RS-232 port that the battery monitor is attached to. On receiving this information the software polls the battery monitor using the monitor's test command to see if it is responding. If no response is received the user is prompted with a 'Battery monitor not found' error with the options to either retry or cancel. If cancel is selected the program exits.

All communications from the host software are handled by the Visual Basic communications control. This provides a set of objects that are used to control and transfer information from the computer's comm ports.

Once communications have been established with the battery monitor the monitor configuration parameters and values stored from the last time the monitor was used are loaded. These values are stored in the battery monitor's $E^{2} \mathrm{PROM}$. This approach means that these values do not need to be stored on the host computer so the monitor can be run from any computer without having to set up these values first.

On completion of loading these values the timer event that controls the main program loop execution is initialised and started.

\subsubsection{Main Program}

The main software loop, shown in figure 6.6, is executed each time the timer event occurs. The timer event interval is set by the BMM poll interval set in the battery monitor configuration dialog box (figure 6.3). Each time the timer event occurs the battery parameters are read back from the monitor and the various calculations are performed on the data obtained. The results from the calculations are displayed both on the screen of the host computer and on the battery monitor displays. This data is also logged if data logging has been started. If the battery is in an idle state, the open circuit voltage calculations are made.

\subsubsection{Coulometric Calculations}

The state of charge based on the coulometric method is calculated first. This calculation is made in several stages. First the amount of capacity used $\left(C_{u i}\right)$ since the battery data was last measured is calculated. $C_{\mathcal{U}}$ is calculated assuming that the current is constant during the measuring interval by using a linear integration method (equation 6.1). 


$$
C_{u i}=I \cdot \frac{t_{i}}{3600}
$$

The time interval $\left(t_{i}\right)$ is the time interval between this measurement and the last. This measuring interval is controlled by the timer event which is set to occur at regular set intervals. It cannot however be guaranteed that the timer event will occur at exactly this time due to other processes which may be occurring at the same time both within the host software and other programs which may be running at the same time within the Windows environment. It is therefore necessary to derive this value from the actual time that has elapsed since the last timer event occurred. This is calculated from the system time each time the timer event occurs. $t_{i}$ is in seconds so it must be converted to hours to give a result in ampere-hours hence the division by a factor of 3600 in equation 6.1

The percentage of the total battery capacity used in the measuring time interval is calculated next. This is done by dividing the capacity used by the calculated total capacity of the battery (equation 6.2). The result is multiplied by 100 to convert it to percent.

$$
S O C_{i}=\frac{C_{u i}}{C_{t i}} \times 100
$$

The total capacity $\left(C_{t i}\right)$ used in equation 6.2 is calculated in each time interval using equation 6.3. It gives the total battery capacity that is available under the current battery conditions. $C_{t i}$ is calculated using the Peukert equation (equation 3.1) using constants derived for manufacturer's data for these particular batteries. The result is multiplied by a temperature compensation factor $\left(t_{c}\right)$ to account for the changing performance with temperature and by a compensation factor $(E)$. E takes into account the errors that occur in the coulometric calculations as the battery ages. This value can either be manually entered in the battery monitor configuration dialog box or can be derived from the open circuit voltage state of health calculations if that option is selected.

$$
C_{t i}=41 \cdot I^{-0.17} \cdot T_{c} \cdot E
$$

The temperature compensation factor $\left(T_{c}\right)$ is calculated using equation 6.4. The temperature compensation is calculated using a linear approximation of the temperature characteristics in figure 3.3. Compensation for temperature is only calculated when the battery temperature is below $12.5^{\circ} \mathrm{C}$ and the current is below $10 \mathrm{~A}$ as temperature compensation is unnecessary outside of these conditions. When temperature compensation is not necessary $T_{\mathcal{C}}$ is set to 1 .

$$
T_{c}=(0.008 . T+0.9)
$$

The total battery capacity calculation (equation 6.3) is used only during discharge since it does not hold during charge. The nominal battery capacity of $36 \mathrm{Ah}$ is used when charging 
is taking place since experimental results have shown that under the charging conditions present in the car the charge acceptance is very close to $100 \%$.

Finally the battery state of charge is calculated using equation 6.5 by subtracting the percentage of charge used in the measuring interval $\left(S O C_{i}\right)$ from the battery state of charge $\left(S O C_{C}\right)$. The total state of charge may be manually reset back to $100 \%$ by clicking the reset button in the main window.

$$
S O C_{C}=S O C_{C}-S O C_{i}
$$

In order to calculate the state of charge so that it takes into account recuperation effects the average current $\left(I_{a v e}\right)$ is used in the total capacity calculations (equation 6.3). Iave is the average current throughout the discharge and is calculated using equation 6.6. It is calculated by dividing the total battery capacity $\left(C_{u}\right)$ used up to that point by the total discharge time ( $t$ discharge).

$$
I_{\text {ave }}=\frac{C_{u}}{t_{\text {discharge }}}
$$

\subsubsection{Open Circuit Voltage Calculations}

The open circuit voltage (OCV) calculations are mostly performed by a separate subroutine. The flow of this subroutine is shown in the flow diagram of figure 6.7.

The open circuit voltage calculations can only be made when the battery is not being charged or discharged. The battery state is therefore tested and if the battery is in an idle state a timer is started and several control flags are set. If the battery enters a discharge or charge state before the OCV calculation is complete the OCV calculation is terminated until the battery again enters an idle state.

Each time the OCV subroutine is called the OCV elapsed time is updated from the system time and is checked to see if either of the two measurement times have elapsed. The first open circuit voltage measurement $\left(V_{O c 1}\right)$ is made after one minute has expired. The second measurement $\left(V_{O C 2}\right)$ is made after the time $t_{o c}$ set in the monitor configuration dialog box (figure 6.3) expires. Once this measurement is made the OCV calculations are performed.

The OCV recovery slope $(M)$ is calculated first using equation 6.7. $t_{1}$ and $t_{2}$ are time times that the actual measurements of $V_{o c 1}$ and $V_{o c 2}$ are made.

$$
M=\frac{V_{o c 2}-V_{o c 1}}{\log _{10}\left(t_{2} / 60\right)-\log _{10}\left(t_{1} / 60\right)}
$$




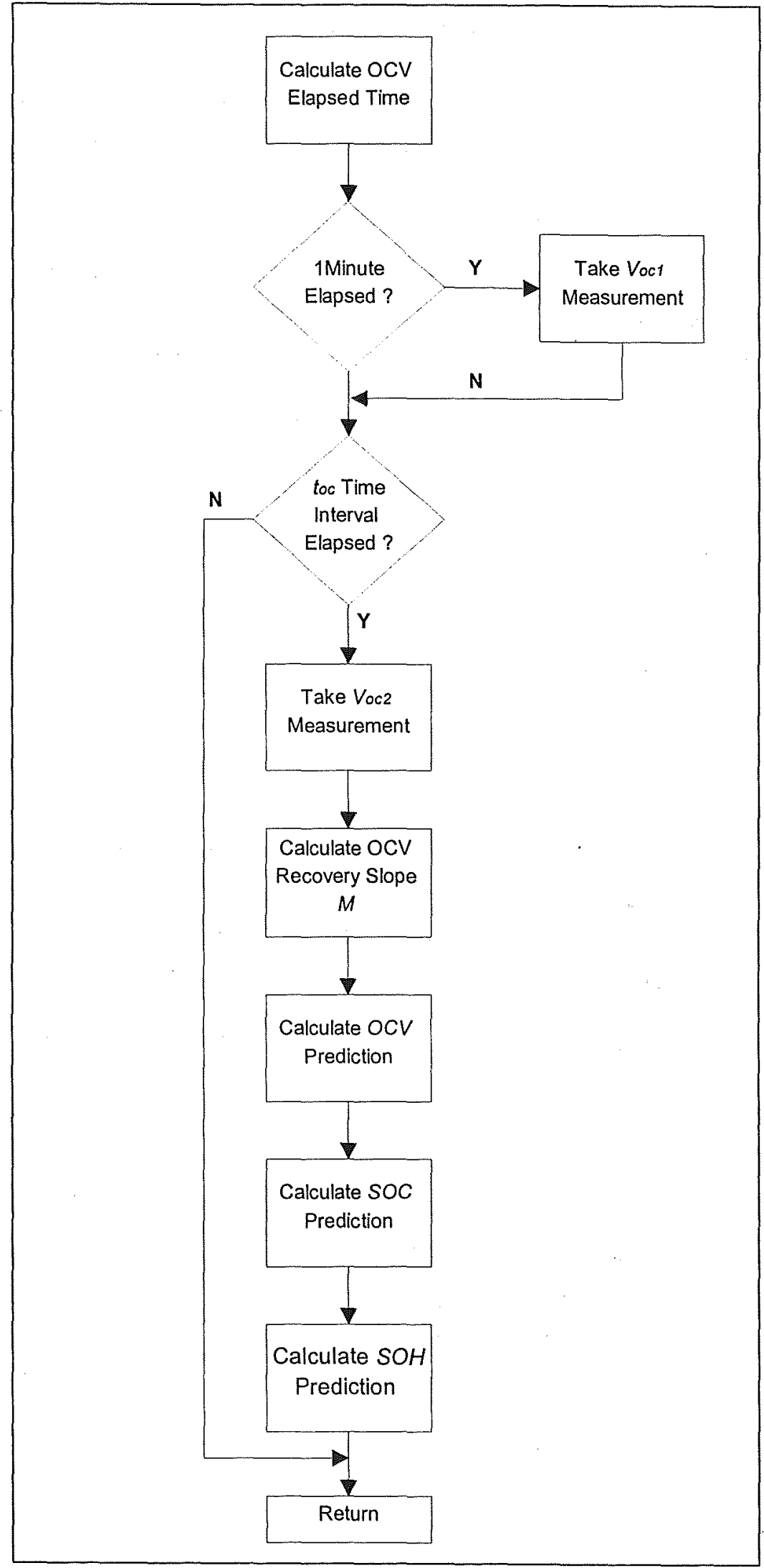

Figure 6.7: The program flow of the OCV calculation subroutine in the host software. 
Equation 6.8 has an extra term that is not present in equation 4.7. This term is the $\log _{10}\left(t_{1} / 60\right)$ term which since $t_{1}$ is one minute would normally be zero. It cannot always be guaranteed that the first measurement will be made after exactly one minute due to software processing delays so the equation uses the actually time at which $V_{o c 1}$ was made $\left(t_{1}\right)$. This prevents errors in the calculation of $M$ occurring.

$$
V_{o c}=M \cdot X p+V_{o c 1}
$$

Once the slope of the recovery curve has been determined equation 6.8 is used to predict the fully recovered open circuit voltage $\left(V_{o c}\right)$. The value of the asymptote intersection point $\left(X_{p}\right)$ is set in the monitor configuration dialog box (figure 6.3). Two values can be set for $X_{p}$. The one used depends on the coulometric state of charge. This is done since the open circuit recovery tends to change slightly at lower states of charge. A state of charge prediction can then be made from the $V O C$ using equation 6.9. The parameters $M_{S O C}$ and $Y_{O S O C}$ are derived from the state of charge to OCV characteristic shown in figure 3.4 and are also set in the monitor configuration dialog box.

$$
S O C_{o c v}=M_{s o c} \cdot V_{o c}+Y_{o s o c}
$$

The final calculation made in the OCV subroutine is the battery's state of health (SOH). This calculation is made by calculating the ratio between the state of charge from the OCV calculations $\left(S O C_{\mathrm{OCV}}\right)$ and the state of charge calculated from the coulometric calculations $\left(S O C_{C}\right)$ using equation 6.10. Since the coulometric state of charge calculation is compensated by the SOC calculation factor $E$ (equation 6.3) this factor must be removed hence this factor also appears in this equation.

$$
S O H=\frac{S O C_{o c v} \cdot E}{S O C_{C}} \times 100
$$

\subsubsection{Data Logging}

When the logging start button is clicked the filename specified in the logging configuration dialog box is opened (figure 6.4). If a filename has not been specified the logging configuration dialog box is displayed to allow the user to enter one. A header is written to the log file to identify the file, record the start date and time of the log file, and list the parameters that have been selected to be logged. A flag is also set to indicate that logging is enabled and to tell the main program to execute the data logging routines. When logging is started the start button becomes the logging stop button. This is achieved by changing the button's caption and testing to see if logging has been started when it is next clicked.

Once the data logging has been started the logging code is executed when each timer event occurs. This code calculates the elapsed time since logging was started for display 
on the screen and also checks to see if the logging interval has expired. If it has, the data that has been selected to be logged is written to the log file, along with the elapsed time in hours. The elapsed time is also compared to the logging end time if this has been set. If this time has been reached the logging is terminated.

\subsubsection{Shutdown Sequence}

The shutdown sequence is initiated by either clicking on the exit menu or clicking the close button in the top right hand corner of the window. The shutdown routine first checks to see if logging is in progress. If it is the user is prompted to make sure they do indeed wish to quit. If they do the logging file is closed and the shutdown sequence is run, otherwise they are returned to the main window and the program continues. The shutdown sequence starts by disabling the timer event to stop execution of the main program loop. A "Shutting down monitor" message is displayed on the monitor's LCD screen. If the recalibrate current sensor option has been selected it is assumed that zero current is flowing though the current sensor so any reading from the current sensor is it's offset. This value is measured and stored to correct the measurements next time the monitor is used.

The next part of the shutdown sequence involves writing the battery monitor values and parameters back to the monitor's $E^{2} P R O M$ so that they can be reloaded when the monitor is powered up again. These values include the battery state of charge and state of health, the trip meter and distance gone measurements and various other values used in the calculations. The battery monitor configuration parameters are also written to the $E^{2} P R O M$ if they have changed from the parameters that were loaded when the program was started. Once this has been completed a shutdown command is sent to the battery monitor to power it down, the computer's RS-232 port used for communication is closed and the program terminates.

\subsection{Multiple Battery Test Software}

The second version of the host software was written to perform measurements on up to twenty batteries. It was designed to be used on a laptop computer that could be used in the electric car while it was running. This version is very similar to the single battery test software. Additional code was written to perform the OCV on up to twenty batteries rather than a single one, calculate the car's tripmeter and distance remaining to travel predictions and control display dimming when the headlights are turned on.

Figure 6.8 shows the main window of the software. Since this version of the software is designed for use in the car, the LCD display on the dash is used as the main display for data from the monitor. The main window of the software duplicates much of this information and also includes some additional information that is useful for diagnostic purposes. The information is presented on multiple 'screens' similar to the way that 
information is displayed on the LCD display. Up and down buttons are used to move through the various 'screens.' Status information about the present battery state and the power supply of the monitor are displayed, along with the data logging controls, in the area below this.

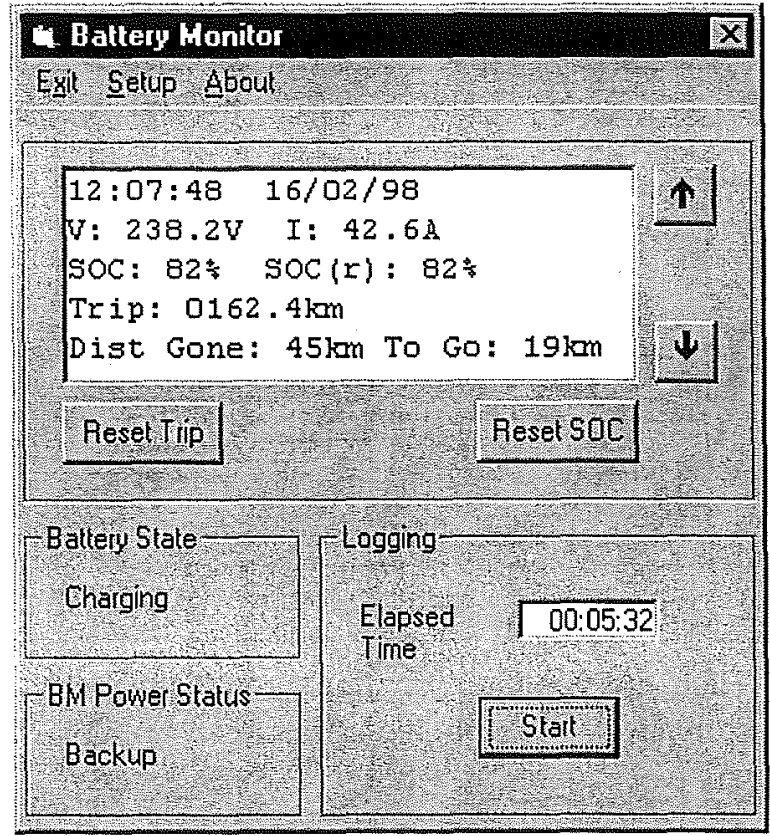

Figure 6.8: The main window of the multiple battery host software.

The configuration dialog box is shown in figure 6.9 and allows the same calculation parameters to be set as the single battery version does. Additional parameters can also be set. The first of these is the tachometer scaling factor which is used in the distance calculations in section 6.5.2. This parameter sets the number of pulses that are received from the tachometer per $\mathrm{km}$ travelled. The second set of parameters are the LED display brightness levels. Two brightness levels are used, one for daytime use and the other for nightime driving conditions.

This version allows the configuration for each of the three BMMs to be set as determined by the jumper settings on each of the BMM boards. The monitor can be configured to run with only one or two BMMs connected rather than all three. This allows less than twenty batteries to be monitored if necessary. The channel configuration for each BMM is stored internally in the software so the user does not have to specify the channel numbers for each measurement channel. As with the single battery version, scaling and offset values for each of the three types of measurement channels can be set. 


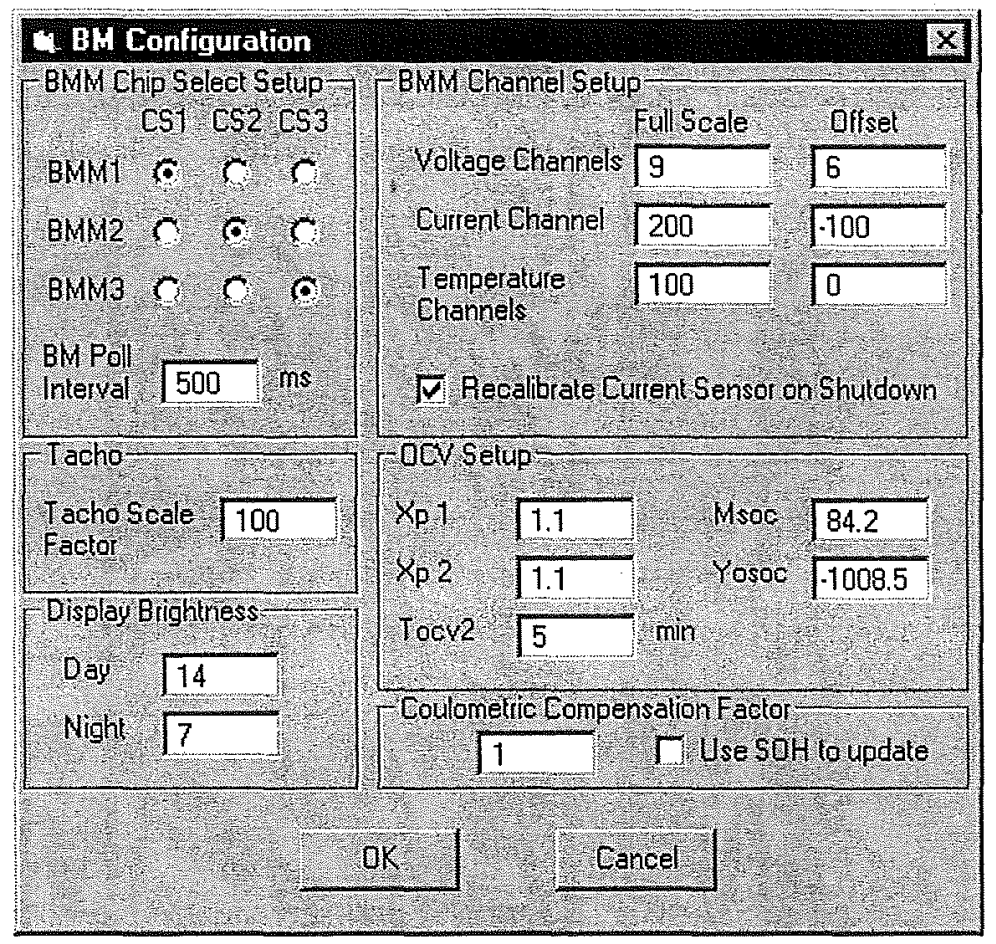

Figure 6.9: The battery monitor configuration dialog box for the multiple battery host software.

\subsubsection{LCD Display Screens}

The seven LCD display screens used in this version are summarised in table 6.7. These screens are also used with the single battery with the exception of screens 5 and 6 . The first screen displays the time and date, the battery bus voltage and current, the battery state of charge, and the tripmeter. The second screen also displays the state of charge and the tripmeter, along with the distance gone on this charge and the remaining distance that may be travelled.

The open circuit voltage measurement information is shown on screen 3. This screen changes depending on whether an OCV calculation is in progress or not. If a calculation is taking place a "Calculating OCV ...." message is displayed with the time remaining before the OCV prediction can be made. If an OCV calculation is not in progress the last valid predicted open circuit voltage and state of charge are displayed.

Screens 4, 5 and 6 are designed to show the state of health, in percent, of each of the twenty batteries in the car. Screen 4 also displays the average state of health of the battery bank. The final screen displays miscellaneous parameters that are mainly useful for diagnostic purposes. The parameters shown are the battery temperature, the energy consumption rate and the offset drift of the current sensor. 


\begin{tabular}{|c|c|c|}
\hline $\begin{array}{l}\text { Screen } \\
\text { Number }\end{array}$ & \multicolumn{2}{|l|}{ Display Contents } \\
\hline 1 & \multicolumn{2}{|l|}{ 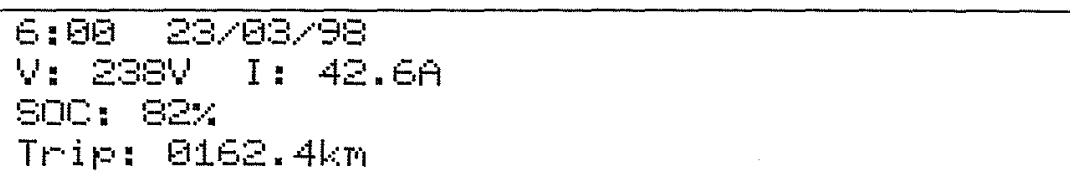 } \\
\hline 2 & \multicolumn{2}{|l|}{ 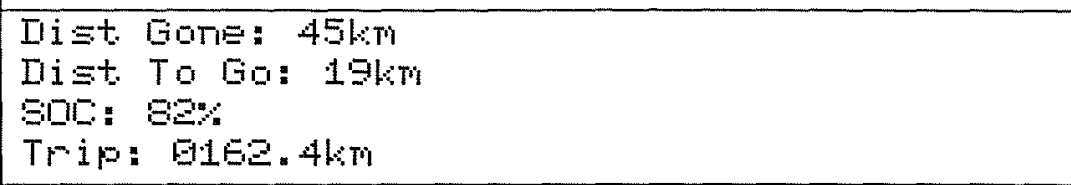 } \\
\hline 3 & 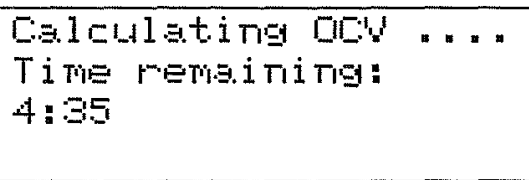 & 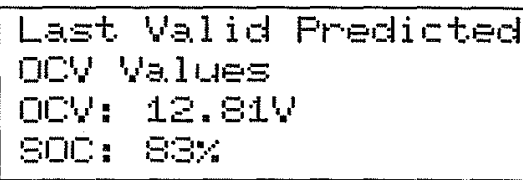 \\
\hline 4 & \multicolumn{2}{|l|}{ 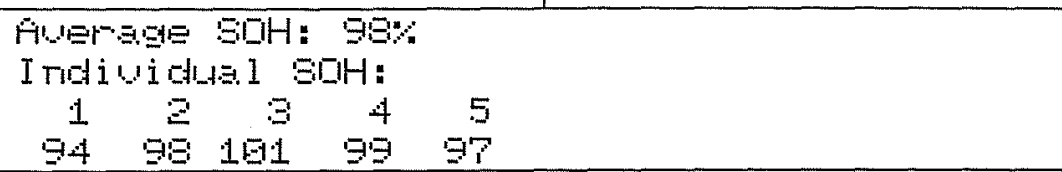 } \\
\hline 5 & $\begin{array}{rrrrr}5 & 7 & 9 & 9 & 19 \\
99 & 99 & 97 & 181 & 99 \\
11 & 12 & 19 & 14 & 15 \\
96 & 191 & 99 & 97 & 98\end{array}$ & \\
\hline 6 & $\begin{array}{ccccc}16 & 17 & 19 & 19 & 20 \\
98 & 99 & 191 & 98 & 1819\end{array}$ & \\
\hline 7 & 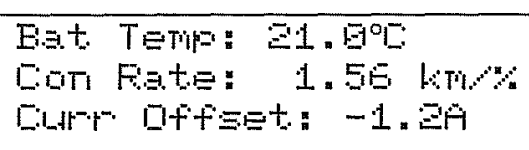 & \\
\hline
\end{tabular}

Table 6.6: $\quad$ Summary of the battery monitor LCD display screens.

\subsubsection{Distance Measurements}

The trip distance, the distance travelled since the last charge, and the remaining distance that can be travelled on the remaining charge, are calculated by a separate subroutine in this version of the software. The tacho count is read from the battery monitor and the previous tacho count is subtracted from this value to give the distance travelled in the last measurement interval. This value is divided by the tacho scale factor to give a value in kilometres and is added to the trip counter and the distance gone counter. The latter of these counters is automatically reset when the batteries are charged so it gives the distance travelled on this particular charge.

In order to predict the remaining distance that the car will travel an average consumption rate must be calculated. Equation 6.11 is used to do this and gives a consumption rate $\left(c_{R}\right)$ in $\mathrm{km} / \%$ of charge. The distance gone $\left(d_{\text {gone }}\right)$ mentioned above is used in this equation as well as the state of charge when the car is switched on $\left(\mathrm{SOC}_{o}\right)$. This calculation is only made after the car has travelled over $2 \mathrm{~km}$ and the state of charge has dropped by more than $2 \%$ to avoid false estimates being made straight after the car has 
been switched on and has not travelled any distance. Up to this point the consumption rate stored when the monitor was last shutdown is used.

$$
\begin{aligned}
& c_{R}=\frac{d_{\text {gone }}}{S O C_{0}-S O C} \\
& d_{\text {togo }}=c_{R} \times S O C
\end{aligned}
$$

The distance to go $\left(d_{\text {togo }}\right)$ is calculated by multiplying the consumption rate by the state of charge as in equation 6.12. Since the consumption rate is continuously being updated this method of calculating the remaining distance takes into account the current driving conditions and performance of the car. 


\section{Results}

\subsection{Initial Testing and Calibration}

The monitor hardware was initially tested in conjunction with the microcontroller code by manually sending commands to the monitor from a computer running a terminal program. This allowed bugs in the microcontroller code and design errors in the hardware to be found and corrected. Only one BMM was built up and used for testing purposes, capable of measuring six individual battery voltages, current and temperature.

Once the battery monitor-hardware-was found to be working correctly the input channels of the BMM were calibrated. The voltage measurement channels were calibrated using a precision voltage regulator setup to provide two switchable voltages corresponding to the upper and lower voltage limits of the input channels. This allowed the gain and offset to be set for each channel. The current channel was setup by passing known currents through the current sensor and adjusting the gain and offset of the input processing circuitry to give the correct current measurements. The remaining channel, the temperature channel, only required the channel gain to be set to give the correct readings from the pre-calibrated temperature sensor.

The host software was tested and debugged during the software development process using the previously tested hardware. The software was tested to ensure that the correct calculations were being made and also that the battery monitor control functions were correct and occurring at the correct times.

\subsection{Bench Tests}

The main objectives of the monitor bench tests were to test the functionality of the monitor algorithms as well as, where possible, the accuracy of results produced. The coulometric and OCV algorithms were tested independently over a range of battery conditions and the results compared from each. The coulometric algorithms were also tested during battery charging and low temperature discharge cycles to evaluate their performance under these conditions.

The tests were performed on a single battery, of the same type as used in the Department's electric car, and purchased specifically for the task. This battery was put though a number of discharge cycles during testing and was also used to produce the characteristics in 
chapter 3. The same system used for logging battery data (appendix A) was also used in many of the discharge tests to automatically control the discharge process.

\subsubsection{Coulometric Results}

The main aim of the first tests were to confirm that an accurate estimate of the battery state of charge was being given from the coulometric calculations. In order to do this the test battery was discharged at several different discharge rates at constant, or very close to constant, discharge currents. For each of these currents the battery was initial fully discharged to determine the total discharge time. With this information and the fact that the discharge was occurring at a constant rate, the state of charge could be accurately calculated based on the duration of the discharge. This was done by calculating the ratio of the time since the start of the discharge and the measured total discharge time. The state of charge calculated using this method could then be compared to the state of charge calculated by the battery monitor.

Discharge tests were carried out at discharge rates of $7.2 \mathrm{~A}\left(\mathrm{C}_{5}\right), 18 \mathrm{~A}\left(\mathrm{C}_{2}\right), 36 \mathrm{~A}(\mathrm{C}), 54 \mathrm{~A}$ $(1.5 \mathrm{C})$ and $72 \mathrm{~A}(2 \mathrm{C})$. Tests at the $\mathrm{C}_{5}$ rate were carried out using the constant current discharger described in section A2 of appendix A. The other discharge rates were achieved using a set of resistive load banks. These were manually adjusted to keep the discharge current very close to constant as the battery voltage gradually fell during discharge.

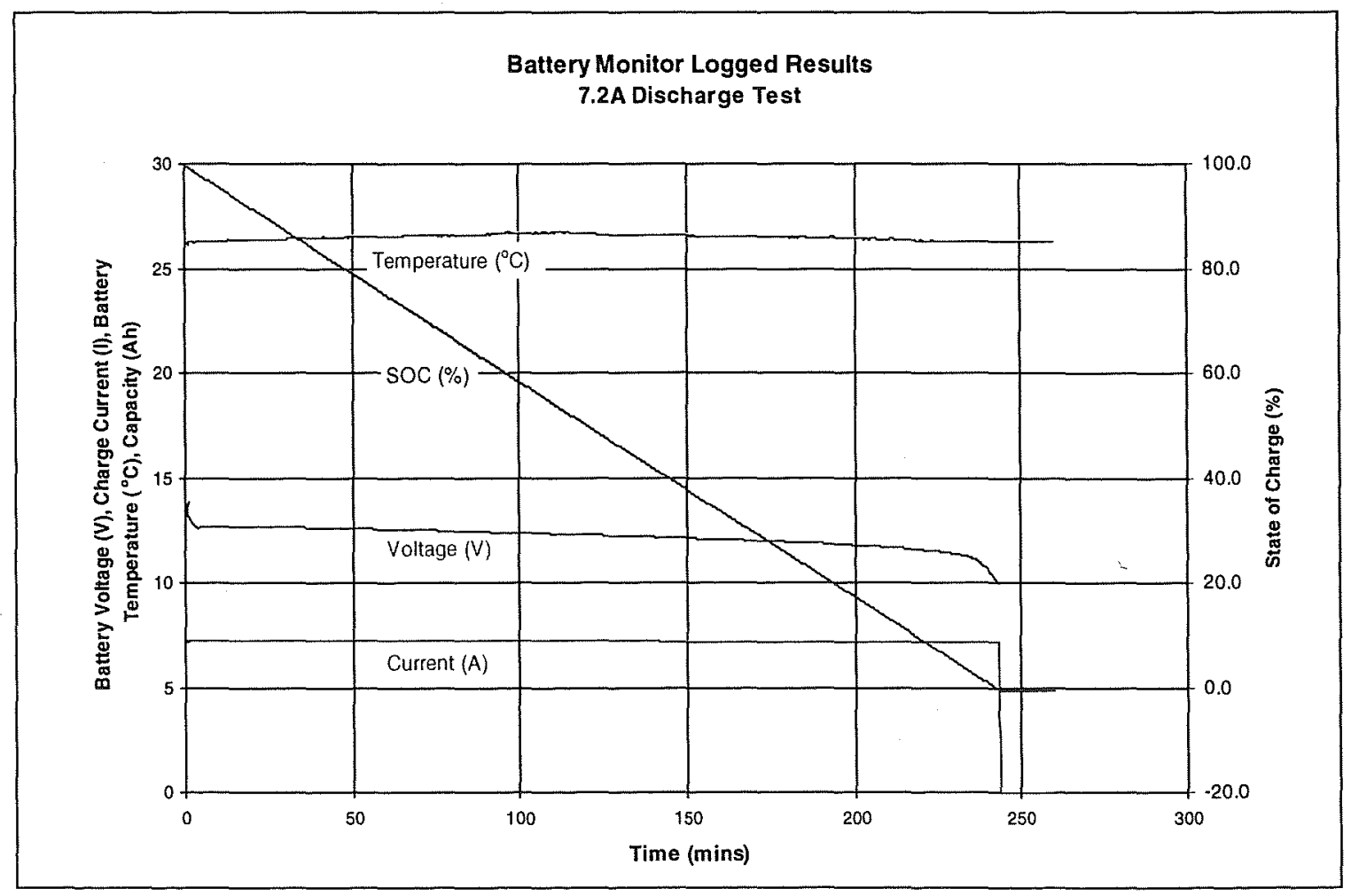

Figure 7.1: The results logged by the battery monitor during a 7.2A discharge cycle. 
The data logging function of the battery monitor was used to generate the graph in figure 7.1 during a 7.2A discharge test. The graph shows the battery voltage, current and temperature, as well as the calculated battery state of charge. The current is essentially constant until the discharge is cut off at the end of the discharge. The voltage falls very slowly over most of the discharge until a point is reached near the end of the discharge where the voltage starts to fall off at a greater rate. Since the discharge current is constant the state of charge decreases linearly until zero state of charge is reached The battery temperature rises slightly over the first two hours of the discharge and then begins to fall. The initial rise in temperature is due to internal heating in the battery while the fall is most likely due to the falling ambient temperature as this test was carried out overnight.

Table 7.1 lists state of charge measurements taken during the 7.2A discharge test shown in figure 7.1. The first column of the table shows the time based calculations of the state of charge at $20 \%$ intervals. The second column lists the state of charge generated by the coulometric algorithms. Next to this is the error in percent between the two state of charge values. There is a good agreement between the two sets of state of charge values although the coulometric state of charge was under predicted by a small amount. The maximum error occurred at $40 \%$ SOC and was $-0.6 \%$. Due to the way the coulometric calculations are made, errors will tend to accumulate. This is shown in table 7.1 where the error tended to be greater as the state of charge decreased. The average error over the whole discharge was $-0.3 \%$.

\begin{tabular}{|c|c|c|}
\hline $\begin{array}{c}\text { Time based } \\
\text { SOC (\%) }\end{array}$ & \multicolumn{2}{c|}{$\begin{array}{c}\text { Coulometric } \\
\text { SOC (\%) }\end{array}$} \\
\hline Error (\%) \\
\hline 100 & 100 & 0 \\
\hline 80 & 79.9 & -0.1 \\
\hline 60 & 59.7 & -0.3 \\
\hline 40 & 39.4 & -0.6 \\
\hline 20 & 19.5 & -0.5 \\
\hline 0 & -0.3 & -0.3 \\
\hline
\end{tabular}

Table 7.1: A comparison of time based and coulometric SOC results during a discharge at a rate of $7.2 \mathrm{~A}$.

The results from the remaining tests at the higher discharge tests are summarised in tables $7.2,7.3,7.4$ and 7.5 for the $18 \mathrm{~A}, 36 \mathrm{~A}, 54 \mathrm{~A}$ and $72 \mathrm{~A}$ discharge tests respectively. 


\begin{tabular}{|c|c|c|}
\hline Time based & Coul & ietric \\
\hline $\operatorname{soc}(\%)$ & SOC $(\%)$ & Error (\%) \\
\hline 100 & 100 & 0 \\
\hline 80 & 79.8 & -0.2 \\
\hline 60 & 59.3 & -0.7 \\
\hline 40 & 39.2 & -0.8 \\
\hline 20 & 19.5 & -0.5 \\
\hline 0 & -0.6 & -0.6 \\
\hline
\end{tabular}

Table 7.2: Coulometric results during a 18A discharge test.

\begin{tabular}{|c|c|c|}
\hline $\begin{array}{c}\text { Time based } \\
\text { SOC (\%) }\end{array}$ & \multicolumn{2}{c|}{$\begin{array}{c}\text { Soculometric } \\
\text { SO) }\end{array}$} \\
\hline 100 & 100 & 0 \\
\hline 80 & 78.0 & -2 \\
\hline 60 & 58.6 & -1.4 \\
\hline 40 & 36.9 & -3.1 \\
\hline 20 & 17.5 & -2.5 \\
\hline 0 & -4.2 & -4.2 \\
\hline
\end{tabular}

Table 7.4: Coulometric results during a 54 discharge test.

\begin{tabular}{|c|c|c|}
\hline $\begin{array}{c}\text { Time based } \\
\text { SOC (\%) }\end{array}$ & \multicolumn{2}{c|}{ Coulometric } \\
\hline 100 & 100 & Error (\%) \\
\hline 80 & 80.0 & 0 \\
\hline 60 & 59.7 & -0.3 \\
\hline 40 & 40.5 & 0.5 \\
\hline 20 & 20.1 & 0.1 \\
\hline 0 & -1.1 & 1.1 \\
\hline
\end{tabular}

Table 7.3: Coulometric results during a $36 \mathrm{~A}$ discharge test.

\begin{tabular}{|c|c|c|}
\hline $\begin{array}{c}\text { Time based } \\
\text { SOC (\%) }\end{array}$ & \multicolumn{2}{c|}{ Coulometric } \\
SOC(\%) & Error (\%) \\
\hline 100 & 100 & 0 \\
\hline 80 & 78.7 & -1.3 \\
\hline 60 & 60.5 & 0.5 \\
\hline 40 & 39.6 & -0.4 \\
\hline 20 & 18.2 & -1.8 \\
\hline 0 & -1.3 & -1.3 \\
\hline
\end{tabular}

Table 7.5: Coulometric results during a $72 \mathrm{~A}$ discharge test.

In general there is a good agreement between the results shown in tables 7.2 to 7.5 although the errors are slightly higher than in the 7.2A test. This is most likely due to the fact that adjustable load banks were used rather than a constant current sink. The higher current discharge tests, particularly the 54A test, show slightly higher errors than the other tests. At the higher discharge rates the current tends to drift more during the discharge and is hence more difficult to manually keep at the desired value, contributing to higher errors in the results.

The average error in each discharge test is summarised in table 7.6. In each of these tests the battery monitor on average under predicted the state of charge. The highest average error occurred in the $54 \mathrm{~A}$ discharge test at $-2.2 \%$. In most of the other tests the error was equal to or below $-0.5 \%$. The main other exception to this being the $72 \mathrm{~A}$ test which had an average error of $-0.7 \%$. This suggests that the approximated relation between the discharge current and battery capacity made by the Peukert equation (equation 3.1) is reasonably accurate. The errors in the 54A test are likely to have been caused by external sources. 


\begin{tabular}{|c|c|}
\hline $\begin{array}{c}\text { Discharge } \\
\text { Rate (A) }\end{array}$ & $\begin{array}{c}\text { Average } \\
\text { Error (\%) }\end{array}$ \\
\hline 7.2 & -0.3 \\
\hline 18 & -0.5 \\
\hline 36 & -0.1 \\
\hline 54 & -2.2 \\
\hline 72 & -0.7 \\
\hline
\end{tabular}

Table 7.6: The average error in each of the five discharge tests.

\subsubsection{Low Temperature Results}

The discharge tests reported in section 7.2.1 were all carried out at room temperature. In order to test the performance of the battery monitor under low battery temperature conditions, discharge tests were carried out on a battery that had been chilled to $5^{\circ} \mathrm{C}$. This was achieved using the experimental setup shown in figure 7.2.

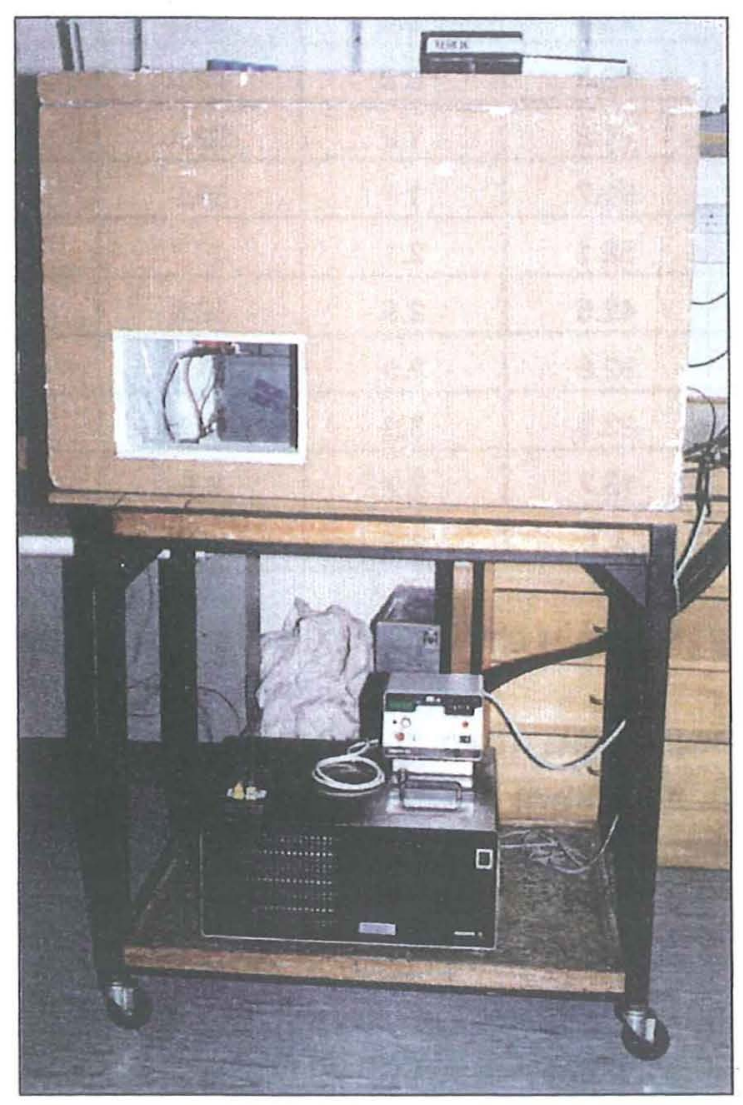

Figure 7.2: $\quad$ The insulated container and temperature controlling unit used to perform the low temperature battery monitor testing. 
The battery was placed in an insulated container, the brown box in figure 7.2. A Haake temperature controlling unit, the black box at the bottom of figure 7.2, was used to achieve the cooling. This device contains a heat pump and a heating unit. Coolant, in this case water, is pumped through the heat exchanger in the unit and out to a second heat exchanger in the insulated container. The Haake unit measures and controls the temperature of the coolant. The temperature is set on the control box on top of the Haake unit.

For these tests a temperature of $2^{\circ} \mathrm{C}$ was selected on the Haake in order to achieve a stable temperature of $5^{\circ} \mathrm{C}$ in the insulated container. Discharge tests were carried out a discharge rate of 7.2A $\left(\mathrm{C}_{5}\right)$, which is low enough to prevent significant internal battery heating from occurring that could upset the results. As before the battery was initially fully discharged at this temperature to measure the total discharge time in order to allow the state of charge to be calculated based on time measurements. The results from this test are summarised in table 7.7 taken at $10 \%$ SOC decrements.

\begin{tabular}{|c|c|c|c|c|}
\hline $\begin{array}{c}\text { Time based } \\
\text { SOC (\%) }\end{array}$ & \multicolumn{2}{c}{ Coulometric } & \multicolumn{2}{c|}{ Coulometric with T comp } \\
\hline 100 & 100 & 0 & 100 & 0 \\
\hline 90 & 90.4 & 0.4 & 89.9 & -0.1 \\
\hline 80 & 80.8 & 0.8 & 79.9 & -0.1 \\
\hline 70 & 71.2 & 1.2 & 69.8 & -0.2 \\
\hline 60 & 61.7 & 1.7 & 59.8 & -0.2 \\
\hline 50 & 52.1 & 2.1 & 49.7 & -0.3 \\
\hline 40 & 42.5 & 2.5 & 39.6 & -0.4 \\
\hline 30 & 32.9 & 2.9 & 29.6 & -0.4 \\
\hline 20 & 23.3 & 3.3 & 19.5 & -0.5 \\
\hline 10 & 13.7 & 3.7 & 9.5 & -0.5 \\
\hline 0 & 4.1 & 4.1 & -0.6 & -0.6 \\
\hline
\end{tabular}

Table 7.7: Results obtained from the low battery temperature tests.

The state of charge measurements from the battery monitor, both with and without temperature compensation, are shown in table 7.7. In both cases the error in percent has been calculated between the state of charge values and the time based state of charge. As expected, without temperature compensation there is a higher error than with temperature compensation. Without temperature compensation the state of charge was over predicted and showed $4.1 \%$ at the end of the discharge. With temperature compensation the state of charge was slightly under predicted, showing $-0.6 \%$ at the end of the discharge. The average error over the entire discharge was $2.1 \%$ with no compensation for temperature and was $-0.3 \%$ with compensation. This demonstrates that the temperature compensation improves the battery monitor results at low temperatures. 


\subsubsection{Charging Results}

The final test of the coulometric algorithms involved monitoring a charge cycle. This test was performed on a fully discharged battery which was charged using the constant current-constant voltage (I-V) charging method described in section 2.6.3. The battery was charged under similar conditions to that which are present in the Department's electric car although this currently uses constant voltage (V) charging from a rectified mains supply. The main objective of this test was to confirm that the coulometric calculations accurately estimated the state of charge during charging, and hence also allowed the validity of assumptions made about the battery charge efficiency to be found.

The battery was initially charged at a current of $8 \mathrm{~A}$ which was switched to a float charge once the battery voltage had reached $14.2 \mathrm{~V}$. The logging function of the monitor was used in this test to log charge current, voltage, battery temperature, the returned amperehours and the state of charge during discharge. Figure 7.3 shows the results obtained.

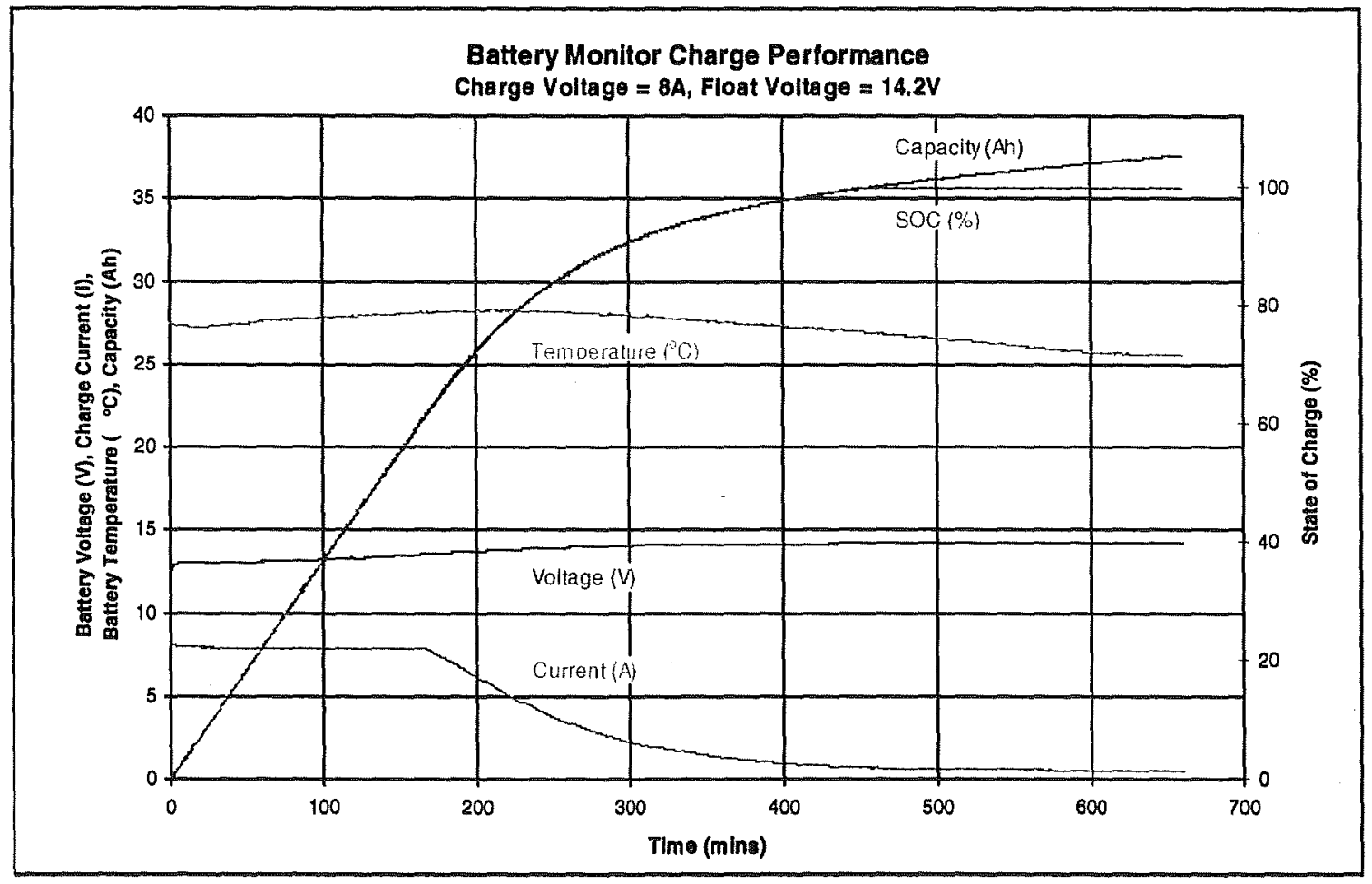

Figure 7.3: The results logged by the battery monitor during a charge cycle.

The charge voltage and current characteristics agree very well with the typical characteristics shown in figure 2.5 for this type of charging. The temperature reached a peak shortly after the charger switched to constant voltage charging and then began to fall off. This corresponds to the falling off of the charge current and hence less heat being dissipated in the battery itself. The battery temperature at the end of the charge is lower than at the start since the battery had just finished discharging at the start of the charge cycle and also, since this test was carried out overnight, the falling ambient temperature would also have had some influence. 
The state of charge and hence the battery capacity both rise linearly while the charge current is constant. During the constant voltage section of the charge both the state of charge and the capacity increase at a gradually decreasing rate. The state of charge stops increasing once $100 \%$ is reached while the capacity continues to increase slightly until the end of the discharge is reached. This is due to an internal limit in the software to prevent the state of charge exceeding $100 \%$. Full charge is actually reached when the charge voltage reaches the set float voltage and the charger is only supplying a small, near constant residual current to the battery. This occurs after just over 8 hours (about 500 minutes). The state of charge calculation reaches $100 \%$ slightly before this, however since the charge current is very low (around $0.5 \mathrm{~A}$ ) at this point only a very small error occurs. At the point where the battery is fully charged 36.3Ah have been returned to the battery. The state of charge algorithms assume, during charging, that the battery is fully recharged after $36 \mathrm{Ah}$ have been returned. This small discrepancy amounts to an error of less than $1 \%$, which is acceptable.

\subsubsection{Open Circuit Voltage Results}

Once the coulometric algorithms had been fully tested the open circuit voltage algorithms described in section 6.4 .4 were put through a similar set of tests. As the open circuit algorithms cannot monitor the battery while it is being discharged, these tests were performed by discharging the battery to a predetermined state of charge. The load was then disconnected so that the open circuit voltage and hence the state of charge could be predicted. These results were then compared to the results obtained from the coulometric state of charge measurements. This procedure was repeated at regular intervals until the battery was fully discharged. Since the battery was rested during the five minute intervals required to collect the open circuit voltage measurements the recuperation compensated state of charge measurements were also recorded for comparison. Like the previous coulometric tests, these tests were carried out at several different discharge rates. Discharge rates of $7.2 \mathrm{~A}\left(\mathrm{C}_{5}\right), 18 \mathrm{~A}\left(\mathrm{C}_{2}\right)$ and $54 \mathrm{~A}(1.5 \mathrm{C})$ were used.

Table 7.8 shows the results obtained during the 7.2A discharge. As in the previous tests the SOC calculation based on time was used as a reference. The coulometric SOC results, both with and without compensation for battery recuperation, are shown for comparison along with the predicted values for both the open circuit voltage (OCV) and state of charge. The error between this value and the theoretical (time based) state of charge is also shown, as well as the predicted state of health ( $\mathrm{SOH}$ ) of the battery in percent. 


\begin{tabular}{|c|c|c|c|c|c|c|}
\hline \multirow{2}{*}{$\begin{array}{c}\text { Time based } \\
\text { SOC }(\%)\end{array}$} & $\begin{array}{c}\text { Coulometric SOG (\%) } \\
\text { Standard }\end{array}$ & Recuperation & OCV (V) & SOC (\%) & $(\%)$ & $(\%)$ \\
\hline 80 & 79.5 & 79.5 & 12.97 & 83.3 & 3.3 & 120 \\
\hline 60 & 59.3 & 59.6 & 12.70 & 60.5 & 0.5 & 117 \\
\hline 40 & 39.2 & 39.9 & 12.50 & 44.6 & 4.6 & 129 \\
\hline 20 & 18.6 & 19.9 & 12.24 & 22.4 & 2.4 & 129 \\
\hline 0 & 0.0 & 0.0 & 12.03 & 4.1 & 4.1 & - \\
\hline
\end{tabular}

Table 7.8: $\quad$ Open circuit voltage results obtained during a 7.2A discharge.

From table 7.8 it can be seen that the recuperation compensated SOC results are closer to the time based SOC results suggesting that the battery is in fact regaining some of its capacity in the rest periods required to make the open circuit voltage measurements. The SOC results from the open circuit voltage measurements vary in accuracy when compared to the time based SOC. In each case the SOC is over predicted with the maximum error occurring at $40 \%$ SOC with a $4.6 \%$ over prediction. The lowest error of $0.5 \%$ occurred at $60 \%$ SOC. The average error for these results is $3 \%$.

The state of health results in table 7.8 show that the battery is delivering a higher capacity than that predicted by manufacturer's data, which is consistent with a new battery. The state of health predictions vary between $117 \%$ and $129 \%$ at the four different measurement points with an average of $124 \%$. The SOH could not be calculated at $0 \%$ SOC since the coulometric SOC is $0 \%$ which would cause a divide by zero error in the calculations. There is a noticeable increase in the $\mathrm{SOH}$ results as the battery is discharged further.

\begin{tabular}{|c|c|c|c|c|c|c|}
\hline Time based & \multicolumn{2}{|c|}{ Coulometric SOC $(\%)$} & Predicted & Predicted & Error & $\mathrm{SOH}$ \\
\hline $\operatorname{soc}(\%)$ & Standard & Recuperation & $\operatorname{OcV}(V)$ & SOC $(\%)$ & $(\%)$ & $(\%)$ \\
\hline 80 & 79.8 & 79.9 & 12.97 & 83.8 & 3.8 & 121 \\
\hline 60 & 59.6 & 60.3 & 12.74 & 64.1 & 4.1 & 122 \\
\hline 40 & 39.8 & 39.9 & 12.51 & 44.8 & 4.8 & 129 \\
\hline 20 & 19.7 & 20.5 & 12.24 & 22.1 & 2.1 & 124 \\
\hline 0 & 5.6 & 6.6 & 12.03 & 4.4 & 4.4 & 77 \\
\hline
\end{tabular}

Table 7.9: Open circuit voltage results obtained during a 18A discharge.

Table 7.9 shows open circuit voltage results obtained during an 18A discharge. These results are similar to the results obtained for the $7.2 \mathrm{~A}$ discharge although the errors are slightly higher. Again the state of charge is slightly over predicted with an average error in the open circuit voltage SOC of $3.8 \%$. There is also a small anomaly in the coulometric results with both the uncompensated and recuperation compensated measurements still showing $5.6 \%$ and $6.6 \%$ at $0 \%$ SOC. Since the state of charge is not zero at this point a SOH calculation could be made, although at $77 \%$ it is considerably lower than the other SOH results. This is due to the fact that at this low state of charge 
the errors are quite significant compared to the magnitude of the numbers. As a result the state of health at this state of charge has a significant error and should not be used. The average state of health excluding this last result is $124 \%$.

\begin{tabular}{|c|c|c|c|c|c|c|}
\hline \multirow{2}{*}{$\begin{array}{l}\text { Time based } \\
\text { SOC }(\%)\end{array}$} & \multicolumn{2}{|c|}{ Coulometric SOC (\%) } & \multirow{2}{*}{$\begin{array}{l}\text { Predicted } \\
\text { OCV (V) }\end{array}$} & \multirow{2}{*}{$\begin{array}{l}\text { Predicted } \\
\text { SOC }(\%)\end{array}$} & \multirow{2}{*}{$\begin{array}{l}\text { Error } \\
(\%)\end{array}$} & \multirow{2}{*}{$\begin{array}{l}\mathrm{SOH} \\
(\%)\end{array}$} \\
\hline & Standard & Recuperation & & & & \\
\hline 80 & 79.5 & 79.7 & 12.95 & 81.6 & 1.6 & 118 \\
\hline 60 & 59.0 & 60.8 & 12.75 & 65.1 & 5.0 & 123 \\
\hline 40 & 36.4 & 40.4 & 12.50 & 43.6 & 3.6 & 124 \\
\hline 20 & 14.4 & 20.4 & 12.25 & 22.6 & 2.6 & 127 \\
\hline 0 & 0 & 2.0 & 12.02 & 3.2 & 3.2 & - \\
\hline
\end{tabular}

Table 7.10: Open circuit voltage results obtained during a 54A discharge.

The final set of open circuit voltage results are shown in table 7.10. These results show a higher discrepancy between the uncompensated and compensated coulometric results. This is due to the fact that the amount of capacity regained at higher discharge currents is more significant than at lower discharge currents. Also since the discharge is at a higher rate the total discharge time is shorter and hence the fixed length reset periods make up a higher proportion of the total time of the test.

The open circuit voltage SOC results are similar to the previous results. The average error in this case was $3.2 \%$ although there was a large error of $5 \%$ at $60 \%$ SOC. The state of health results were again similar with an average $\mathrm{SOH}$ of $123 \%$.

There may be other factors contributing to the observed errors in the SOC results from open circuit voltage predictions. One possible source of error may have been caused by variations in the charge cycle. Some charge cycles were left overnight before the charger was turned off whereas other charge cycles were terminated straight after the charge was thought to have finished. Errors contributed from this possible source are likely to be small and only in the order of one or two percent.

\subsubsection{State of Health Testing}

To conclusively bench test the battery state of health monitoring, a new battery would have to be cycled though a large number of discharge cycles until it failed. This would give a good picture of what was happening over the lifespan of the battery, but would have taken a long time to perform and would have destroyed a good battery. For these reasons bench testing of the state of health monitoring was not performed. The state of health results obtained in the previous section were however, at around $124 \%$, reasonably consistent with what would be expected from a new battery. An older battery that has - reached the end of its useable life would be expected to give a state of health of below $80 \%$. Testing of the state of health monitoring will be able to be done in the future in the electric car itself, over the lifespan of the car's batteries. 


\subsection{Electric Car Installation}

Once the bench testing was complete, the battery monitor was installed in the Department's electric car. Only one BMM was installed for initial testing, allowing only the first six batteries in the bank to be monitored. Figure 7.4 shows the BMM installed in the under bonnet battery compartment of the car. The connections from the batteries to the BMM were made via fused leads. The temperature sensor was attached to the side of one of the batteries in the compartment.

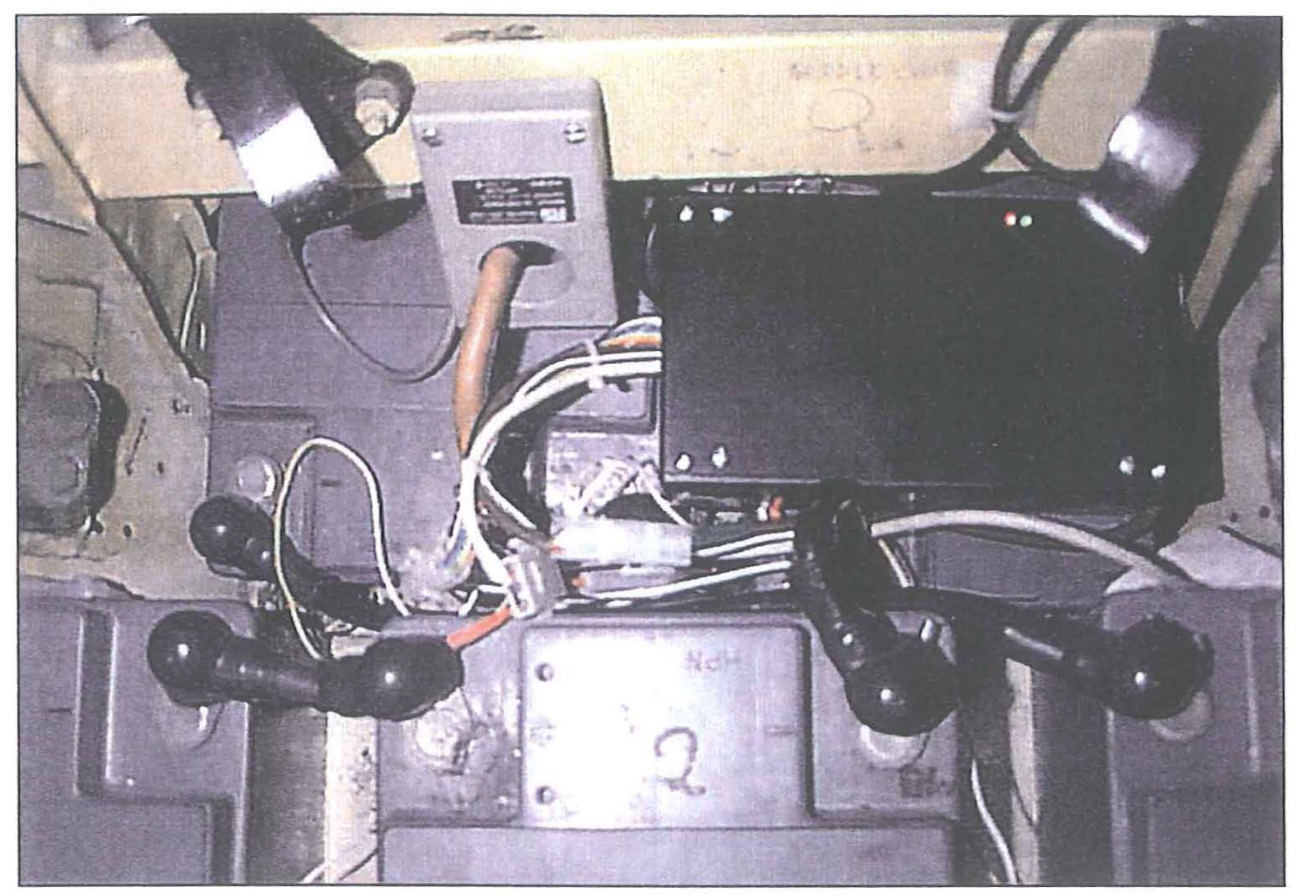

Figure 7.4: One of the BMMs shown mounted in the electric car. The current sensor is also visible to the left of the BMM box.

Two cables run from the BMM to the interior of the car. One is the serial communication cable connecting the BMM to the battery monitor and the other connects the BMM to the current sensor via the board on the rear of the car's current meter.

The main battery monitor box was mounted under the car's dashboard. The main and backup supplies were derived from an existing separate $12 \mathrm{~V} 15 \mathrm{Ah}$ battery used as a backup to the car's auxiliary supply. The main supply to the monitor is switched through a set of spare contacts on an existing relay, controlled from the car's 'ignition' switch.

The two monitor displays were mounted on the car's dashboard. The LCD display board was mounted in a central position on the car's dashboard (figure 7.5) and the state of charge display board was mounted in the redesigned instrument panel (figure 7.6). 


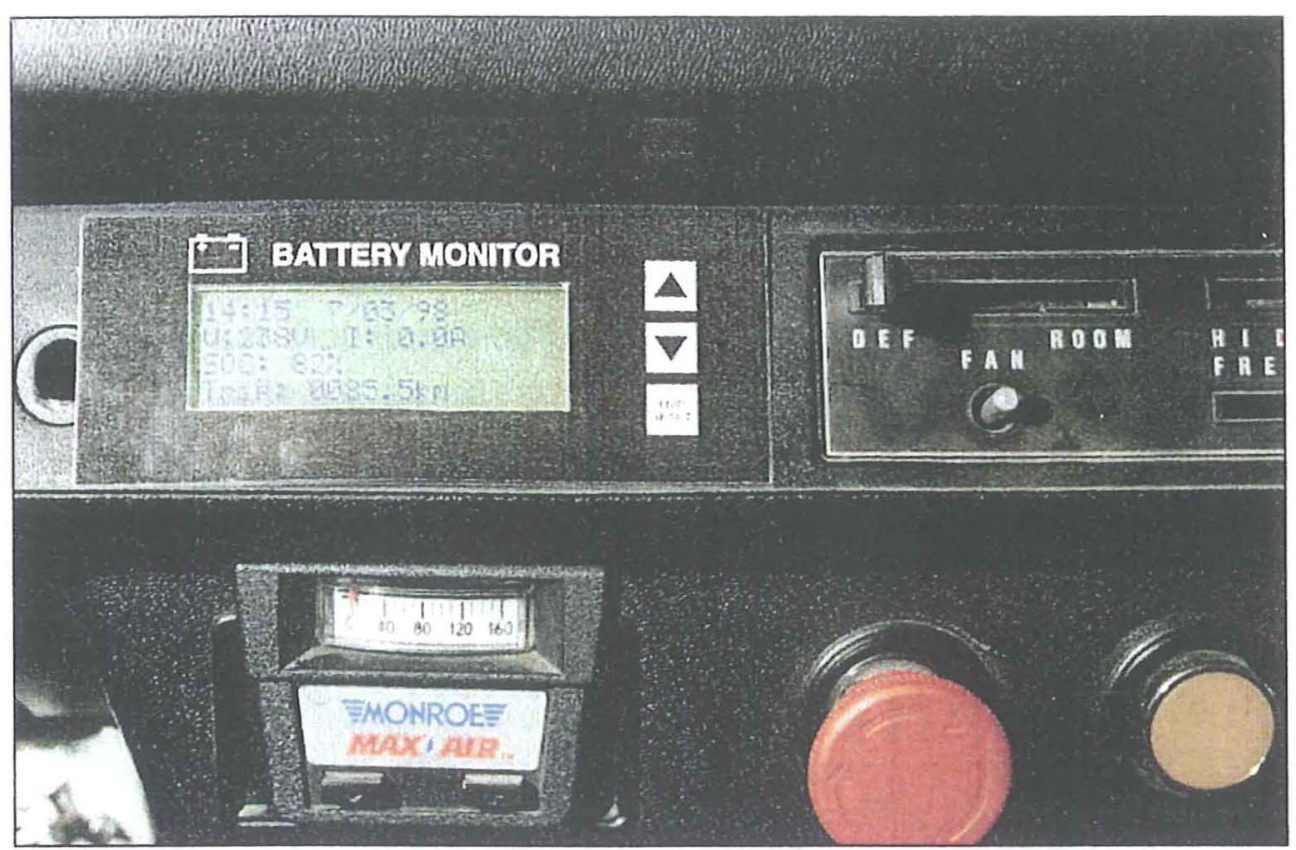

Figure 7.5: The LCD display mounted in the car.

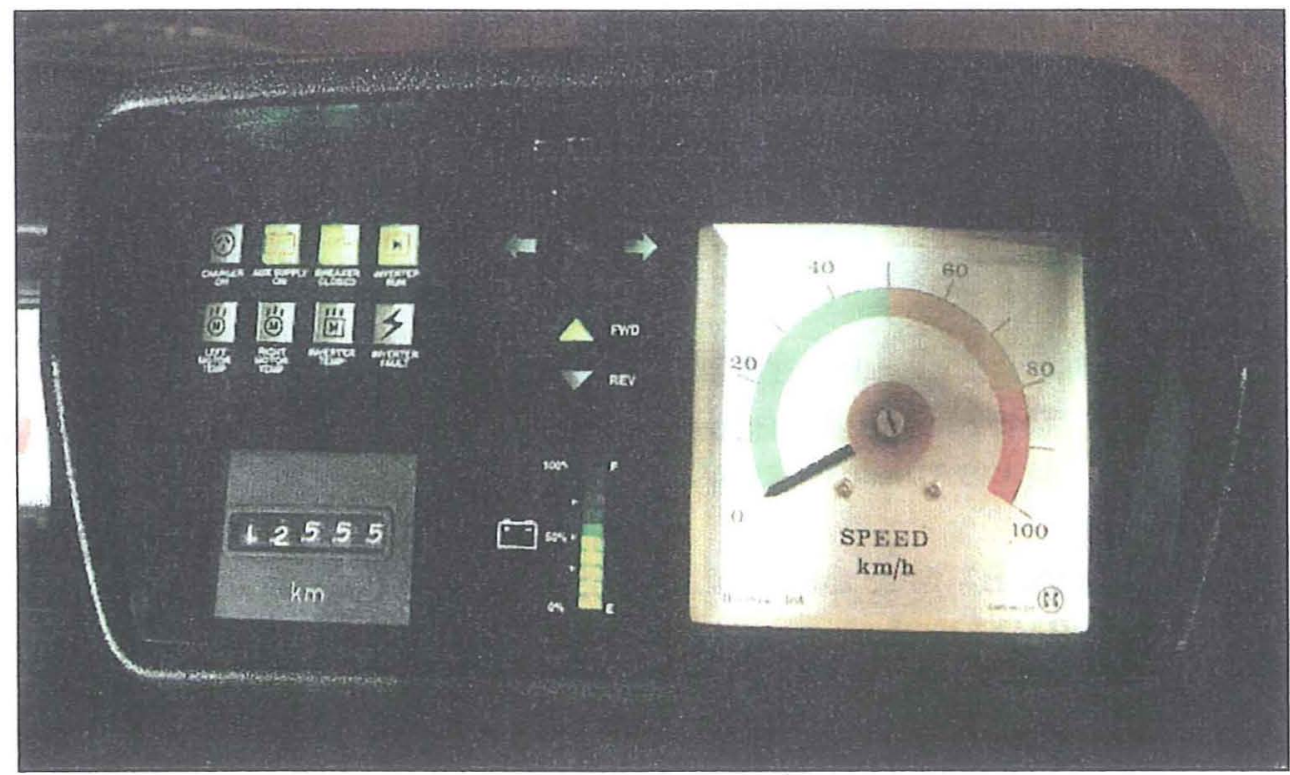

Figure 7.6: The updated instrument panel.

\subsection{Road Tests}

The main objective of the road tests were to test the overall functionality of the monitor in the electric car. These tests were designed to check that the monitor state of charge predictions were accurate and did not significantly over or under predict with the electrical loads present in the car. They would also allow the distance prediction algorithms to be tested and their accuracy determined. 
In order to perform repeatable road tests, testing was to be performed with the car on a dynamometer. This would provide a fixed load to the motors that would be similar to what would be expected during typical driving conditions on the flat. Several different tests would have been performed, simulating different types of driving conditions and driving cycles.

The first set of tests would have involved running the car at constant speeds on fully charged batteries until the batteries were flat. Speeds of $30 \mathrm{~km} / \mathrm{h}, 50 \mathrm{~km} / \mathrm{h}$ and $70 \mathrm{~km} / \mathrm{h}$ would have been used. Over the duration of each test the battery monitor would have been used to log data for later analysis. As well as demonstrating the accuracy of the state of charge algorithms and the distance prediction algorithms, these tests would have allowed valuable data on the performance and range of the car itself to be gained.

The second set of tests were designed to simulate typical urban driving conditions with a number of start/stop cycles and short rest periods. This would have allowed the performance of both the battery monitor and the car to be tested under driving conditions that the car would most likely be subjected to in everyday usage. To perform these tests in a repeatable manner a computer would have been used to provide a programmed set of control signals to the car's drive controller.

Unfortunately a catastrophic fault within the inverter occurred before these tests could be performed. The fault destroyed two of the switching transistors in the inverter. Only one spare was available and since these parts are now obsolete, additional replacements could not be obtained. This has meant that the inverter cannot be repaired and will be replaced in the future with one designed using more modern devices. As a result the road tests could not be completed.

When the inverter fault occurred it caused a voltage spike to travel though the battery bank. This spike allowed the BMM input protection circuitry, described in section 5.3.3, to be tested during an actual fault condition. The protection circuitry blew the fuses on all BMM voltage channels. It also caused four of the six zener diodes on the channel inputs to short circuit. The protection circuitry however worked and the BMM input circuitry survived the spike. On closer inspection of the BMM wiring it was discovered that the shorting of one zener diode may have caused the protection circuitry on the other channels to operate through a possible domino effect. To overcome this the battery wiring may need to be modified so that when a fuse does blow it disconnects that channel from the other channels as well as the battery. 


\section{Discussion}

\subsection{Battery Monitor Evaluation}

Bench testing of the battery monitor showed that it performed well under most conditions tested. Tests of the coulometric algorithms gave results that agreed well with the state of charge results based on time measurements. In most cases the errors in the results over a full discharge were less than $1 \%$, although the results obtained at the higher discharge currents did have higher errors due to the higher inaccuracies in the test methods. The errors in the results were mostly on the conservative side, giving a small under prediction of the state of charge in most cases. These results prove that the Peukert equation constants used in the coulometric calculations give a relationship between discharge current and battery capacity that accurately predicts the total battery capacity.

Further testing of the coulometric algorithms at low temperature and during a charge cycle gave results that were again very good. The error during a low temperature discharge test was improved by several percent when temperature compensation was used. Testing during a charge cycle showed that although there was a small over prediction of the state of charge, this was very small at less than $1 \%$.

The results obtained from the open circuit voltage prediction algorithms were not quite as accurate and consistent as those obtained from the coulometric method. Overall the open circuit voltage tended to over predict the state of charge with an average error of around $3 \%$ which is still within the desired design accuracy of $5 \%$. Since this method first makes two voltage measurements, calculates a prediction of the fully recovered open circuit voltage and finally uses this value to calculate the state of charge, there is a greater opportunity for errors to affect the results. The relationship between the open circuit voltage and the state of charge is claimed to be not significantly influenced by the temperature and past history of the battery [8]. This however may not be the case with the open circuit voltage recovery. Further investigation of the recovery characteristics may be required to allow better predictions to be made. In particular the effects of temperature and the battery state of charge on the recovery characteristic may need to be looked at.

The state of health results obtained during the open circuit voltage tests appeared to be consistent with a new battery that was performing better than the manufacturer's data would predict. The state of health monitoring tended to give better results when the state of charge was above $50 \%$. This is thought to be due to the fact that the state of charge errors become more significant at lower states of charge. Since the state of health 
calculations are based on the state of charge calculations, any errors in the state of charge calculations will propagate through to the state of health results. Due to the time required to perform conclusive state of health testing over the lifespan of a battery, further state of health testing was not performed.

\subsection{Future Testing}

Due to a failure within the inverter of the Department's electric car, testing of the monitor in the car could not be carried out. These tests would have allowed the distance prediction algorithms to be tested. It is hoped that these tests can be performed in the future, when the car is fully functional again.

The state of health monitoring was also not fully tested due to the time required to perform testing over the life time of a battery. Once the electric car is operating, information on the car's batteries' state of health can be gathered from the monitor and used to determine whether the state of health algorithms give reliable results over the lifespan of the car's batteries. As the batteries age it will also be possible to determine if the coulometric algorithms are suitably adapted.

\subsection{Future Improvements}

The battery monitor at the current time requires an external computer to operate as most of the algorithms have been implemented in Visual Basic. This has been done for development and testing purposes. The final version of the software, running entirely within the microcontroller, has not been written. Once future testing of the monitor has been completed and the correct operation of the monitor algorithms confirmed, the Visual Basic code should be transferred to the microcontroller. This would allow the battery monitor to operate as a self-contained device. An external computer would then only be required to update the battery monitor parameters and to log data from the monitor.

To do this the battery monitor algorithms may need to be made more efficient to operate in a microcontroller environment. Also, due to the high math content of the algorithms, it would be recommended to write the code using a $\mathrm{C}$ compiler rather than coding it in assembly language.

The final version of the software would need to derive its timing from an external source. For this purpose, provision has been made for the external interrupt from the RTC on the main system board to feedback to the external interrupt input of the microcontroller. This interrupt can be set to occur at regular intervals and control the program timing. The RS232 communications subroutines would also need to be rewritten so that they are driven by interrupts rather than being polled in the software. 


\subsection{Use in other Applications}

While the battery monitor has been designed to monitor batteries in cyclic use it could, with suitable modifications, be used in other applications. One such application where battery monitoring is becoming increasingly desirable is in standby and UPS battery systems. The modular nature of the battery monitor described here allows it to be easily adapted to such applications. The BMMs may need to be reconfigured to take measurements from a different number of batteries or redesigned if different battery parameters need to be measured. Also the monitoring software would need to be rewritten to meet the different monitoring requirements. 


\section{Conclusion}

This project has realised a battery monitoring system suitable for use in an electric vehicle. The monitoring system is able to calculate the state of charge of the vehicle's batteries to give what would be the equivalent of a fuel gauge in a conventionally powered vehicle. In addition to this information it also provides a prediction of the remaining distance that the vehicle may travel before it requires recharging. It is also capable of measuring the state of health of multiple batteries within an electric vehicle's battery bank and provides data logging functions.

After initially examining the characteristics of lead acid batteries and making a comparison of various battery monitoring techniques it was discovered that no one method could meet all the requirements for an electric vehicle. It was therefore decided to use a combination of two methods, the coulometric and open circuit voltage measurement methods. Combining these two methods allowed state of charge measurements to be made while the vehicle was running using the coulometric method. These measurements were then updated using the open circuit voltage measurement method, once the vehicle was powered down. This had the advantage that the measuring method could be made adaptive to account for such variables as battery ageing and could also provide state of health measurements.

Hardware was developed to perform monitoring based on these methods. The battery monitor hardware was designed in a modular way with a central processing board based around a PIC microcontroller. The battery measurements were made using battery measurement modules located in the battery compartments themselves. Two displays were used to convey information to the user. These consisted of an LED bargraph display to display the battery state of charge and an alphanumeric LCD panel to display more detailed information.

For development and testing purposes the majority of the software was implemented in Visual Basic on an external computer connected to the battery monitor. This was done to speed up the development of the battery monitor software. Code for the microcontroller was written to provide the interface between the external computer and the various hardware devices within the battery monitor. In the future, once full testing of the monitor can be performed and the software has been fine tuned, the software can be rewritten for the microcontroller so that no external computer is required.

Testing of the battery monitor on the bench has shown that the state of charge algorithms give results of a good accuracy over a range of discharge currents, at low temperatures 
and during battery charging. The open circuit voltage predictions gave results that were reasonably accurate although they could be improved to reduce their errors further.

Testing of the distance remaining predictions could not be made due to a failure of the inverter in the Department's electric car. The state of health prediction could also not be tested due to the time required to collect measurements over the lifespan of a battery. It is hoped that these tests will be performed sometime in the future.

Overall this project has shown that designing a battery monitoring system is not a trivial task. Future work on this project will allow full testing of the battery monitor and allow the software to be developed so that a self contained battery monitoring system can be realised. 


\section{References}

1. Chan, C.C. and K.T. Chau, An Overview of Power Electronics in Electric Vehicles, IEEE Transactions on Industrial Power Electronics, 1997. 44(1): p. 3-13.

2. Riezenman, M.J., Electric Vehicles, IEEE Spectrum. 1992. p. 18-21.

3. Riezenman, M.J., EV watch: EVl hits the streets, in IEEE Spectrum. 1996. p. 20.

4. Williams, M., A Controlled Current Inverter for an Electric Vehicle, Department of Electrical Engineering. 1993, University of Canterbury: Christchurch.

5. Riezenman, M., J., The great battery barrier, IEEE Spectrum. 1992. p. 97-101.

6. Sonnenschein, Dryfit Technical Manual.

7. Patil, P.B. Fuel Gauges for Electric Vehicles, 17th IECEC. 1982. New York: IEEE.

8. Aylor, J.H., A. Thieme, and B.W. Johnson, A Battery State-of-Charge indicator for Electric Wheelchairs, IEEE Transactions on Industrial Electronics, 1992. 39(5): p. 398-409.

9. Linden, D., Handbook of Batteries, 2nd ed. 1995, USA: McGraw-Hill.

10. Japan Storage Battery Co. Ltd., Portalec PE Series, . 1988: Tokyo, Japan.

11. Churchill, T.L., J.S. Edmonds, and C.T. Feyk. Comprehensive Noninvasive Battery Monitoring of Lead-Acid Storage Cells in Unattended Locations, Telecommunications Energy Conference, INTELEC '94. 1994. Vancouver, Canada: IEEE.

12. Yamanaka, M., et al. A Life Indicator of Stationary Type Sealed Lead-Acid Battery, INTELEC'91. 1991: IEEE.

13. Maxim Integrated Products, MAX186 Low Power, 8-Channel, Serial 12-bit ADCs. 1995, Sunnyvale, CA: Maxim Integrated Products.

14. Micro Chip Technology Inc., Microchip Data Book. 1996, USA: Microchip Technology Inc. 


\section{Appendix A. Battery Data Logger}

To facilitate measurement of the battery characteristics described in chapter 3 a data logging system was developed. The logging system needed to be able to perform a controlled discharge on a battery while allowing data to be logged both during the discharge and after the discharge. This was so that both the discharge characteristics and open circuit voltage characteristics could be measured. The system was also used to control the discharge cycles during bench testing of the battery monitor described in chapter 7 .

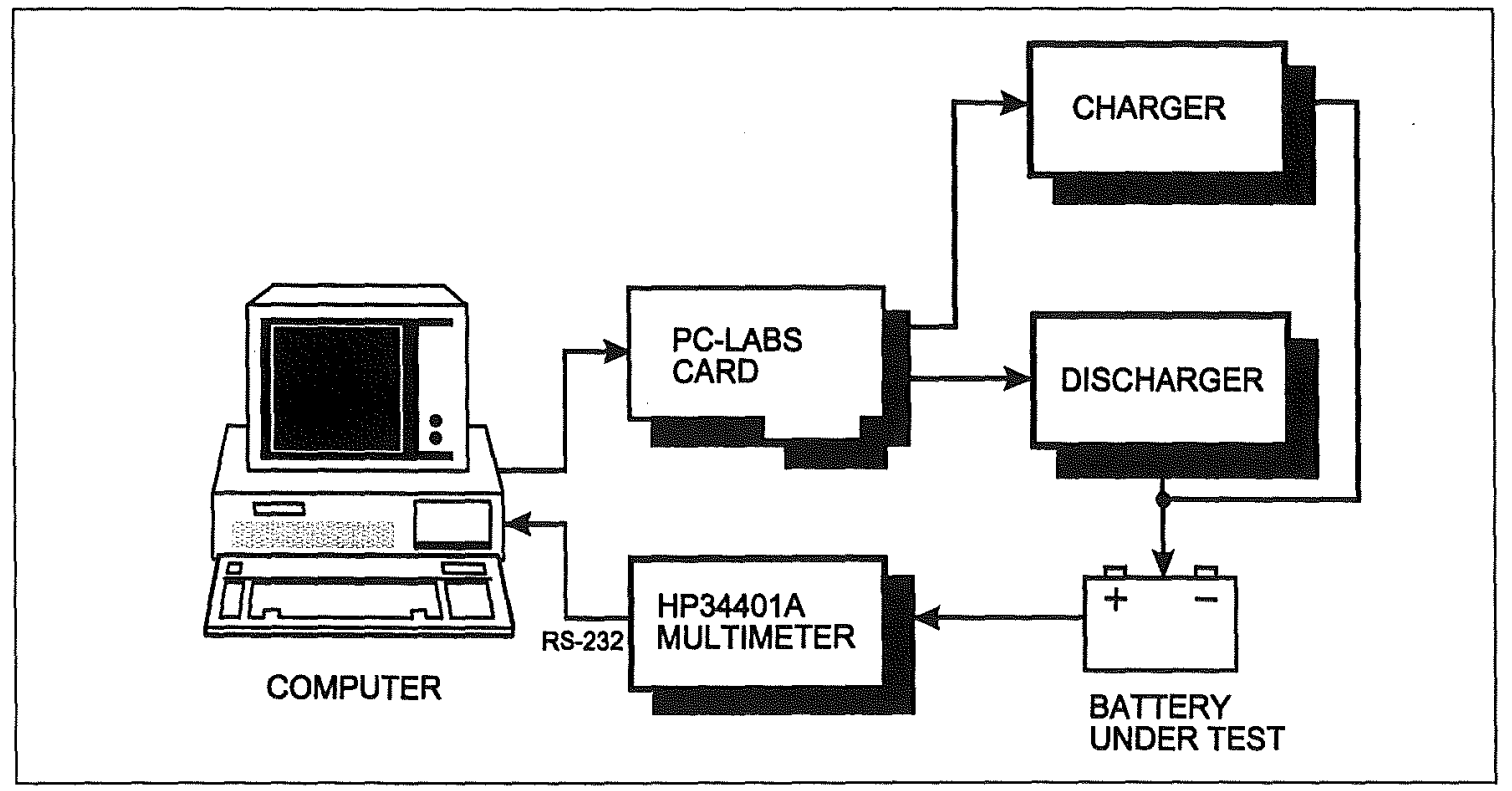

Figure A1: Block diagram of the battery data logging system.

Figure A1 shows a block diagram of the battery logging system. A computer was used to control the discharge and charge cycles and also to collect and save the measured data. A HP34401A digital multimeter was used to measure the voltage across the battery. This type of multimeter has an RS-232 and an HPIB interface. The RS-232 interface was used to interface the multimeter to the computer and hence allow collection of battery voltage measurements.

A constant current discharger was used for most of the discharge tests although, for the higher current tests, resistive load banks were used. The discharger circuit is controlled by a logic signal from a PC-Labs data-acquisition card installed in the computer. A second logic signal from this card is used to switch on and off the charger which consists of a Topward TPS-2000 power supply setup up to allow constant I-V charging.

Figure A2 shows the main components of the logging system. 


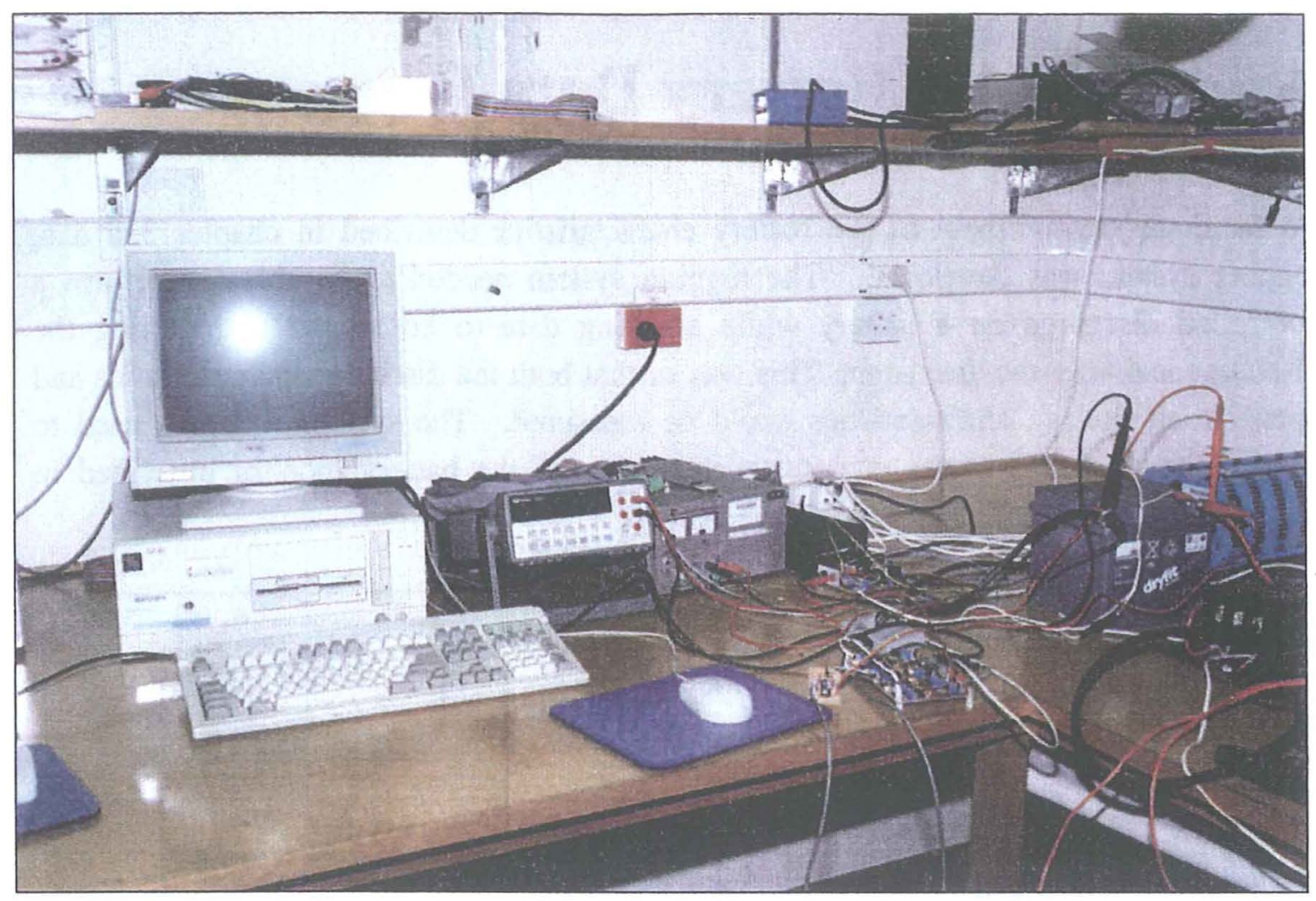

Figure A2: The logging system in operation.

\section{A1. Logging Software}

Custom software written in Visual Basic for DOS was used to perform both the control and logging functions of the logger. The software allows both the start and end times of the discharge and the period over which data logging should be performed to be specified. The discharge can also be terminated when a specified voltage is reached to allow discharges down to the battery cut-off voltage. The time after the start of the logging cycle that the charger should be switched on can also be specified to allow completely automated testing to be performed. A description of the battery test, the logging interval and the file name of the data file can also be specified for logging purposes. The logged data is saved as a comma delimited CSV file that can be directly loaded into a spreadsheet for analysis.

Figure A3 shows a screen capture of the main screen of the logger. The battery voltage and elapsed time since the logger was started are shown at the top of the screen. Below this the state of the discharger and logger are shown. A summary of the entered parameters is shown below this which includes the discharger and logger start and stop times, the minimum battery voltage that can be allowed and the filename of the data file. The bottom of the screen contains the logger control buttons. The left-most button is used to start and stop the logger. The middle button brings up the configuration dialog box that allows the test parameters to be entered and the third button exits the program. 


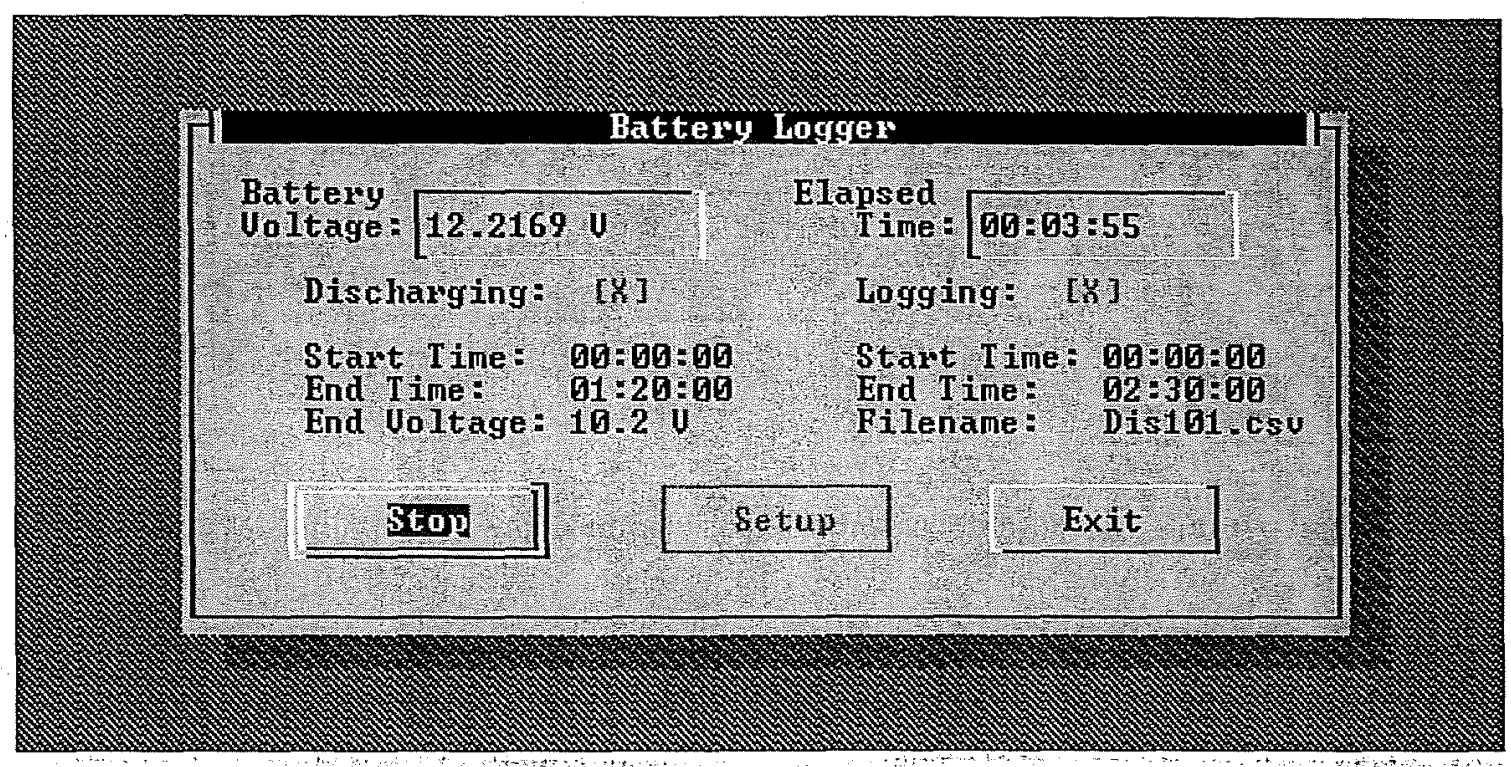

Figure A3: The main screen of the logger software.

The software polls the multimeter once very second and displays the returned measurement. Also each second the status of the Go/Stop button is checked as well as whether any of the start and end times have been reached. If they have the appropriate subroutine is called. The log interval is also checked to see if it has been reached and if it has the elapsed time in hours and the battery voltage is recorded in the data file.

\section{A2. Constant Current Discharger}

The discharger was initially designed to discharge at a constant current of 3.6A. This was later upgraded to $7.2 \mathrm{~A}$ to allow higher current discharges which allowed the discharge cycle to be done over a shorter period. The current was required to be kept constant over the operating voltage range of the battery, around 8 to $13 \mathrm{~V}$. This could not be achieved with a simple resistive load as the current would change as the terminal voltage of the battery changed.

An LM317 voltage regulator configured as a constant current sink forms the basis of the discharger circuit. Figure A4 shows how an LM317 is configured as a constant current sink.

$$
I_{S}=\frac{1.25}{R_{S}}
$$

The current is set by Rs which can be calculated using ohms law as shown in equation A1. The regulator produces a reference voltage of $1.25 \mathrm{~V}$ between the adjust and output pins which in this configuration is across resistor Rs. 


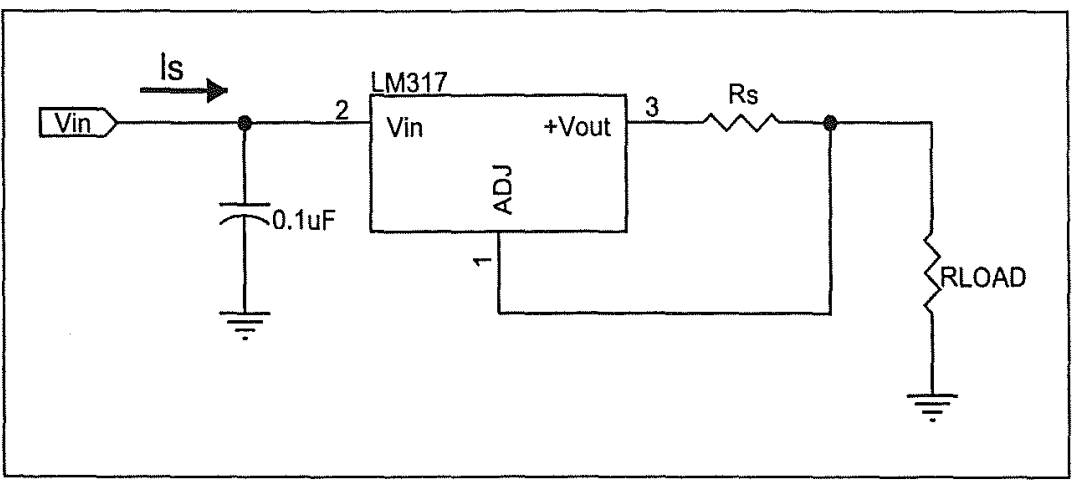

Figure A4: A constant current sink based around an LM317 voltage regulator.

Most of the power from the source is dissipated in the load resistor which must therefore have an appropriate power rating. The value of this resistor must be chosen so that at the minimum input voltage the voltage is not too high across it to prevent the designed current to flow. The voltage across this resistor is the voltage at the input to the circuit minus the voltage drop across the regulator and the drop across the current setting resistor Rs.

While this circuit provides a stable current sink it can only sink up to $1.5 \mathrm{~A}$ due to the maximum current rating of the regulator. There are two ways of getting around this limitation. One is to use a regulator with a higher current rating. The other is to use a pass transistor across the regulator to pass the current. The later option was chosen for this application. A MJ2955 PNP transistor with a current handling ability of $15 \mathrm{~A}$ was used. The final circuit of the discharger is shown in figure A5.

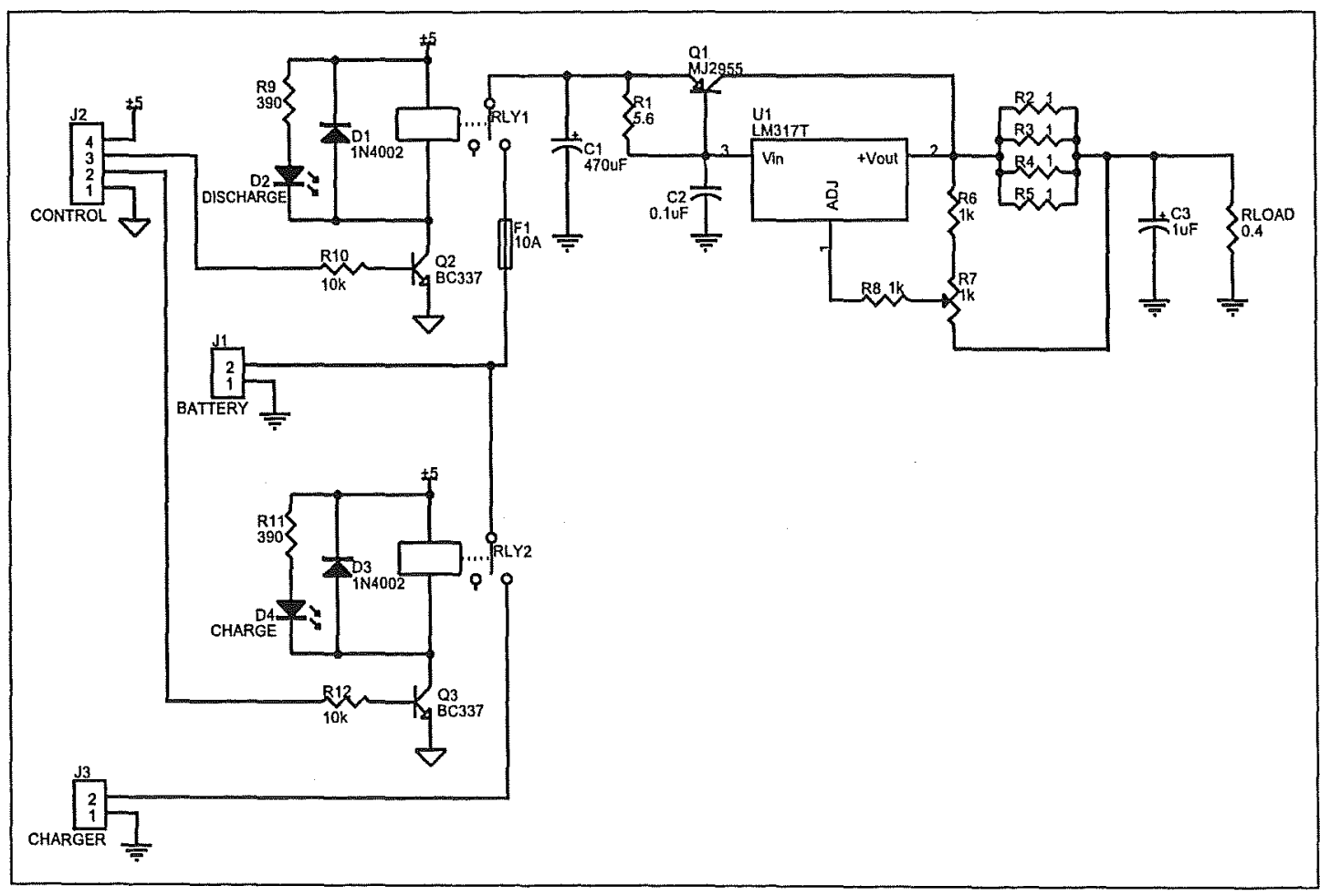

Figure A5: The final circuit of the discharger and switching relays. 
The pass transistor (Q1) is connected with its base and emitter across the input and output terminals of the regulator. In this configuration as the regulator adjusts the voltage across it to control the current, the voltage across the pass transistor also changes. The current sense resistor initially consisted of two $1 \Omega 5 \mathrm{~W}$ resistors in parallel which was later upgraded to four (R2 - R5) to configure the discharger for higher currents. In the final circuit the feedback to the adjust terminal was modified to include an adjustable voltage divider consisting of R6 and R7. This allows the reference across the current sense resistor to be effectively adjusted between $1.25 \mathrm{~V}$ and $2.5 \mathrm{~V}$ and hence allows the current flowing thought the discharger to be adjusted. Capacitors C1, C2 and C3 are used to improve the stability of the circuit.

The remaining parts of the circuit are used to control the switching of both the discharger and charger. This is achieved using two relays, RLY1 and RLY2. Relay RLY1 is used to switch power from the battery through to the discharger and RLY2 connects the battery to the charger. Both relays are switched by $5 \mathrm{~V}$ logic signals from the PC-Labs card in the PC via transistors Q2 and Q3. Both relays have reversed diodes (D1 and D3) across them to protect the transistor against back-EMFs from the relay coils. Also connected across the relays are LEDs with their associated current limiting resistors to show the status of the discharger and charger. Power for this part of the circuit comes from a separate $5 \mathrm{~V}$ power supply.

The discharger was assembled on veroboard and is shown in figure A6. The pass transistor is mounted on the separate heatsink next to the board.

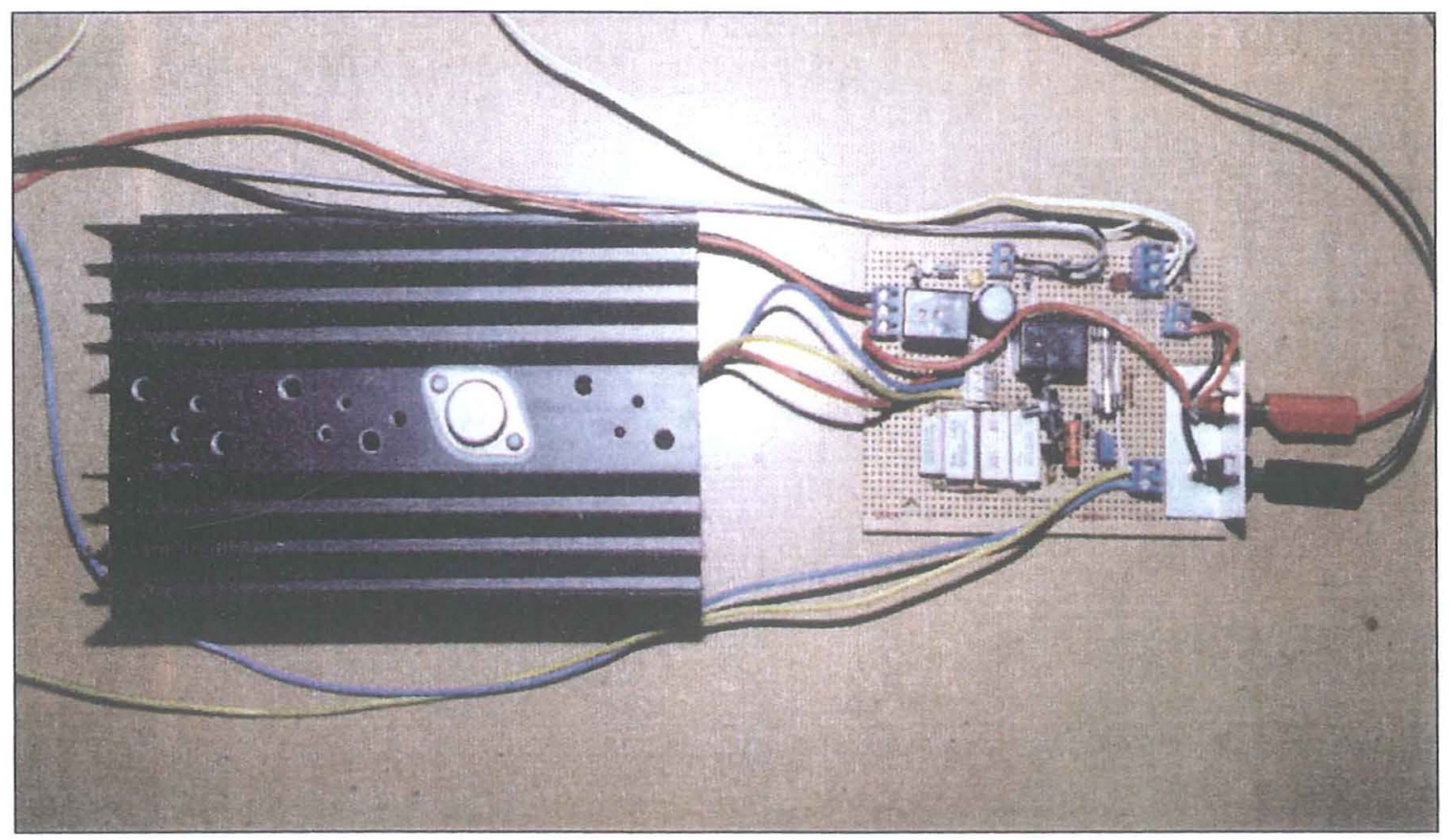

Figure A6: The assembled discharger. 


\section{Appendix B. Circuit Schematics}

\section{B1. Battery Measurement Module 1}

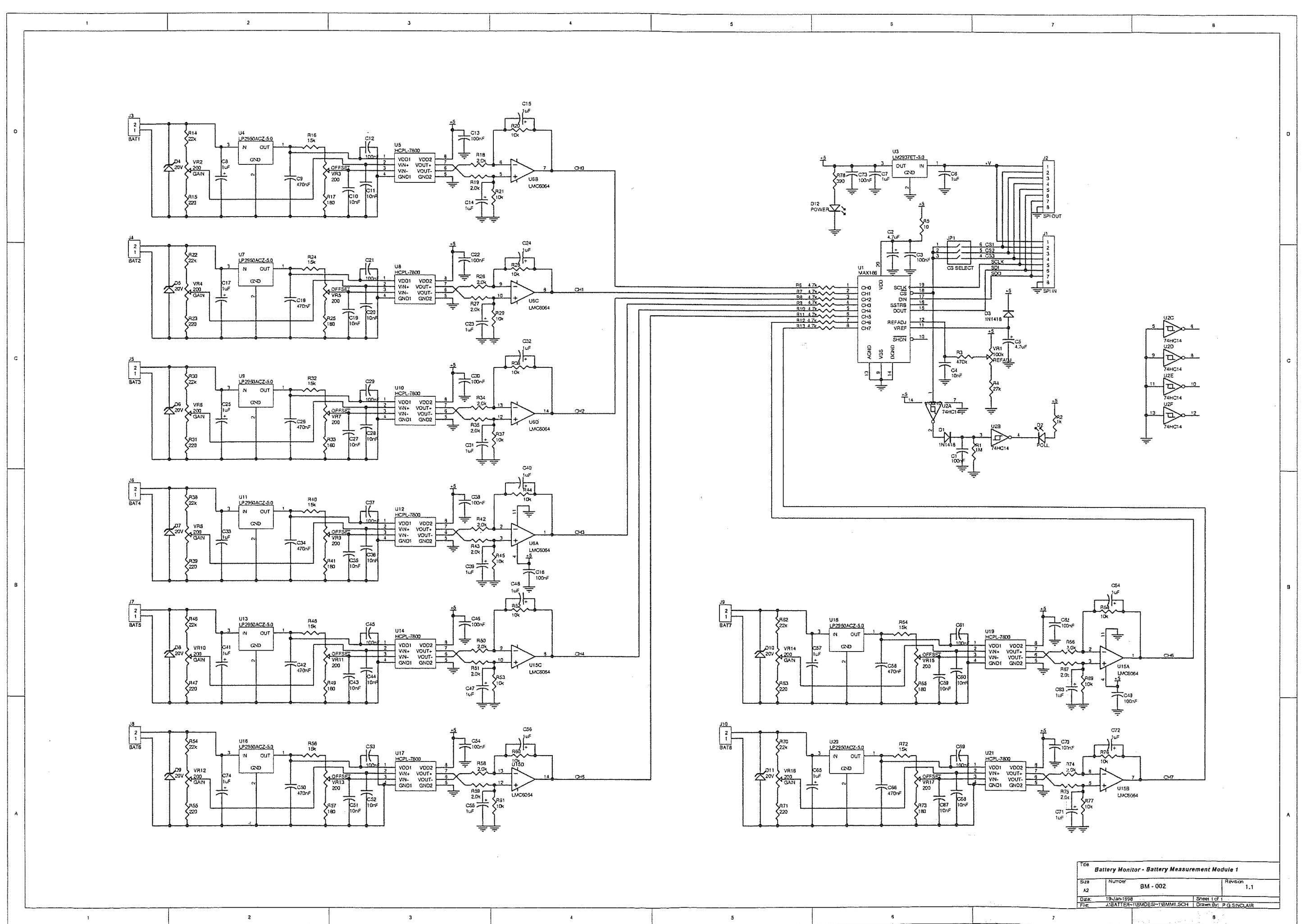


B2. Battery Measurement Module 2

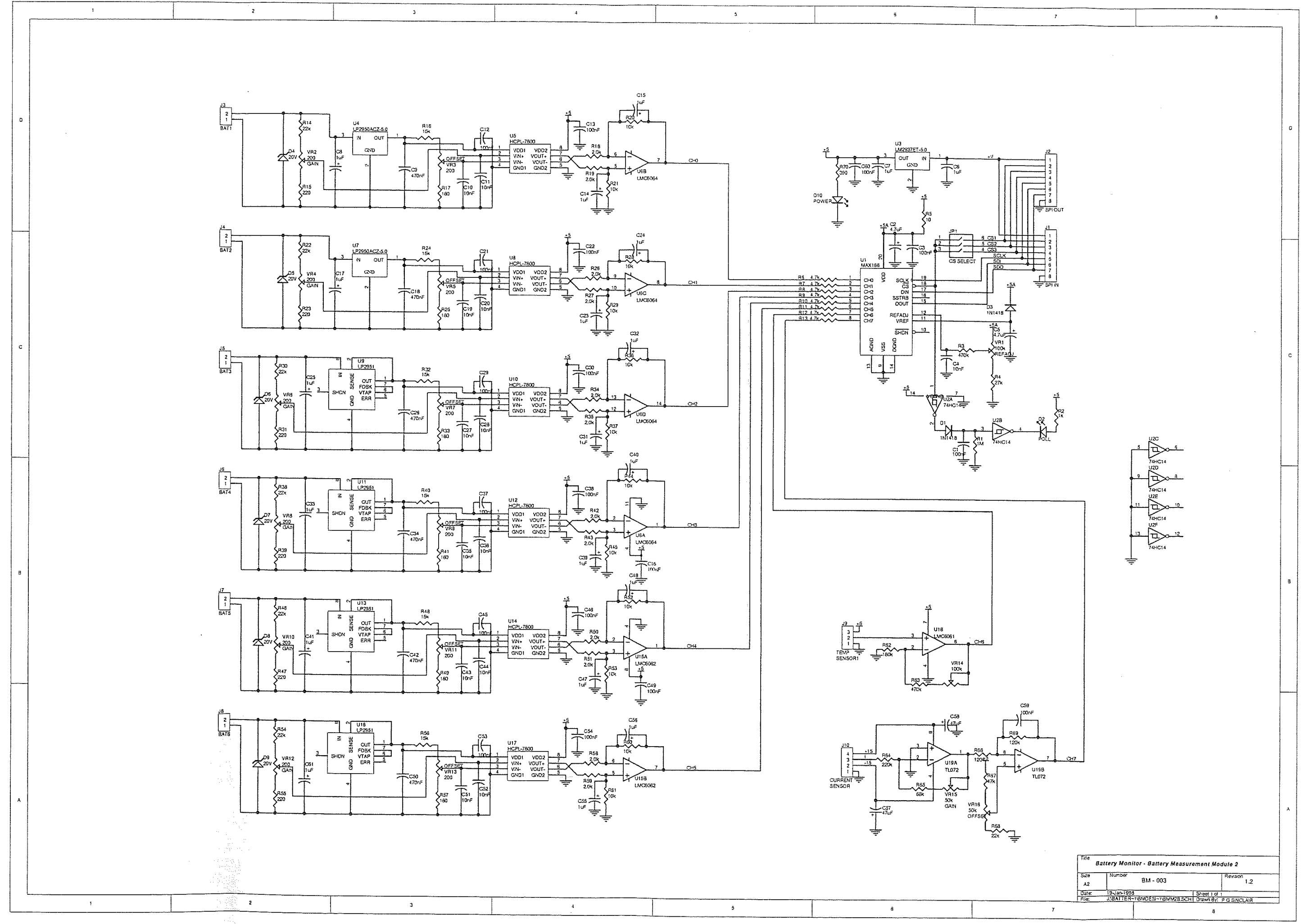


B3. Battery Measurement Module 3

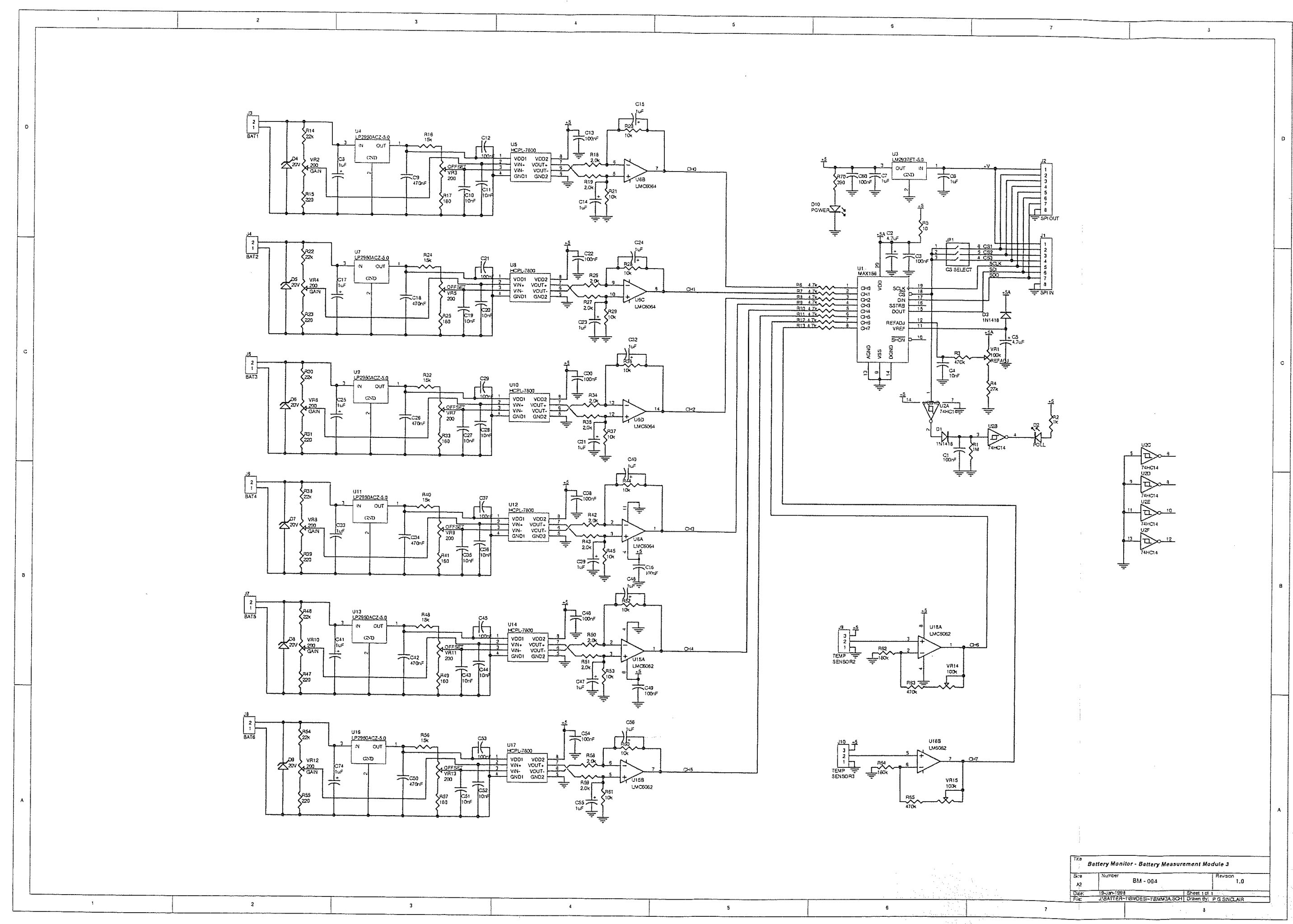




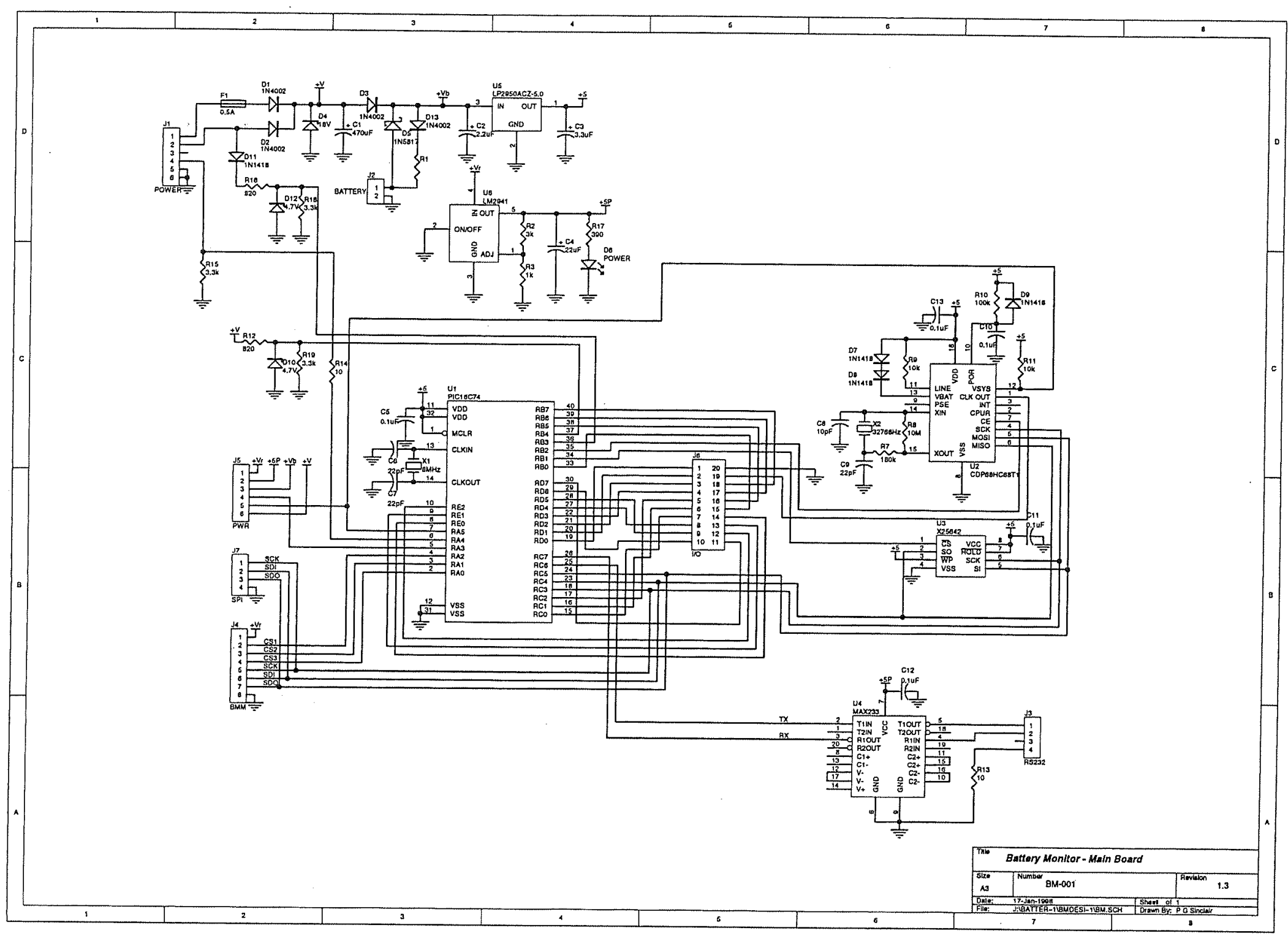

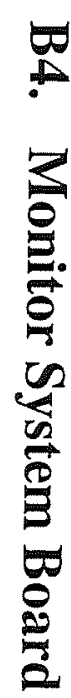




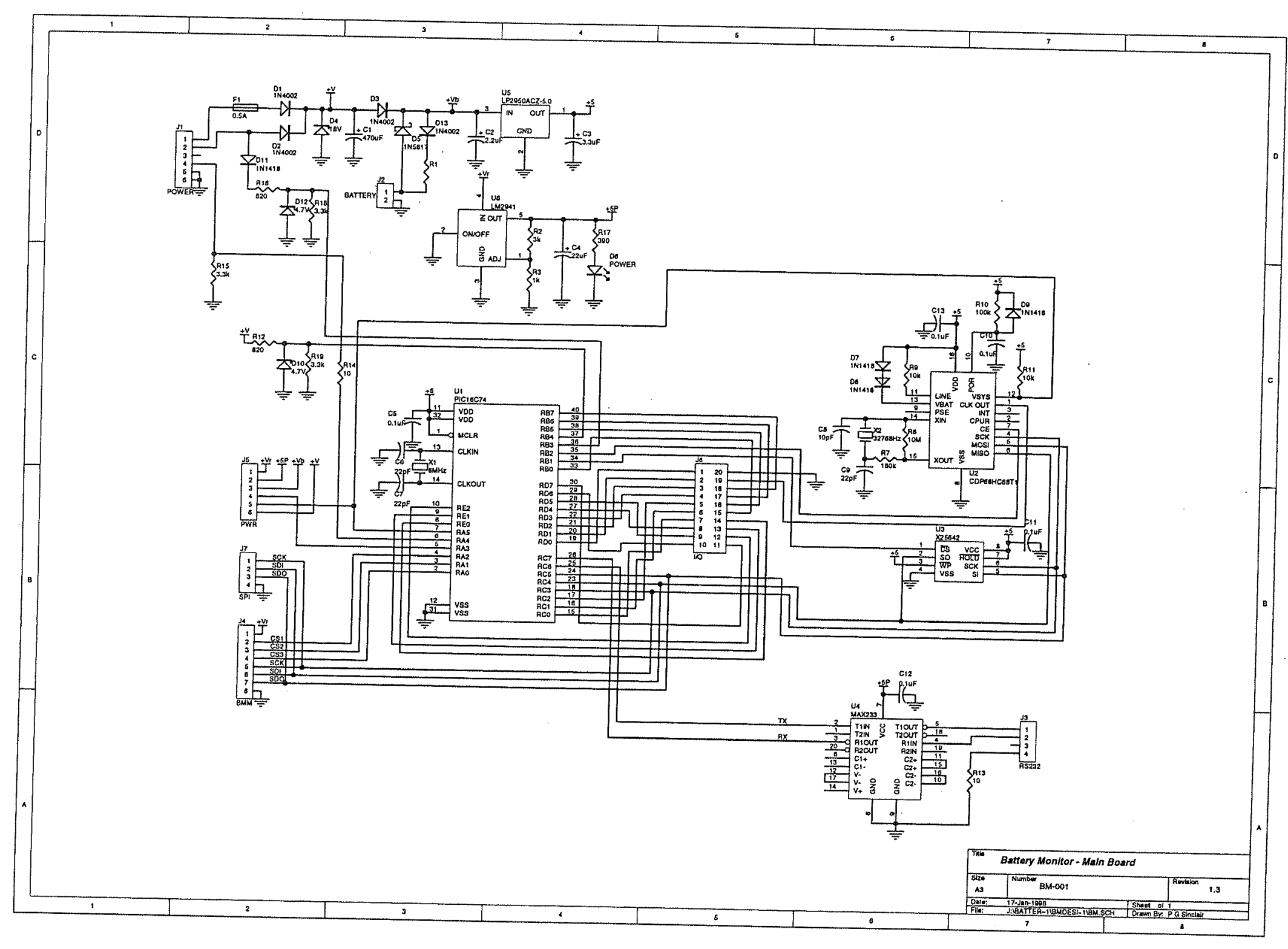




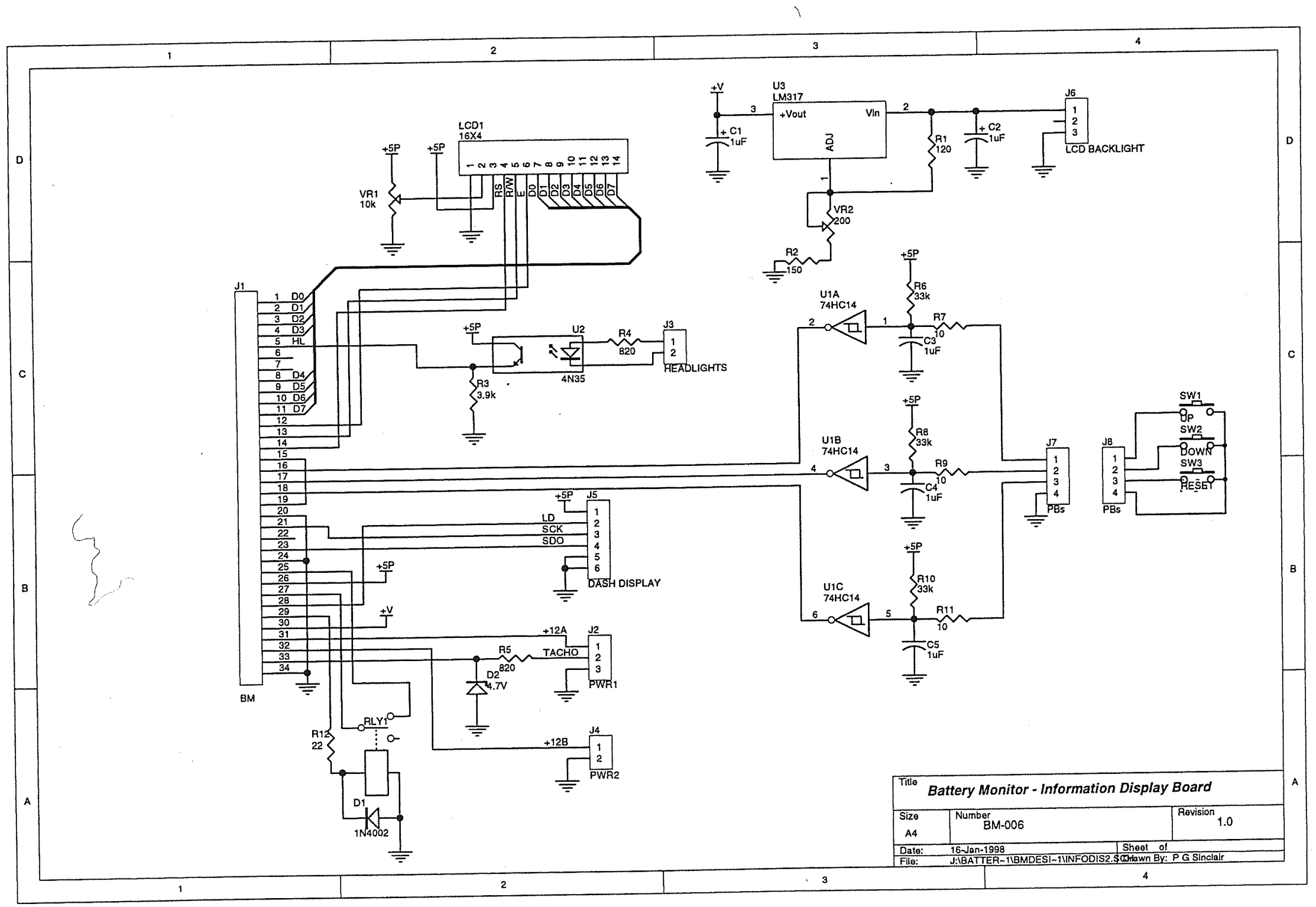

朵

3 


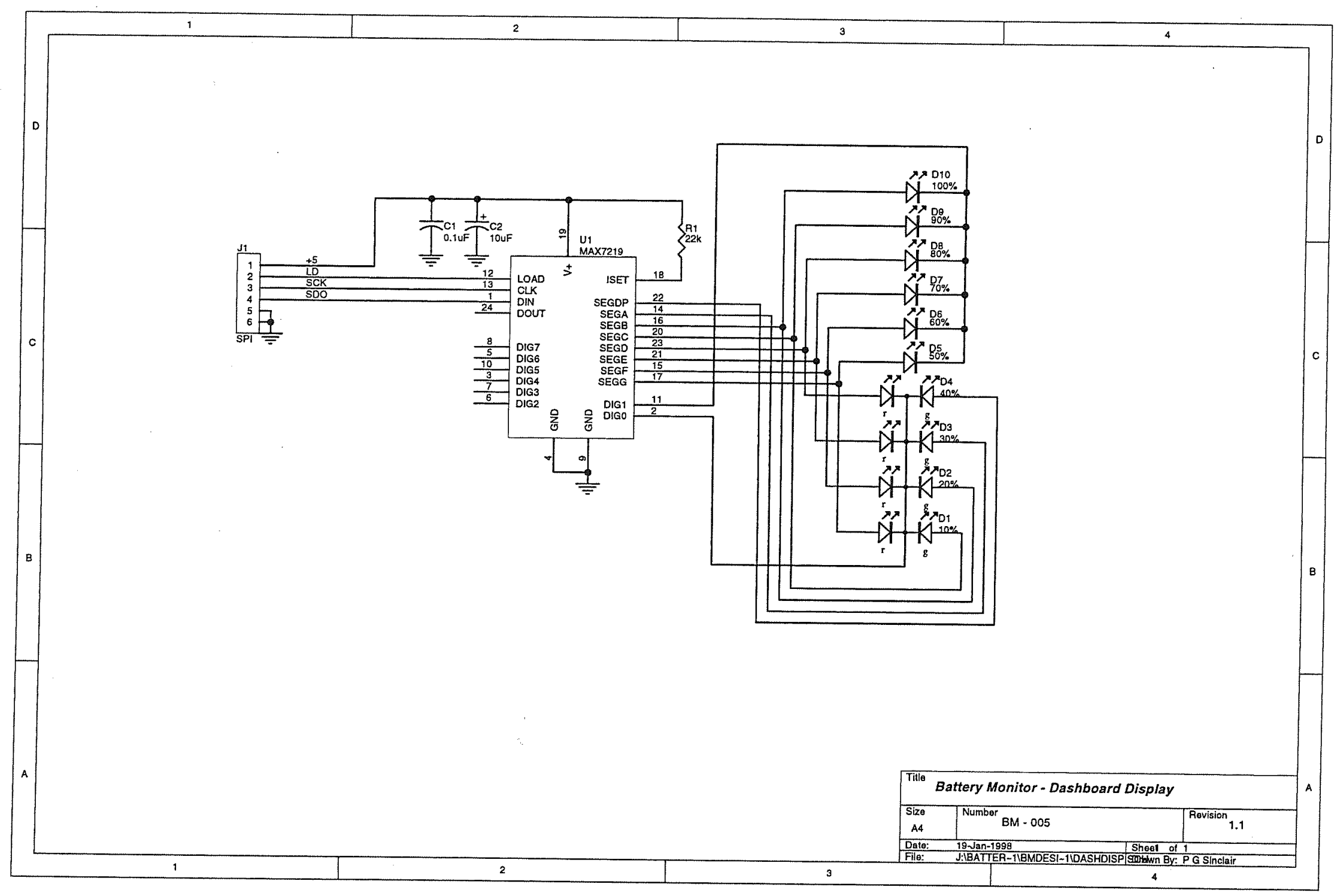

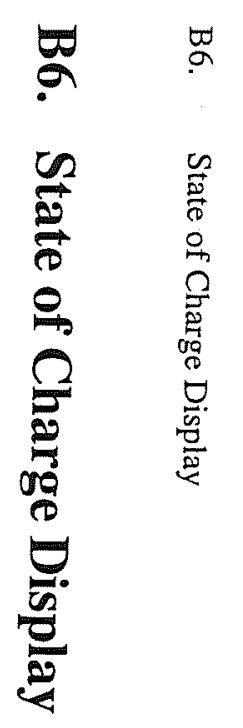




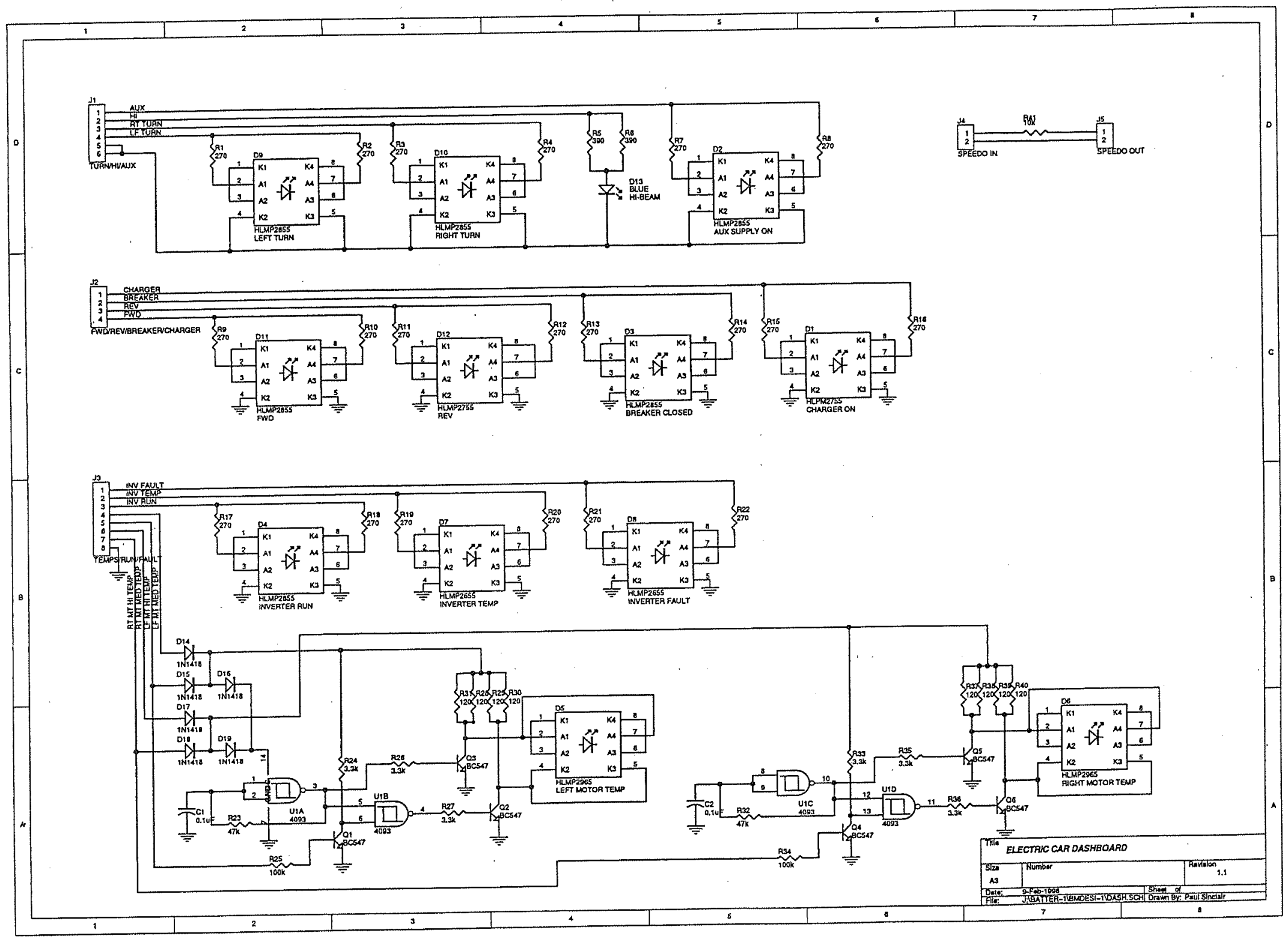

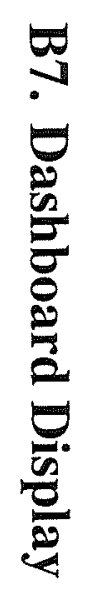




\section{Appendix C. PCB Artwork}

C1. Battery Measurement Module 1

C1.1 Top Layer
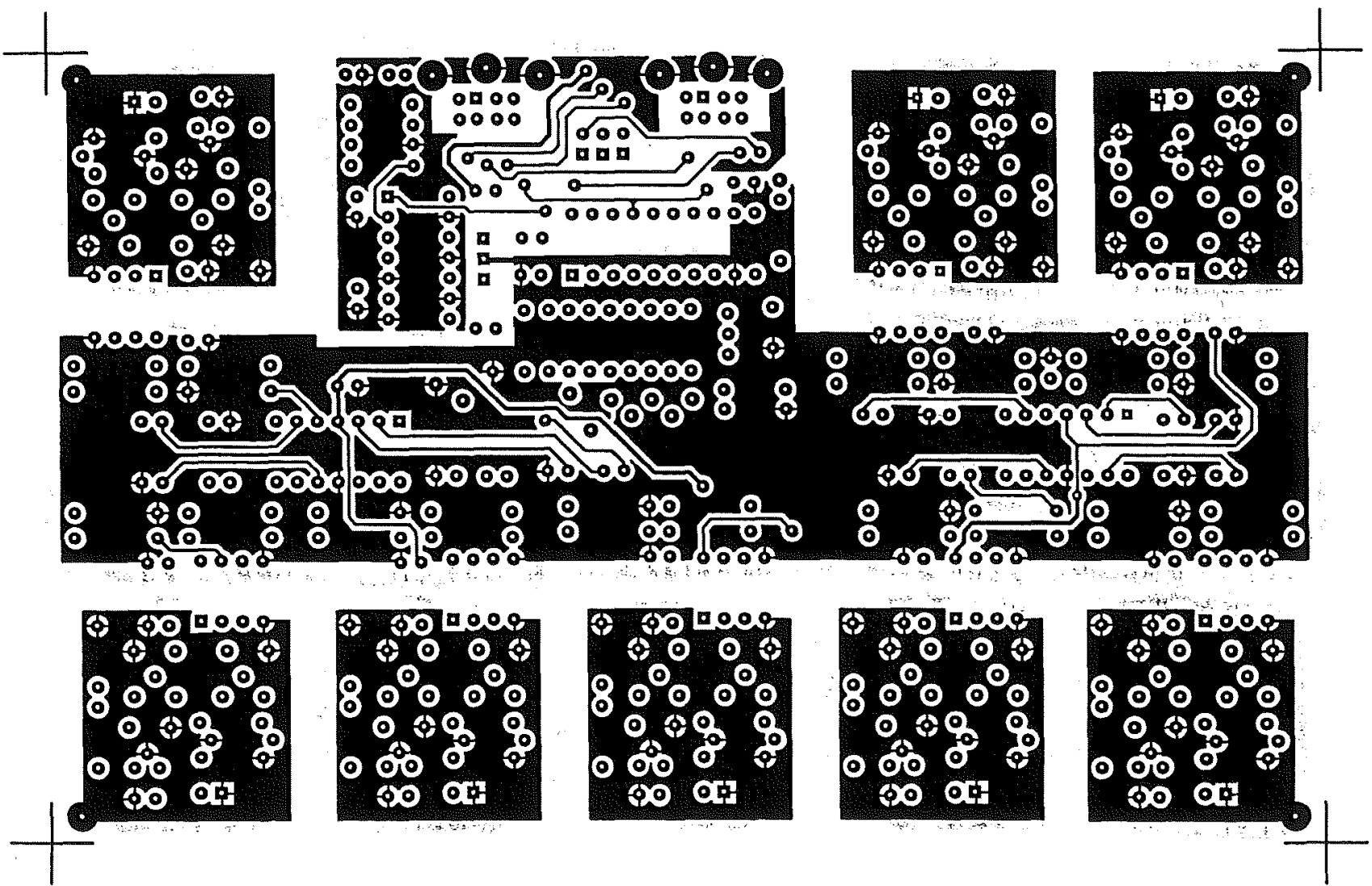


\section{C1.2 Bottom Layer}

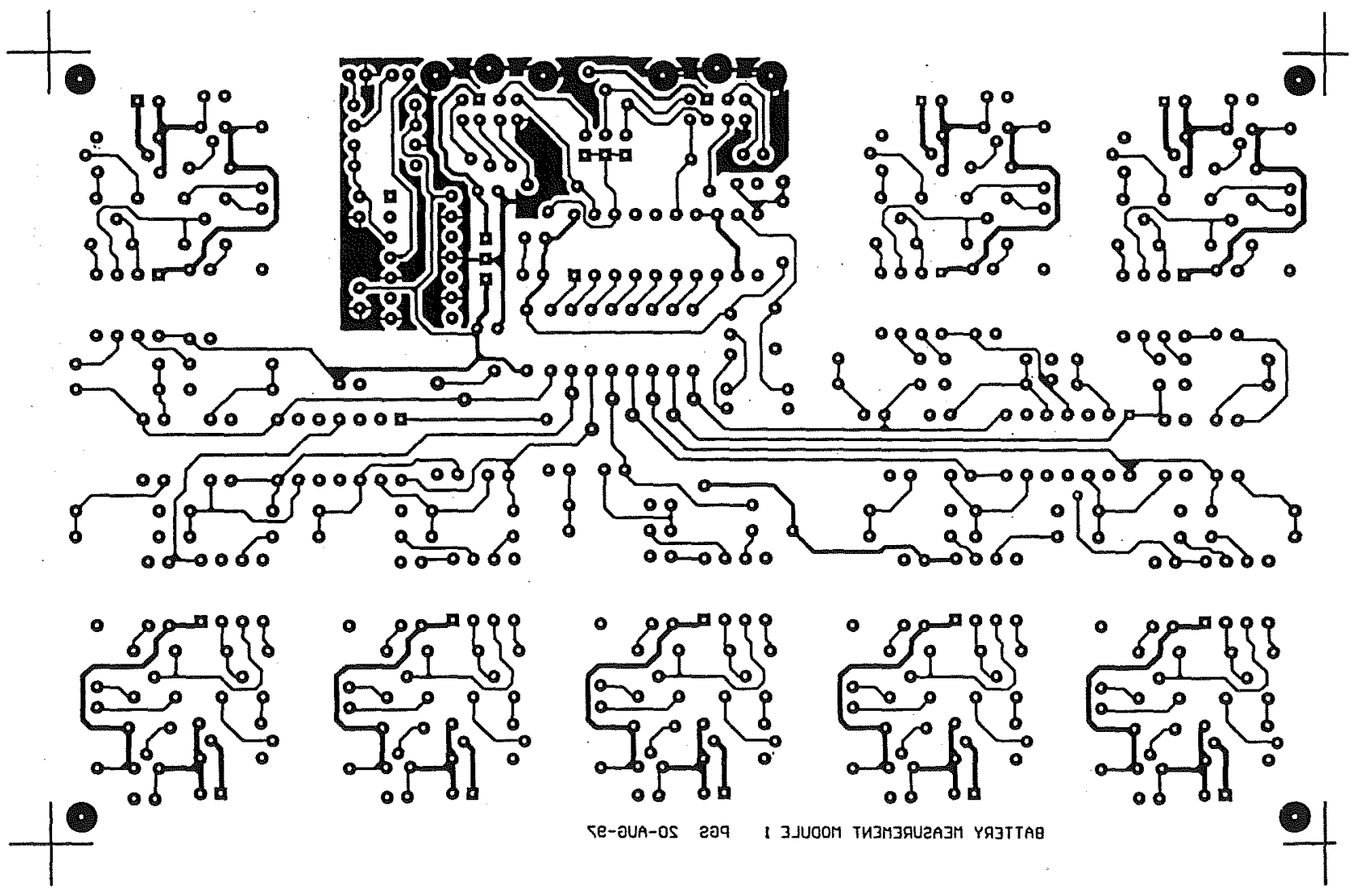




\section{C1.3 Component Overlay}

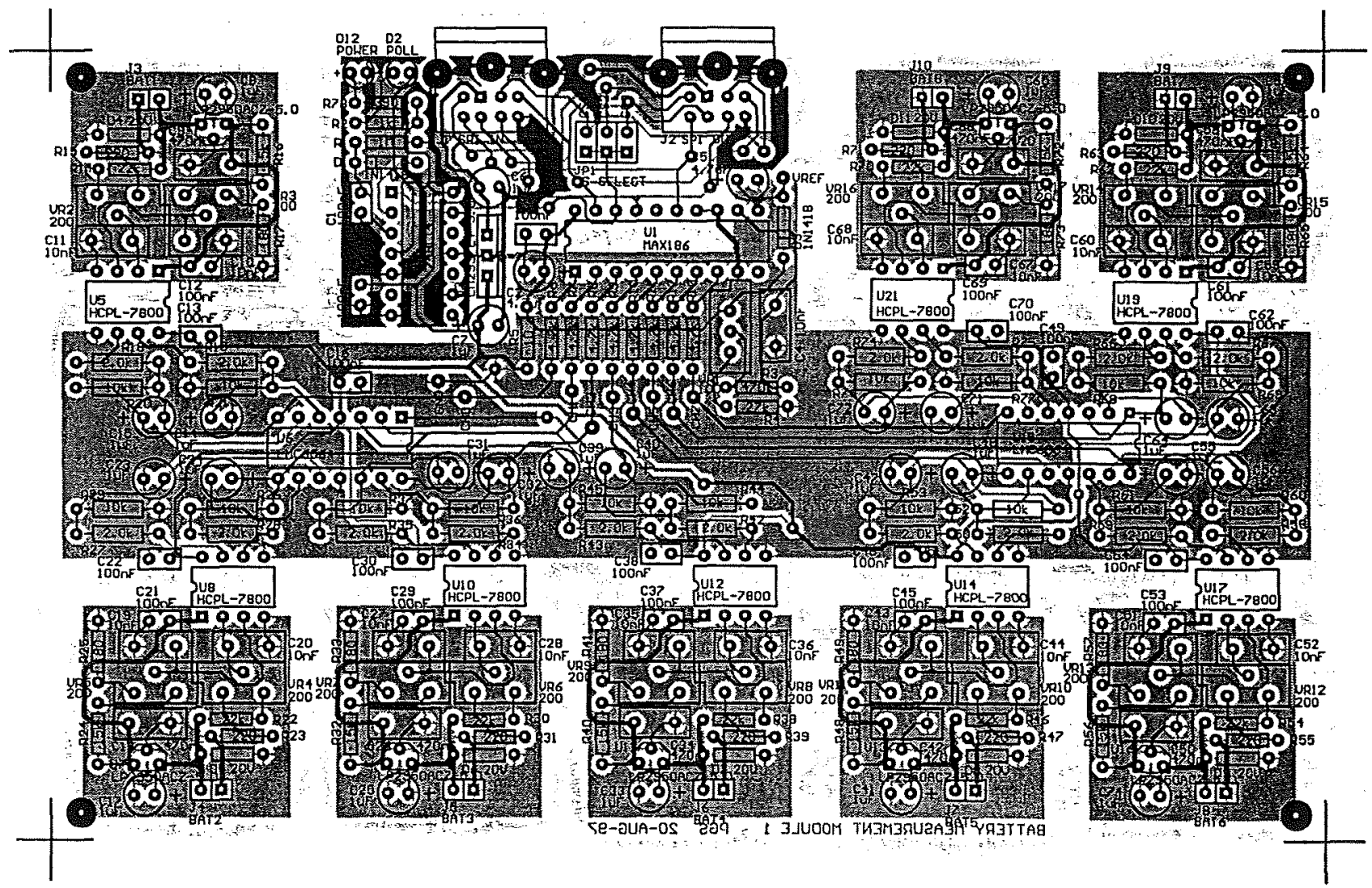


C2. Battery Measurement Module 2

\section{C2.1 Top Layer}
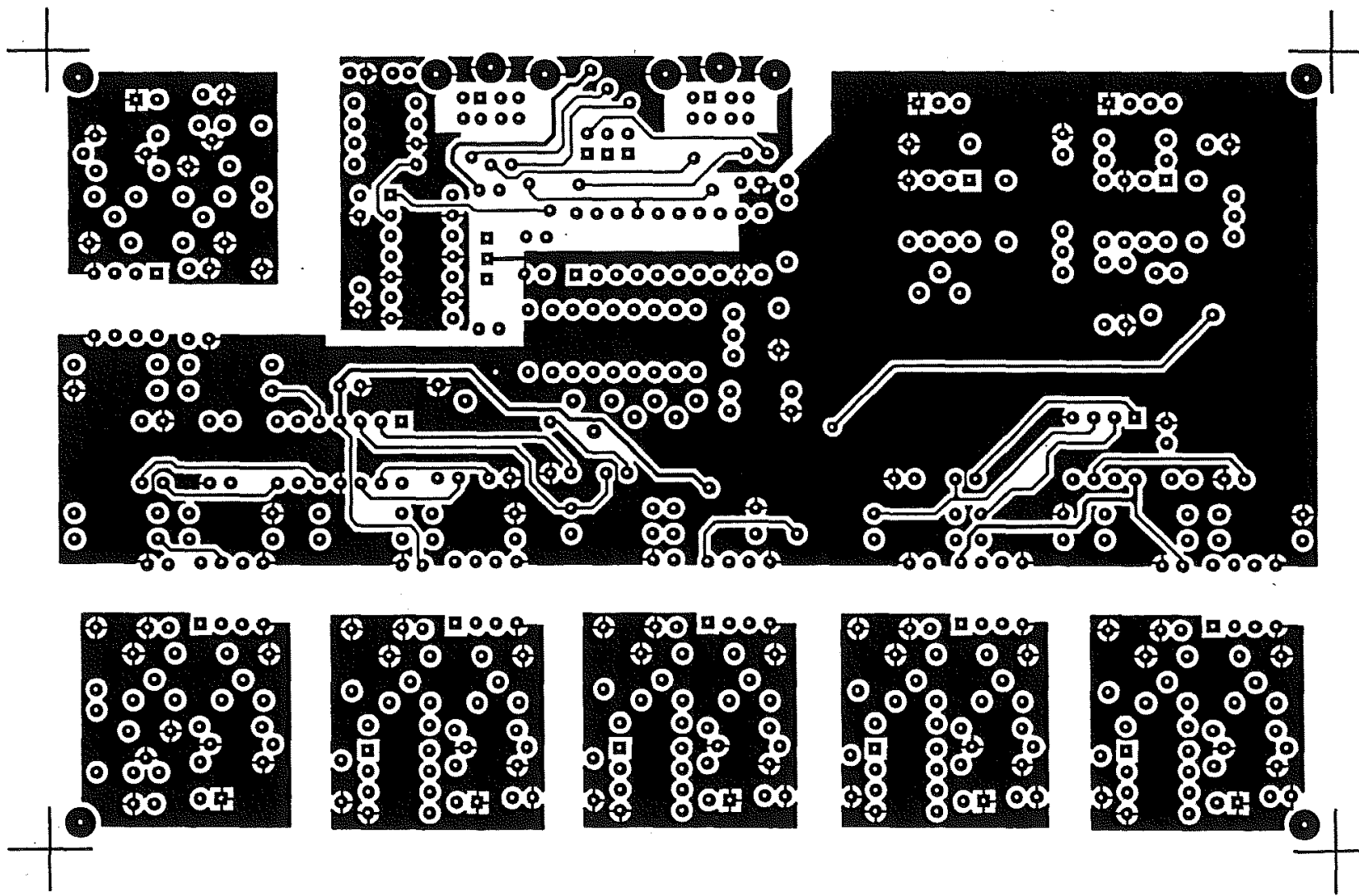


\section{C2.2 Bottom Layer}
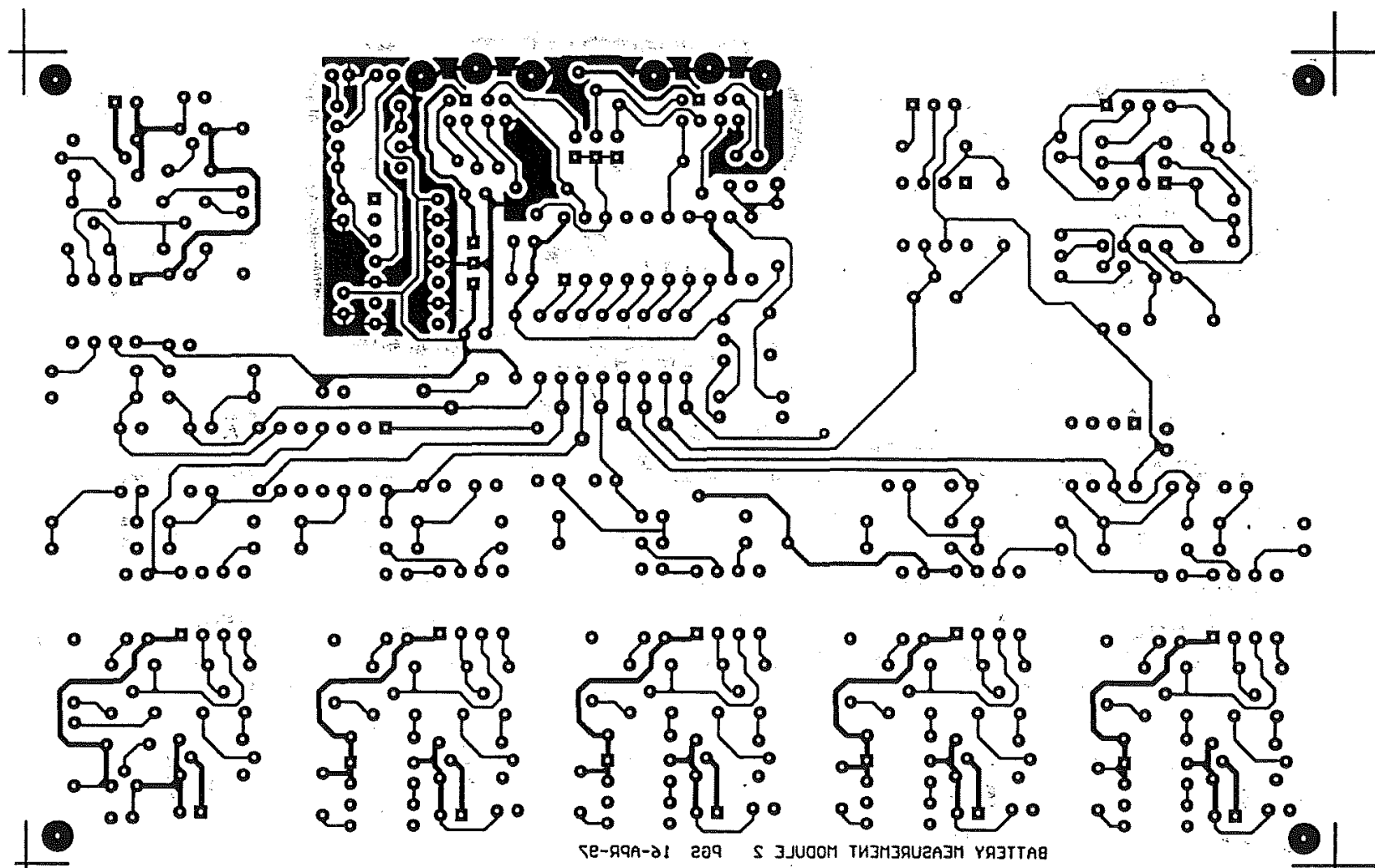

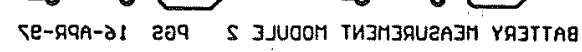

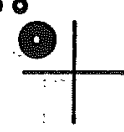




\section{C2.3 Component Overlay}

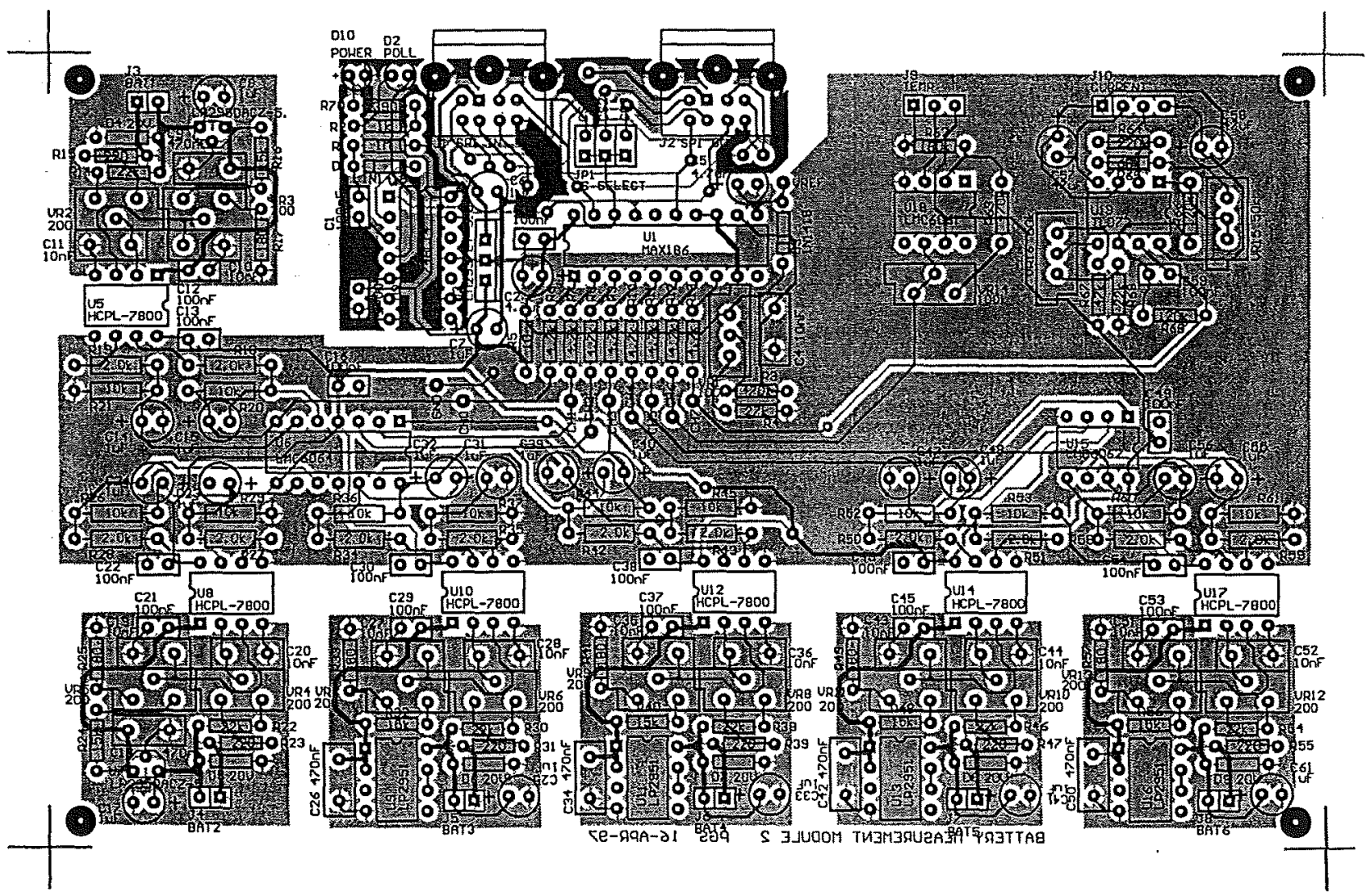


C3. Battery Measurement Module 3

C3.1 Top Layer
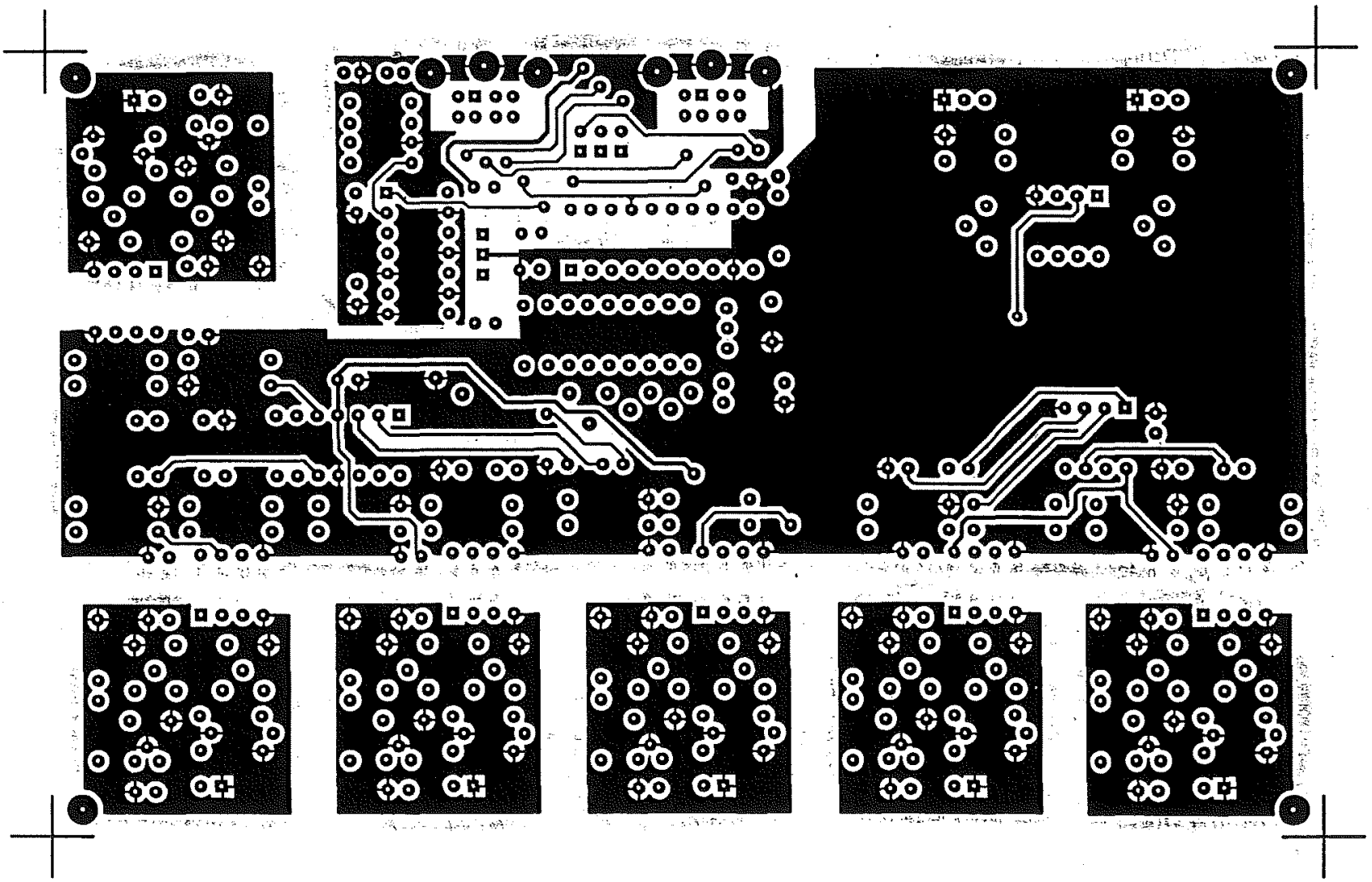


\section{C3.2 Bottom Layer}

10
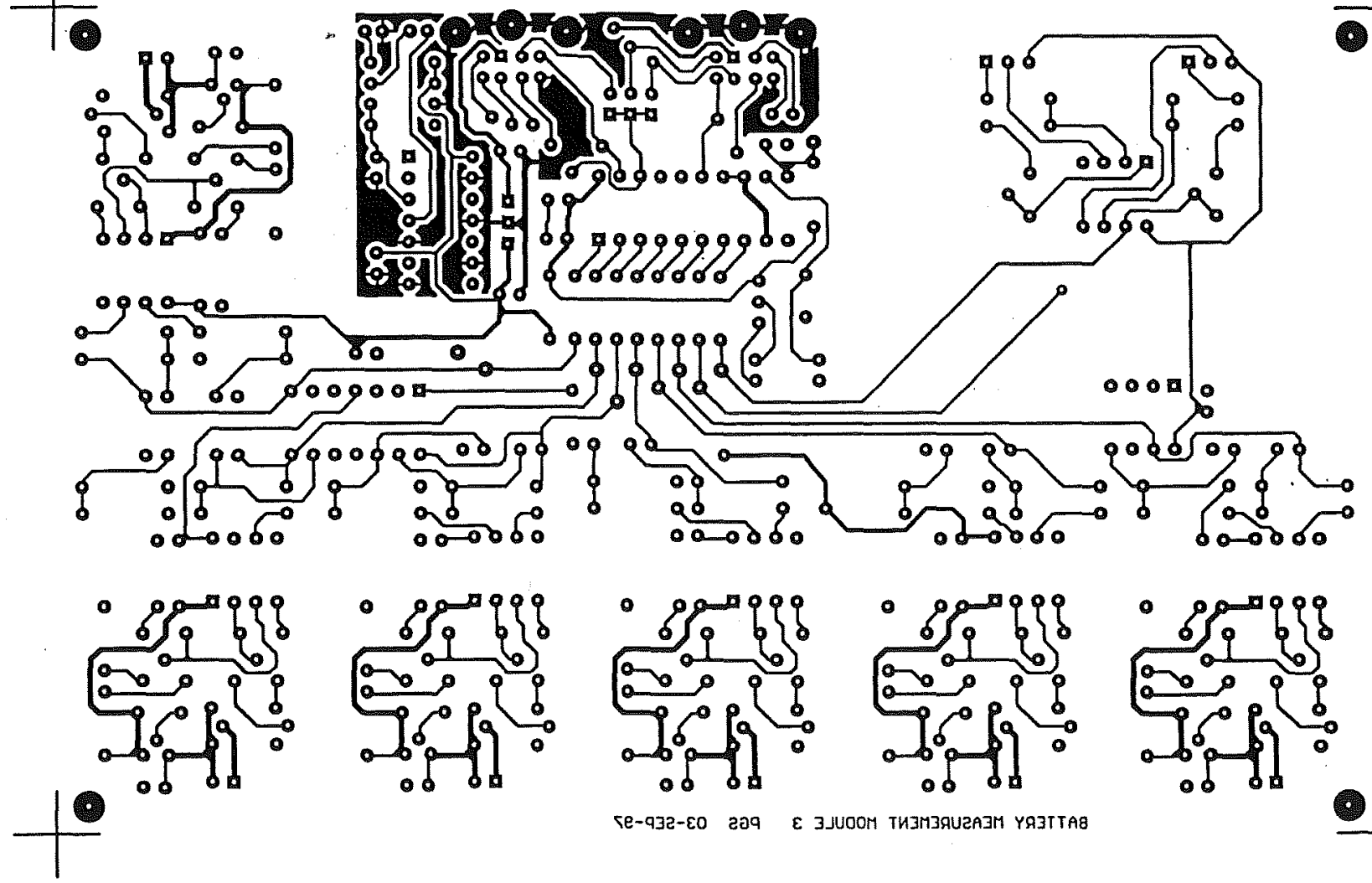

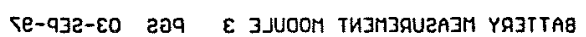




\section{C3.3 Component Overlay}

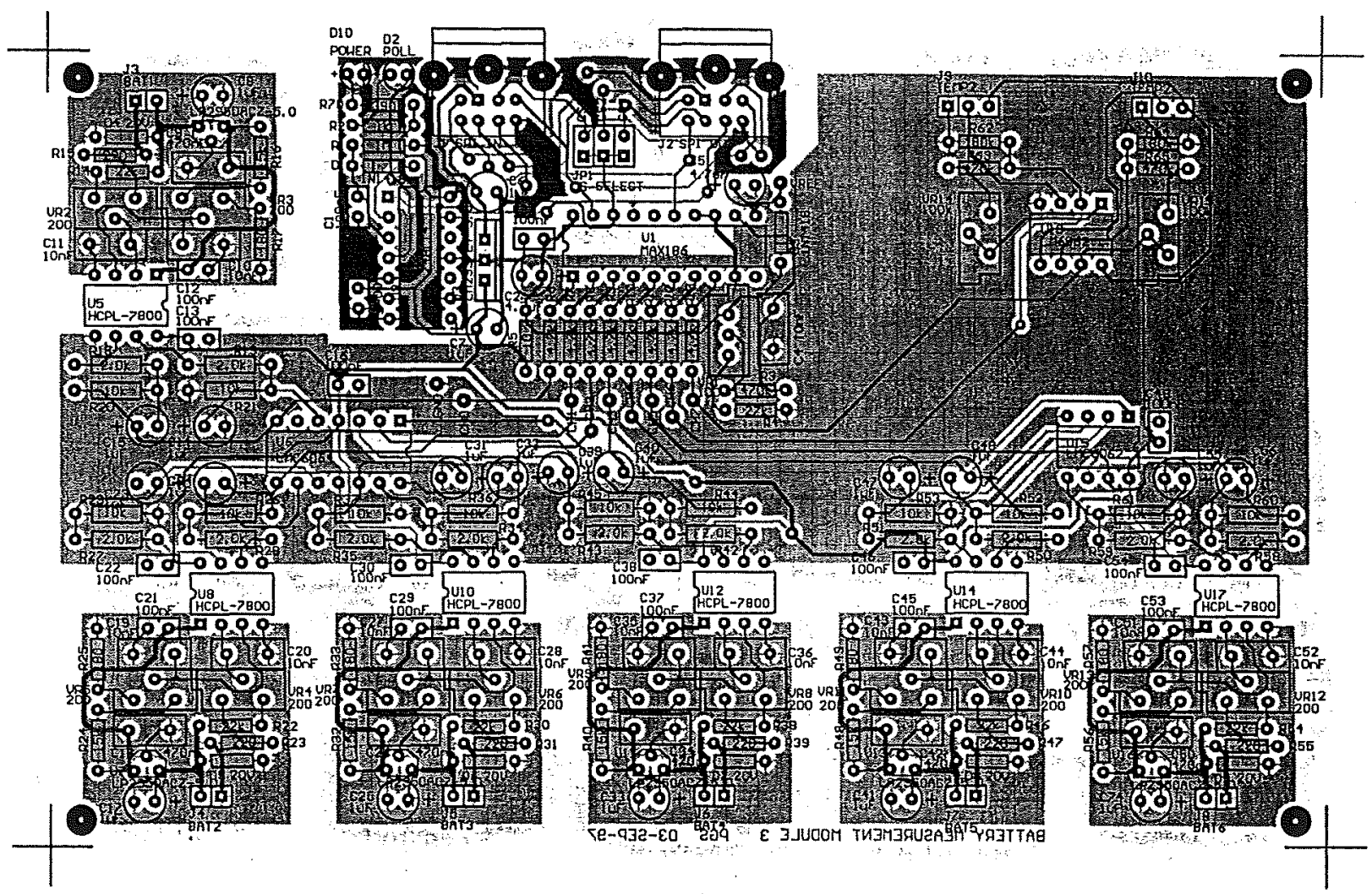




\section{C4. Monitor System Board}

\section{C4.1 Top Layer}

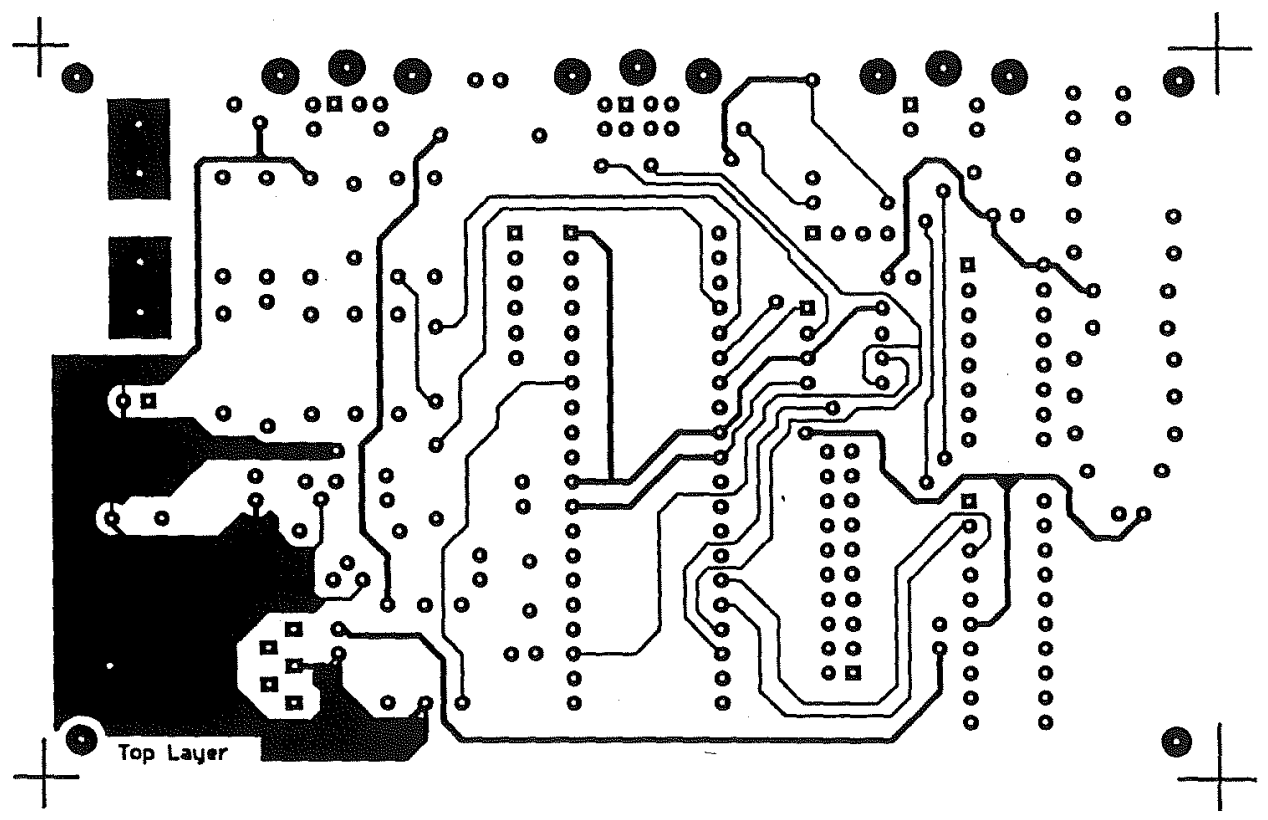

\section{C4.2 Bottom Layer}

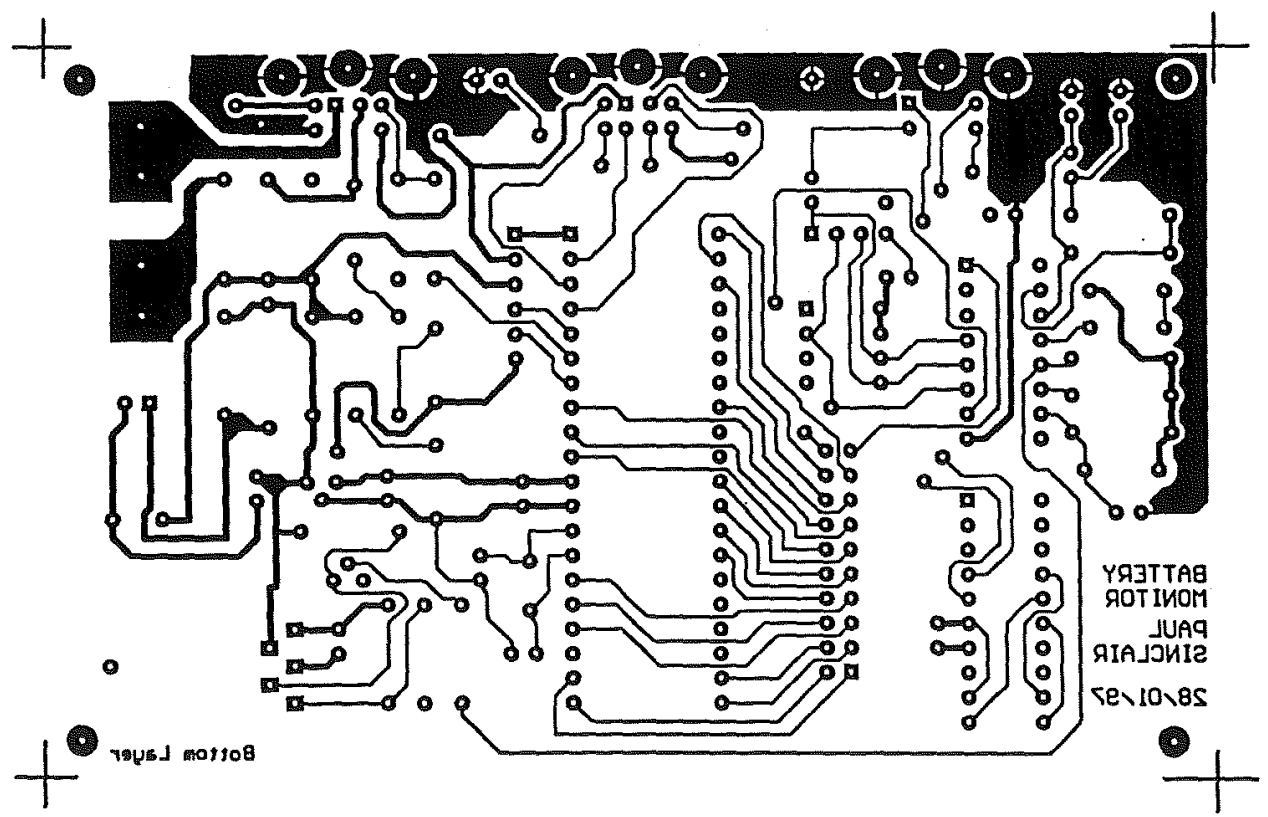




\section{C4.3 Component Overlay}

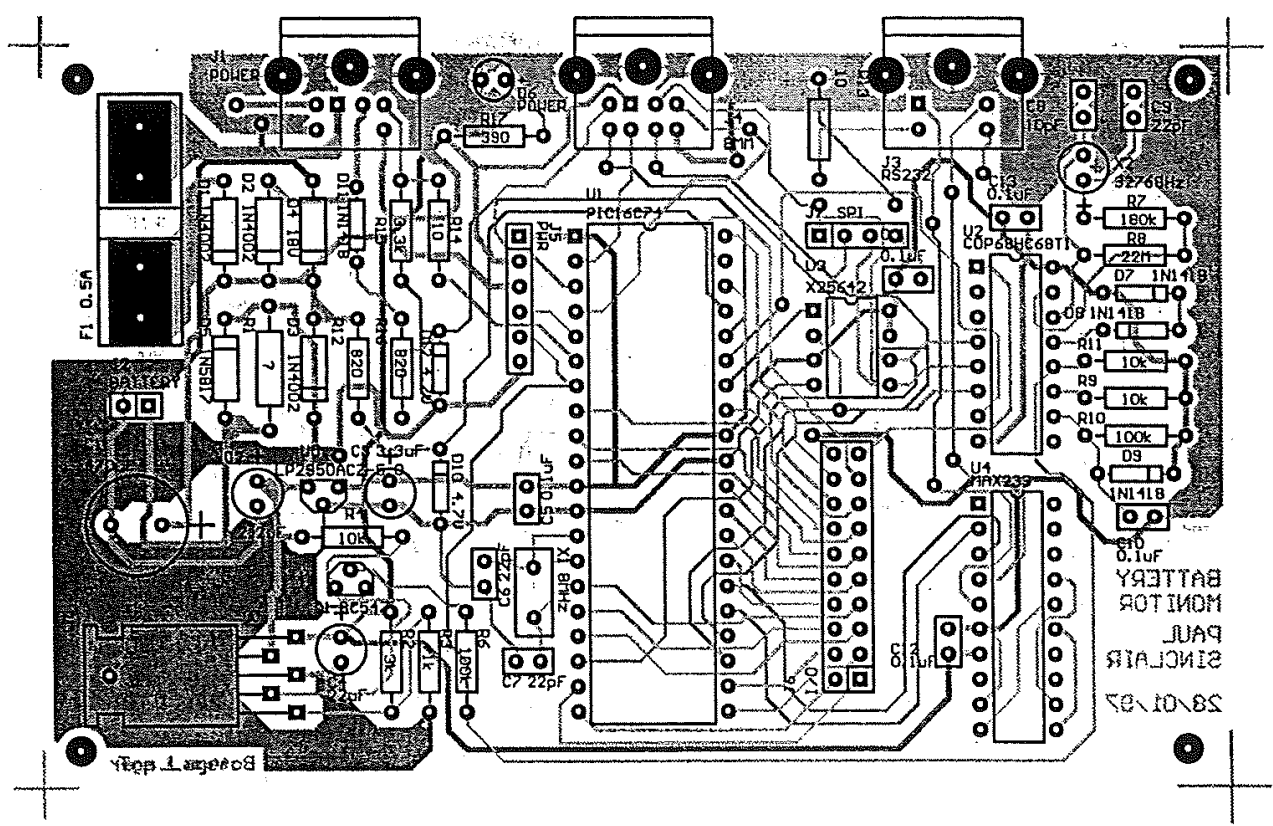




\section{C5. LCD Display Board}

\section{C5.1 Top Layer}

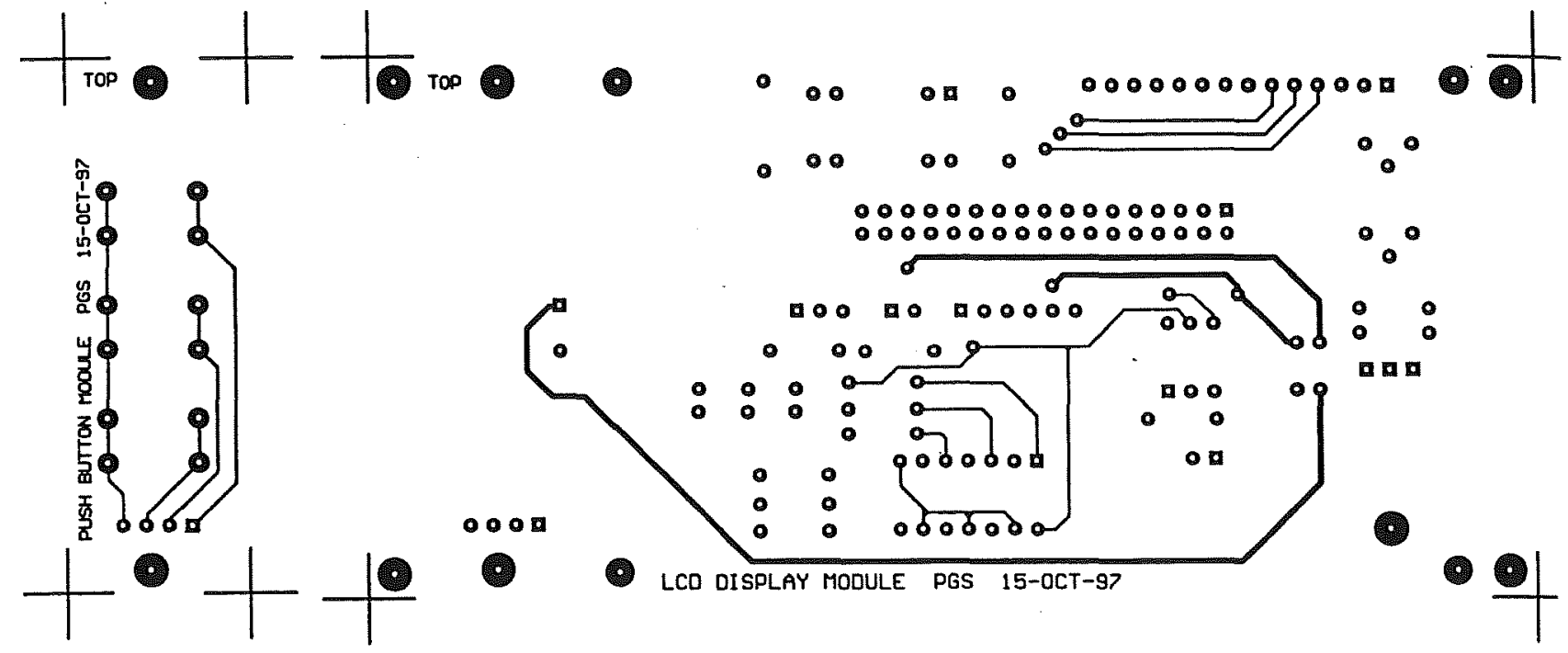

\section{C5.2 Bottom Layer}

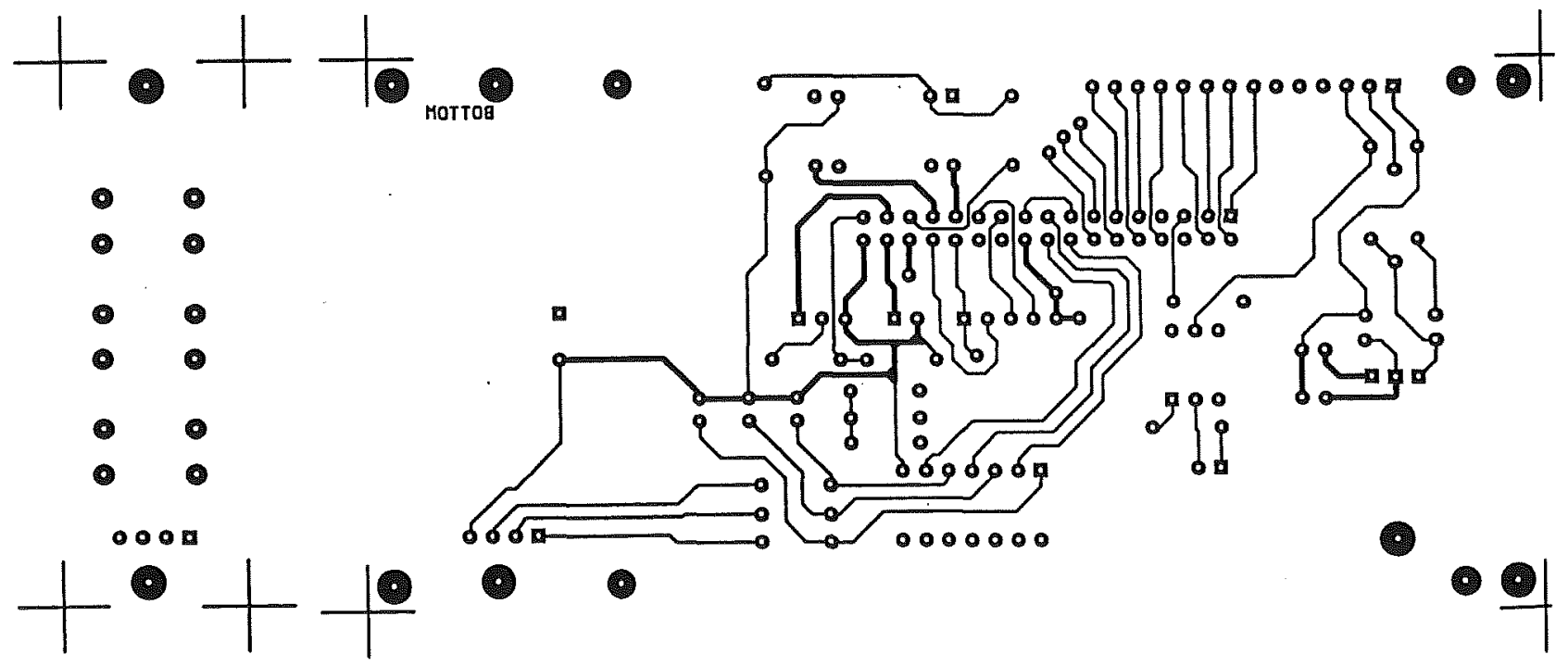




\section{C5.3 Component Overlay}

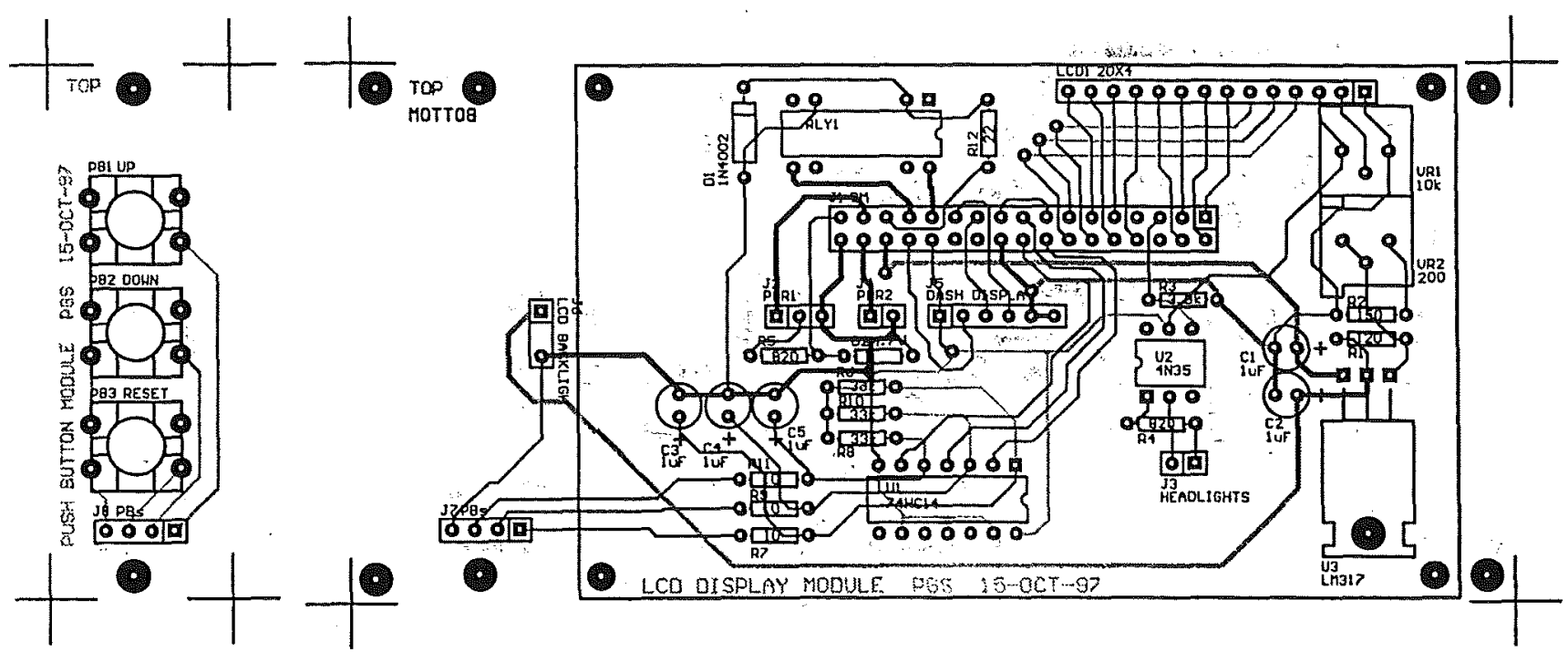




\section{C6. State of Charge Display}

\section{C6.1 Top Layer}

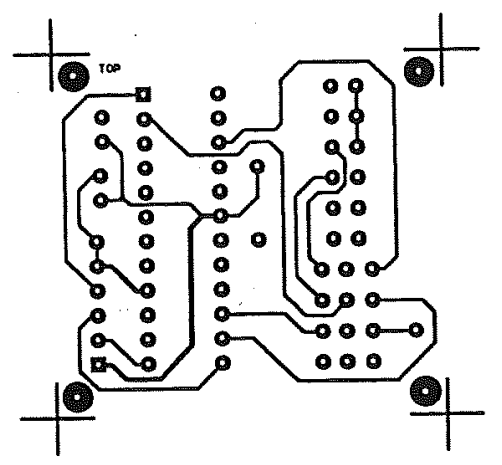

\section{C6.2 Bottom Layer}

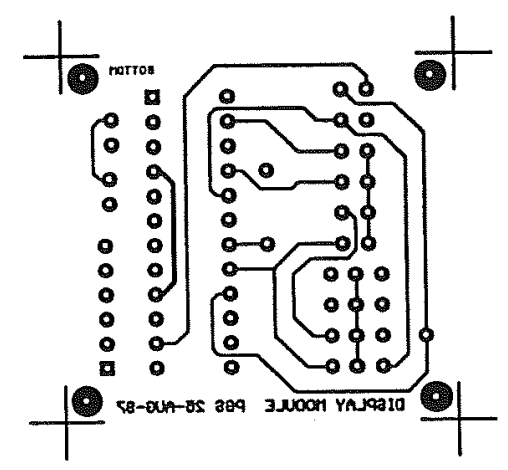

\section{C6.3 Component Overlay}

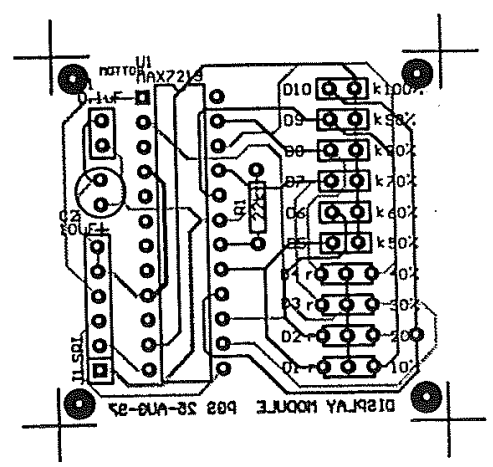




\section{C7. Dashboard Display}

C7.1 Top Layer

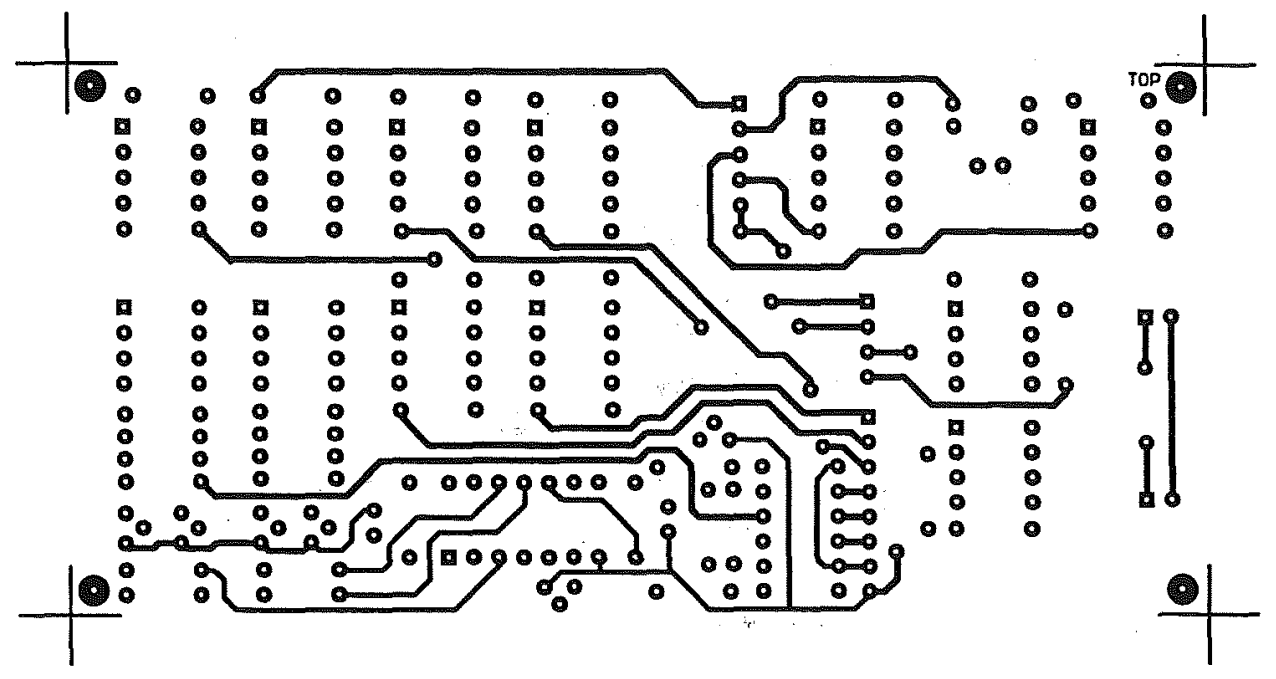

\section{C7.2 Bottom Layer}

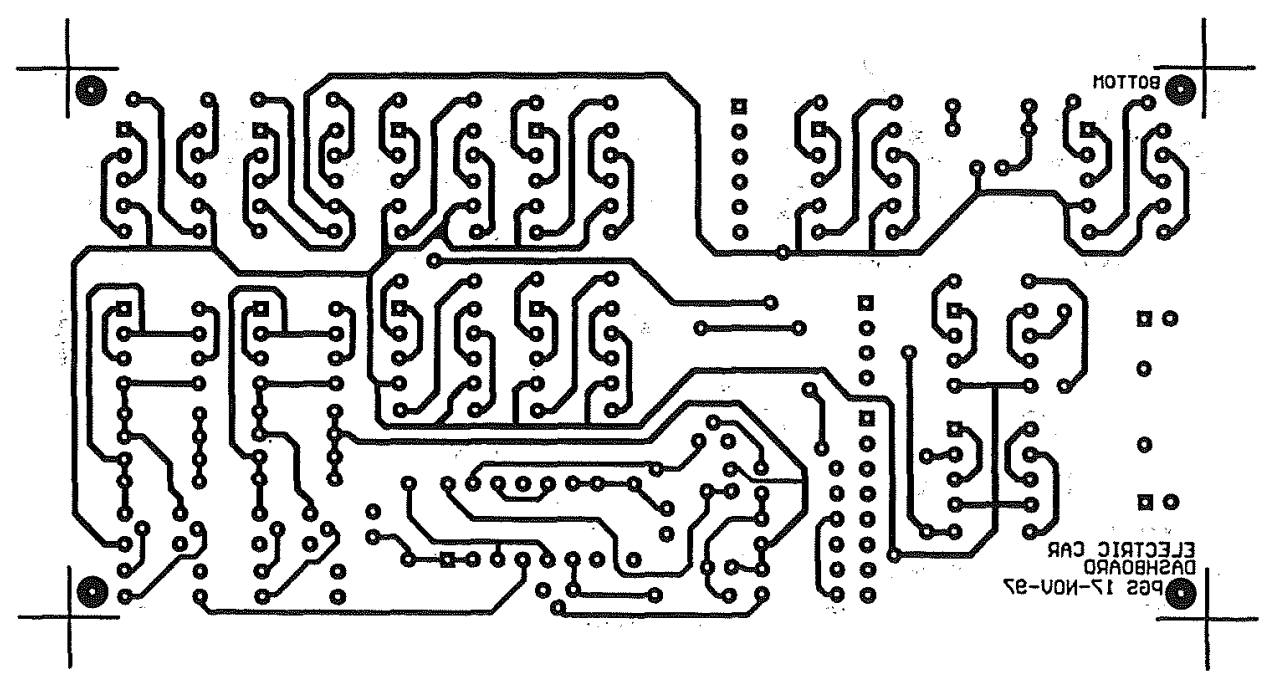




\section{C7.3 Component Overlay}

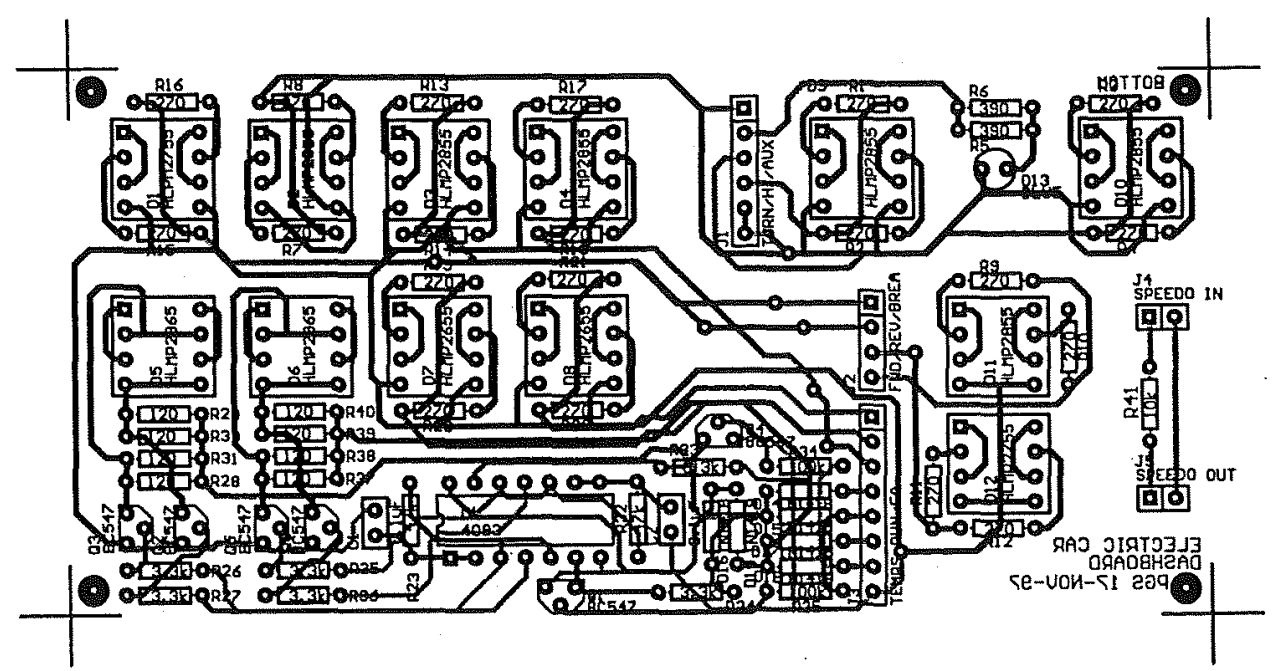




\section{Appendix D. Data Sheets}

\section{D1. Dryfit A200/A300 Lead-Acid Battery Specifications}

\section{maintenance-free lead acid accumulators Q}

\section{Solid advantages point-by-point:}

\begin{tabular}{|c|c|}
\hline $\begin{array}{l}\text { Maintenance-free } \\
\text { end sealed } \\
\text {. }\end{array}$ & $\begin{array}{l}\text { Noeds no maintenance whalsoever throughout its life. } \\
\text { Each cell is sealed by a valve preventing penetration by } \\
\text { air-borne oxygen. Over.pressure in the cells (e.g.. } \\
\text { through over-charging) unseats the valve so letting out } \\
\text { the excess independent pressure; the valve then closes } \\
\text { again. }\end{array}$ \\
\hline $\begin{array}{l}\text { Deep-discharge } \\
\text { resistant } \\
\quad\end{array}$ & $\begin{array}{l}\text { dryfit batteries survive deep-discharging without } \\
\text { suffering damage. Even when discharged and remaining } \\
\text { connected to a live consumer for } 4 \text { weeks, they pecover } \\
\text { to } 80 \% \text { their capacity after } 48 \text { hours charging. } \\
100 \% \text { is reached after a few cycles. }\end{array}$ \\
\hline $\begin{array}{l}\text { Extremely low } \\
\text { self-dischanging }\end{array}$ & $\begin{array}{l}\text { Less than } 0.1 \% \text { of the rated capacity per day at }+20^{\circ} \mathrm{C} \\
\text { ambient temperature means to re-charging even after } \\
\text { up to } 2 \text { years storage. }\end{array}$ \\
\hline $\begin{array}{l}\text { Cyclic resistance } \\
\quad \therefore \quad\end{array}$ & $\begin{array}{l}\text { Special measures relating to electrolyte production make } \\
\text { A } 200 \text { version dryfit battertes partlcularty cyclic risistant. } \\
\text { At } 100 \% \text { discharge (up to discharge cut-ofl voltage of } \\
1.75 \text { Volts/cell) more than } 200 \text { cycles cian be drawn. } \\
\text { Considerably more cycles are possible with partial } \\
\text { dlscharges. }\end{array}$ \\
\hline Long-Iffe & $\begin{array}{l}\text { the life is } 4-5 \text { years, } \\
\% \text { of the rated } \\
334) \text {. }\end{array}$ \\
\hline Wide temperature range & $\begin{array}{l}\text { briefly excreded). } \\
\text { rature condifions, } \\
\text { tions. }\end{array}$ \\
\hline $\begin{array}{l}\text { High load capacity, } \\
\text { all-round use }\end{array}$ & $\begin{array}{l}\text { and connector design gives good high- } \\
\text { propenties. Excellently sulted for op bration } \\
\text { ne conditions due to high resistancd to } \\
\text { io larger types (from } 24 \text { Ah) are sultaple for } \\
\text { ibustion motors. }\end{array}$ \\
\hline $\begin{array}{l}\text { Simplo charging } \\
\text { method }\end{array}$ & $\begin{array}{l}\text { Just one charging voltage for cycllc and continupus } \\
\text { charglng modes. No current limiter needed as cinarging } \\
\text { current is regulated by the battery. Constant chitging } \\
\text { voltage at }+20^{\circ} \mathrm{C} \text { room temperature is } 23 \text { Volts'cell. } \\
\text { For cyclle use we recommend a charging voltage of } \\
2.35 \text { V/cell. }\end{array}$ \\
\hline Wide capacity range & $\begin{array}{l}\text { ries are avallable in differe } \\
12 \text { V/200 Ah; for the indus }\end{array}$ \\
\hline Non dangerous goods & fit A 200/A 300 \\
\hline $\begin{array}{c}\text { Approvals } \\
\therefore \quad\end{array}$ & $\begin{array}{l}\text { dryfit A } 200 / \text { A } 300 \text { batteries are conform in construction } \\
\text { and performance with the standards: DIN 43534, } \\
\text { DIN } 43539 \text { part } 1 \text { and } 5, \text { IEC } 1056 \text { part } 1 \text { and } 2 \text {, UL and } \\
\text { selected types VdS. }\end{array}$ \\
\hline
\end{tabular}

Tested and found to be good!

\section{Sonnenschein}

dryfit batteries

comply with the following international standards:

dryfit A 200

DIN 43534 "Maintenancefree" seated rechargeable batteries with golled electrotyte.

DIN 43599 Part 5 Tests . Mantenance-free sealed rechargeable batteriss wis appoves vis approvals: Selected types approved by VdS ffederation of German speclalist. Insurers).

DIN 57510 NDE 0510 Rechargeable batterles and battery systems. stationary batteries. NATO - Selected types tested and approved according to guldellnes for military supply standards.

dryfit A 200/A 300

DINEN 50 014/ VDE 0170/0171 Parl 1/5.78 General specificattons. OIN 57 833/VDE 0833 Part 1 Danger waming equipment for fire, assaut robbery and burglary.

UL recognition File MH 12547. GL Germanischer Lloyd No. $99450 \mathrm{HH}$ $99451 \mathrm{HH}$.
$89452 \mathrm{HH}$. Installation of dryfit batteries in rooms or cabinets: the recommendations of the VDE 05TO Part 2 and 7 must be taken into 
Performance Features and Technical Specifications Fig. 1 shows the loada 2 Volt battery, e.g. a 24 An battery is dis. charged with $12 \mathrm{~A}$. $24 \%=1.2 \mathrm{~A}$.

$12 \mathrm{~A}$ a*: $\mathrm{A}: \mathrm{l}, \mathrm{s}, 2=10 \mathrm{Kl}$

The voltage curve and dlscharge time for this example can be seen from example can be
Fig. 1 at $10 \times l_{20}$

Connections

for reliable contact

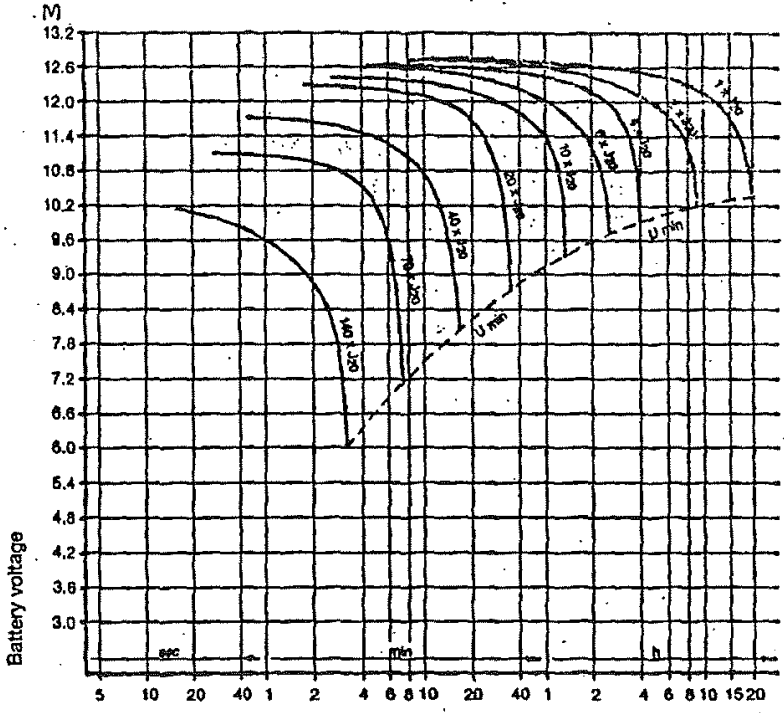

Fig. 1. Discharge time

$V_{m m}=$ Normal minimum permissible dlscharge voltage (gulde values)

Capacity in relation to the load (guldo values)

Fig. 2 shows the percentage of withdrawable discharge current value. The figures apply to now batteries. In cyclic and parallel standby operation development occurs. Winh high-current discharges the max. load values given in the Tabte on pages 4 and
V

Sitver-plated flat contact

with sotder lugs and

connecting tags for

4.8 mm and $6.3 \mathrm{~mm}$

$S$

Silver-plated flat contact

with solder lugs and

$U$

Silver-plated laterally offset flat contact

$\mathrm{K}$

Silver-plated contact rivel shaped

N

Silver-plated flat contact on narrow side of battery

A

Torminal plliar to

DIN 72311 made of lead.

For higher dlscharge

currents use only tapered connector

G

Drilled flat terminal, lead, Type $6 M \times 6 \mathrm{G} 5$ or $\mathrm{G} 6$,

Matching screw-on flat

connectors 4.8 or $6.3 \mathrm{~mm}$

are commercialty available.

$G=6 \mathrm{~mm}$ connection connecting tags for
$4.8 \mathrm{~mm}$ FASTON sockets

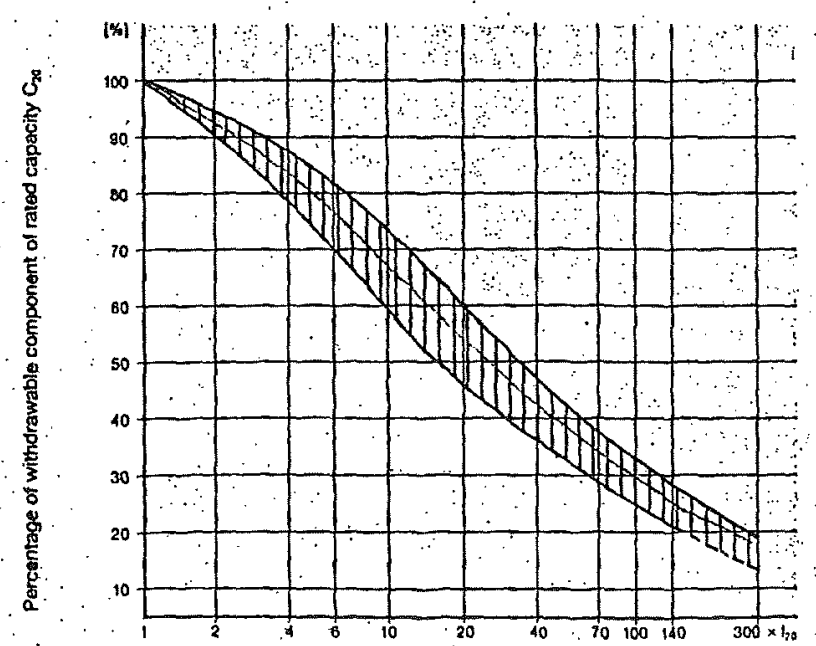

Fig. 2

Multiple of diseharge current $1 \%$ 


\section{D2. MAX186 8-Channel, Serial 12-Bit ADC}

19-0123; Rov. 3; 4/95

\section{General Description}

The MAX186/MAX188 are 12-bit data-acquisition systerns that combine an 8-channel multiplexer, high-bandwidth track/hold, and serial interface together with high conversion speed and ultra-low power consumption. The devices operate with a single $+5 \mathrm{~V}$ supply or dual $\pm 5 \mathrm{~V}$ supplies. The analog inputs are software configurable for unipolar/bipolar and single-ended/differential operalion.

The 4-wire serial interface directly connects to SPIm, QSPI'M and Microwire ${ }^{\mathrm{TM}}$ devices without external logic. A serial strobe output allows direct connection to TMS320 family digital signal processors. The MAX186/MAX188 use either the internal clock or an external serial-interface clock to perform successive-approximation AOD conversions. The serial interface can operate beyond $4 \mathrm{MHz}$ when the internal clock is used.

The MAX186 has an internal 4.096V reference while the MAX 188 requires an external reference. Both parts have a reference-buffer amplifier that simplifies gain trim .

The MAX186/MAX188 provide a hard-wired SHDN pin and two-software-selectable power-down modes. Accessing the serial interface automatically powers up the devices, and the quick turn-on time allows the MAX186/MAX188 to be shut down between every conversion. Using this technique of powering down between conversions, supply current can be cut to under $10 \mu \mathrm{A}$ at reduced sampling rates.

The MAX186/MAX188 are avallable in 20-pin DIP and SO packages, and in a shrink small-oulline package (SSOP), that occupies $30 \%$ less area than an 8-pin DIP. For applications that call for a parallet interface, see the MAX180/MAX181 data sheet. For anti-aliasing filters,

consult the MAX274/MAX275 data sheet

Portable Data Logging

Data-Acquisition

High-Accuracy Process Control

Automatic Testing

Robotics

Battery-Powered Instruments

Medical instruments

IM SPI and QSPI are registered trademarks of Motorola.

Microwire is a registered trademark of Nalional Semiconductor.

11 $1 \times 19$

\section{MAXIM \\ Low-Power, 8-Channel, Serial 12-Bit ADCs}

- 8-Channel Single-Ended or 4-Channel

Differential Inputs

- Single $+5 \mathrm{~V}$ or $\pm 5 \mathrm{~V}$ Operation

- Low Power: 1.5mA (operating mode) $2 \mu \mathrm{A}$ (power-down mode)

- Internal Track/Hold, 133kHz Sampling Rate

- Internal 4.096V Reference (MAX186)

- SPI-, QSPI-, Microwire-, TMS320-Compatible 4-Wire Serial Interiace

- Software-Configurable Unipolar or Blpolar Inputs

- 20-Pin DIP, SO, SSOP Packages

- Evaluation Kit Available

Ordering Information

\begin{tabular}{|lll|}
\hline PART & TEMP. HANGE & PIN-PACKAGE \\
\hline MAX186_CPP & $0^{\circ} \mathrm{C} 10+70^{\circ} \mathrm{C}$ & 20 Plastic DIP \\
\hline MAX186_CWP & $0^{\circ} \mathrm{C} 10+70^{\circ} \mathrm{C}$ & 20 SO \\
\hline MAX186_CAP & $0^{\circ} \mathrm{C} 10+70^{\circ} \mathrm{C}$ & 20 SSOP \\
\hline MAX186DC/D & $0^{\circ} \mathrm{C} 10+70^{\circ} \mathrm{C}$ & Dice \\
\hline MAX186_EPP & $-40^{\circ} \mathrm{C}$ to $+85^{\circ} \mathrm{C}$ & 20 Plastic DIP \\
\hline MAX186_EWP & $-40^{\circ} \mathrm{C}$ to $+85^{\circ} \mathrm{C}$ & 20 SO \\
\hline MAX186_EAP & $-40^{\circ} \mathrm{C} 10+85^{\circ} \mathrm{C}$ & 20 SSOP \\
\hline MAX186_MUP & $-55^{\circ} \mathrm{C}$ to $+125^{\circ} \mathrm{C}$ & 20 CERDIP** \\
\hline
\end{tabular}

Ordering Informatlon continued on last page.

† NOTE: Parts are offered in grades A, B, C and D (grades defined in Electrical Characteristics). When ordering, please specity grade. Confact factory for availability of A-grade in SSOP package.

Confact factory for avaliability of A-grade in SSOP package.

- Contact factory for availability and processing to MIL-STD-883.

Pin Configuration

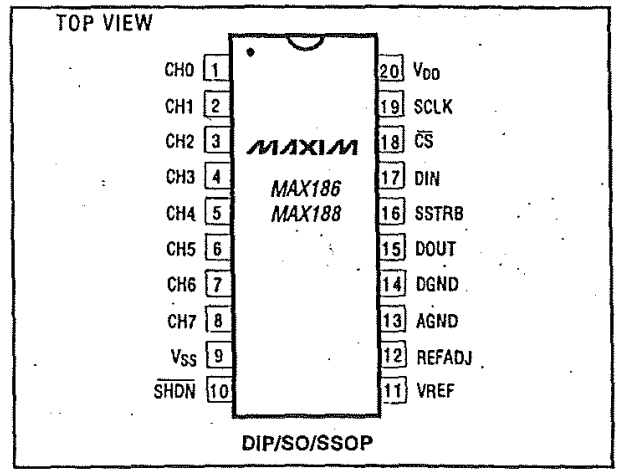

Maxim Integrated Products

For free samples \& the latest literature: http://www.maxim-ic.com, or phone 1-800-998-8800 


\section{Low-Power, 8-Channel, Serial 12-Bit ADCs}

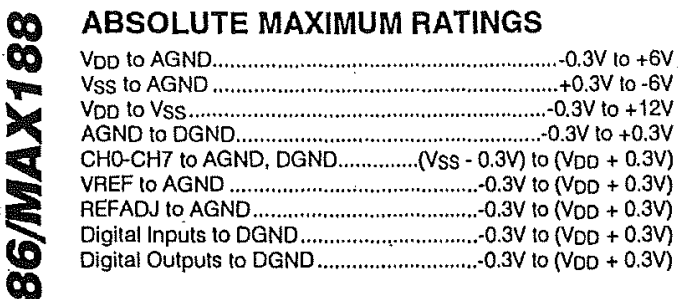

Continuous Power Dissipation $\left(T_{A}=+70^{\circ} \mathrm{C}\right)$

Plastic DIP (derate $11.11 \mathrm{~mW} /{ }^{\circ} \mathrm{C}$ above $+70^{\circ} \mathrm{C}$ ) $\ldots . . .889 \mathrm{~mW}$ SO (derate $10.00 \mathrm{~mW} /{ }^{\circ} \mathrm{C}$ above $+70^{\circ} \mathrm{C}$ ) ……...............800 $80 \mathrm{~mW}$ SSOP (deralo $800 \mathrm{~mW} /{ }^{\circ} \mathrm{C}$ abov $+70^{\circ} \mathrm{C}$ ) ..............640mW CERDip (derate $11.11 \mathrm{~mW} C$ abovo $+70 \mathrm{C}) \ldots \ldots \ldots \ldots . . .1889 \mathrm{~mW}$ Operating Temperature Ranges:

MAX186_ClMAX188_C....

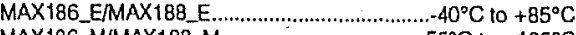

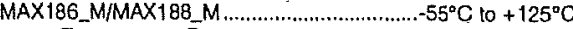
Storage Temperature Range ............................ $-60^{\circ} \mathrm{C}$ to $+150^{\circ} \mathrm{C}$ Lead Temperature (soldering, 10sec) .......................... $+300^{\circ} \mathrm{C}$

Stresses beyond those listed under "Absolute Maximum Ratings" may cause permanent demage to the device. These are stress ralings only, and funclional operation of the device at these or any other conditions beyond thase indicaled in the operational sections of the specifications is nof implied. Exposure to opsolute meximum rating conditions for exterded periods may alfect device reliability.

\section{TERISTICS}

$\left(V_{D D}=5 \mathrm{~V} \pm 5 \% ; V_{S S}=0 \mathrm{~V}\right.$ or $-5 \mathrm{~V} ; I_{\mathrm{CUK}}=2.0 \mathrm{MHz}$, external clock ( $50 \%$ duty cycle); 15 clocks/conversion cycle (133ksps); MAX $186-$ $4.7 \mu \mathrm{F}$ capacitor at VREF pin; MAX188 - external relerence, VREF $=4.096 \mathrm{~V}$ applied lo VREF pin; $T_{A}=T_{\text {MIN }}$ to $T_{\text {MAX }}$, unless otherwise noted.)

\begin{tabular}{|c|c|c|c|c|c|c|c|}
\hline PARAMETER & SYMBOL & \multicolumn{2}{|c|}{ CONDITIONS } & MIN & TYP & MAX & UNITS \\
\hline \multicolumn{8}{|l|}{ DC ACCURACY (Note 1) } \\
\hline Resolution & & & & 12 & & & Bits \\
\hline \multirow{5}{*}{ Relative Accuracy (Note 2) } & \multirow{5}{*}{. } & \multicolumn{2}{|l|}{ MAX186A/MAX18BA } & & & \pm 0.5 & \multirow{5}{*}{ LSB } \\
\hline & & \multicolumn{2}{|l|}{ MAX186B/MAX188B } & & & \pm 0.5 & \\
\hline & & \multicolumn{2}{|l|}{ MAX186C } & & & \pm 1.0 & \\
\hline & & \multicolumn{2}{|l|}{ MAX188C } & & & \pm 0.75 & \\
\hline & & \multicolumn{2}{|l|}{ MAX186D/MAX188D } & & & \pm 1.0 & \\
\hline Differential Nonlinearity & DNL & \multicolumn{2}{|c|}{ No missing codes over temperature } & & & \pm 1 & LSB \\
\hline \multirow{4}{*}{ Offset Error } & & \multicolumn{2}{|l|}{ MAX186AMAX188A } & & & \pm 2.0 & \multirow{4}{*}{ LSB } \\
\hline & & \multicolumn{2}{|l|}{ MAX186B/MAX188B } & & & \pm 3.0 & \\
\hline & & \multicolumn{2}{|l|}{ MAX186C/MAX188C } & & & \pm 3.0 & \\
\hline & & \multicolumn{2}{|l|}{ MAX186D/MAX188D } & & & \pm 3.0 & \\
\hline \multirow{5}{*}{ Gain Error (Note 3) } & & \multicolumn{2}{|l|}{ MAX186 (all grades) } & & & \pm 3.0 & \multirow{5}{*}{ LSB } \\
\hline & & \multirow{4}{*}{$\begin{array}{l}\text { External reference } \\
4.096 \mathrm{~V} \text { (MAX188) }\end{array}$} & MAX $188 A$ & & & \pm 1.5 & \\
\hline & & & MAX188B & & & \pm 2.0 & \\
\hline & & & MAX188C & & & \pm 2.0 & \\
\hline & & & MAX1B8D & & & \pm 3.0 & \\
\hline Gain Temperature Coefflcient & & \multicolumn{2}{|c|}{ External reference, $4.096 \mathrm{~V}$} & \multicolumn{3}{|c|}{ \pm 0.8} & $\mathrm{ppm} /{ }^{\circ} \mathrm{C}$ \\
\hline $\begin{array}{l}\text { Channel-to-Channel } \\
\text { Offset Matching }\end{array}$ & & & & \multicolumn{3}{|c|}{ \pm 0.1} & LSB \\
\hline \multicolumn{8}{|c|}{ DYNAMIC SPECIFICATIONS (10kHz sine wave input, $4.098 \mathrm{~V}$.P. $133 \mathrm{ksps}, 2.0 \mathrm{MHz}$ external clock, bipolar input mode) } \\
\hline Signal-to-Noise + Distortion Ratio & SINAD & & & 70 & & & $\mathrm{~dB}$ \\
\hline $\begin{array}{l}\text { Total Harmonic Distortion } \\
\text { (up to the } 5 \text { th harmonic) }\end{array}$ & THD & . & & & & -80 & dB \\
\hline Spurious-Free Dynamic Range & SFDR & & & 80 & & & $\mathrm{~dB}$ \\
\hline Channel-to-Channel Crosstalk & & $65 \mathrm{kHz}, V_{1 N}=4.096$ & $p($ Note 4$)$ & & -85 & & $\mathrm{~dB}$ \\
\hline
\end{tabular}




\section{Low-Power, 8-Channel, Serial 12-Bit ADCs}

\section{ELECTRICAL CHARACTERISTICS (continued)}

$\left(V_{D O}=5 \mathrm{~V} \pm 5 \% ; V_{S S}=O V\right.$ or $-5 V_{i} f_{C L K}=2.0 \mathrm{MHz}$, external clock ( $50 \%$ duty cycle); 15 clocks/conversion cycle (133ksps); MAX186 4.7 $\mu \mathrm{F}$ capacitor at VREF pin; MAX188 - external reference, VREF $=4.096 \mathrm{~V}$ applied to VREF pin; $T_{A}=T_{\text {MIN }}$ to $T_{\text {MAX }}$, unless otherwise noted.)

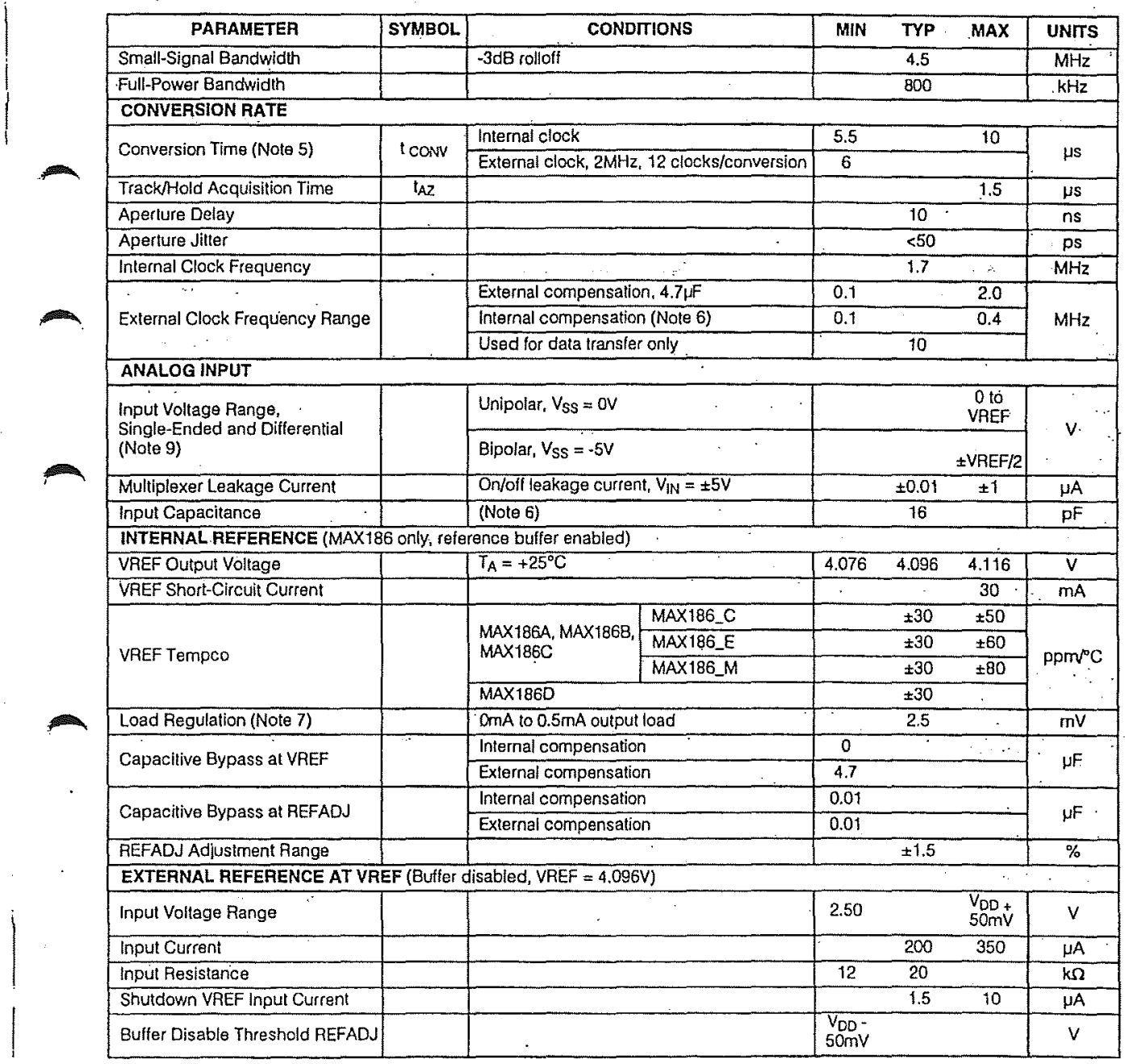




\section{Low-Power, 8-Channel, Serial 12.Bit ADCs}

9. ELECTRICAL CHARACTERISTICS (continued)

C. $\left(V_{D O}=5 \mathrm{~V} \pm 5 \% ; V_{S S}=O V\right.$ or $-5 V ; f C L K=2.0 \mathrm{MHz}$, external clock $(50 \%$ duty cycle); 15 clocks/conversion cycle (133ksps); MAX186 4.7jF capacitor at VREF pin; MAX188 - external reference, VREF $=4.096 \mathrm{~V}$ applied to VREF pin; $T_{A}=T_{\text {MIN }}$ to $T_{\text {MAX. }}$. unless otherwise noted.)

\begin{tabular}{|c|c|c|c|c|c|c|}
\hline PARAMETER & SYMBOL & CONDITIONS & MIN & TYP & MAX & UNITS \\
\hline \multicolumn{7}{|c|}{ EXTERNAL REFERENCE AT REFADJ } \\
\hline \multirow{2}{*}{ Capacilivo Bypass at VREF } & & Internal compensation mode & 0 & & & \multirow{2}{*}{$\mu F$} \\
\hline & & External compensation mode & 4.7 & & & \\
\hline \multirow{2}{*}{ Relerence-Buffer Gain } & & MAX186 & & 1.678 & & \multirow{2}{*}{ VN } \\
\hline & & MAX188 & & 1.638 & & \\
\hline \multirow{2}{*}{ REFADJ Input Current } & & MAX186 & & & \pm 50 & \multirow{2}{*}{$\mu \mathrm{A}$} \\
\hline & & MAX18B & & & \pm 5 & \\
\hline
\end{tabular}

DIGITAL INPUTS (DIN, SCLK, CS, SHDN)

DIN, SCLK, CS Inoul High Voltage

DIN, SCLK, CS Input Low Voltage $V_{\text {INL }}$

\begin{tabular}{|l|l|}
\hline DIN, SCLK, CS Input Hysteresis & $V_{\text {HYST }}$ \\
\hline DIN, SCLK, CS Input Leakege
\end{tabular}

DIN, SCLK, CS Input Leakage

DIN, SCLK, CS Input Capacitance $\mathrm{C}_{\mathbb{N}}$

SHDN Input High Voltage

SHDN Input Low Voltage

SHDN Input Current, High

SHDN input Currant, Low

SHDN Input Mid Voltage

SHDN Voltage, Floating

SHDN Max Allowed Leakage,

SHDN Max Al
Mid Input:

DIGITAL OUTPUTS (DOUT, SSTRB)

\begin{tabular}{|c|c|c|c|c|c|}
\hline \multirow{2}{*}{ Output Vollage Low } & \multirow{2}{*}{$V_{O L}$} & $I_{S I N K}=5 \mathrm{~mA}$ & & 0.4 & \multirow{2}{*}{ V } \\
\hline & & $I_{\text {SINK }}=16 \mathrm{~mA}$ & \multicolumn{2}{|l|}{0.3} & \\
\hline Output Voltage High & $\mathrm{V}_{\mathrm{OH}}$ & ISOURCE $=\operatorname{tmA}$ & 4 & & $\bar{V}$ \\
\hline Three-Stale Leakage Current & $T_{L}$ & $\overline{\mathrm{CS}}=5 \mathrm{~V}$ & & \pm 10 & $\mu \mathrm{A}$ \\
\hline Three-State Output Capacitance & Cout & $\overline{\mathrm{CS}}=5 \mathrm{~V}($ Nole 6$)$ & & 15 & $\mathrm{pF}$ \\
\hline \multicolumn{6}{|l|}{ POWER REQUIREMENTS } \\
\hline Positive Supply Voltage. & $V_{D D}$ & & $5 \pm 5 \%$ & & $\mathrm{~V}$ \\
\hline Negative Supply Voltage & $v_{S S}$ & & $\begin{array}{c}0 \text { or } \\
-5 \pm 5 \%\end{array}$ & & v \\
\hline \multirow{3}{*}{ Posittve Supply Current } & \multirow{3}{*}{ loo } & Operating mode & 1.5 & 2.5 & $\mathrm{~mA}$ \\
\hline & & Fast power-down & 30 & 70 & \multirow{2}{*}{$\mu \mathrm{A}$} \\
\hline & & Full power-down & 2 & 10 & \\
\hline \multirow{2}{*}{ Negative Supply Current } & \multirow{2}{*}{ Iss } & Operating mode and fast power-down & & 50 & \multirow{2}{*}{$\mu \mathrm{A}$} \\
\hline & & Full power-down & & 10 & \\
\hline
\end{tabular}




\section{Low-Power, 8-Channel, Serial $12 \mathrm{BBit}$ ADCs}

ELECTRICAL CHARACTERISTICS (continued)

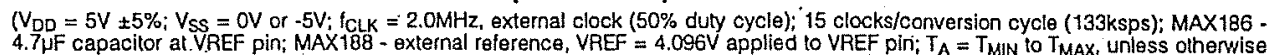
noted.)

\begin{tabular}{|c|c|c|c|c|c|c|}
\hline PARAMETEA & SYMBOL & CONDITIONS & MIN & TYP & MAX & UNTS \\
\hline $\begin{array}{l}\text { Positive Supply Rejection } \\
\text { (Note 8) }\end{array}$ & PSR & $\begin{array}{l}V_{D O}=5 \mathrm{~V} \pm 5 \% ; \text { external raference, } 4.096 \mathrm{~V} ; \\
\text { full-scale input }\end{array}$ & & \pm 0.06 & $\star 0.5$ & $\mathrm{mV}$ \\
\hline $\begin{array}{l}\text { Negative Supply Rejection } \\
\text { (Note 8) }\end{array}$ & PSR & 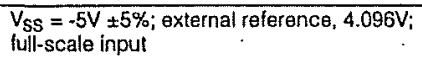 & & \pm 0.01 & \pm 0.5 & $\mathrm{mV}$ \\
\hline
\end{tabular}

(Note 8)

Note 1: Tested at $\mathrm{V}_{\mathrm{DO}}=5.0 \mathrm{~V} ; \mathrm{V}_{\mathrm{SS}}=0 \mathrm{~V}$; unipolar input mode.

Note 2: Relative accuracy is the deviation of the analog value at any code from its theoretical value after the full-scale range has been calibrated.

Note 3: MAX186 - internal reference, offset nulled; MAX188 - external reference (VREF $=\$ 4.096 \mathrm{~V}$ ), of set nulled.

Note 4: Ground on-channel; sine wave applied to all off channels.

Note 5: Conversion time defined as the number of clock cycles times the clock period; clock has $50 \%$ duty cycle.

Note 6: Guaranteed by design. Not subject to production lesting.

Note 7: External load should not change during conversion for specified accuracy.

Note 8: Measured at $V_{\text {SUPPLY }}+5 \%$ and $V_{\text {SUPPLY }}-5 \%$ only.

Note 9: The common-mode range for the analog inputs is from $V_{S S}$ to $V_{D D}$

\section{TIMING CHARACTERISTICS}

$\left(V_{D D}=5 V \pm 5 \% ; V_{S S}=O V\right.$ or $-5 V, T_{A}=T_{M I N}$ to $T_{M A X}$, unless othenwise noted.)

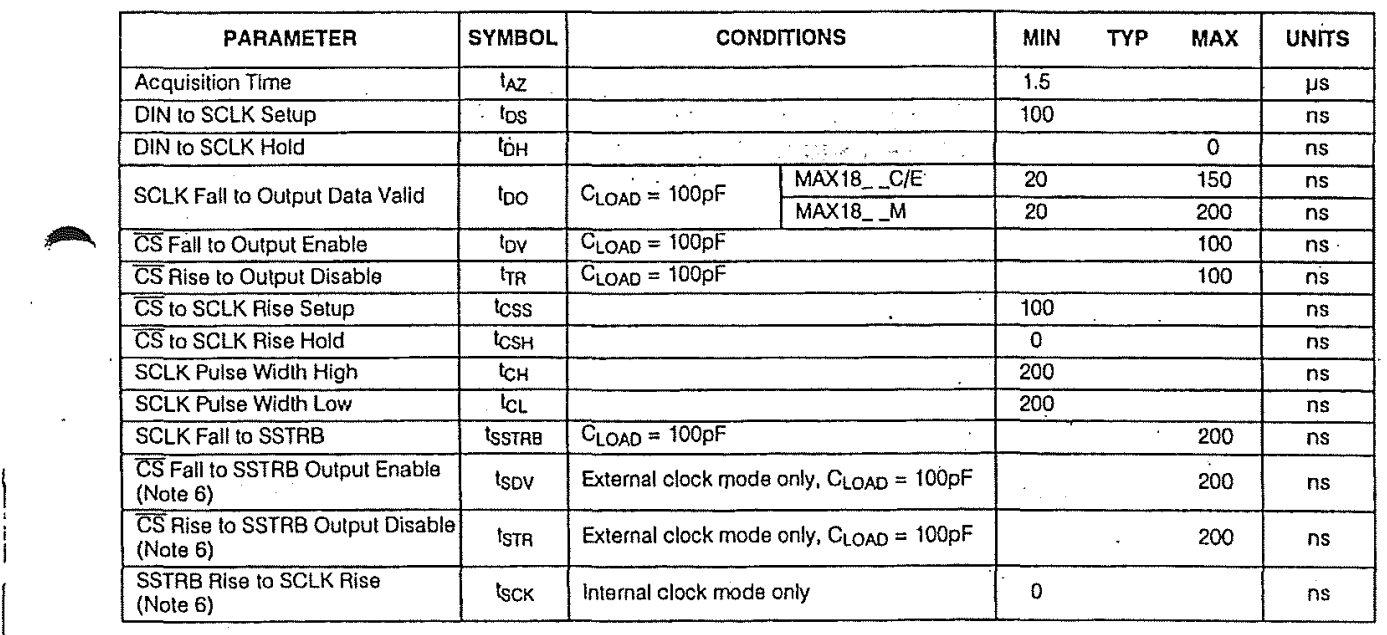




\section{Low-Power, 8-Channel, Serial 12-Bit ADCs}

Typical Operating Characteristics
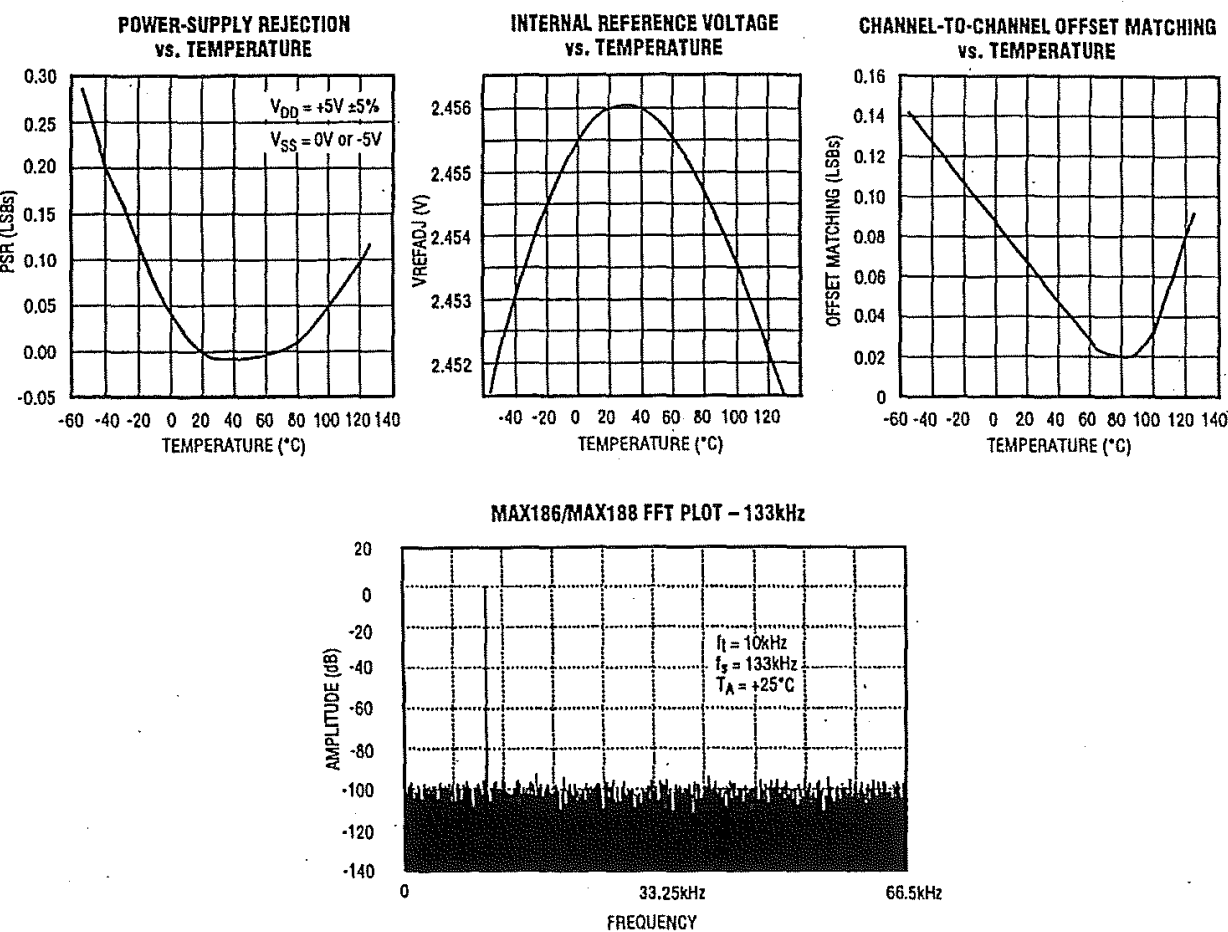

Pin Description

\begin{tabular}{|c|c|c|}
\hline PIN & NAME & FUNCTION \\
\hline 1.8 & $\mathrm{CHO}-\mathrm{CH}$.7 & Sampling Analog Inputs \\
\hline .9 & $v_{S S}$ & Negative Supply Voltage. Tie to $-5 \mathrm{~V} \pm 5 \%$ or AGND \\
\hline 10 & SHDN & $\begin{array}{l}\text { Three-Level Shutdown Input. Pulling SHDN low shuts the MAX186/MAX } 188 \text { down to } 10 \mu A \text { (max) } \\
\text { supply current, othenwise the MAX 185MMAX 188 are fully operational. Pulling SHDN high puts the } \\
\text { relerence-buffer amplifier in internal compensation mode. Letting SHDN float puts the } \\
\text { reference-buffer amplifier in external compensation mode. }\end{array}$ \\
\hline 11 & VREF & $\begin{array}{l}\text { Reference Voltage for analog.10-digital conversion. Also, Output of the Reference Buffer Amplifier } \\
\text { (4.096V in the MAX186, } 1.638 \times \times \text { REFADJ in the MAX183). Add a } 4.71 \mathrm{~F} \text { capacitor to ground when } \\
\text { using external compensation mode. Also functions as an input when used with a precision external } \\
\text { reference. }\end{array}$ \\
\hline
\end{tabular}




\section{Low-Power, 8-Channel, Serial 12-Bit ADCs}

Pin Description (continued)

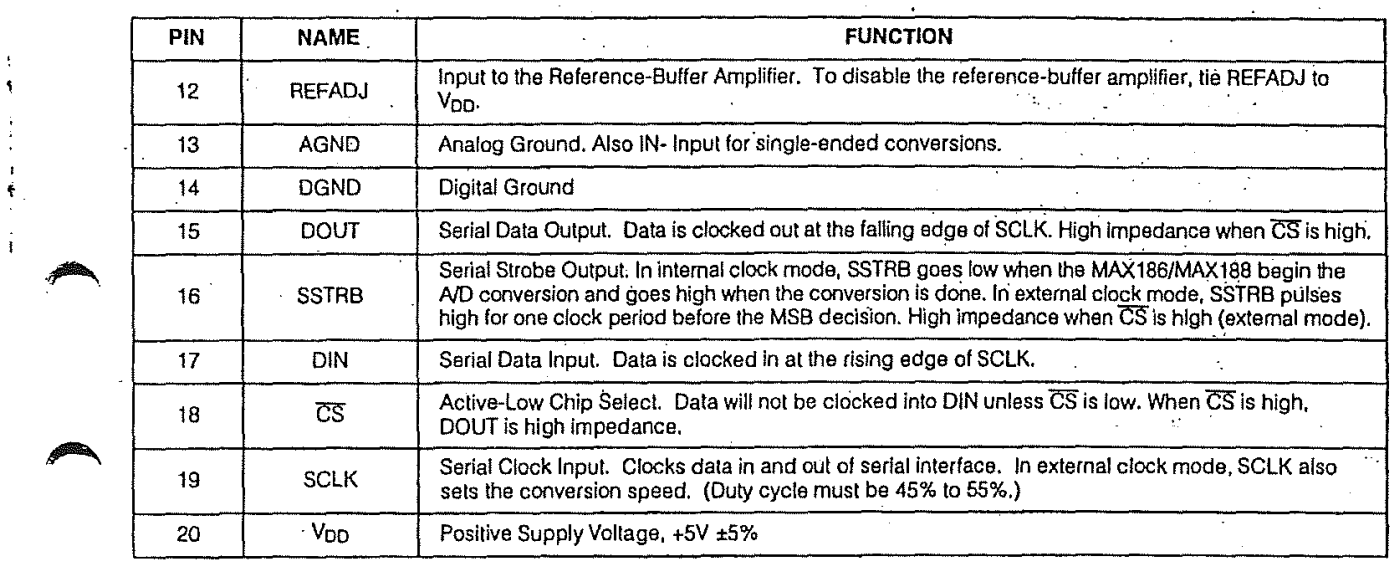

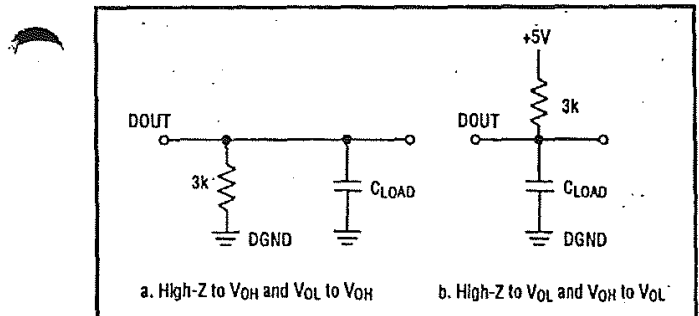

- Figure 1. Load Circuits for Enable Time

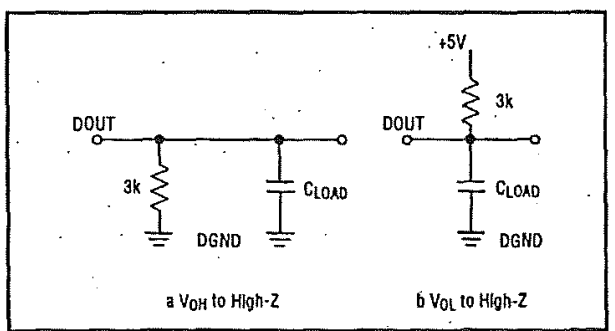

Figure 2. Load Circuits for Disabled Time

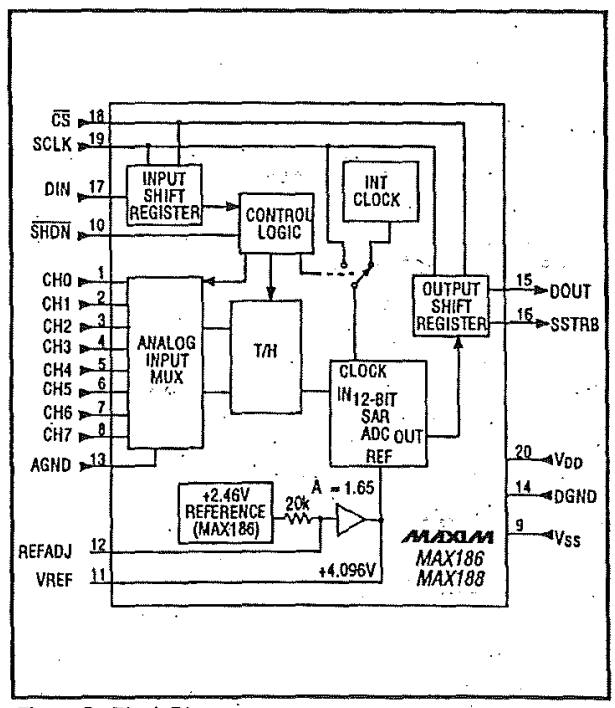

Figure 3. Block Diagram. 


\section{Low:Power, 8-Channel, Serial 12-Bit ADCs}

Detailled Description

The MAX186/MAX188 use a successive-approximation conversion technique and input track/hold $(T / H)$ circuity to convert an analog signal to a 12-bit digital output. A flexible serlal interface provides easy interface to microprocessors. No exlernal hold capacitors are required. Figure 3 shows the block dlagram for the MAX186/MAX188.

Pseudo-Differential Input

The sampling architecture of the ADC's analog comparator is illustrated in the Equivalent Input Circuit (Figure 4). In single-ended mode, $\mathrm{N}+$ is Internally switched to $\mathrm{CHO}-\mathrm{CH} 7$ and $\mathbb{I N}$ - is switched to AGND. In differential mode. $\mathbb{N}+$ and $\mathbb{N}$ - are selected from pairs of $\mathrm{CHO} / \mathrm{CH} 1, \mathrm{CH} 2 / \mathrm{CH} 3, \mathrm{CH} 4 / \mathrm{CH} 5$ and $\mathrm{CH} 6 / \mathrm{CH} 7$. Configure the channels with Table 3 and Table 4.

in differential mode, $\mathbb{N}$ - and $\mathbb{I N}+$ are internally switched to either one of the analog inputs. This configuration is pseudo-differential to the effect that only the signal at $\mathbb{I N +}$ is sampled. The return side (IN-) must remain stable within $\pm 0.5 \mathrm{LSB}( \pm 0.1 \mathrm{LSB}$ for best results) with respect to AGND during a conversion. Accomplish this by connecting a $0.1 \mu \mathrm{F}$ capacitor from AIN- (the selected analog input, respectively) to AGND.

During the acquisition interval, the channel selected as the positive input $\left(\mathrm{N}_{+}\right)$charges capacitor $\mathrm{C}_{\mathrm{HOLO}}$. The acquisition interval spans three SCLK cycles and ends on the falling SCLK edge after the last bit of the input control word has been entered. At the end of the acquisittion interval, the $T / H$ switch opens, retalning charge on $\mathrm{C}_{\text {HOLD }}$ as a sample of the signal at $\mathrm{IN+}$.

The conversion interval begins with the input multiplexer switching $\mathrm{C}_{H O L D}$ from the positive input $(\mathbb{N}+)$ to the negative input ( $\mathbb{N}-$-). In single-ended mode, $\mathbb{N}$ - is simply AGND. This unbalances node ZERO at the input of the comparator. The capacitive DAC adjusts during the remainder of the conversion cycle to restore node ZERO to OV within the limits of 12-bit resolution. This action is equivalent to transferring a charge of $16 \mathrm{pF} \times$ $\left[\left(V_{\left.N_{N}+\right)}-\left(V_{1 N}-\right)\right]\right.$ from $C_{H O L D}$ to the binary-weighted capacitive $D A C$, which in turn forms a digital representation of the analog inpul signal.

Track/Hold

The T/H enters its tracking mode on the falling clock edge after the fitth bit of the 8-bit control word has been shifted in. The $T / H$ enters its hold mode on the falling clock edge after the eighth bit of the control word has

8

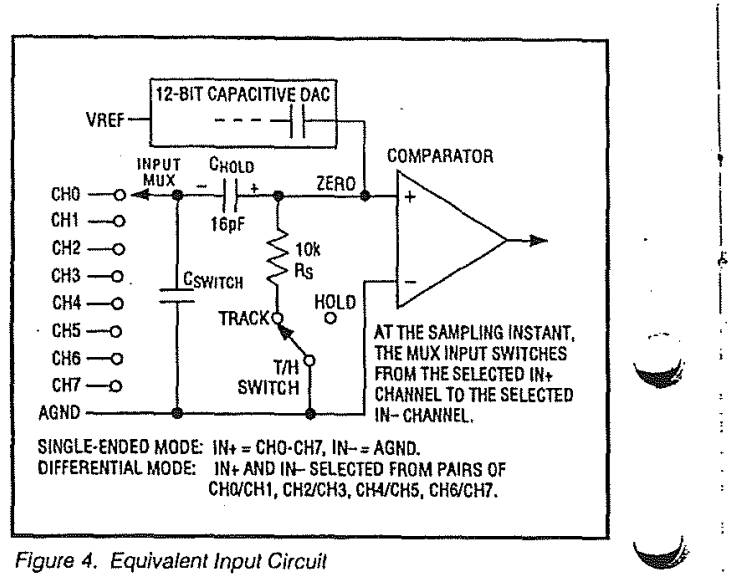

been shifted in. If the converter is sel up for single-ended inputs, IN- is connected to AGND, and the converter samples the " $t$ " input. If the converter is set up for differential inputs, IN-connects to the "-" input, and the difference of $|\mathbb{N}+-| N-\mid$ is sampled. At the end of the conversion, the positive input connects back to $\mathrm{IN}+$, and $\mathrm{C}_{\text {HOLO }}$ charges to the input signal.

The time required for the $T / H$ to acquire an input signal is a function of how quickly its input capacitance is charged. If the input signal's source impedance is high, the acqulsition time lengthens and more time must be allowed between conversions. Acquisition time is calculated by:

$$
t_{A Z}=9 \times\left(R_{S}+R_{I N}\right) \times 16 p F .
$$

where $R_{I N}=5 k \Omega, R_{s}=$ the source impedance of the input signal, and $t_{A Z}$ is never less than $1.5 \mu \mathrm{s}$. Note that source impedances below $5 \mathrm{k} \Omega$ do not significantly affect the $A C$ performance of the ADC.

Input Bandwidth

The ADC's. input tracking circuitry has a $4.5 \mathrm{MHz}$ small-signal bandwidth, so it is possible to digitize high-speed transient events and measure periodic signals with bandwidths exceeding the ADC's sampling rate by using undersampling techniques. To avoid high-frequency signals being aliased into the frequency band of interest, anti-alias filtering is recommended. See the MAX274/MAX275 continuous-time filters data sheet. 


\section{Low-Power, 8-Channel, Serial 12-Bit ADCs}

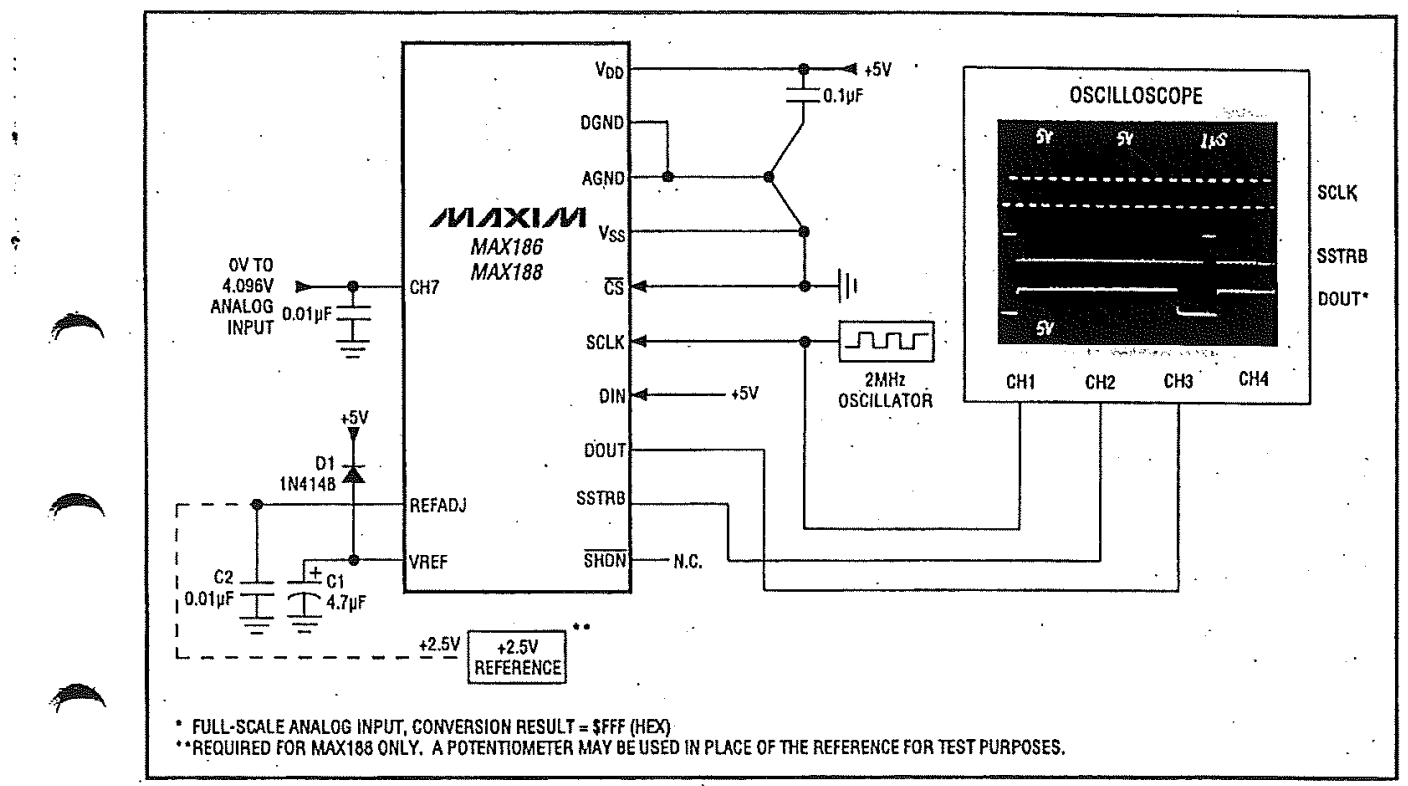

Figure 5. Quick-Look Circuit

Analog. Input Range and Input Protection Internal protection diodes, which clamp the analog inpul to $V_{D O}$ and $V_{S S}$, allow the channel input pins to swing from $V_{S S}-0.3 V$ to $V_{D D}+0.3 V$ without damage. However, for accurate conversions near full scale, the inputs must not exceed $V_{D D}$ by more than $50 \mathrm{mV}$, or be lower than $V_{S S}$ by $50 \mathrm{mV}$.

If the analog input exceeds $50 \mathrm{mV}$ beyond the supplies, do not forward bias the protection diodes of off-channels over two milliamperes, as excessive current will degrade the conversion accuracy of the on-channel.

The full-scale input voltage depends on the voltage at VREF, See Tables $1 a$ and $t b$.

Quick Look

To evaluate the analog performance of the MAX186/MAX188 quickly, use the circuit of Figure 5. The MAX186/MAX188 require a control byte to be written to DIN before each conversion. Tying DIN to $+5 \mathrm{~V}$ feeds in control bytes of \$FF (HEX). which trigger
Table ta. Unipolar Full Scale and Zero Scale

\begin{tabular}{|l|c|c|}
\hline \multicolumn{1}{|c|}{ Reference } & $\begin{array}{c}\text { Zero } \\
\text { Scale }\end{array}$ & Full Scale \\
\hline $\begin{array}{l}\text { Internal Reference } \\
\text { (MAX186 only) }\end{array}$ & OV & $+4.096 \mathrm{~V}$ \\
\hline $\begin{array}{l}\text { External Reference } \\
\text { at REFADJ }\end{array}$ & OV & $V_{\text {REFAD } \times A^{*}}$ \\
\hline at VREF & OV & VREF \\
\hline
\end{tabular}

- $A=1.678$ for the MAX186, 1.638 for the MAX188

Table to. Bipolar Full Scale, Zero Scale, and Negative Full Scale

\begin{tabular}{|c|c|c|c|}
\hline Reference & $\begin{array}{l}\text { Negative } \\
\text { Full Scale }\end{array}$ & $\begin{array}{l}\text { Zero } \\
\text { Scale }\end{array}$ & Full Scale \\
\hline $\begin{array}{l}\text { Inlernal Reference } \\
\text { (MAX186 only) }\end{array}$ & $-4.096 V / 2$ & OV & $+4.096 \mathrm{~V} / 2$ \\
\hline $\begin{array}{l}\text { External Reference } \\
\text { at REFADJ }\end{array}$ & $\begin{array}{c}-1 / 2 V_{\text {REFAD }} \\
\times A^{*}\end{array}$ & OV & $\begin{array}{c}+1 / 2 V_{\text {REFADJ }} \\
\times A^{*}\end{array}$ \\
\hline at VREF & $-1 / 2$ VREF & ov & $+1 / 2$ VREF \\
\hline
\end{tabular}

- $A=1.678$ for the MAX186, 1.638 for the MAX188 


\section{Low-Power, 8-Channely Serial 12-Bit ADCs}

single-ended unipolar conversions on $\mathrm{CH} 7$ in external clock mode without powering down between conversions. In external clock mode, the SSTRB output pulses high for one clock period before the most significant bit of the 12-bit conversion result comes out of DOUT. Varying the analog input to $\mathrm{CH} 7$ should alter the sequence of bits from DOUT. A total of 15 clock cycles is required per conversion. All transitions of the SSTRB and DOUT outputs occur on the falling edge of SCLK.

How to Start a Conversion A conversion is started on the MAX186/MAX188 by clocking a control byte into DIN: Each rising edge on SCLK, with CS low, clocks a bit from DIN into the MAX186/MAX188's internal shift reglster. After CS falls, the first arriving logic "1" bit defines the MSB of the control byte. Until this first "start" bit arrives, any numcontrol byte. "Until this first "start" bit arrives, any numeffect. Table 2 shows the control-byte format.

The MAX186/MAX188 are fully compatible with Microwire and SPI devices. For SPI, select the correct clock polarity and sampling edge in the SPI control registers: set $\mathrm{CPOL}=0$ and $\mathrm{CPHA}=0$. Microwire and $\mathrm{SPI}$ both transmit a byte and recelve a byte at the same time. Using the Typical Operating Circuit, the simplest software interlace requires only three 8-bit transfers to perform a conversion (one 8-bit transfer to configure the $A D C$, and two more 8-bit transfers to clock out the 12-bit conversion result).

Example: Simple Software Interface

Make sure the CPU's serial interface runs in master mode so the CPU generates the serial clock. Choose a clock frequency from $100 \mathrm{kHz}$ to $2 \mathrm{MHz}$.

1. Set up the control byte for external clock mode, call it T81. TB1 should be of the format: $1 \times X X X X 11$

Table 2. Control-Byte Format

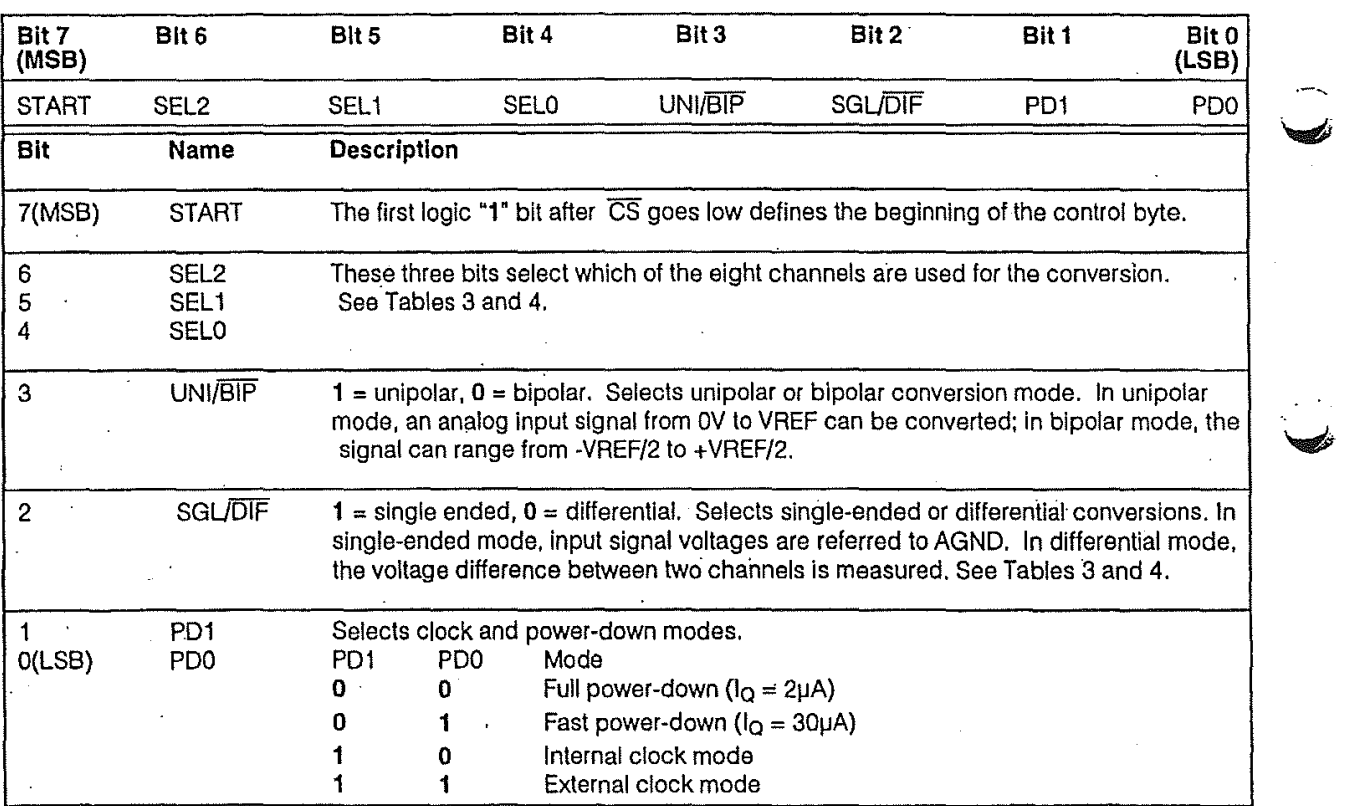




\section{Low-Power, 8-Channel, Serial 12-Bit ADCs}

Table 3. Channel Selection in Single-Ended Mode (SGLDTFF =1)

\begin{tabular}{|c|c|c|c|c|c|c|c|c|c|c|c|}
\hline SEL2 & SEL1 & SELO & CHO & $\mathrm{CH} 1$ & $\mathrm{CH} 2$ & $\mathrm{CH} 3$ & $\mathrm{CH} 4$ & CH5 & CH6 & $\mathrm{CH} 7$ & AGND \\
\hline 0 & 0 & 0 & + & & & & & & & & - \\
\hline 1 & 0 & 0 & & + & & & & 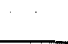 & & & - \\
\hline 0 & 0 & 1 & & & + & & & & & & - \\
\hline 1 & 0 & 1 & & & & + & & & & & - \\
\hline 0 & 1 & 0 & & & & & + & & & & - \\
\hline 1 & 1 & 0 & . & & & & & + & & & - \\
\hline 0 & 1 & 1 & & & & & . & & + & & - \\
\hline 1 & 1 & 1 & & & & & & & & + & - \\
\hline
\end{tabular}

Table 4. Channel Selection in Differential Mode (SGLDTFF $=0$ )

\begin{tabular}{|c|c|c|c|c|c|c|c|c|c|c|}
\hline SEL2 & SEL1 & SELO & $\mathrm{CHO}$ & $\mathrm{CH}_{1}$ & $\mathrm{CH} 2$ & $\mathrm{CH} 3$ & $\mathrm{CH} 4$ & $\mathrm{CH} 5$ & CH6 & $\mathrm{CH7}$ \\
\hline 0 & 0 & 0 & + & - & & & & & & \\
\hline 0 & 0 & 1 & & & + & - & & & & \\
\hline 0 & 1 & 0 & & & & & + & - & & \\
\hline 0 & 1 & 1 & & & & & & & + & - \\
\hline 1 & 0 & 0 & - & + & & & & & 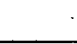 & . \\
\hline 1 & 0 & 1 & & & - & + & & $\ddots$ & & \\
\hline 1 & 1 & 0 & & & & & - & + & & \\
\hline 1 & 1 & 1 & & & & & & & - & + \\
\hline
\end{tabular}

Binary, where the $X s$ denote the particular channel and conversion-mode selected.

2. Use a general-purpose 1/O line on the CPU to pull CS on the MAX186/MAX188 low.

3. Transmit TB1 and simultaneously recelve a byle and call it RB1. Ignore RB1.

4. Transmit a byte of all zeros $(\$ 00 \mathrm{HEX})$ and simultaneously receive byte RB2.

5. Transmit a byte of alf zeros $(\$ 00 \mathrm{HEX})$ and simulaneously receive byte RB3.

6. Pull $\overline{\mathrm{CS}}$ on the MAX186/MAX188 high.
Figure 6 shows the timing for this sequence. Bytes RB2 and RB3 will contain the resutt of the conversion padded with one leading zero and three trailing zeros. The total conversion time is a function of the serial clock frequency and the amount of dead time between 8-bit transfers. Make sure that the total conversion time does not exceed $120 \mu \mathrm{s}$, to avoid excessive $\mathrm{T} / \mathrm{H}$ droop. Digital Output

In unipolar input mode, the output is straight binary (see Figure 15). For bipolar inputs, the output is twos-complement (see Figure 16). Data is clocked out at the falling edge of SCLK in MSB-first format. 


\section{Low-Power, 8-Channel, Serial 12-Bit ADCs}

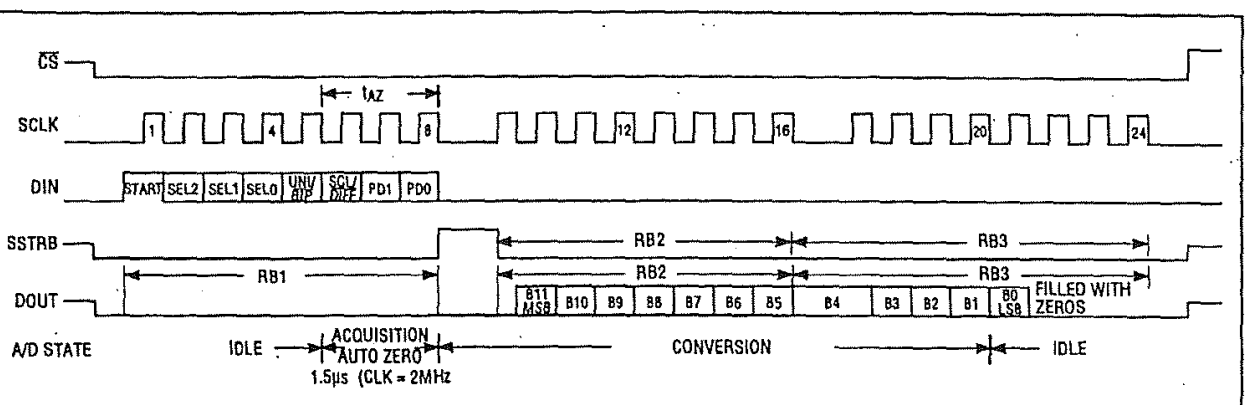

Figure 6. 24-Bit External Clock Mode Conversion Timing (SPI, QSPl and Microwire Compatible)

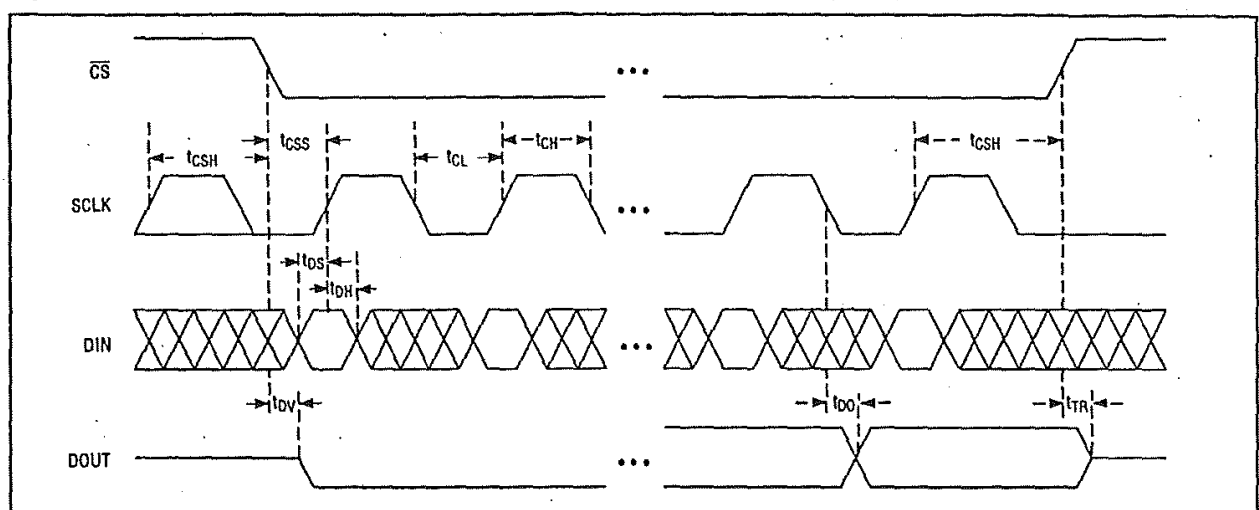

Figure 7. Detailed Serial-Interface Timing

Internal and Extermal Clock Modes

The MAX186/MAX188 may use either an external serial clock or the internal clock to perform the successive-approximation conversion. In both clock modes, the external clock shifts data in and out of the MAX186/MAX188. The T/H acquires the input signal as the last three bits of the control byte are clocked into DIN. Bits PD1 and PDO of the control byte program the clock mode. Figures 7 through 10 show the timing characteristics common to both modes.

External Clock

In external clock mode, the external clock not only shifts data in and out, it also drives the analog-to-digital con- version steps. SSTRB pulses high for one clock period after the last bit of the control byte Successive-approximation bit decisions are made and appear at DOUT on each of the next 12 SCLK falling edges (see Figure 6). SSTAB and DOUT go into a high-impedance state when $\overline{\mathrm{CS}}$ goes high; after the next $\overline{\mathrm{CS}}$ falling edge, SSTRB will output a logic low. Figure 8 shows the SSTRB timing in externai clock mode.

The conversion must complete in some minimum time, or else droop on the sample-and-hold capacitors may degrade conversion results. Use internal clock mode if the clock period exceeds $10 \mu \mathrm{s}$, or if serial-clock interruptions could cause the conversion interval to exceed $120 \mu \mathrm{s}$. 


\section{Low-Power, 8-Channel, Serial 12-Bit ADCs}

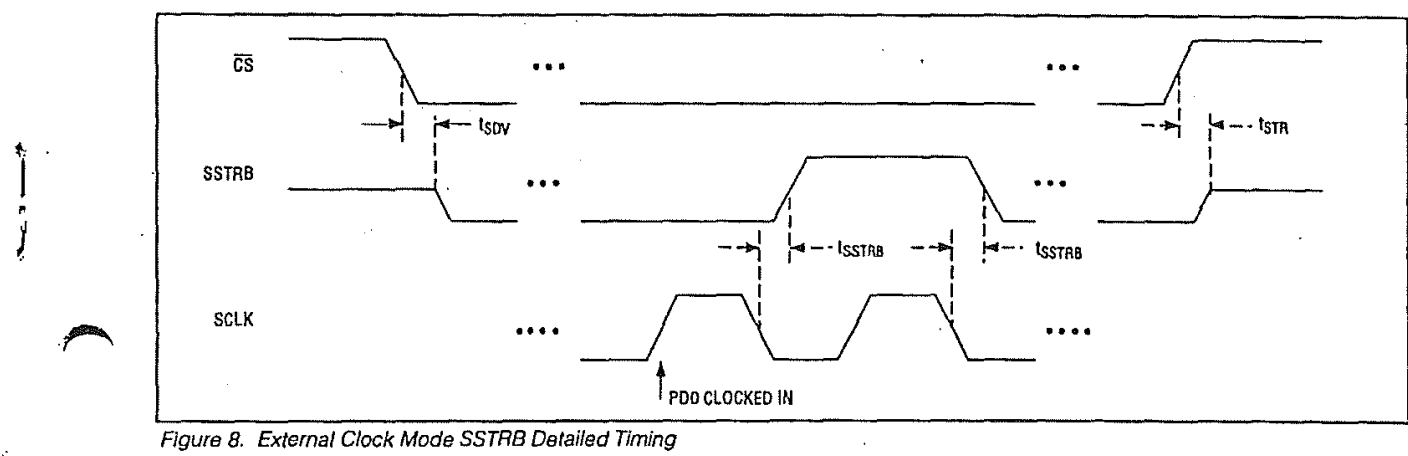

Figure 8. External Clock Mode SSTAB Detailed Timing

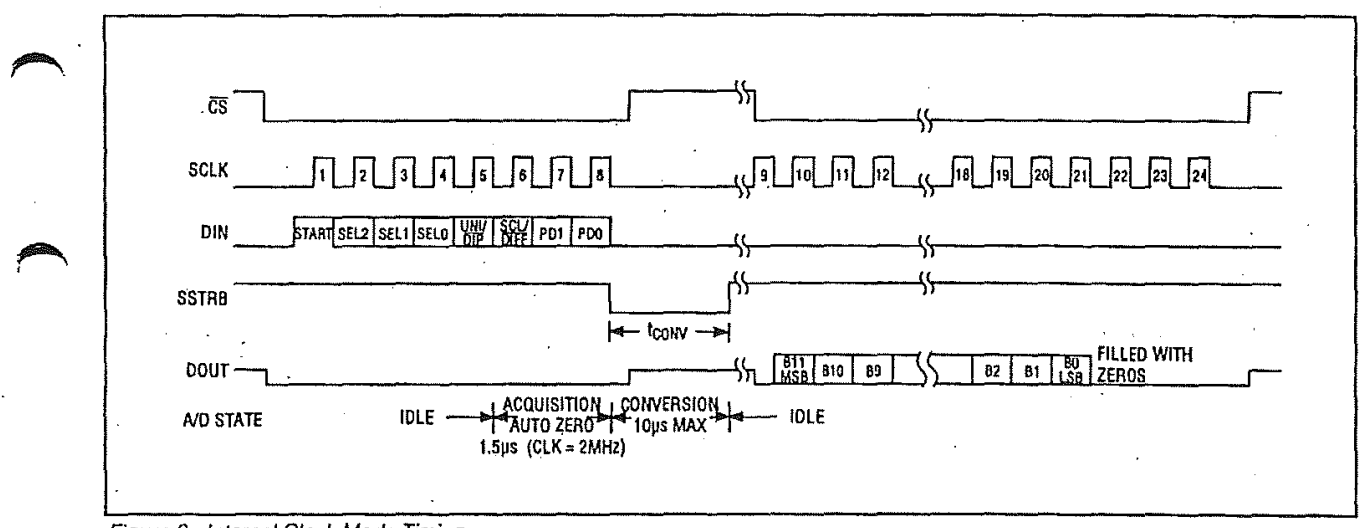

Figure 9. Internal Clock Mode Timing

Internal Clock

In internal clock mode, the MAX186/MAX188 generate their own conversion clock internally. This frees the microprocessor from the burden of running the SAR conversion clock, and allows the conversion results to be read back at the processor's convenience, at any clock rate from zero to typically $10 \mathrm{MHz}$. SSTRB goes low at the start of the conversion and then goes high when the conversion is complete. SSTRB will be low for a maximum of 10us, during which time SCLK should remain low for best noise performance. An internal register stores data when the conversion is in progress. SCLK clocks the data out at this register at any time after the conversion is complete. After SSTRB goes high, the next falling clock edge will produce the MSB of the conversion at DOUT, followad by the remaining bits in MSB-first format (see Figure 9). $\overline{C S}$ does not need to be held low once a conversion is started. Pulling $\overline{\mathrm{CS}}$ high prevents data from being clocked into the MAX186/MAX188 and three-states DOUT, but it does not adversely effect an intemal clock-mode conversion already in progress. When internal clock mode is selected, SSTRB does not go into a high-impedance state when $\overline{\mathrm{CS}}$ goes high.

Figure 10 shows the SSTRB timing in internal clock mode. In internal clock mode, data can be shifted in and out of the MAX186/MAX188 at clock rates exceeding $4.0 \mathrm{MHz}$, provided that the minimum acquisition time, $t_{\mathrm{AZ}}$. is kept above $1.5 \mu \mathrm{s}$. 


\section{Low-Power, 8-Channel, Serial 12-Bit ADCs}

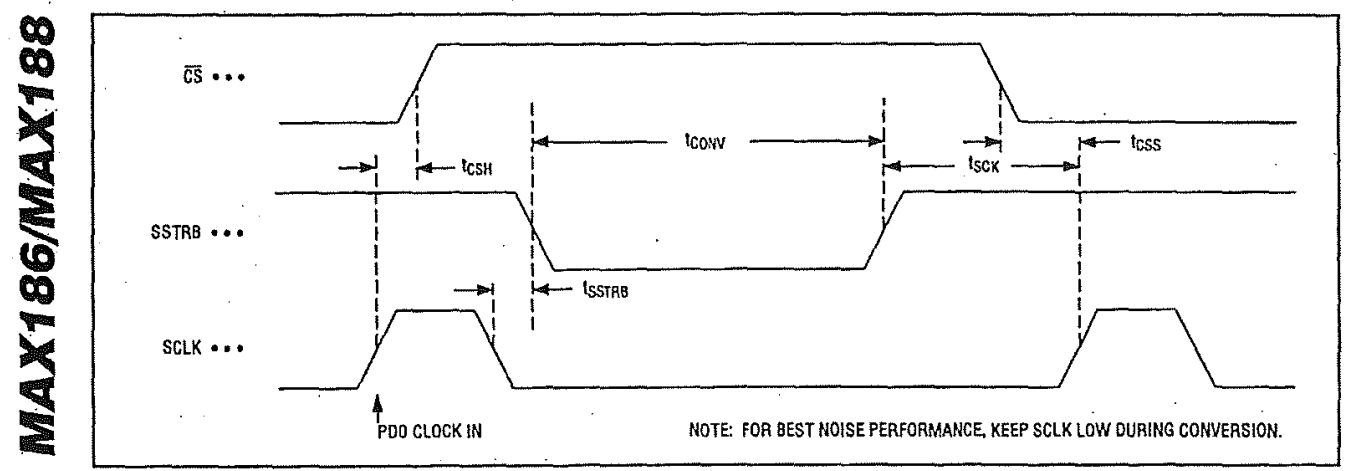

Figure 10. Internal Clock Mode SSTRB Detailed Timing

Data Framing

The falling edge of $\overline{\mathrm{CS}}$ does not start a conversion on the MAX186/MAX188. The first logic high clocked into DIN is interpreted as a start bit and defines the first bil of the control byte. A conversion starts on the falling edge of SCLK, after the eighth bit of the control byte (the PDO bit) is clocked into.DIN. The start bit is defined as:

The first high bit clocked into DIN with $\overline{\mathrm{CS}}$ low any-

time the converter is idle, e.g. after $V_{C C}$ is applied.

$$
\text { OR }
$$

The first high bit clocked into DIN after bit 5 of a conversion in progress is clocked onto the DOUT pin. If a falling edge on $\overline{\mathrm{CS}}$ forces a start bit before bit 5 (B5) becomes available, then the current conversion will be terminated and a new one started. Thus, the fastest the MAX186/MAX188 can run is 15 clocks per conversion. Figure 1 fa shows the serial-interface timing necessary to perform a conversion every 15 SCLK cycles in external clock mode.

Most microcontrollers require that conversions occur in multiples of 8 SCLK clocks; 16 clocks per conversion will typlcally be the fastest that a microcontroller can drive the MAX186/MAX188. Figure 11b shows the serial-interface timing necessary to perform a conversion every 16 SCLK cycles in external clock mode.

\section{Applications Information}

Power-On Reset

When power is first applied and if SHDN is not pulled low, internal power-on reset circuitry will activate the
MAX186/MAX188 in internal clock mode, ready to convert with SSTRB $=$ high. After the power supplies have been slabilized, the internal reset time is $100 \mu$ s and no conversions should be performed during this phase. SSTRB is high on power-up and, if $\overline{\mathrm{CS}}$ is low, the first logical 1 on DIN will be interpreted as a start bil. Unill a conversion takes place, DOUT will shift out zeros.

\section{Reference-Buffer Compensation}

In addition to its shutdown function, the SHDN pin also selects internal or external compensation. The compensation affects both power-up time and maximum conversion speed. Compensated or not, the minimum clock rate is $100 \mathrm{kHz}$ due to droop on the sample-and-hold.

To select external compensation, float SHDN. See the Typical Operating Circuit, which uses a $4.7 \mu \mathrm{F}$ capacitor at VREF. A value of $4.7 \mu \mathrm{F}$ or greater ensures stability and allows operation of the converter at the full clock speed of $2 \mathrm{MHz}$. External compensation increases power-up time (see the Choosing Power-Down Mode section, and Table 5). Internal compensation requires no external capacitor at VREF, and is selecled by pulling SHDN high. Internal compensation allows for shortest power-up times, but is only available using an external clock and reduces the maximum clock rate to $400 \mathrm{kHz}$.

Power-Down

Choosing Power-Down Mode

You can save power by placing the converter in a low-current shutdown state between conversions. Select full power-down or fast power-down mode via bits 7 and 8 of the DIN control byte with SHDN high 


\section{Low-Power, 8-Channel,} Serial 1 2Bit ADCs

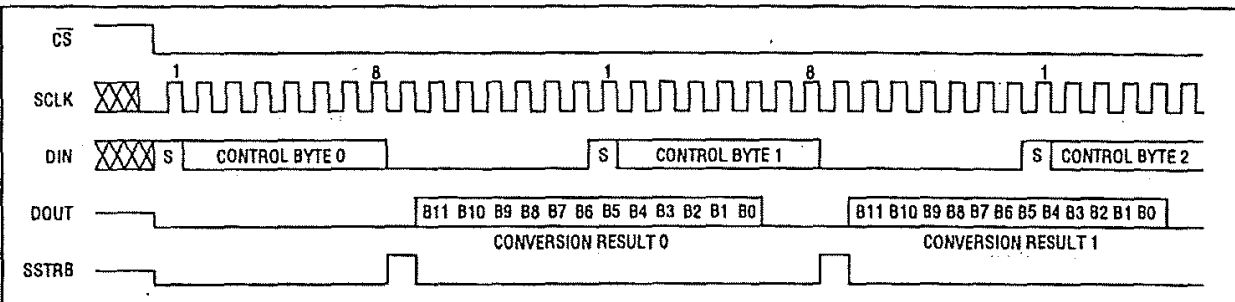

Figure 11a. External Clock Made, 15 Clocks/Conversion Timing

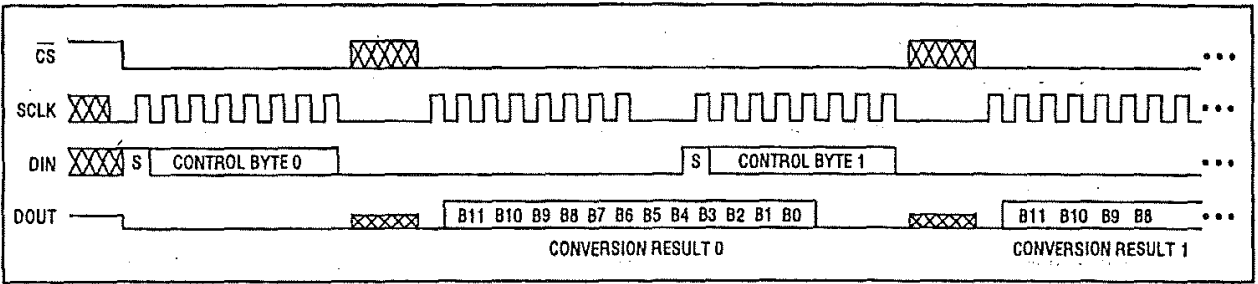

Figure 11b. External Clock Mode, 16 Clocks/Conversion Timing

(see Tables 2 and 6). Pull SHDN low at any time to shut down the converter completely. SHDN overrides bits 7 and 8 of DIN word (see Table 7).

Full power-down mode turns off all chip functions that draw quiescent current, reducing loD and iss typically to $2 \mu \mathrm{A}$.

Fast power-down mode turns off all circuitry except the bandgap reference. With the fast power-down mode, the supply current is $30 \mu \mathrm{A}$. Power-up time can be shortened to 5 us in internal compensation mode.

in both software shutdown modes, the serial interface remains operational, however, the ADC will not convert. Table 5 illustrates how the choice of reference-buffer compensation and power-down mode affects both power-up delay and maximum sample rate.

In external compensalion mode, the power-up time is $20 \mathrm{~ms}$ with a $4.7 \mu \mathrm{F}$ compensation capacitor $(200 \mathrm{~ms}$ with a $33 \mu \mathrm{F}$ capacitor) when the capacitor is fully discharged. in fast power-down, you can eliminate start-up time by using low-leakage capacitors that will not discharge more than 1/2LSB while shut down. In shutdown, the capacitor has to supply the current into the reference (1.5uA typ) and the transient currents at power-up.

Figures $12 \mathrm{a}$ and $12 \mathrm{~b}$ illustrate the various power-down sequences in both external and internal clock modes.

Software power-down is activated using bits PD1 and PDO of the control byte. As shown in Table 6, PD1 and PDO also specify the clock mode. When soltware shutdown is asserted, the ADC will continue to operate in the last specified clock mode until the conversion is complete. Then the ADC powers down into a low quiescent-current state. In internal clock mode, the interface remains active and conversion results may be clocked out while the MAX186/MAX188 have already entered a software power-down.

The first logical 1 on DIN will be interpreted as a start bit, and powers up the MAX186/MAX188. Following the start bit, the data input word or control byte also determines clock and power-down modes. For example, if the DIN word contains PDI $=1$, then the chip will remain powered up. If $\mathrm{PDY}=0$, a power-down will resume after one conversion.

Hardware Power-Down

The SHDN pin places the converter into the full power-down mode. Unlike with the software shut-down modes, conversion is not completed. It slops coincidentally with SHDN being brought low. There is no power-up delay if an external reference is used and is 


\section{Low-Power, 8-Channel, Serial 12-Bit ADCs}

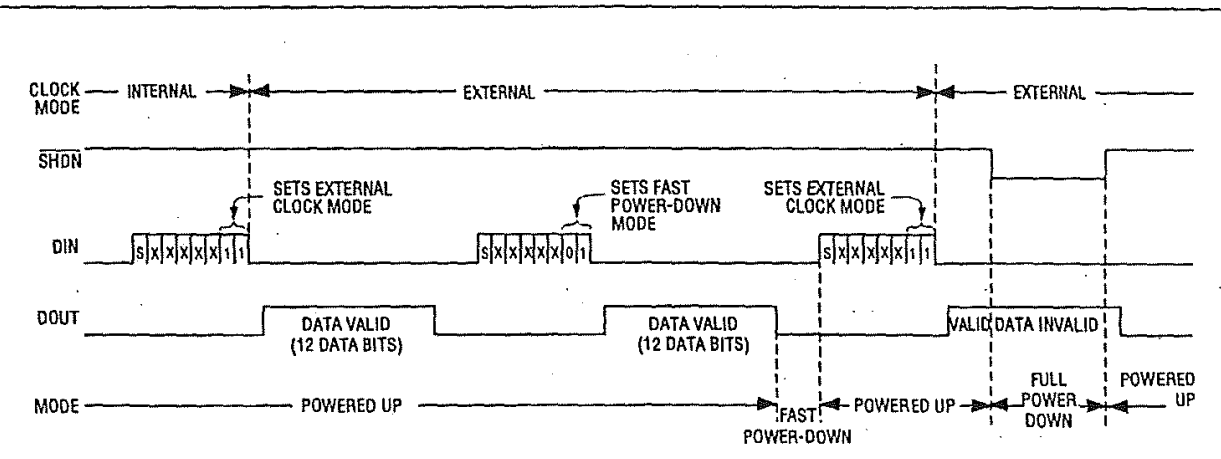

Figure 12a. Timing Diagram Power-Down Modes, External Clock

Table 5. Typical Power-Up Delay Times

\begin{tabular}{|llllll|}
\hline $\begin{array}{l}\text { Reference } \\
\text { Buffer }\end{array}$ & $\begin{array}{l}\text { Reference- } \\
\text { Buffer } \\
\text { Compensation } \\
\text { Mode }\end{array}$ & $\begin{array}{l}\text { VREF } \\
\text { Capacitor } \\
(\mu \mathrm{F})\end{array}$ & $\begin{array}{l}\text { Power- } \\
\text { Down } \\
\text { Mode }\end{array}$ & $\begin{array}{l}\text { Power-Up } \\
\text { Delay } \\
\text { (sec) }\end{array}$ & $\begin{array}{c}\text { Maximum } \\
\text { Sampling } \\
\text { Rate (ksps) }\end{array}$ \\
\hline Enabled & Internal & Fast & $5 \mu$ & 26 \\
\hline Enabled & Internal & & Full & 300 & 26 \\
\hline Enabled & External & 4.7 & Fast & See Figure 14c & 133 \\
\hline Enabled & External & 4.7 & Full & See Figure 14c & 133 \\
\hline Disabled & & Fast & $2 \mu$ & 133 \\
\hline Disabled & & Full & $2 \mu$ & 133 \\
\hline
\end{tabular}

Table 6. Soltware Shutdown and Clock Mode

\begin{tabular}{|ccl|}
\hline PD1 & PDo & Device Mode \\
\hline 1 & 1 & External Clock Mode \\
\hline 1 & 0 & Internal Clock Mode \\
\hline 0 & 1 & Fast Power-Down Mode \\
\hline 0 & 0 & Full Power-Down Mode \\
\hline
\end{tabular}

Table 7. Hard-Wired Shutdown and Compensation Mode

\begin{tabular}{|cll|}
\hline $\begin{array}{c}\text { SHDN } \\
\text { State }\end{array}$ & $\begin{array}{l}\text { Device } \\
\text { Mode }\end{array}$ & $\begin{array}{l}\text { Reference-Buffer } \\
\text { Compensation }\end{array}$ \\
\hline 1 & Enabled & Internal Compensation \\
\hline Floaking & Enabled & External Compensation \\
\hline 0 & Full Power-Down & N/A \\
\hline
\end{tabular}




\section{Low-Power, 8-Channel, Serial 12-Bit ADCs}

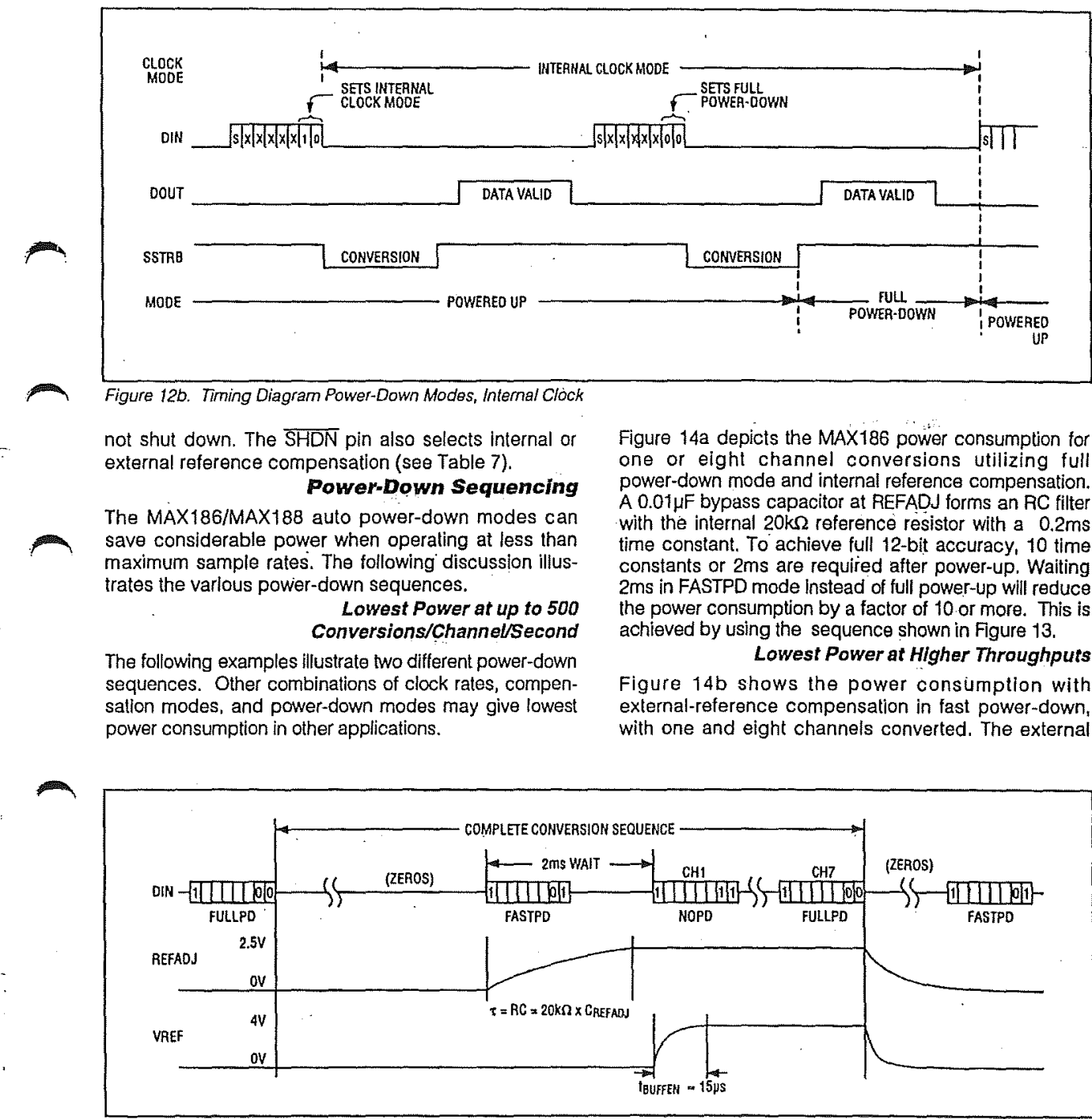

Figure 13. MAX186 FULLPD/FASTPD Power-Up Sequence 


\section{Low-Power, 8-Channel, Serial 12-Bit ADCs}

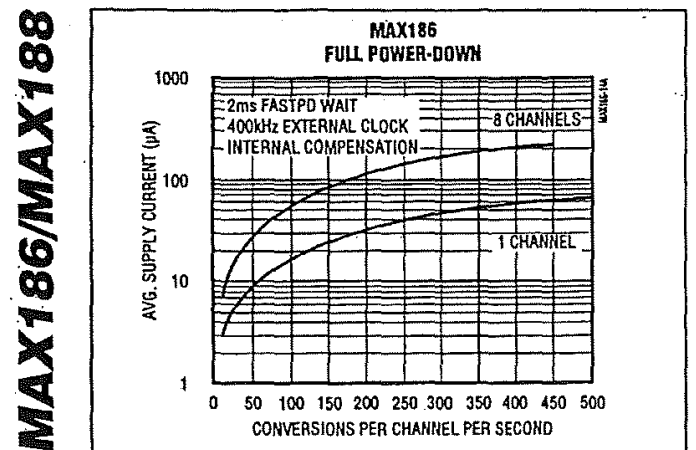

Figure 14a. MAX186 Supply Current vs. Sample Rate/Second, FULLPD, $400 \mathrm{kHz}$ Clock

$4.7 \mu \mathrm{F}$ compensation requires a $50 \mu \mathrm{s}$ wait after power-up, accomplished by 75 lole clocks after a dummy conversion. This circuit combines fast multi-channel conversion with lowest power consumption possible. Full power-down mode may provide increased power savings in applications where the MAX186/MAX188 are inactive for long periods of tirne, bul where intermittent bursts of high-speed conversions are required. .

\section{Extemal and Internal References}

The MAX186 can be used with an internal or external reference, whereas an external reference is required for the MAX188. Diode D1 shown in the Typlcal Operating Circuit ensures correct start-up. Any standard signal diode can be used. For both parts, an external reference can either be connected directly at the VREF terminal or at the REFADJ pin.

An internal buffer is designed to provide $4.096 \mathrm{~V}$ at VREF for both the MAX186 and MAX188. The MAX186's internally trimmed $2.46 \mathrm{~V}$ reference is buffered with a gain of 1.678 . The MAX188's buffer is trimmed with a buffer gain of 1.638 lo scale an external $2.5 \mathrm{~V}$ reference at REFADJ to $4.096 \mathrm{~V}$ at VREF.

\section{MAX186 Internal Reference}

The full-scale range of the MAX 186 with internal reference is $4.096 \mathrm{~V}$ with unipolar inputs, and $\pm 2.048 \mathrm{~V}$ with bipolar inputs. The internal reference voltage is adjustable to $\pm 1.5 \%$ with the Reference-Adjust Circuit of Figure 17.

External Reference

With both the MAX186 and MAX188, an external reference can be placed at either the input (REFADJ) or the

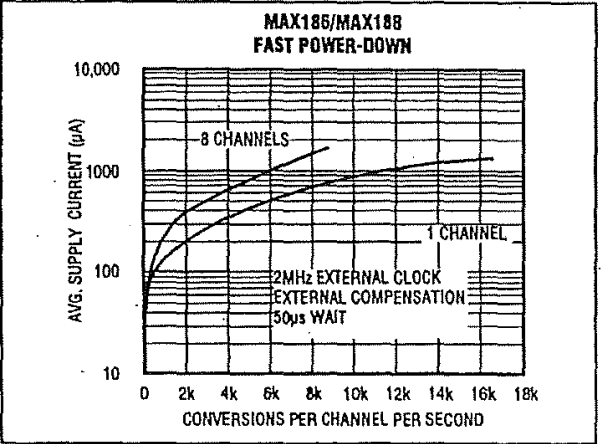

Figure 14b. MAX186/MAX188 Supply Current vs. Sample Rate/Second, FASTPD, 2MHz Clock

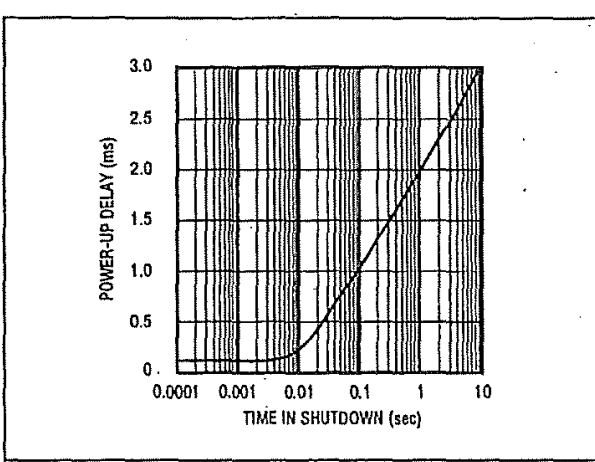

Figure 14c. Typical Power-Up Delay vs. Time in Shutdown

output (VREF) of the internal buffer ampliffer. The REFADJ input impedance is typically $20 \mathrm{k} \Omega$ for the MAX186 and higher than $100 \mathrm{k} \Omega$ for the MAX188, where the internal reference is omitted. At VREF, the input impedance is a minimum of $12 \mathrm{k} \Omega$ for DC currents. During conversion, an external relerence at VREF must be able to deliver up to $350 \mu \mathrm{A}$ DC load current and have an output impedance of $10 \Omega$ or less. If the reference has higher output impedance or is noisy, bypass it close to the VREF pin with a $4.7 \mu F$ capacitor.

Using the buffered REFADJ input avoids external buffering of the reference. To use the direct VREF input, disable the internal buffer by tying REFADJ to $V_{D D}$. 


\section{Low:Power, 8-Channel, Serial 12-Bit ADCs}

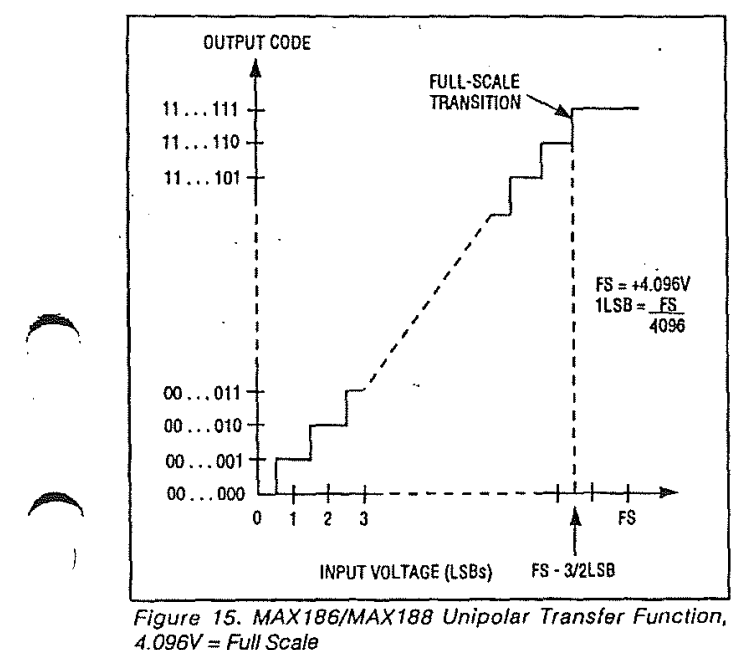

$4.096 \mathrm{~V}=$ Full SCale

Transfer Function and Gain Adjust

Figure 15 depicts the nominal, unipolar input/output (I/O) transfer function, and Figure 16 shows the bipolar input/output transfer function. Code transitions occur halfway between successive integer LSB values. Output coding is binary with $1 \mathrm{LSB}=1.00 \mathrm{mV}$ $(4.096 \mathrm{~V} / 4096)$ for unipolar operation and $1 \mathrm{LSB}=$ $1.00 \mathrm{mV}((4.096 \mathrm{~V} / 2-4.096 \mathrm{~V} / 2) / 4096)$ for bipolar operation.

Figure 17, the MAX186 Reference-Adjust Circuit, shows how to adjust the ADC gain in applications that use the internal reference. The circuit provides $\pm 1.5 \%$ ( $\pm 65 \mathrm{LSBS}$ ) of gain adjustment range.

Layout, Grounding, Bypassing

For best performance, use printed circuit boards. Wire-wrap boards are not recommended. Board layout should ensure that digital and analog signal lines are separated from each other. Do not run analog and digital (especially clock) lines parallel to one another, or digital lines underneath the ADC package.

Figure 18 shows the recommended system ground connections. A single-point analog ground ("star" ground point) should be established at AGND, separate from the logic ground. All other analog grounds and DGND should be connected to this ground. No other digital system ground should be connected to

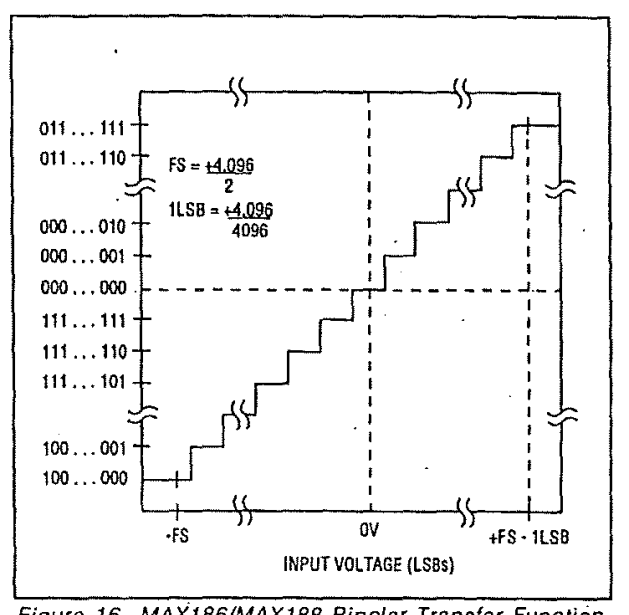

Figure 16. MAX186/MAX188 Bipolar Transfer Function $\pm 4.096 \mathrm{~V} / 2=$ Full Scale

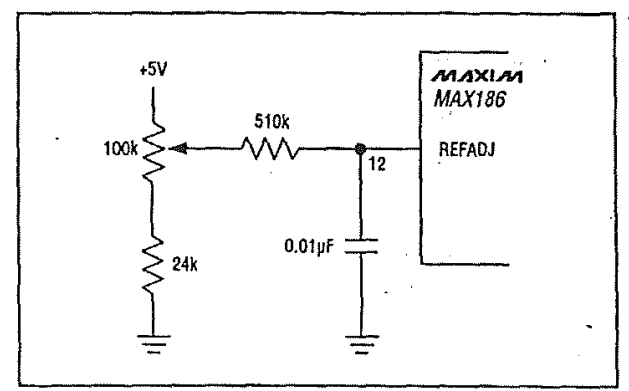

Figure 17. MAX186 Reference-Adjust Circuit

this single-point analog ground. The ground return to the power supply for this ground should be low impedance and as short as possible for noise-free operation.

High-frequency noise in the $V_{D D}$ power supply may affect the high-speed comparator in the ADC. Bypass these supplies to the single-point analog ground with $0.1 \mu \mathrm{F}$ and $4.7 \mu \mathrm{F}$ bypass capacitors close to the MAX186/MAX188. Minimize capacitor lead lengths for best supply-noise rejection. If the $+5 \mathrm{~V}$ power supply is very nolsy, a $10 \Omega$ resistor can be connected as a lowpass filler, as shown in Figure 18. 


\section{Low-Power, 8-Channel, Serial 12:Bit ADCs}

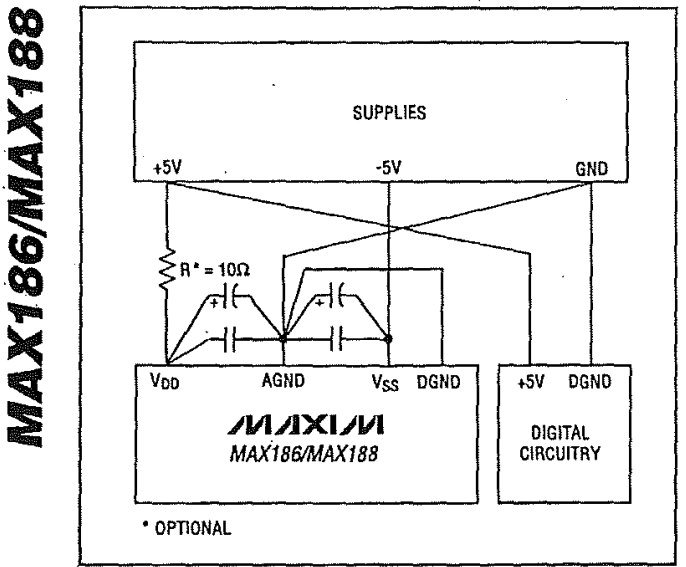

Figure 18. Power-Supply Grounding Connection
High-Speed Digital Interfacing with OSPI The MAX186/MAX188 can interface with QSPI at high throughput rates using the clrcuit in Figure 19. This QSPI circuit can be programmed to do a conversion on each of the eight channels. The result is stored in memory without taxing the CPU since QSPI incorporates its own micro-sequencer. Figure 19 depicts the MAX186, but the same circuil could be used with the MAX188 by adding an external reference to VREF and connecting REFADJ to $V_{D D}$. Figure 20 details the code that sets up QSPI for autonomous operation. In external clock mode, the MAX186/MAX188 perform a single-ended, unipolar conversion on each of their eight analog input channels. Figure 21, QSPI Assembly-Code Timing, shows the timing associated with the assembly code of Figure 20. The first byte clocked into the MAX186/MAX188 is the control byte, which triggers the first conversion on $\mathrm{CHO}$. The last two bytes clocked into the MAX186/MAX188 are all zero and clock out the results of the $\mathrm{CH} 7$ conversion.

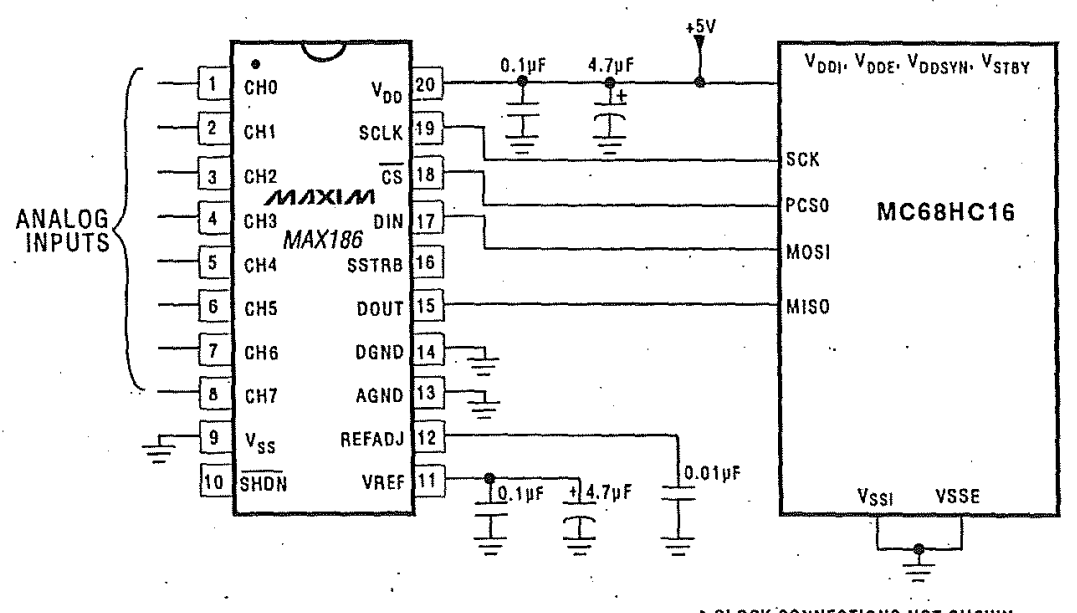

- CLOCX CONNECTIONS NOT SHOWN

Figure 19. MAX186 QSPI Connection 


\section{LowiPower, 8-Channel, Serial 12.Bit ADCs}

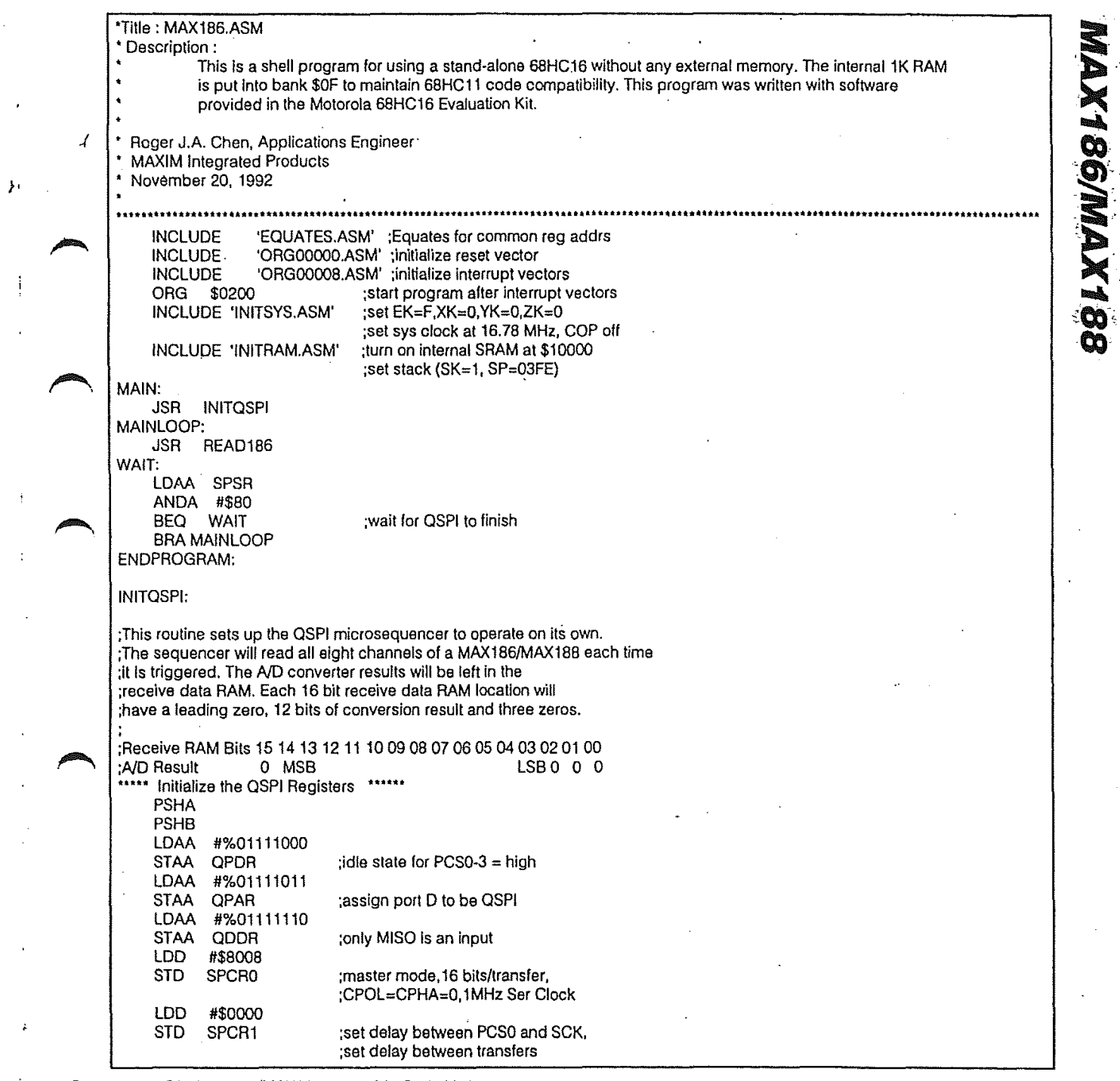

Figure 20. MAX186/MAX188 Assembly-Codo Listing 


\section{Low-Power, 8-Channel, Serial 12-Bit ADCs}

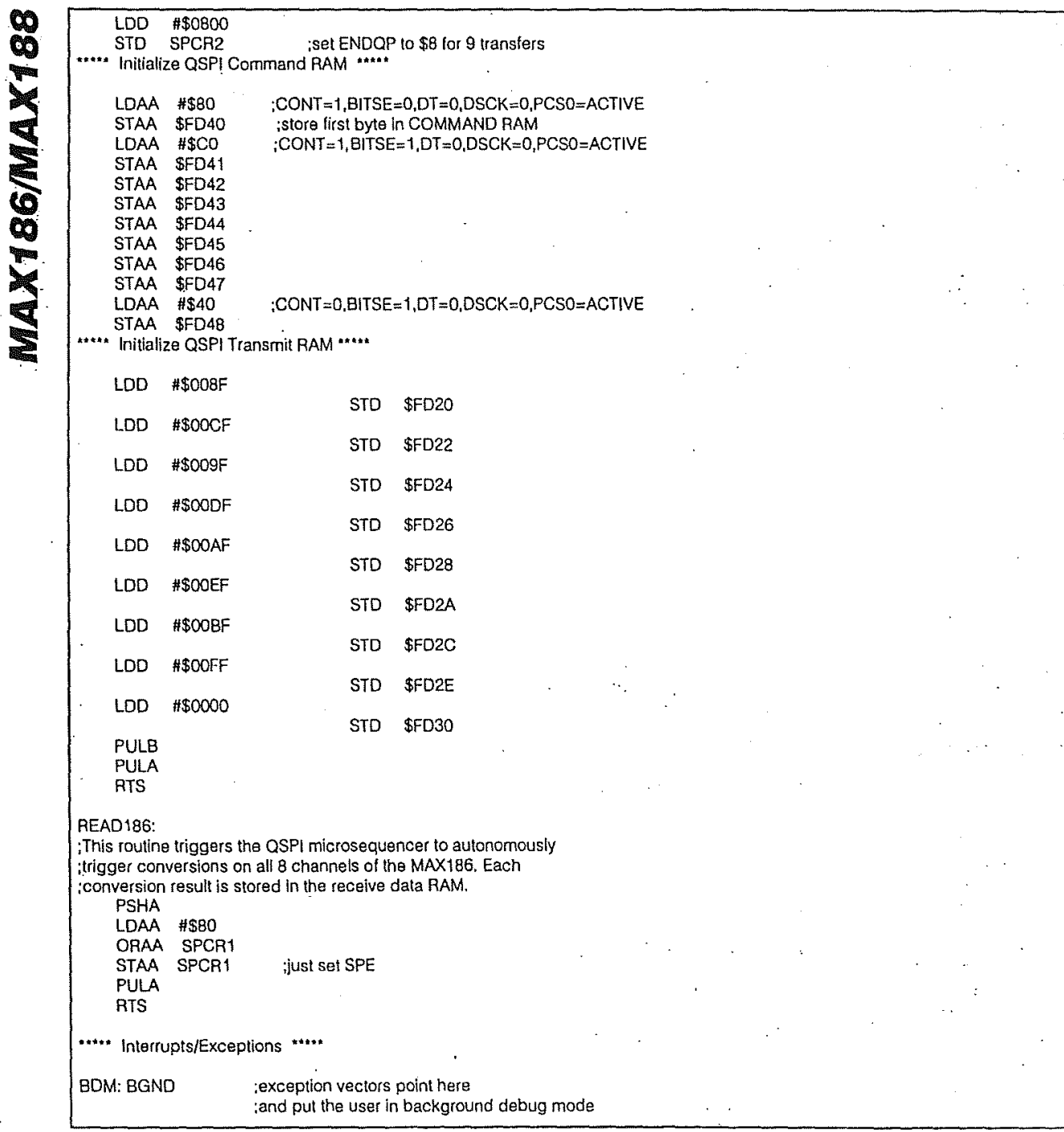

Figure 20. MAX186/MAX188 Assembly-Code Listing (continued)

22 


\section{Low-Power, 8-Channel, Serial 12-Bit ADCs}

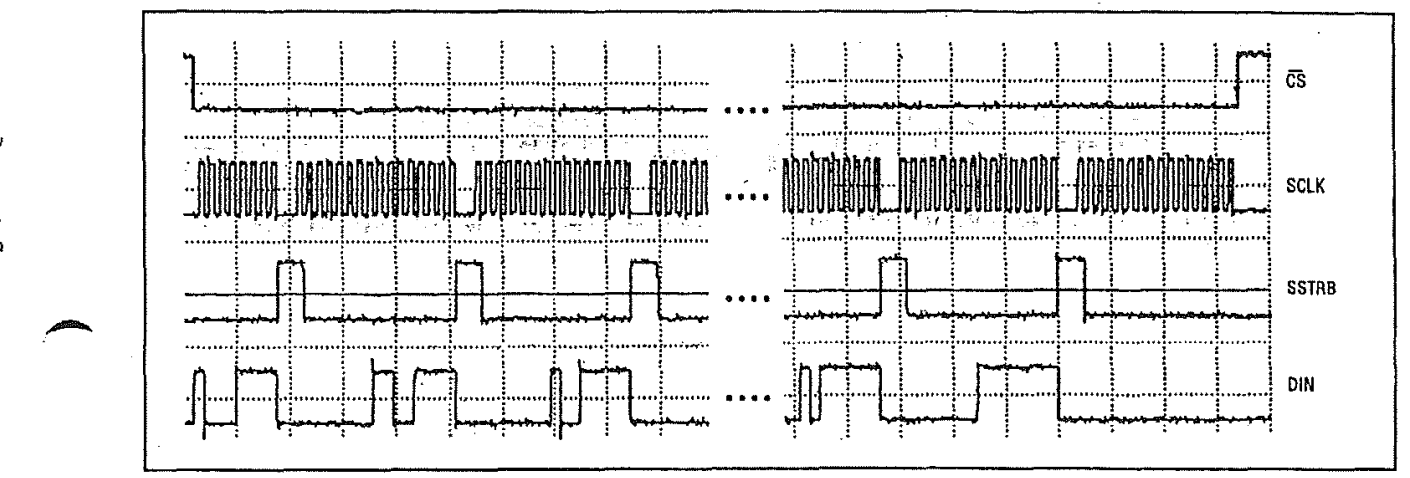

Figure 21. OSPI Assembly-Code Timing

TMS320C3x to MAX186 Interface

Figure 22 shows an application circuit to interface the MAX $186 /$ MAX 188 to the TMS320 in external clock mode. The timing diagram for this inlerface circuit is shown in Figure 23.

Use the following steps to Inltiate a conversion in the MAX186/MAX188 and to read the results:

1) The TMS320 should be configured with CLKX (transmil clock) as an active-high output clock and CLKR (TMS320 receive clock) as an active-high input clock. CLKX and CLKA of the TMS320 are tied together with the SCLK input of the MAX186/MAX188.

2) The MAX186/MAX188 $\overline{C S}$ is driven low by the XF VO port of the TMS320 to enable data to be clocked into DIN of the MAX186/MAX188.

3) An 8-bit word ( $1 X X X X X 11)$ should be written to the MAX186/MAX188 to initiate a conversion and place the device into external clock mode. Refer to Table 2 to select the proper XXXXX bit values for your specific application.

4) The SSTRB output of the MAX186/MAX188 is monitored via the FSR input of the TMS320. A falling edge on the SSTRB output indicates that the conversion is in progress and data is ready to be received from the MAX186/MAXi88.

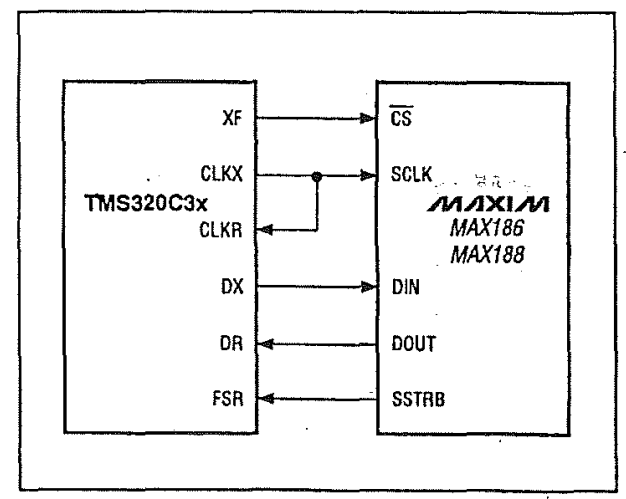

Figure 22. MAX186/MAX188 to TMS320 Serial Interface

5) The TMS320 reads in one data bit on each of the next 16 rising edges of SCLK. These data bits represent the 12-bil conversion result followed by four trailing bits, which should be ignored.

6) Pull $\overline{C S}$ high to disable the MAX186/MAX188 until the next conversion is initiated. 
Low-Power, 8-Channel, Serial 12-Bit ADCs

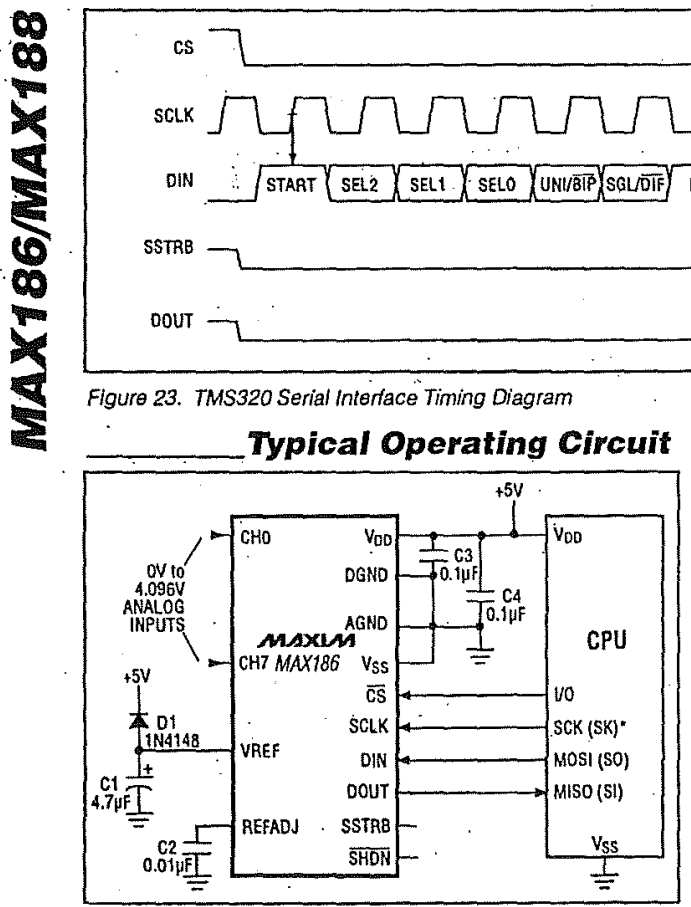

\section{_Ordering Information (continued)}

\begin{tabular}{|c|c|c|}
\hline PART & TEMP. RANGE & PIN-PACKAGE \\
\hline MAX188_CPP & $0^{\circ} \mathrm{C} 10+70^{\circ} \mathrm{C}$ & 20 Plastic DIP. \\
\hline MAX188_CWP & $0^{\circ} \mathrm{C} 10+70^{\circ} \mathrm{C}$ & 2050 \\
\hline MAX188_CAP & $0^{\circ} \mathrm{C} 10+70^{\circ} \mathrm{C}$ & 20 SSOP \\
\hline MAX188DCD & $0^{\circ} \mathrm{C} 10+70^{\circ} \mathrm{C}$ & Dice* \\
\hline MAX188_EPP & $-40^{\circ} \mathrm{C} 10+85^{\circ} \mathrm{C}$ & Plastic DIP \\
\hline MAX18B_EWP & $-40^{\circ} \mathrm{C}$ to $+85^{\circ} \mathrm{C}$ & 2050 \\
\hline MAX188_EAP & $-40^{\circ} \mathrm{C}$ to $+85^{\circ} \mathrm{C}$ & 20 SSOP \\
\hline MAX188_MJP. & $-55^{\circ} \mathrm{C}$ to $+125^{\circ} \mathrm{C}$ & 20 CERDIP: \\
\hline PART & TEMP. RANGE & BOARD TYPE \\
\hline MAX186EVKIT-DIP & $0^{\circ} \mathrm{C}$ to $+70^{\circ} \mathrm{C}$ & Through-Hole \\
\hline
\end{tabular}

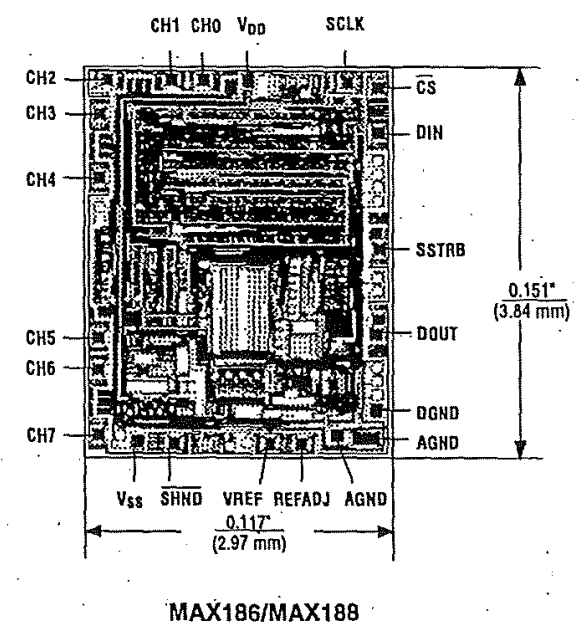

TRANSISTOR COUNT: 2278

SUBSTRATE CONNECTED TO VDD.

+ NOTE: Parts are offered in grades A, B, $C$ and D (grades defined in Electrical Characteristics). When ordering, please specify grade. - Dice are specified at $+25^{\circ} \mathrm{C}$, DC parameters only.

- Contact factory for availability and processing to MIL-STD-883.

Maxim cannot assume responsibility for use of any circuity other than circuity entirely embodied in a Maxim product. No ctrcuit palent licenses. are implied, Maxim reserves the right to change the circuity and specifications without notice al any time.

$24 \ldots$ Maxim Integrated Products, 120 San Gabriel Drive, Sunnyvale, CA 94086 (408) 737.7600 (c) 1995 Maxim integrated Products Printed USA MAXIM is a registered trademark of Maxim Integrated Products. 


\section{D3. HCPL-7800 Isolation Amplifier}

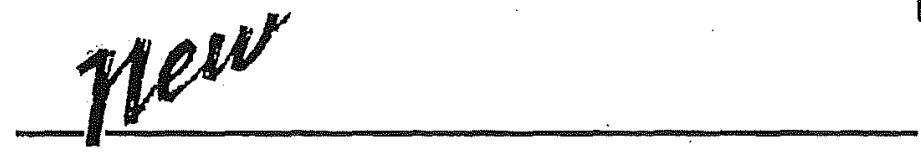

\section{High CMR Isolation Amplifier}

\section{Technical Data}

\section{Features}

- $15 \mathrm{kV} / \mu \mathrm{s}$ Common-Mode Rejection at $V_{C M}=1000 V^{*}$

- Compact, Auto-Insertable Standard 8-pin DIP Package

- $4.6 \mu \mathrm{V} /{ }^{\circ} \mathrm{C}$ Offset Drift $\mathrm{vs.}$

Temperature

- $0.9 \mathrm{mV}$ Input Offset Voltage

- $85 \mathrm{kHz}$ Bandwidth

- $0.1 \%$ Nonlinearity

- Worldwide Safety Approval: UL 1577, VDE 0884 and CSA

- Advanced Sigma-Delta $(\Sigma \Delta)$

A/D Converter Technology

- Fully Differential Circuit

Topology

- $1 \mu \mathrm{m}$ CMOS IC Technology

\section{Applications}

- Motor Phase Current

Sensing

- General Purpose Industrial

Current Sensing

- High-Voltage Power

Source Voltage Monitoring

The terms common-mode rejection (CMR) and isolation-mode rejection (IMR) are used interchangeably throughout this data sheet.
- Switch-Mode Power Supply Signal Isolation

- General Purpose Analog Signal Isolation

- Transducer Isolation

\section{Description}

The HCPL-7800 high CMR isolation amplifier provides a unique combination of features ideally suited for motor control circuit designers. The product provides the precision and stability needed to accurately monitor motor current in highnoise motor control environments, providing for smoother. control (less "torque ripple") in various types of motor control applications.

This product paves the way for a smaller, lighter, easier to produce, high noise rejection, low cost solution to motor current sensing. The product can also be used for general analog signal issolation applications requiring high accuracy, stability and linearity under similarly severe noise condi-
HCPL-7800

HCPL:7800A

HCPL-7800B

CAUTION: The small device geometries inherent to the design of this CMOS component increase the component's susceptibility to damage from electrostatic discharge (ESD). It is advised that normal static precautions be taken in handling and assembly of this component to prevent damage and/or degradation which may be induced by ESD. 
shielded detector using our patented "light-pipe" optocoupler packaging technology.

Together, these features deliver unequaled isolation-mode noise rejection, as well as excellent offset and gain accuracy and stability over timie and temperature. All in a compact; autoinsertable, industry standard 8-pin.DIP package that meets worldwide regulatory safety standards (gull-wing surface mount option \#300 also available).
Regulatory Information The HCPL-7800 has been approved by the following organizations:

UL

Approved under UL1577, component recognition FILE E55361

VDE

Approved according to VDE 0884/08.87

Can be used for safe electrical separation between AC mains and SELV (safety extra-low voltage) in equipment according to the following specifications:

DIN VDE 0804/05.89

DIN VDE 0160/05.8

Reference voltage (VDE $0110 \mathrm{~b}$

Tab 4): $650 \mathrm{Vac}$

\section{CSA}

Approved under CSA 22.2 No. 0.

General Requirements,

Canadian Electrical Code, Part

II; and CSA Component

Acceptance Notice \#5, File CA 88324.

\section{VDE 0884 Insulation Characteristics}

\begin{tabular}{|c|c|c|c|}
\hline Description & Symbol & Characteristic & Unit \\
\hline $\begin{array}{l}\text { Installation classification per } \mathrm{DIN} \text { VDE } 0109 / 12.83 \text {, Table } 1 \\
\text { for rated mains voltage } \leq 300 \mathrm{~V}_{\mathrm{RMS}} \\
\text { for rated mains voltage } \leq 600 \mathrm{~V}_{\mathrm{RMS}}\end{array}$ & & $\begin{array}{l}\text { I-IV } \\
\text { I-III }\end{array}$ & \\
\hline Climatic Classification & & $40 / 85 / 21$ & \\
\hline Pollution Degree (DIN VDE 0109/12.83)* & & 2 & \\
\hline Maximuim Working Insulation Voltage & $\mathrm{V}_{\text {IORM }}$ & $600^{\circ}$ & $\mathrm{V}_{\mathrm{RMS}}$ \\
\hline 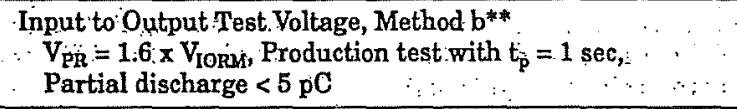 & $V_{P R}$ & 960 & $V_{R M S}$ \\
\hline $\begin{array}{l}\text { Input to Output Test Voltage, Method } \mathrm{a}^{* *} \\
\mathrm{~V}_{\mathrm{pR}}=1.2 \times \mathrm{V}_{\mathrm{IORM}}, \text { Type and sample test; } \mathrm{t}_{\mathrm{p}}=60 \mathrm{sec},: \\
\text { Partial discharge }<5 \mathrm{pC}\end{array}$ & $V_{\mathrm{PR}}$ & 720 & $V_{\text {RMS }}$ \\
\hline $\begin{array}{l}\text { Highest Allowable Overvoltage } \\
\text { (Transient Overvoltage, } t_{\mathrm{TR}}=10 \mathrm{sec} \text { ) }\end{array}$ & $\overline{V_{T R}}$ & 6000 . & $V_{\text {PEAK }}$ \\
\hline $\begin{array}{l}\text { Safety-limiting values (Maximum values allowed in the } \\
\text { event of a failure, also see Figure 27) } \\
\text { Case Temperature } \\
\text { Input Power } \\
\text { Output Power }\end{array}$ & $\begin{array}{l}\mathrm{T}_{\mathrm{SI}} \\
\mathrm{P}_{\mathrm{SI} \text {, Input }} \\
\mathrm{P}_{\mathrm{SI}, \text { Output }}\end{array}$ & $\begin{array}{l}175 \\
80 \\
250\end{array}$ & ${ }^{\circ} \mathrm{C}$ \\
\hline Insulation Resistance at $\mathrm{T}_{\mathrm{SI}}, \mathrm{V}_{\mathrm{IO}}=500 \mathrm{~V}$ & $R_{1 S}$ & $\geq 10 \mathrm{E} 11$ & Ohm \\
\hline
\end{tabular}

-This part may also be used in Pollution Degree 3 environments where the rated mains voltage is $\leq 300 V_{\text {aus }}$ (per DIN VDE 0109/12,83).

**Refer to the front of the optocoupler section of the current Optoelectronics Designer's Catalog for a more detailed description of VDE 0884 and other product safety regulations. 
Insulation Related Specifications

\begin{tabular}{|l|c|c|c|l|}
\hline \multicolumn{1}{|c|}{ Parameter } & Symbol & Value & Units & \multicolumn{1}{|c|}{ Conditions } \\
\hline $\begin{array}{l}\text { Min. External air gap } \\
\text { (clearance) }\end{array}$ & L(IO1) & $>7$ & $\mathrm{~mm}$ & $\begin{array}{l}\text { Measured from input terminals to } \\
\text { output terminals }\end{array}$ \\
\hline $\begin{array}{l}\text { Min. External tracking path } \\
\text { (creepage) }\end{array}$ & $\mathrm{L}(\mathrm{IO2})$ & $\begin{array}{c}8.0 \\
\vdots\end{array}$ & $\mathrm{mm}$ & $\begin{array}{l}\text { Measured from input terminals to } \\
\text { output terminals }\end{array}$ \\
\hline $\begin{array}{l}\text { Min. Internal plastic gap } \\
\text { (clearance) }\end{array}$ & & 0.5 & $\mathrm{~mm}$ & $\begin{array}{l}\text { Through insulation distance } \\
\text { conductor to conductor }\end{array}$ \\
\hline Tracking resistance & CTI & 175 & Volts & DIN IEC 112/VDE 0303 Part 1 \\
\hline $\begin{array}{l}\text { Isolation Group } \\
\text { (per DIN VDE 0109) }\end{array}$ & $\vdots$ & $\mathrm{IIra}$ & $\cdots$ & Material Group DIN VDE 0109 \\
\hline
\end{tabular}

\section{Package Outline Drawings}

Standard DIP. Package

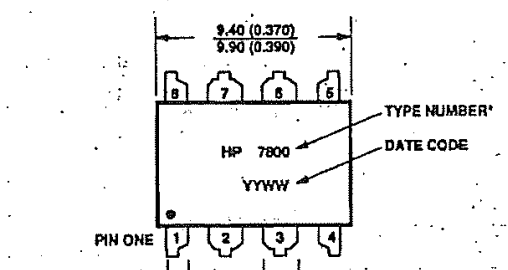

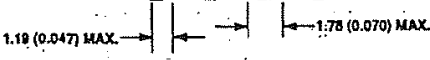
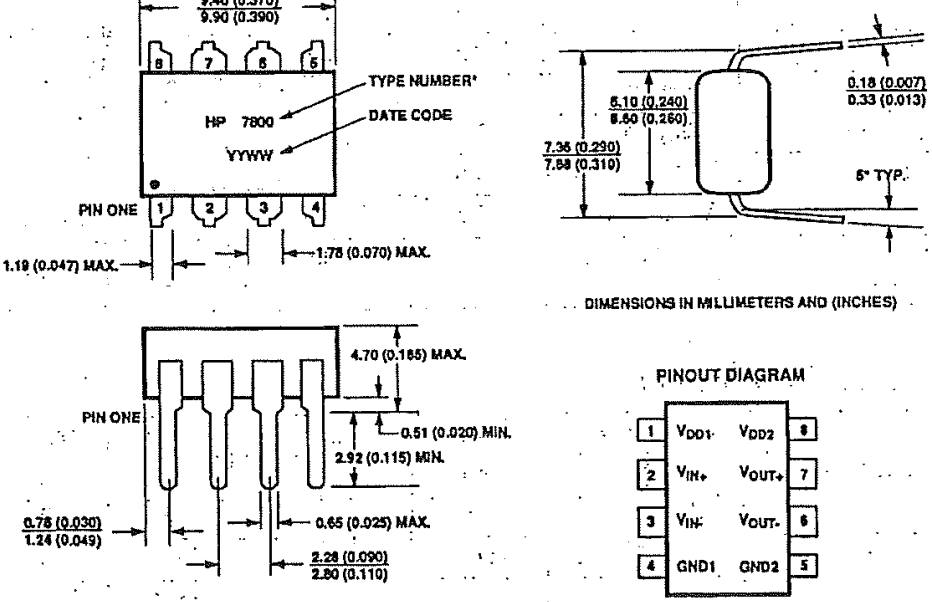

OMMENSIONS IN MALUKETEPS ANO (INCHES)

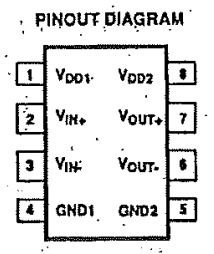

TTPE NUMBEER FOR : HCPL-TO00 * 7000

HCPLLTH00A $=7800 A$
HCPL $78000=78008$

Gull Wing Surface Mount Option $900^{*}$
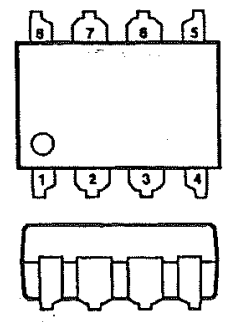

- heFer to opton 300 Data sheet FOR MORE IAFORMATION.

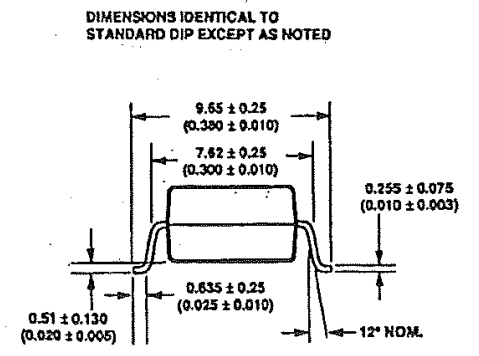

$6-223$ 


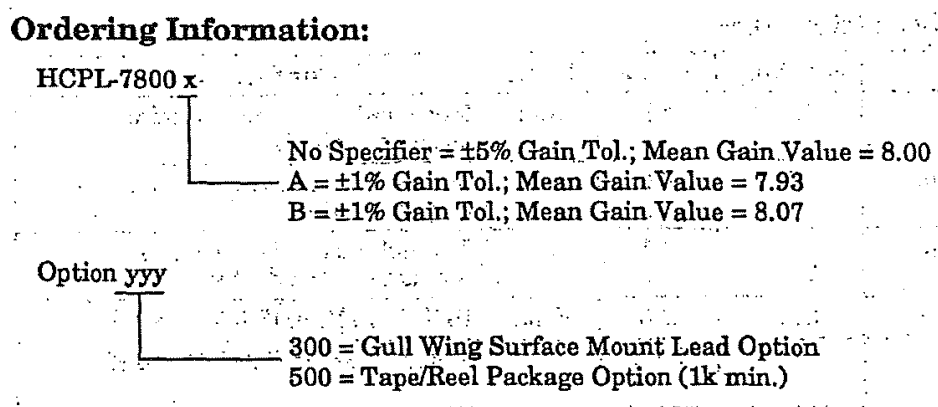

Absolute Maximum Ratings

\begin{tabular}{|c|c|c|c|c|c|}
\hline Parameter & Symbol & Min. & $\operatorname{Max}$ & Unit & Note \\
\hline Storage Temperature & $\mathrm{T}_{\mathrm{S}}$ & .55 & 125 & ${ }^{\circ} \mathbf{C}$ & \\
\hline Ambient Operating Temperature & $\mathrm{T}_{\mathrm{A}}$ & -40 & 100 & ${ }^{\circ} \mathrm{C}$ & \\
\hline Supply Voltages & $\mathrm{V}_{\mathrm{DD1}}, \mathrm{V}_{\mathrm{DD} 2}$ & 0 & 5.5 & $\mathrm{~V}$ & \\
\hline Steady-State Input Voltage & \multirow[t]{2}{*}{$\mathrm{V}_{\mathrm{IN}+}, \mathrm{V}_{\mathrm{IN}}$} & -2.0 & \multirow[t]{2}{*}{$\mathrm{V}_{\mathrm{DDI}}+0.5$} & \multirow[t]{2}{*}{$\overline{\mathrm{V}}$} & \\
\hline 2 Second Transient Input: Voltage & & -6.0 & & & \\
\hline Output Voltages & $V_{\text {OUT }+}, V_{\text {OUT }}$ & -0.5 & $\mathrm{~V}_{\mathrm{DD} 2}+0.5$ & $\mathrm{~V}$ & \\
\hline $\begin{array}{l}\text { Lead Solder Temperature } \\
\text { (1.6 mm below seating plane, } 10 \mathrm{sec} \text {.) }\end{array}$ & $T_{L S}$ & 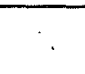 & 260 & ${ }^{\circ} \mathrm{C}$ & $\because 1$ \\
\hline
\end{tabular}

Recommended Operating Conditions

\begin{tabular}{|l|c|c|c|c|c|}
\hline \multicolumn{1}{|c|}{ Parameter } & Symibol & Min. & Max. & Unit & Note \\
\hline Ambient Operating Temperature & $\mathrm{T}_{\mathrm{A}}$ & -40 & 85 & ${ }^{\circ} \mathrm{C}$ & 2 \\
\hline Supply Voltages & $\mathrm{V}_{\mathrm{DD1}}, \mathrm{V}_{\mathrm{DD} 2}$ & 4.5 & 5.5 & $\mathrm{~V}$ & $\mathbf{3}$ \\
\hline Input Voltage & $\mathrm{V}_{\mathrm{IN}}, \mathrm{V}_{\mathrm{IN}}$ & -200 & 200 & $\mathrm{mV}$ & 4 \\
\hline Output Current & $\mathrm{N}_{\mathrm{IOI}}$ & & 1 & $\mathrm{~mA}$ & 5 \\
\hline
\end{tabular}


DC Electrical Specifications

DC Electrical Specifications
All specifications and figures are at the nominal operating condition of $\mathrm{V}_{\mathrm{IN}+}=0 \mathrm{~V}, \mathrm{~V}_{\mathrm{IN}-}=0, \mathrm{~V}, \mathrm{~T}_{\mathrm{K}}=25^{\circ} \mathrm{C}$,
$\mathrm{V}_{\mathrm{DDI}}=5.0 \mathrm{~V}$, and $\mathrm{V}_{\mathrm{DDg}}=5.0 \mathrm{~V}$, unless othervise noted.

\begin{tabular}{|c|c|c|c|c|c|c|c|c|}
\hline Parameter & Symbol & Min.- & Typ. & Max. & Unit & $\begin{array}{l}\text { Test } \\
\text { Conditions }\end{array}$ & Fig. & Note \\
\hline Input Offset Voltage & $V_{O B}$ & -1.8 & .0 .9 & 0.0 & $\mathrm{mV}$ & $\therefore$ & 1 & \\
\hline $\begin{array}{l}\text { Input Offset Drift v8. } \\
\text { Temperature }\end{array}$ & $\mathrm{dV} \mathrm{V}_{\mathrm{g}} / \mathrm{d} \mathrm{T}$ & $\cdots$ & -2.1 & & $\mu \mathrm{V} /{ }^{\circ} \mathrm{C}$ & $\cdot$. & 1,2 & 6. \\
\hline $\begin{array}{l}\text { Abs. Value of Input } \\
\text { Offset Drift vs. Temperature }\end{array}$ & $\mid \mathrm{dV} \mathrm{Vg}_{\mathrm{g}} / \mathrm{dT}$ & & 4.6 & & $\mu \mathrm{V} /{ }^{\circ} \mathrm{C}$ & & 1 & 7 \\
\hline Input Offset Drift vs. $V_{D D 1}$ & $d V_{0 S} / d V_{D D 1}$ & & 30 & & $\mu \nabla / V$ & & 1,3 & 8 \\
\hline Trput Offset Drift vs. $\bigvee_{\mathrm{DD} 2}$ & $d V_{09} / \mathrm{dV}_{\mathrm{DD} 2}$ & & .40 & & $\mu \mathrm{V} / \mathrm{V}$ & & 1,4 & 9 \\
\hline Gain ( $\pm 5 \%$ Tol.) & G. & 7.61 & 8.00 & 8.40 & & \multirow{10}{*}{$\begin{array}{c}-200<Y_{\mathrm{in}+}<200 \mathrm{mV} \\
\cdots\end{array}$} & 1,5 & \multirow[t]{3}{*}{10.} \\
\hline Gain - A Version ( $(1 \%$ Tol.) & $\mathrm{G}_{\mathrm{A}}$ & 7.85 & 7.83 & 8.01 & & & & \\
\hline Gain - B Version ( $\pm 1 \%$ Tol. $)$ & $\mathrm{G}_{\mathrm{B}}$ & 7.88 & 8.07 & 8.15 & & & & \\
\hline Gain:Drift vis: Temperature & $\mathrm{dG} / \mathrm{dT}$ & $\because$ & 0.001 & & $\% \circ \mathrm{C}$ & & 5,6 & $\overline{11}$ \\
\hline $\begin{array}{l}\text { Abs. Value of Galn Drift vs. } \\
\text { Temperature }\end{array}$ & |dG/dT & $\therefore$ & 0.001 & & $\% \%^{\circ} \mathrm{C}$ & & 5 & 12 \\
\hline Gain Drift vs; $V_{D D 1}$ & $\mathrm{dG} / \mathrm{dV}_{\mathrm{DD1}}$ & & 0.21 & & $\% O N$ & & 5,7 & 13 : \\
\hline Gain Drift vs. $V_{D D_{2}}$ & $\mathrm{dG} / \mathrm{d} V_{\mathrm{DD} 2}$ & & -0.06 & & EN & & 5,8 & 14. \\
\hline 200 mV Nonlinearity & $\mathrm{NL}_{200}$ & & 0.2 & 0.35 & $\Phi$ & & 5,9 & 15 \\
\hline $\begin{array}{l}200 \mathrm{mV} \text { Nonlinearity Drift. } \\
\text { ys: Temperature }\end{array}$ & $\mathrm{dNL}_{20 \mathrm{~d}} / \mathrm{dT}$ & & -0.001 & $i \ldots$ & $90 \mathrm{pts} /{ }^{\circ} \mathrm{C}$ & & 5,10 & 16 \\
\hline $\begin{array}{l}200 \mathrm{mV} \text { Nonlínearity Drift } \\
\text { VB. V VDI }\end{array}$ & $\mathrm{dNL}_{200} / \mathrm{dV} \mathrm{V}_{\mathrm{DD1}}$ & & 0.005 & & optan & & 5,111 & 17 \\
\hline $\begin{array}{l}200 \mathrm{mV} \text { Nonlinearity Drift } \\
\text { vs. } \mathrm{V}_{\mathrm{DD} 2}^{\prime}\end{array}$ & $d \mathrm{NL}_{200} / \mathrm{d} \mathrm{V}_{\mathrm{DD}}$ & $\therefore$ & -0.007 & $\therefore$ & $\% \mathrm{ptg} / \mathrm{V}$ & ${ }_{0}$ & 5,12 & 18 \\
\hline $100 \mathrm{mV}$ Nonlinearity & $\mathrm{NL}_{100}$ & & 0.1 & 0.25 & $\%$ & $-100<\mathrm{V}_{\mathrm{Ni}+}<100 \mathrm{mV}$ & $5 ; 13$ & 19 \\
\hline $\begin{array}{l}\text { Maximum Input Voltage } \\
\text { Befotote Output Clipping }\end{array}$ & $=\left|V_{\mathrm{RN}_{+}}\right|_{\text {max }}$. & & 300 & & $\mathrm{mV}$ & & .14 & \\
\hline Average Input Bias Current & $\mathrm{I}_{\mathrm{IN}}$ & & .670 & & $\mathrm{nA}$ & & 15,16 & 20. \\
\hline $\begin{array}{l}\text { Input Bias Current } \\
\text { Temperature Coefficient. }\end{array}$ & $\mathrm{dI}_{\mathrm{IN}} / \mathrm{dT}$ & & 3 & 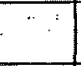 & $\mathrm{nA}{ }^{\mu} \mathrm{C}$ & & & \\
\hline Average Input Resistance & $\mathbf{R}_{\mathrm{IN}}$ & & 530 & & $\mathrm{k} \Omega$ & & 15 & 20 \\
\hline $\begin{array}{l}\text { Thput Resistancë } \\
\text { Temperature Coefficient }\end{array}$ & $d R_{i N} y d T$ & & 0.38 & & $90^{\circ} \mathrm{C}$ & & & \\
\hline $\begin{array}{l}\text { Input DC Common-Mode } \\
\text { Rejection Ratio }\end{array}$ & $C M R R_{I N}$ & & 72 & & $\bar{d} \overline{\mathrm{B}}$ & & & 21 \\
\hline Output Resistance & $R_{0}$ & & 11 & . & $\bar{\Omega}$ & $\therefore$ & 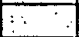 & $\therefore 5$ \\
\hline $\begin{array}{l}\text { Output Resistance } \\
\text { Temperature Coefficient }\end{array}$ & $\mathrm{dR} / \mathrm{d} T$ & $\therefore$ & 0.6 & & $\% 0^{\circ} \mathrm{C}$ & $\therefore$ & & $\because$ \\
\hline Output Low Voltage ... ... &. $\mathrm{V}_{\mathrm{OL}}$ & $\because$ & 1.18 & . & $\ldots V$ & $.1 V_{I N+} I=500 \mathrm{mV}$ & 14 & 22 \\
\hline Output High Voltage & $\overline{\mathrm{V}_{\mathrm{OH}}}$ & & 3.61 . & & $\bar{\nabla}$ & $\ldots$ & & \\
\hline $\begin{array}{l}\text { Output Common-Mode. } \\
\text { Voltage }\end{array}$ & $\mathrm{Y}_{\mathrm{OCM}}$ & 2.20 & 2.39 & 2.60 . & V & $\begin{array}{l}40^{\circ} \mathrm{C}<\mathrm{T}_{\mathrm{A}}<85^{\circ} \mathrm{C} \\
4.5 \mathrm{~V}<\mathrm{V}_{\mathrm{DD} 1}<5.5 \mathrm{~V}\end{array}$ & 14 & \\
\hline Input Supply Current & $\mathrm{I}_{\mathrm{DD} 1}$ & & 10.7 & 13.5 & $\overline{\mathrm{mA}}$ & & 17 & 23 \\
\hline Output Supply Current & $\mathrm{I}_{\mathrm{DD2}}$ & & 11.6 & 14.5 & $\mathrm{~mA}$ & $\begin{array}{l}\mathrm{V}_{\mathrm{IN}_{+}}=200 \mathrm{mV} \\
40^{\circ} \mathrm{C}<\mathrm{T}_{\mathrm{A}}<85^{\circ} \mathrm{C} \\
4.5 \mathrm{~V}<\mathrm{V}_{\mathrm{DD} 2}<5.5 \mathrm{~V}\end{array}$ & 18 & 24 \\
\hline $\begin{array}{l}\text { Output Short-Circuit } \\
\text { Current }\end{array}$ & $\mathrm{II}_{\mathrm{osa}} \mathrm{I}$ & & $\overline{9.3}$ & & $\mathrm{~mA}$ & $V_{\text {OUT }}=0 V_{\text {or }} V_{D D 2}$ & & 25 \\
\hline
\end{tabular}




\section{AC Electrical Specifications}

All specifications and figures are at the nominal operating condition of $\mathrm{V}_{\mathrm{IN}_{+}}=0 \mathrm{~V}, \mathrm{~V}_{\mathrm{IN} .}=0 . \mathrm{V}, \mathrm{T}_{\mathrm{A}}=25^{\circ} \mathrm{C}$, $\mathrm{V}_{\mathrm{DD1}}=5.0 \mathrm{~V}$, and $\mathrm{V}_{\mathrm{DD2}}=5.0 \mathrm{~V}$, unless otherwise noted.

\begin{tabular}{|c|c|c|c|c|c|c|c|c|}
\hline Parameter & Symbol & Min. & Typ. & Max. & Unit & Test Conditions & Fig. & Note \\
\hline $\begin{array}{l}\text { Riging Edge Isolation } \\
\text { Mode Rejection }\end{array}$ & $\mathrm{IMR}_{\mathbf{R}}$ & 10 & 25 . & & $\mathrm{kV} / \mathrm{\mu s}$ & \multirow[t]{2}{*}{$\nabla_{I M}=1 \mathrm{kV}$} & \multirow[t]{2}{*}{19,20} & \multirow[t]{2}{*}{26} \\
\hline $\begin{array}{l}\text { Falling Edge Isolation } \\
\text { Mode Rejection }\end{array}$ & $\mathrm{IMR}_{\mathbf{p}}$ & 10 & 15 & & $\mathrm{kV} / \mu \mathrm{s}$ & & & \\
\hline $\begin{array}{l}\text { Isolation Mode Rejection } \\
\text { Ratio at } 60 \mathrm{~Hz}\end{array}$ & IMRR & & $>140$ & & $\mathrm{~dB}$ & & 19 & 27. \\
\hline Propagation Delay to $10 \%$ & tip10 & & 2.0 & 3.3 & $\mu$ & \multirow{5}{*}{ 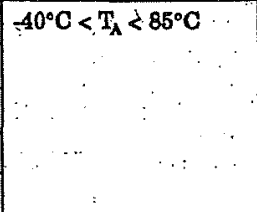 } & \multirow[t]{4}{*}{21,22} & \multirow{4}{*}{$\begin{array}{l}3 \\
\vdots \\
\vdots\end{array}$} \\
\hline Propagation Delay to 50\% & $t_{\text {PDoO }}$ & & 3.4 & 5.6 & $\mu \mathrm{s}$ & & & \\
\hline Propagation Delay to $90 \%$ & $t_{\mathrm{PD} P 0}$ & & 6.3 & 9.9 & $\mu \mathrm{s}$ & & & \\
\hline Rise/Fall Timo $(10 \%-90 \%)$ & $t_{R / P}$ & & 4.3 & 6.6 & $\mu$ & & & \\
\hline Bandwidth (-3 dB) & $f_{-a A B}$ & 50 & 85 & & $\mathbf{k H z}$ & & \multirow[t]{2}{*}{23,24} & \\
\hline Bandwidth $\left(-45^{\circ}\right)$ & $\mathbf{L}_{45:}$ & & 95: & & $\mathrm{kHz}$ & & & \\
\hline $\begin{array}{l}\text { RMS Input-Referred } \\
\text { Noise }\end{array}$ & $\mathrm{V}_{\mathrm{N}}$ & • & 300 & & $\mu \nabla_{\min }$ & Bandwidth $=100 \mathrm{kHz}$ & 25,26 & 28 \\
\hline Power Supply Rejection & PSR & & $\mathbf{5}$ & & $m V_{p k-p k}$ & & & 29 \\
\hline
\end{tabular}

\section{Package Characteristics}

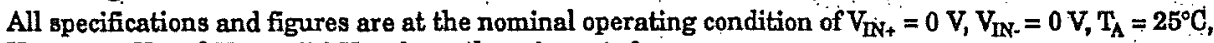
$V_{D D 1}=5.0 \mathrm{~V}$, and $V_{D D 2}=5.0 \mathrm{~V}$, unless otherwise noted.

\begin{tabular}{|c|c|c|c|c|c|c|c|c|c|}
\hline Parameter & Symbol & Min. & Typ. & $\operatorname{Max}:$ & Unit & \multicolumn{2}{|c|}{ Test Conditions } & Fig. & Note \\
\hline $\begin{array}{l}\text { Input-Output Insulation } \\
\text { Voltage }\end{array}$ & $\mathrm{V}_{\text {Iso }}$ & 3750 & & & $V_{\mathrm{rms}}$ & \multicolumn{2}{|c|}{$t=1 \mathrm{~min}, \mathrm{RH} \leq 50 \%$} & $\therefore$ & 30,31 \\
\hline \multirow[t]{2}{*}{ Input-Output Resistance } & \multirow[t]{2}{*}{$\mathbf{R}_{\text {to }}$} & $10^{12}$ & $10^{13}$ & & \multirow[t]{2}{*}{$\Omega$} & $T_{\Lambda}=25^{\circ} \mathrm{C}$ & \multirow[t]{2}{*}{$V_{L O}=500 V_{d e}$} & & \multirow[t]{2}{*}{30} \\
\hline & & $10^{11}$ & & & & $T_{A}=100^{\circ} \mathrm{C}$ & & & \\
\hline $\begin{array}{l}\text { Input-Output } \\
\text { Capacitance }\end{array}$ & $\mathrm{C}_{10}$ & & 0.7 & & $\mathrm{pF}$ & \multicolumn{2}{|l|}{$\mathrm{f}=1 \mathrm{MHz}$} & & 30 \\
\hline $\begin{array}{l}\text { Input IC Junction-to- } \\
\text { Case Thermal Resistance }\end{array}$ & $\theta_{\mathrm{Jd}}$ & & 96 & & ${ }^{\circ} \mathrm{C} / \mathrm{W}$ & & & \multirow[t]{2}{*}{32} \\
\hline $\begin{array}{l}\text { Output IC Junction-to- } \\
\text { Case Thermal Resistance }\end{array}$ & $\theta_{\text {jeo }}$ & 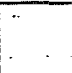 & 114 & & ${ }^{\circ} \mathrm{C} / \mathrm{W}$ & & & & \\
\hline $\begin{array}{l}\text { Case-to-Ambient } \\
\text { Thermal Registance }\end{array}$ & $\overline{\theta_{\mathrm{ca}}}$ & & 86 & $\cdots$ & ${ }^{\circ} \mathrm{C} / \mathrm{W}$ & & & & $\because$ \\
\hline
\end{tabular}


Notes:

General Note: Typical values represent the mean value of all characterization units at the nominal operating cindi-. tions. Typical drift specification are: determined by calculating the rate of change of the speeified parameter versua the drift parameter (at nominal operating conditions) for each characterization unit, and then averaging the individual unit rates. The corresponding drin figurea are normalized to the nominal oporating conditions and show how mich drift occurs as the particular drift parameter is varied from its .4. drift parameter 1s.varied from its . I! ' torr held at their nominal operating tort held at their nominal operating all characterization units as a group, as well as the \pm 2 -sigma statistical limits. Note that the typical difit specifications in the tables below may differ from the slopes of the mean curves shown in the corresponding figures.

1. HP recommends the use of noachlorine sctivated fluxes.

2. The HCPL-7800 will opereto properly at ambient temperatures up to $100^{\circ} \mathrm{C}$ but may not meet published specifications under these conditions.

3. DC performance can be best maintained by keeping $V_{D D 1}$ and $V_{D D 2}$ as close as possible to $5 \mathrm{~V}$. See application section for circuit recommendations.

4. IP recommends operation with $V_{\text {IN. }}$ $=0 \mathrm{~V}$ (tied to GND1). Limiting V V to $100 \mathrm{mV}$ will improve $\mathrm{DC}$ nonlinearity and nonlinearity drift. If $V_{\mathbb{D}}$ : is brought above $800 \mathrm{mV}$ with respect to GND1, an internal test mode may be activated. This test mode is not intended for customer

1. Although, statistically, the averrage difference in the output resistance of pins 6 and 7 is near zero, the tandard deviation of the difference in $1.3 \Omega$ due to normal process variations. Consequently, keeping the output current below $1 \mathrm{~mA}$ will onsure the best offset performance.

6. Data sheet value is the average change in offeet voltage versus temperature at $\mathrm{T}_{\mathrm{A}}=25^{\circ} \mathrm{C}$, with all other parametera held constant. This value is expressed as the change in offset voltage per ${ }^{\circ} \mathrm{C}$ change in temperature.

1.Data sheet value is the average magnitude of the change in offse voltage yersus temperature at
$T_{A}=25^{\circ} \mathrm{C}$, with all other parameter held constant. This value is . expressed as the change in magnitude per ${ }^{\circ} \mathrm{C}$ change in temperature.

8. Data theet yalue is the average changa in ofiget voltage versus: input supply voltage at $V_{D D 1}=5 \mathrm{~V}$, with all pther paramoters held constant. This value is expressed as the change in offet voltage per volt change of the input supply woltage.

9. Data gheet value is the average change in off et voltage versus output aupply voltage at $V_{\mathrm{Dp} 2}=5 \mathrm{~V}$, constant. This value is oxpressed as the change in offet. roltage per volt change of the output supply voltage.

10. Gain is defined as the slope of the beat-fit line of differential output voltage $\left(\mathrm{V}_{\mathrm{OUT}+}-\mathrm{V}_{\mathrm{OUT}}\right)$ versus differential.input yoltage $\left(V_{\mathrm{IN}}+\mathrm{Y}_{\mathrm{DV}}\right)$ over the specified input range.

11. Data gheet palue is tho average change in gain versus temperature at $\mathrm{T}_{\mathrm{A}}=25^{\circ} \mathrm{C}$, with all other parame ters held constant. This value. is . expressed as the percentage change in gain per ${ }^{\circ} \mathrm{C}$ change in temperature.

12. Data sheet value is the average magnitude of the change in gain versus temperature at $\mathrm{T}_{\mathrm{A}}=25^{\circ} \mathrm{C}$, with all other parameters hald constant. This value is expressed as constant. This value is expressed as per ${ }^{\circ} \mathrm{C}$ change in temperature.

13. Data sheet value is the average change in gain versus input supply voltage at $V_{D D 1}=5 \mathrm{~V}$, with all other parameters held constant: This value is expressed an the percentage change in gain per volt chinge of the input supply voltage.

14. Data sheet value is the sverage change in gain versus output supply voltage at $V_{D D 2}=5 \mathrm{~V}$, with all other parameters held constant: This

value is expressed as the percentage change in gajn per volt change of the output supply voltage.

15. Nonlinearity is defined as the maxi. mum deviation of the oit put voltage from the best-fit gain llne (see Note 10), expressed as a percentage of the full-scale differential output voltage range. For example, an input range of $\pm 200 \mathrm{mV}$ generates a full-scale of $\pm 200 \mathrm{mV}$ generates a full-8cale
differentfal output range of $3.2 \mathrm{~V}$

$( \pm 1.6 \mathrm{~V})$; a maximum output $( \pm 1.6 \mathrm{~V})$; a maximum output
deviation of $6.4 \mathrm{mV}$ would therefore correspond to a nonlineparity of $0.2 \%$

16. Data sheet value is the average change in nonlinearity versus tem. perature at $T_{A}=25^{\circ} \mathrm{C}$, with all other parameters held constant. This: : value is expressed as the number of percontage points that the non-

linearity will change per ${ }^{\circ} \mathrm{C}$ change in temperature. For example, if the in temperature. For example, If the tomperature in increased from $25^{\circ} \mathrm{C}$ will decrease by 0.01 percentage:. points $\left(10^{\circ} \mathrm{C}\right.$ timea $\left.-0.001 \% \mathrm{ptz} / \mathrm{C}\right)$ from $0.2 \%$ to $0.19 \%$

17. Dats sheet value in the averago . change in nonlinearity rersus input supply voltage at $V_{D D 1}=5$. , with all other parametera held constaht This value is expressed as the number of percentage points that the nonlinearity will change per volt change of the input supply voltage:

18. Data shoet value is the average change in nonlinearity versurs output supply voltage at $V_{D D 2}=5 \mathrm{~V}$, output supply voltage at $\mathrm{V}_{\mathrm{DD} 2}=$
with all other parameter held with all other parametera held constant. This yalue is expressed as
the number of percentuge points: that the nonlinearity will change per volt change of the output supply' voltage.

19. $\mathrm{NL}_{300}$ is the nonliviearity specified over an input voltage range of $\pm 100 \mathrm{mV}$.

20. Because of the switched-capacitor nature of the input sigma-delta converter, time-averaged values are shown.

21. This parameter is defined as the ratio of the differential signal gain (signal applied differentially. between pins 2 and 3 ) to the: coirimon-mode gain (input pins tied together and the signal applied to botti in puts at the same time) expressed in $\mathrm{dB}$.

22. When the differential input signal exceeds approximately $300 \mathrm{mV}$, th outputs will limit at the typical values shown.

23. The maximum specified in put supply current occurs when the differential inpüt voltage $\left(V_{\mathrm{N}_{+}}-\mathrm{V}_{\mathrm{NN}}\right)=\mathrm{O}^{\prime} \mathrm{V}$, The input supply current decreases approximately $1.3 \mathrm{~mA}$ per $1 \mathrm{~V}$ decreabe in $V_{D D 1}$.

24. The maximum specified output su pply current occurs when the dif ferential input voltage $\left(V_{I N_{+}} \cdot V_{I N}\right)$ $=200 \mathrm{mV}$, the maximum recommended operating input voltage. However, the output supply current will continue to rise for differential input voltages up to approximately $300 \mathrm{mV}$, beyond 
which the output supply current remains constant.

25. Short circuit current is the amount of outpat current generated when elther output is ehorted to $\mathrm{V}_{\mathrm{D}}$ or ground.

26. IMR (also known as CMR or Common Mode Rejectlon) apecifies the minimum rate of rise of an the minimum rate of rise of an isolation mode noise signal at which
mall output perturbations begin to apper. These output perturbation can occur with both the rising and falling edges of the isolation-mode wave forn and many be of either. polarity. When the perturbations first appiear, they cocurr only occustonally and with relatively small peak amplitudes (typically 20 30 . $30 \mathrm{~m}$ at the output of the reorm. mended appllcation circuit). Aa th magaltude of tho isolation mode. transients increase, the regularity and amplitude of the perturbations also increase.. See applications section for more information.

27. IMRR is defined as the ratio of difrerential signal gain (signal applied difforentially between pins 2 and 3) to the isolation mode gain (input pins tied to pin 4 and the - signal applied betwieen the in put and the output of the isolation amplifier) at $60 \mathrm{~Hz}$, oxpresed in $\mathrm{dB}$

28. Output noise comed from two primary sources: chopper noise and sigma-della quantization noise: Chopper noise resuits from chopper stabilization of the output op-amps It octurs at a specific frequency (typically $200 \mathrm{kHz}$ at room temper turo), and is not attenuated by the internal output Niter. A filter circuit can be easily added to the external post-amplifier to reduce the total post aut output filtar does output fler does elminate most; but not all, of the ilgma-delta quantization noiso. The magnitude of the output quantization noise is very small at lower frequencles (below $10 \mathrm{kH} z$ ) and increases with increasing frequency, Se applications section for more information. 29. Data sheet valiue is the differential amplitude of the transient at the.

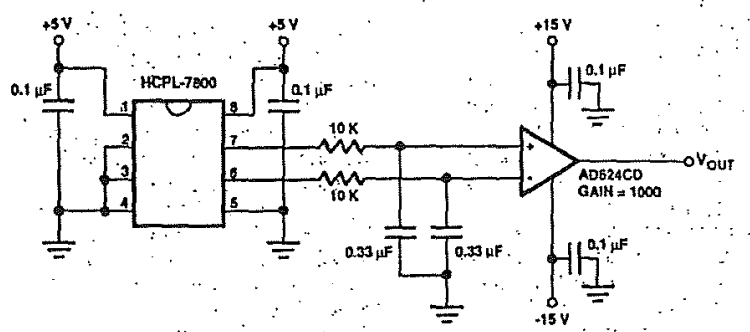

Figure 1 Input Offset Voltage Test Circuit. output of the HCPL-7800 when a $1 \mathrm{~V}_{\text {pl-pky }} 1 \mathrm{MHz}$ square wave with 5 as rise and fall times

30. This is a two pins 1-4 are shorted together and pin 60 are ahorted togother, devices with minimum $V_{\text {ISO }}$ specified at $3750 V_{\operatorname{map}}$ each optocoup pler is proof tes ted by applying an insula tion test voltage greater-than $-0 \mathrm{r}-$ equal. to $4600 \mathrm{~V}_{\mathrm{rms}}$ fot: one second (leak current detection limit, I $<6 \mu A)$. This test is performed before the method b, $100 \%$ production test for partial discharge thown in the VTF 0884 Tnulation Characteristics Table.

32. Thermal resistance values were measured on a $3^{\prime \prime} \times 3^{n}$ printed eircuit board in 'still air at room temperature with the device in a socket. ture with the device in a socket. with a thermocouple located in the center of the punderaide of the package,

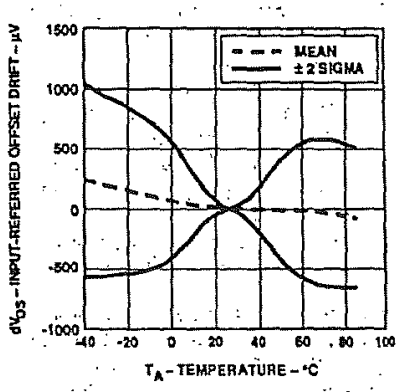

Figure 2. Input-Referred Offet. Drift va, Temperature. 

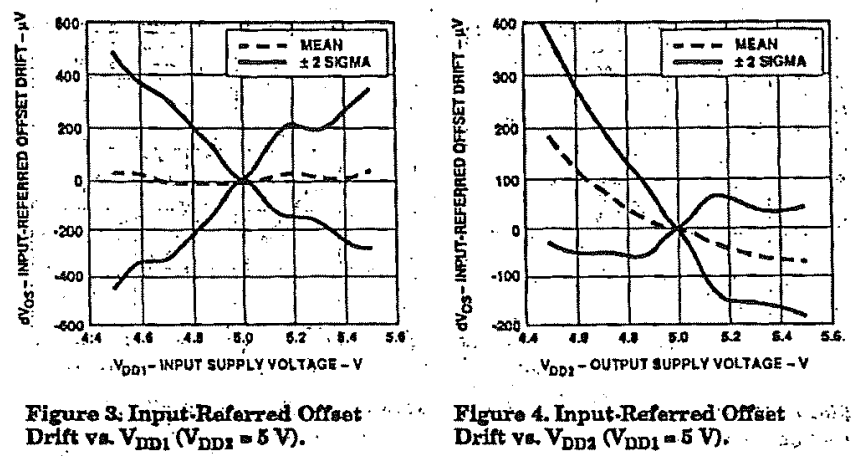

Figure 4. Input-Referred Offat $\ldots$ Drift $v a V_{D_{1}}\left(V_{D D 1}=8 \mathrm{~V}\right)$. Drift Va. $V_{D D 2}\left(V_{D D 1}=5 \mathrm{~V}\right)$.

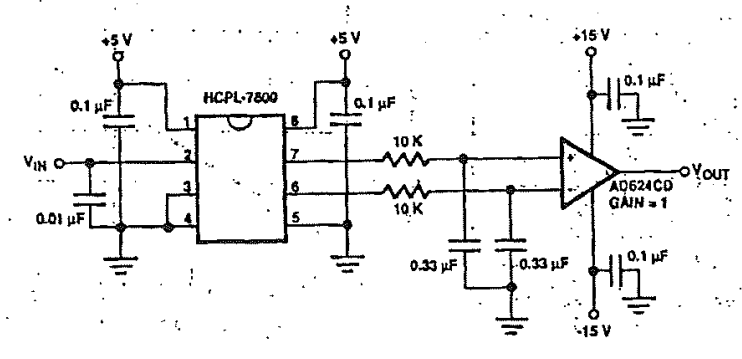

Figure E, Gain ana Nònligioarity Test Ciroult.

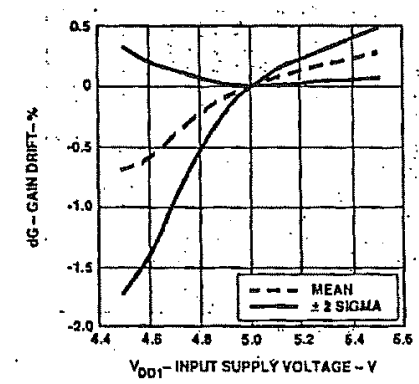

Figure 7. Gain Drift va $V_{D D 1}\left(V_{D D g}=5 V\right)$ :

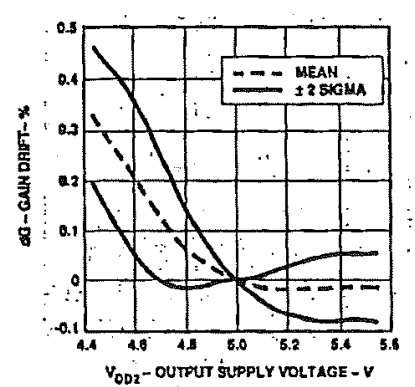

Figura 8. Gain Drift vit. $V_{p D 1}\left(V_{D D 1}=5 V\right.$.

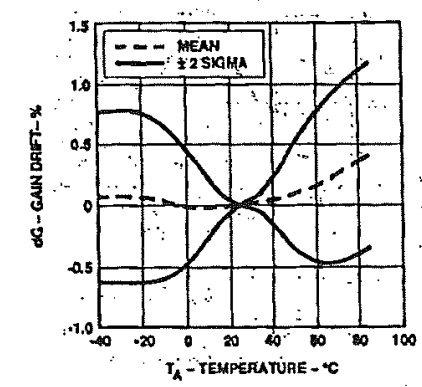

Figure 6. Gain Drift ve Temperature.

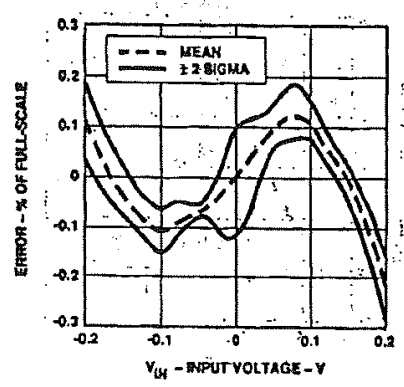

Figure 9. $200 \mathrm{mV}$ Nonlinekrity Feror Plot. 


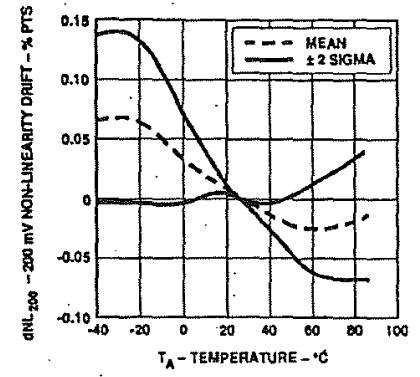

Figure 10, $200 \mathrm{mV}$ Nonlinearity Drift vs. Temperature.

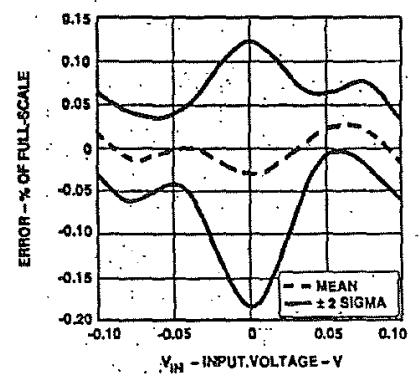

Figure 13.100 mX Nonlinearity Exrox Plot.

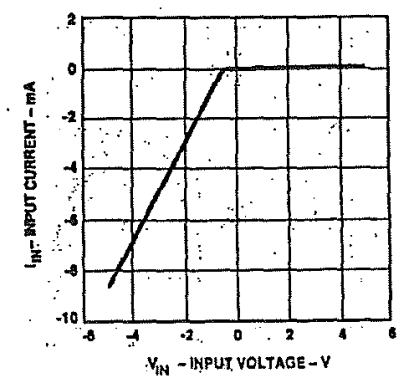

Figure 16. Typical Input Cirrent vs: Input Voltage.

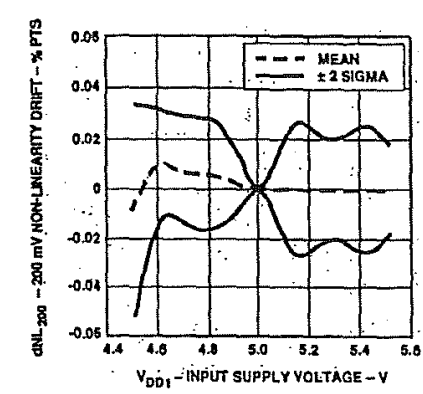

Figure 11, $200^{\circ} \mathrm{mV}$ Nonlinearity Drift จa. $V_{\text {popi }}\left(V_{\text {pDi }}\right.$ a 5 V).

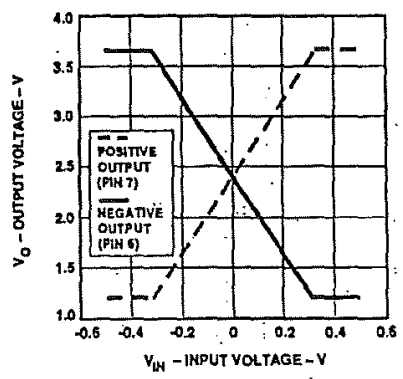

FIgure 14. Typical Oatput Voltages ve. Input Voltage.

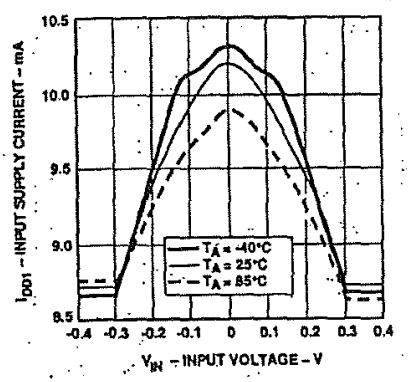

Figure 17. Typical Input Supply Current va. Input Voltage.

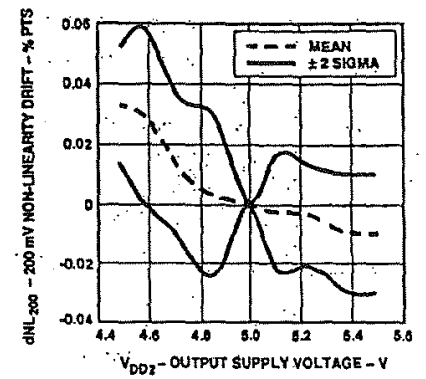

Figure 12. $200 \mathrm{mV}$ Nonlinearity Drift $\nabla=V_{D D k}\left(V_{D D 1}=5 V\right.$.

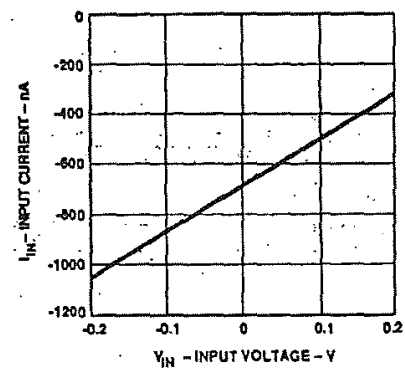

Figure 16. Typical Tnput Current vw. Input Voltage.

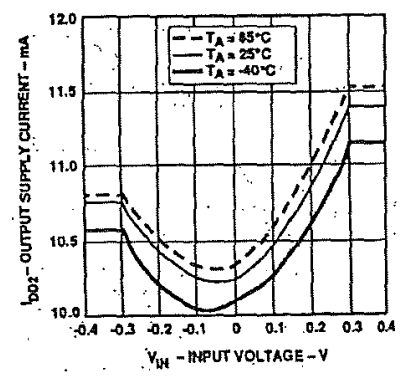

Figure 18. Typleal Output Supply Current vo. Input Voltage. 


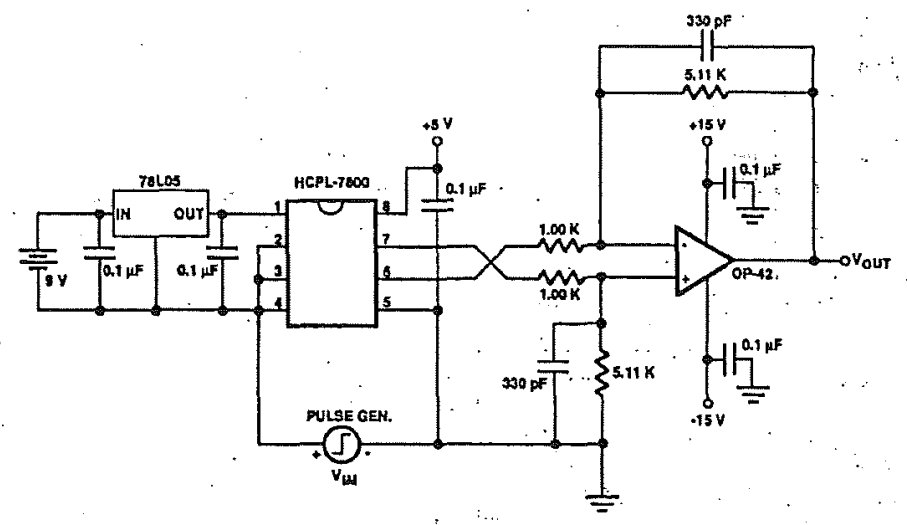

Figure 19. Isolation Modo Rejection Test Ciroult.

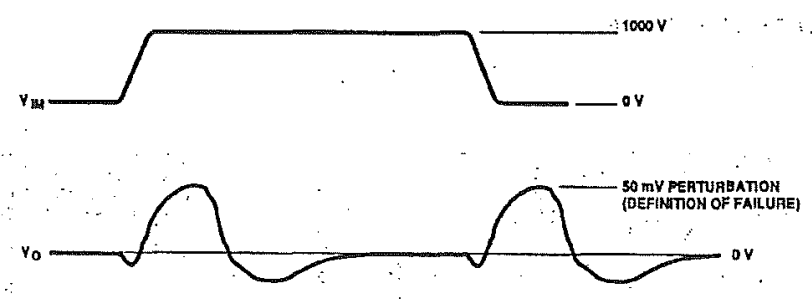

Figure 20. Typical IMR Failure Waveform.

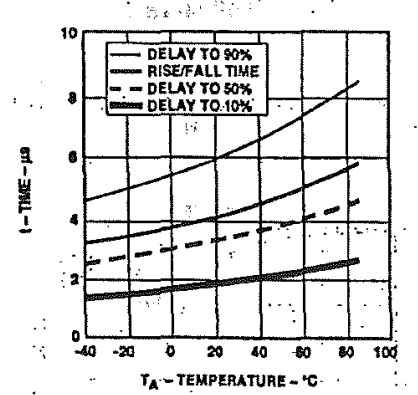

Figure 21. Typical Propagation Delays and $\mathrm{R}$ iserFall Time ve. Temperature. 

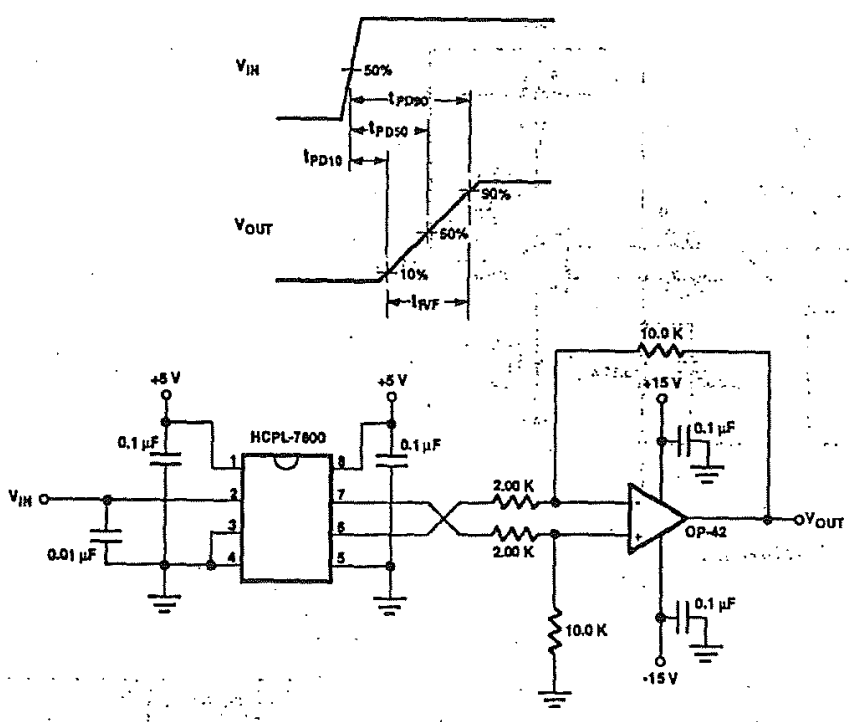

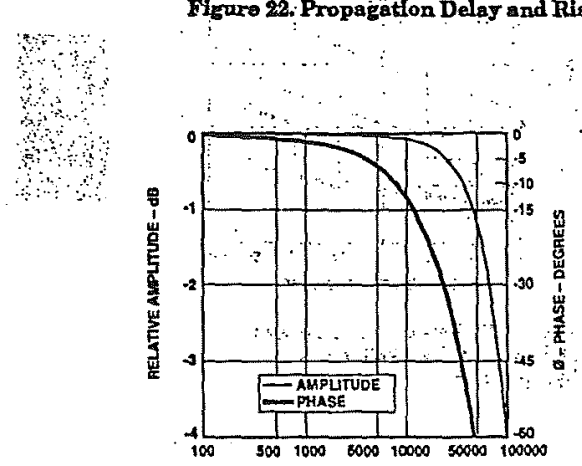

T-FAEOUENCY - Hz

Figure 23. Typlesel Amplitudo and Phase Reaponee va. Frequency.

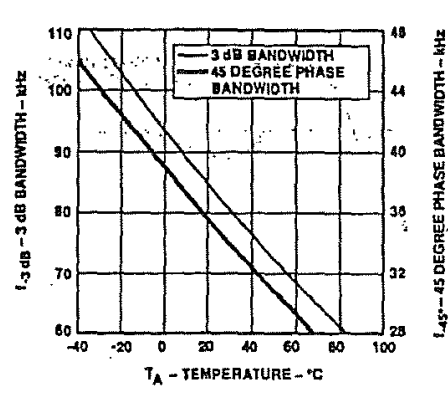

Figure 24 Typical $3 \mathrm{~dB}$ and $45^{\circ}$ Bandwidths Ys. Temperature.

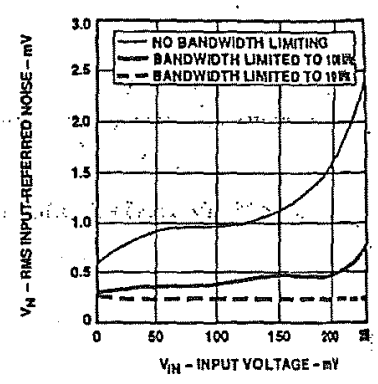

Figure 25. TYpical RMS Input. Referred Noiso ve. Input Voltage. 

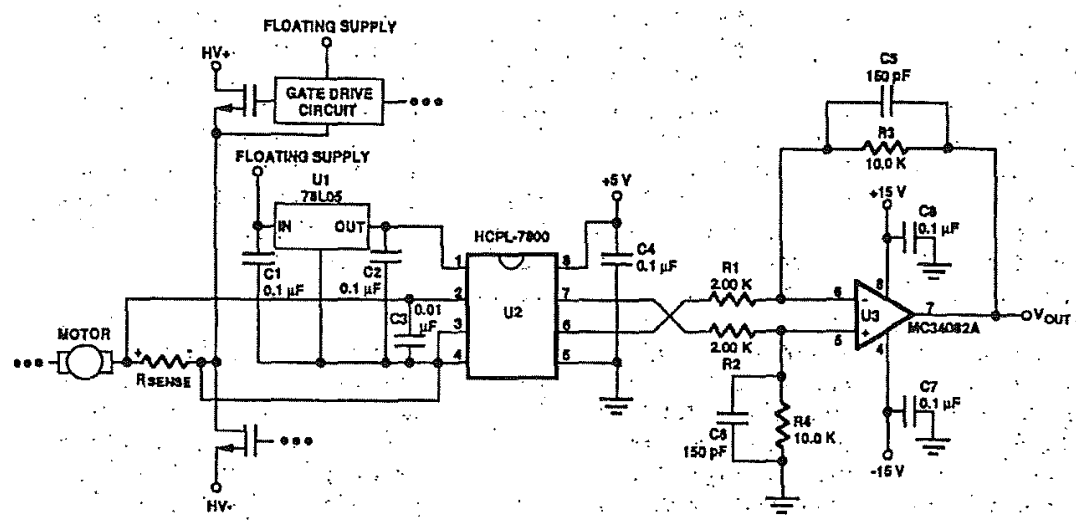

Figure 26. Recommended Application Circuit.

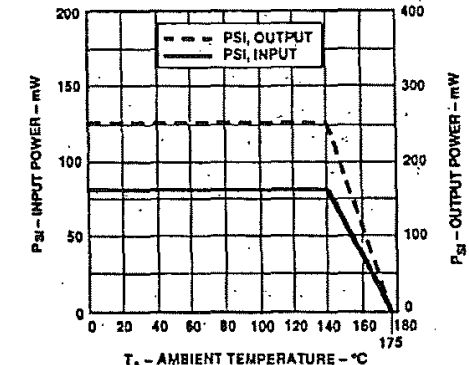

Flgare 27. Dependence of SafetyLimiting Parametera on Ambient Tampárature.

\section{Applications \\ Information \\ Functional Description \\ Figure 28 shows the primary \\ functional blocks of the \\ HCPL-7800. In operation, the \\ sigma-delta analog-to-digital \\ converter converts the analog \\ input signal into a high-speed \\ serial bit stream, the time \\ average of which is directly. \\ proportional to the input signal \\ This high speed stream of \\ digital data is encoded and}

optically transmitted to the detector circuit. The detected signal is decoded and converted into accurate analog voltage levels, which are then filtered to produce the final output signal.

To help maintain device accuracy over time and temperature, internal amplifiers are chopper-stabilized. Additionally, the encoder circuit eliminates the effects of pulse-width distortion of the optically transmitted data by generating one pulse for every edge (both rising and falling) of the converter data to be transmitted, essentially converting the widths of the sigma-delta output pulses into the positions of the encoder output pulses. A significant benefit of this coding scheme is that any non-ideal characteristics of the LED (such as nonlinearity and drift over time and temperature) have little, if any, effect on the performance of the HCPL 7800 . 
Circuit Information

The recommended application circuit is shown in Figure 26. A floating power supply (which in many applications could be the same supply that is used to drive the high-side power transistor) is regulated to $5 \cdot V$ using a simple three-terminal voltage regulator. The input of the HCPL-7800 is connected : directly to the current sensing resistor. The differential output of the isolation amplifier is converted to a groundreferenced single-ended output voltage with a simple differential amplifier circuit. Although the application circuit is relatively simple, a few general recommendations should be followed to ensure optimal performance.

As shown in Figure 26, $0.1 \mu \mathrm{F}$ bypass capacitors should be located as close as possible to the input and output power supply pins of the HCPL-7800. Notice that pin $2\left(\mathrm{~V}_{\mathrm{LN}_{+}}\right)$is bypassed with a $0.01 \mu \mathrm{F}$ capacitor to reduce input offset voltage that can be caused by the combination of long input leads and the switched-capacitor nature of the input circuit.

With pin $3\left(V_{\mathbb{N}}\right)$ tied directly to pin 4 (GND1), the power-supply. return line also functions as the sense line for the negative side of the current-sensing resistor; this allows a single twisted pair of wire to connect the isolation amplifier to the sense resistor: In some applications, however; better performance may be obtained by connecting pins 2 and $3\left(V_{\text {IN+ }}\right.$ and $\left.V_{D+}\right)$ directly: across the sense resistor with twisted pair wire and using a separate wire for the power supply return line. Both input pins should be bypassed with $0.01 \mu \mathrm{F}$ capacitors close to the isolation amplifier. In either case, it is recommended that twisted-pair wire be used to connect the isolation amplifier to the current-sensing resistor. to minimize electro-magnetic interference of the sense signal.

To obtain optimal CMR performance, the layout of the printed circuit board (PCB) should minimize any stray coupling by maintaining the maximum possible distance between the input and output sides of the circuit and ensuring that any. ground plane on the PCB does not pass directly below the HCPL-7800. An example singlesided PCB layout for the recommended application circuit is shown in Figure 29. The trace pattern is shown in "X-ray" view as it would be seen from the top of the PCB; a mirror image of this layout can be used to generate a PCB.

An inexpensive 78L05 threeterminal regulator is shown in the recommended application circuit. Because the performance of the isolation amplifier can be affected by changes in the power supply voltages, using regulators with tighter output voltage tolerances will : result in better overall circuit : performance. Many different. . regulators that provide tighter. output voltage tolerances than:.: the $78 L 05$ can be used; ; including: TL780-05 (Texas Instruments); LM340LAZ-5.0 and-LP2950CZ-5:0 (National Semiconductor):

The op-amp used in the external post-amplifier circuit should be of sufficiently high precision so that it does not contribute a significant amount of offset or offset drift relative to the contribution from the isolation amplifier. Generally, op-amps with bipolar in put stages exhibit better offset performance than op-amps with JFET or MOSFET input stages

In addition, the op-amp should also have enough bandwidth and slew rate so that it does not adversely affect the response speed of the overall circuit. The post-amplifier circuit includes a pair of capacitors ( $\mathrm{C} 5$ and $\mathrm{C6}$ ) that form a single-pole low-pass $\therefore$ afilter; these capacitors allow the bandwidth of the post-amp to be adjusted independently of the gain and are useful for reducing the output roise from the isolation amplifier. Many different op-amps could be used in the circuit, including: MC34082A (Motorola); TL032A, TLO52A, and TLC277 (Texas

Instruments); LF412A (National Semiçonductor).

The gain-setting resistors in the post-amp should have a tolerance of $1 \%$ or.better to ensure adequate CNRR and adequate gain tolerance for the overall : circuit. Resistor networks can.. be used that have much better ratio tolerances than can be achieved using discrete resis : tors. A resistor network also reduces the total number of ... components for the circuit as well as the required board space:

$\therefore \quad \therefore$ a The.current sensing resistor should have a relatively low. value of fesistance to minimize power dissipation, a fairly low inductance to aceurately reflect. high-frequency signal compo- 
nents, and a reasonably tight tolerance to maintain overall circuit accuracy. Although decreasing the value of the sense resistor decreases power dissipation, it also decreases the full-scale input voltage making iso-amp offset voltage effects more significant. These two conflicting considerations, therefore, must be weighed against each other in selecting an appropriate sense resistor for a particular application. To maintain circuit accuracy, it is recommended that the sense resistor and the isolation amplifier circuit be located as close as possible to one another. Although it is possible to buy current-sensing resistors from established vendors (e.g., the LVR-1, -3 and -5 resistors from Dale), it is also possible to make a sense resistor using a short piece of wirë or even a trace on a PC board.

Figures 30 and 31 illustrate the response of the overall isolation amplifier circuit shown in

Figure 26. Figure 30 shows the response of the circiuit to a \pm 200 . $m V 20 \mathrm{kHz}$ sine wave input and Figure 31 the response of the circuit to a $\pm 200 \mathrm{mV} 20 \mathrm{kHz}$ square wave input. Both figures demonstrate the fast, wellbehaved response of the HCPL 7800 .

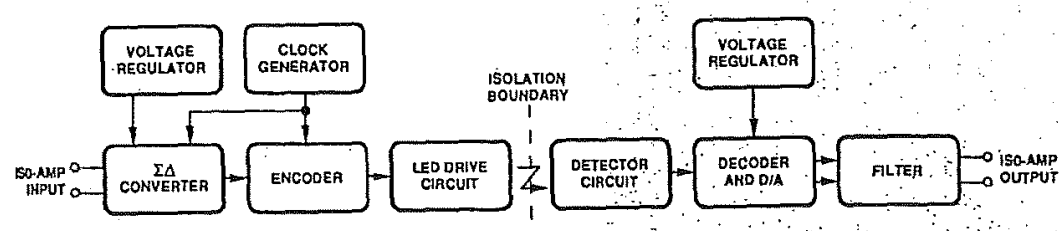

Figure 32 shows how quickly the isolation amplifier recovers from an overdrive condition generated by a $2 . \mathrm{kHz}$ square wave swinging between 0 and $500 \mathrm{mV}$ (note that the time scale is different from the previous figures). The firgt wave forin is the output of the application circuit with the filtor capacitors removed to show the actual response of the isolation amplifier. The second wave form is the response of the same circuit with the capacitors installed. The recovery time and overshoot are relatively independent of the amplitude and polarity of the overdrive signal, as well as its duration:

For more information, refer to Application Note 1059.

Figure 28. HCPL-7800 Block Diagram.
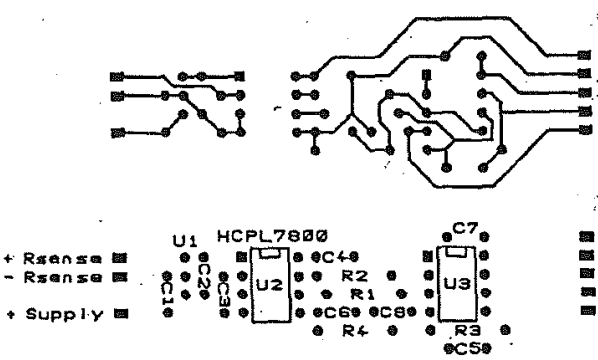

a volts

inound

mour vol.rs

Flgure 29. PC Board Trace Pattern and Loading Diagram Ecample. 


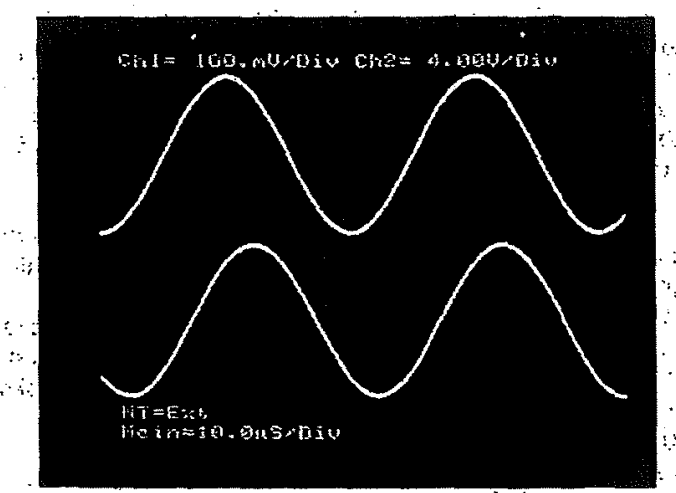

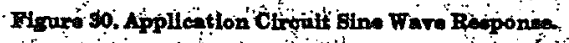

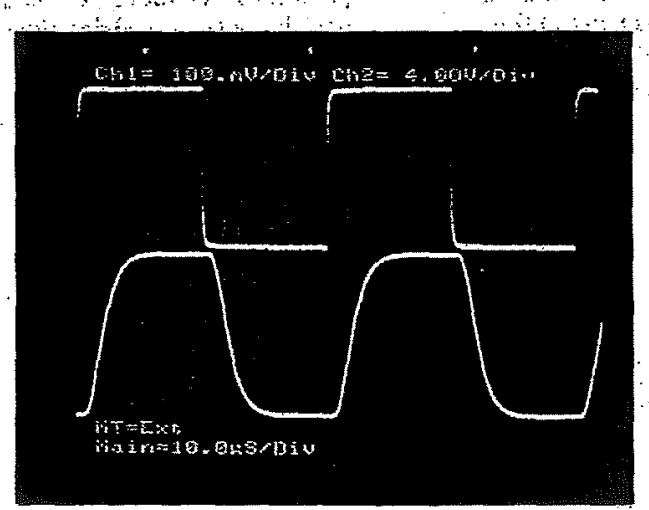

Figure 31. Application Ciroult Bquare Wave Responee.

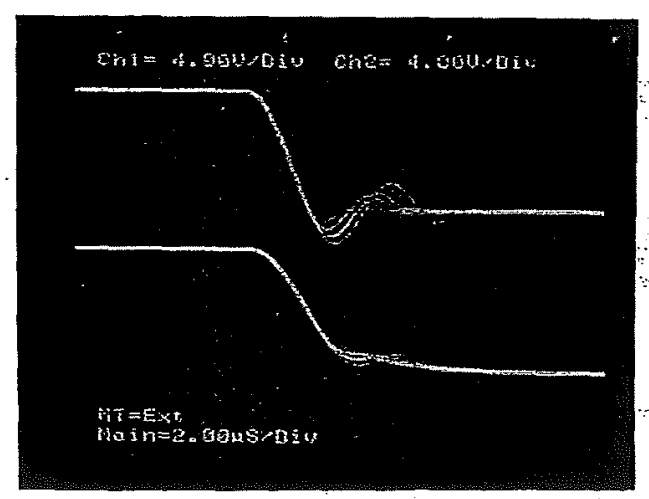

Figure 32. Application Circult Ovorload Recovery Wavatorm.

6-236 


\section{D4. MAX7219 LED Display Driver}

19-4452: Rev 2: 11,96

\section{MUXIMI}

Serially Interfaced, 8-Digit LED Display Driver

\section{General Description}

The MAX7219 is a compact, serial input/output common cathode display driver that interfaces microprocessors (uPs) to 7-segment numeric LED displays of up to 8 digits, bar-graph displays, or 64 individual LEDs. Included on-chip are a BCD code-B decoder, multiplex scan circuitry, segment and digit drivers, and an $8 \times 8$ static RAM that stores each digit. Only one external resistor is required to set the segment current for all LEDs.

A convenient 3-wire serial interface connects to all common $\mu$ Ps. Individual digits may be addressed and up dated without rewriting the entire display. The MAX7219 also allows the user to select code-B decoding or no decode for each digit.

The MAX7219 includes a 150 A low-power shutdown mode, analog and digital brightness control, a scan-limit register which allows the user to display from 1 to 8 digits, and a test mode which forces all LEDs on.

Bar-Graph Displays

7-Segment Displays

Industrial Controllers

Panel Meters

LED Matrix Displays

Pin Configuration

TOP VIEW

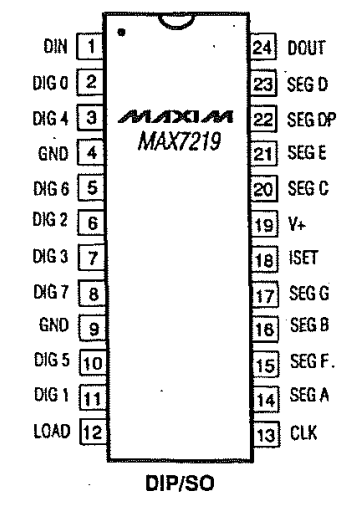

Applications
10MHz Serial Interface

Individual LED Segment Control

- Decode/No-Decode Digit Selection

- 150 $\mu$ A Low-Power Shutdown (Data Retalned)

- Digital and Analog Brightness Control

- Display Blanked on Power-Up

24-PIn DIP and SO Packages

- Drives Common-Cathode LED Display

Ordering Information

\begin{tabular}{|lll|}
\hline PART & TEMP. RANGE & \multicolumn{1}{c|}{ PIN-PACKAGE } \\
\hline MAX7219CNG & $0^{\circ} \mathrm{C}$ to $+70^{\circ} \mathrm{C}$ & 24 Narrow Plastic DIP \\
\hline MAX7219CWG & $0^{\circ} \mathrm{C}$ to $+70^{\circ} \mathrm{C}$ & 24 Wide SO \\
\hline MAX7219C/O & $0^{\circ} \mathrm{C}$ to $+70^{\circ} \mathrm{C}$ & Dice \\
\hline MAX7219ENG & $-40^{\circ} \mathrm{C}$ to $+85^{\circ} \mathrm{C}$ & 24 Narrow Plastic DIP \\
\hline MAX7219EWG & $-40^{\circ} \mathrm{C}$ to $+85^{\circ} \mathrm{C}$ & 24 Wide SO \\
\hline MAX7219EAG & $-40^{\circ} \mathrm{C}$ to $+85^{\circ} \mathrm{C}$ & 24 Narrow CERDIP \\
\hline
\end{tabular}

- Contact factory for dice specilications.

Typical Application Circuit

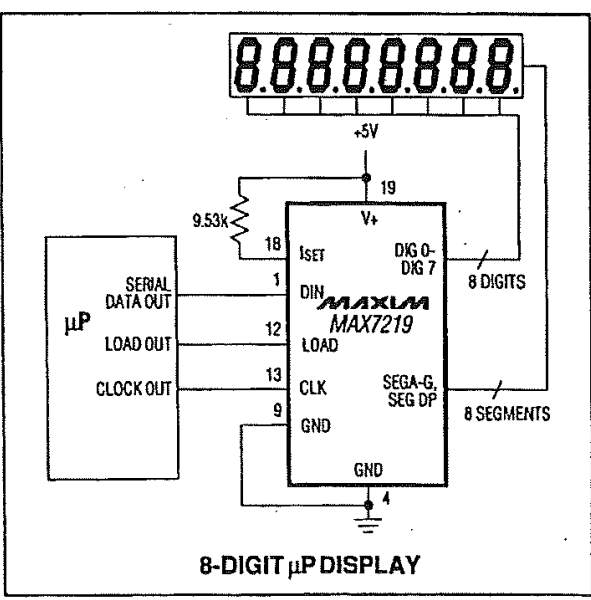

Maxim Integrated Products

MAXIM

For free samples \& the latest literature: http://www.maxim-ic.com, or phone 1-800-998-8800 


\section{Serially Interfaced, 8-Digit LED Display Driver}

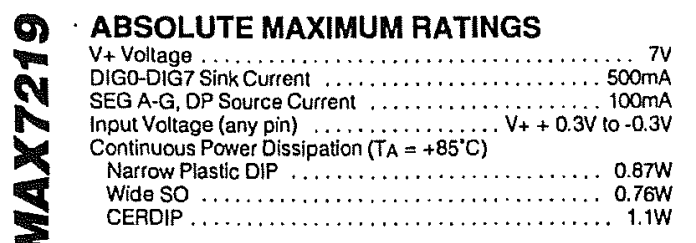

Operating Temperature Ranges:

MAX7219C G $G \ldots \ldots \ldots \ldots \ldots \ldots, 0^{\circ} \mathrm{C}$ to $+70^{\circ} \mathrm{C}$ MAX7219E_ $G \ldots \ldots \ldots \ldots \ldots \ldots \ldots \ldots \ldots, 40^{\circ} \mathrm{C}$ to $+85^{\circ} \mathrm{C}$ Storage Temperature Range ................ $-65^{\circ} \mathrm{C}$ to $+160^{\circ} \mathrm{C}$ Lead Temperalure (soldering, $10 \mathrm{sec}) \ldots \ldots \ldots \ldots \ldots+300^{\circ} \mathrm{C}$

Wide SO

Stresses beyond those listed under "Absolute Maximum Batings" may cause permanent demage to the device. These are stress ratings only. and functional operals bexpesure to

ELECTRICAL CHARACTERISTICS

$\left(V+=5 V \pm 10 \%\right.$, RSET $=9.53 k \Omega \pm 1 \% . T_{A}=T_{M I N}$ to $T_{M A X}$, unless otherwise noted. $)$

\begin{tabular}{|c|c|c|c|c|c|c|}
\hline PARAMETER & SYMBOL & CONDITIONS & MiN & TYP & MAX & UNITS \\
\hline Operating Supply Voltage & $v_{+}$ & & 4.0 & & 6.0 & v \\
\hline Shutdown Supply Current & 10 & $\begin{array}{l}\text { DIN,CLK and } L O A D=G N D \text { or } V_{+} \\
\text {shutdown register sel to } 0, T_{A}=+25^{\circ} \mathrm{C}\end{array}$ & & & 150 & $\mu \mathrm{A}$ \\
\hline \multirow[b]{2}{*}{ Operating Supply Current } & \multirow[b]{2}{*}{ lop } & RSET $=$ infinity & & & 8 & $\mathrm{~mA}$ \\
\hline & & $\begin{array}{l}\text { All segments and decimal points on, } \\
\text { ISEGO }=-40 \mathrm{~mA}\end{array}$ & \multicolumn{3}{|c|}{330} & $\mathrm{~mA}$ \\
\hline Display Scan Rate & $\operatorname{lose}$ & $V_{+}=5 \mathrm{~V}, 8$ digits scanned & 500 & 1300 & 2000 & $\mathrm{~Hz}$ \\
\hline Digit Drive Sink Current & IDIGI & $T_{A}=+25^{\circ} \mathrm{C}, V_{+}=5 \mathrm{~V}, V_{\text {OUT }}=0.65 \mathrm{~V}$ & 320 & & & $\mathrm{~mA}$ \\
\hline Segment Drive Current Source & ISEGO & $T_{A}=+25^{\circ} \mathrm{C}, V_{+}=5 \mathrm{~V}, V_{\text {OUT }}=V_{+}-1 \mathrm{~V}$ & -30 & -37 & -40 & mA \\
\hline Segment Drive Current Matching & & 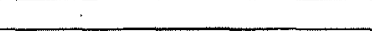 & & $3.0^{\circ}$ & & $\%$ \\
\hline Digit Drive Source Current & loigo & Digit off, VOUT $=V+-0.3 \mathrm{~V}$ & -2 & & & $\mathrm{~mA}$ \\
\hline Segment Drive Current Sink & ISEGI & Segment ofl. $V_{\text {OUT }}=0.3 \mathrm{~V}$ & 5 & & & $\mathrm{~mA}$ \\
\hline \multicolumn{7}{|l|}{ LOGIC INPUTS } \\
\hline Input Current & 1 & $\begin{array}{l}\text { DIN. CLK and LOAD } \\
V_{I N}=O V \\
V_{I N}=V_{+}\end{array}$ & & & -1 & $\mu A$ \\
\hline Logic 1 Inpul Voltage & $V_{\mathrm{HH}}$ & & 3.5 & & & $\mathrm{v}$ \\
\hline Logic o Input Voltage & $V_{I L}$ & & & & 0.8 & $\mathrm{~V}$ \\
\hline Hysteresis Voltage & & DIN, CLK, and LOAD & & 1.0 & & v \\
\hline Outpul High Voltage & $\mathrm{V}_{\mathrm{OH}}$ & $\begin{array}{l}\text { DOUT } \\
\text { lOUT }=-1 \mathrm{~mA} \text {, } \\
\text { lOUT }-1 \mu \mathrm{A}\end{array}$ & $v_{+-1.0}$ & $V_{+}$ & & v \\
\hline Output Low Voltage & $\mathrm{V}_{\mathrm{OL}}$ & DOUT, IOUT $=1.6 \mathrm{~mA}$ & & & 0.4 & $\mathrm{v}$ \\
\hline Data-Hold Time DATAIN to Clock & $\mathrm{EDH}$ & & 0 & .5 & & ns \\
\hline Data-Setup Time DATAIN To Clock & tos & & 25 & & & ns \\
\hline Clock-1o-Serial Outpul Prop Delay & topD & $C_{L C A D}=50 p F$ & & & 25 & ns \\
\hline Clock Low Tine & tCKL & & 50 & & & ns \\
\hline Clock High Time & LCKH & & 50 & & & ns \\
\hline Data-to Segment Prop Delay (Note 1) & LOSPD & $C_{Z Q A D}=50 \rho \mathrm{F}$ & 0 & & 2.25 & $\mathrm{~ms}$ \\
\hline $\begin{array}{l}\text { Load-Rising Edge to Nexl Clock } \\
\text { Aising Edge }\end{array}$ & LLOCK & & 50 & & & ns \\
\hline $\begin{array}{l}\text { Clock-to-Load Rising Edge } \\
\text { Setup Time }\end{array}$ & ICKLD & & 0 & & & ns \\
\hline Load Low Time & LLOL & & 50 & & & $\mathrm{~ns}$ \\
\hline Load High Time & $\mathrm{LOH}$ & & 50 & & & ns \\
\hline
\end{tabular}

Note 1: Guaranteed by design. 


\section{Serially Interfaced, 8-Digit LED Display Driver}

Typical Operating Characteristics
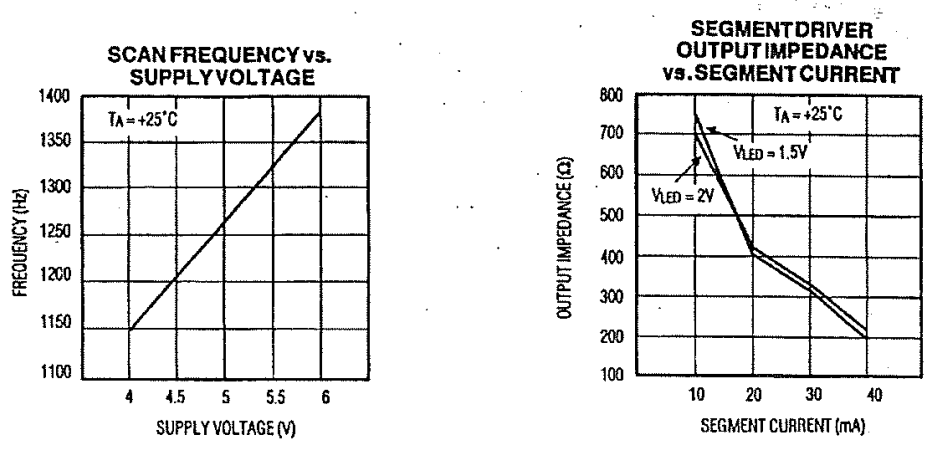

Pin Description

\begin{tabular}{|c|c|c|}
\hline PIN & NAME & FUNCTION \\
\hline 1 & DIN & Serial Data Inpul. Data is loaded into an internal 16-bit shift register on the rising edge of $\mathrm{CLK}$. \\
\hline $2,3,5-8,10,11$ & DIG0-7 & 8 digit drive lines that sink current from the display. \\
\hline 4,9 & GND & Ground (both GND pins must be connected) \\
\hline 12 & LOAD & Load Data Input. On LOAD's rising edge, the last 16 bits of serial inpul data are latched. \\
\hline 13 & CLK & $\begin{array}{l}\text { Clock Input. 10MHiz maximum rale. On CLK's rising edge, data is shitted into the internal shift } \\
\text { register. On CLK' falling edge, data is clocked out of DOUU?. }\end{array}$ \\
\hline $14-17,20-23$ & SEG A-G,DP & 7-segment drive and decimal point lines that scurce current to the display. \\
\hline 18 & ISET & $\begin{array}{l}\text { Connect to } V+\text { through a resistor (RSET) to set the peak segment current } \\
\text { (Reler to "Selecting RSET Resistor section). }\end{array}$ \\
\hline 19 & $v_{+}$ & Supply Vollaga \\
\hline 24 & DOUT & Serial Data Output. The data into DIN is valid at DOUT 16.5 clock cycles later. \\
\hline
\end{tabular}


Serially Interfaced, 8-Digit LED Display Driver

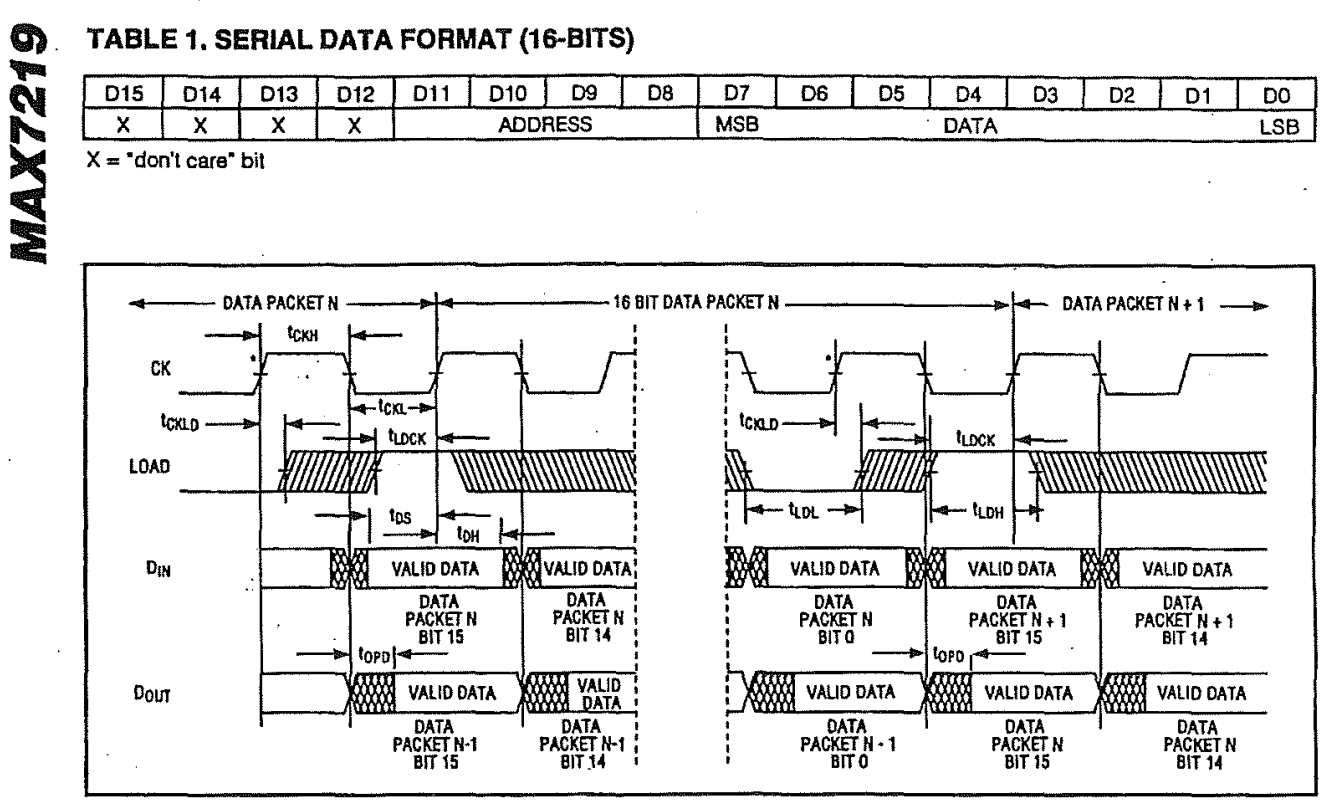

Figure 1a. Timing Diagram

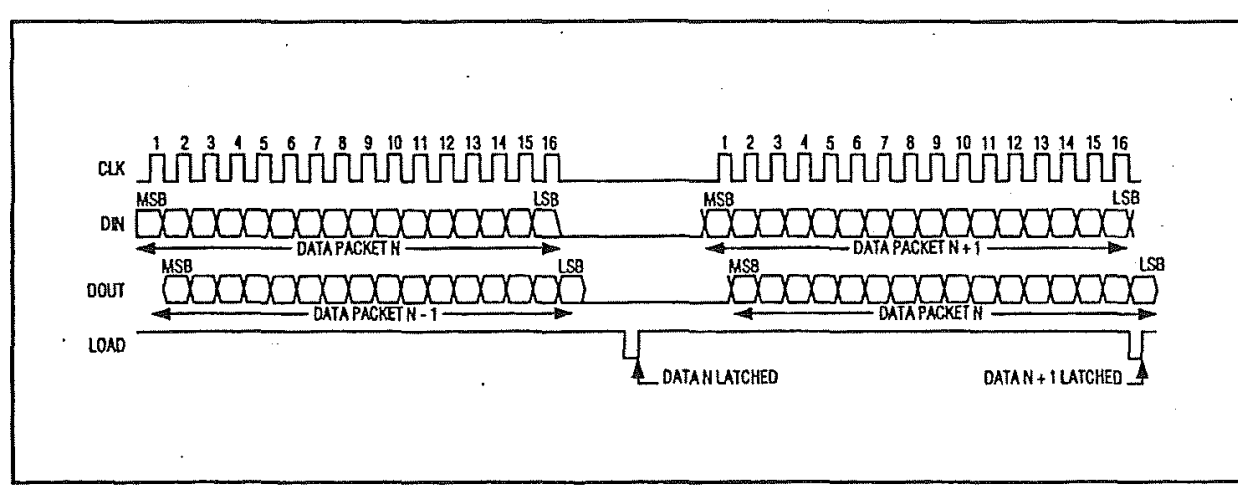

Figura 1b. Timing Diagram 


\section{Serially Interfaced, 8-Digit LED Display Driver}

\section{Detailed Description \\ Serial Addressing Modes} Serial data at DIN, sent in 16-bit packets, is shifted into the internal 16-bit shift register with each rising edge of CLK (Figures $1 a$ and $1 b$ ). The data is then latched into either the digit or control reglsters on the rising edge of LOAD. LOAD must go high concurrently with or aftar the 16th rising clock edge, but before the next rising clock edge or data will be lost. Data at DIN is propagated through the shift register and appears at DOUT 16.5 clock cycles later. Data is clocked out on the falling edge of CLK. Data bits are labeled DO-D15 (Table 1). D8-D11 contain the register address, D0-D7 contain the data, and D12-D15 are "don't care" bits. The first bit received is D15, the most significant bit (MSB)

Digit and Control Registers

Table 2 lists the 14 addressable digit and control registers. The digit registers are realized with an on-chip, $8 \times 8$ dual-port SRAM. They are addressed directly so that individual digits can be updated and retain data as long as V+typically exceeds $2 \mathrm{~V}$. The control registers consis of: decode mode, display intensity, scan limit (number of of: decode mode, display intensity, scan limit (number of A no-operation (no-op) register is also included, which allows data to be passed from DIN to DOUT when devices are cascaded without changing the display or affecting any control registers.

Shutdown Mode

When the MAX7219 is in shutdown mode, the scan oscillator is halted, all segment current sources are pulled to ground, and all digit drivers are pulled to $\mathrm{V}_{+}$, thereby blanking the display. Data in the digit and control registers remains unaltered. Shutdown can be used to save power or as an alarm to flash the display by successively entering and leaving the shutdown mode. For minimum supply current in shutdown mode, logic inputs should be at ground or V+ (CMOS logic levels).

Typically, it takes less than 250 us for the MAX7219 to leave shutdown mode. Note that the display driver can still be programmed while in shutdown mode, and that shutdow mode can be overridden by the display-lest function.

Table 4. Decode-Mode Register Examples (Address (Hex) $=X 9$ )

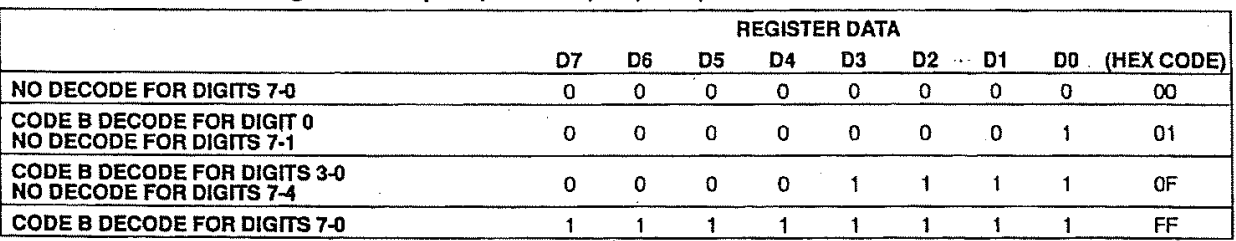

Table 2. Register Address Map
\begin{tabular}{|lcccccc|}
\hline REGISTER & \multicolumn{7}{c|}{ ADDRESS } \\
\hline & D15-D12 & D11 & D10 & D9 & D8 & CODE \\
\hline NO-OP & $X$ & 0 & 0 & 0 & 0 & $X 0$ \\
\hline DIGTT O & $X$ & 0 & 0 & 0 & 1 & $X 1$ \\
\hline DIGIT 1 & $X$ & 0 & 0 & 1 & 0 & $X 2$ \\
\hline DIGIT 2 & $X$ & 0 & 0 & 1 & 1 & $X 3$ \\
\hline DIGT 3 & $X$ & 0 & 1 & 0 & 0 & $X 4$ \\
\hline DIGIT 4 & $X$ & 0 & 1 & 0 & 1 & $X 5$ \\
\hline DIGIT 5 & $X$ & 0 & 1 & 1 & 0 & $X 6$ \\
\hline DIGIT 6 & $X$ & 0 & 1 & 1 & 1 & $X 7$ \\
\hline DIGIT 7 & $X$ & 1 & 0 & 0 & 0 & $X 8$ \\
\hline DECODE MODE & $X$ & 1 & 0 & 0 & 1 & $X 9$ \\
\hline INTENSITY & $X$ & 1 & 0 & 1 & 0 & $X A$ \\
\hline SCAN LIMIT & $X$ & 1 & 0 & 1 & 1 & $X 8$ \\
\hline SHUTDOWN & $X$ & 1 & 1 & 0 & 0 & $X C$ \\
\hline DISPLAY TEST & $X$ & 1 & 1 & 1 & 1 & $X F$ \\
\hline
\end{tabular}

Table 3. Shutdown Register Format

\begin{tabular}{|c|c|c|}
\hline & $\begin{array}{l}\text { ADDR CODE } \\
\text { (HEX) }\end{array}$ & $\begin{array}{c}\text { REGISTER DATA } \\
\text { D7 D6 D5 D4 D3 D2 D1 Do }\end{array}$ \\
\hline $\begin{array}{l}\text { SHUTDOWN } \\
\text { MODE }\end{array}$ & $x c$ & $x \times x \times x \times x \times 0$ \\
\hline $\begin{array}{l}\text { NORMAL } \\
\text { OPERATION }\end{array}$ & $x C$ & $\begin{array}{lllllllll}x & \times & x & \times & \times & \times & \times & 1\end{array}$ \\
\hline
\end{tabular}

Initial Power. Up

On initial power-up, all control registers are reset, the display is blanked, and the MAX7219 enters shutdown mode. Therefore the user must program the display driver prior to display use since it will initially be set to scan one digit, it will not decode data in the data registers, and the intensity register will be set to its minimum value.

Decode-Mode Register

The decode-mode register sets $B C D$ code $B(0-9, E, H$ $L, P$, and - - or no-decode operation for each digit. Each bit in the register corresponds to one digit. A logic high selects code $B$ decoding while a logic low bypasses the decoder. Examples of the decode mode control-register format are shown in Table 4. (Address $(\mathrm{HeX})=\mathrm{XC}$ ) 
Serially Interfaced, 8-Digit LED Display Driver

N

Table 5. Code B Font

\begin{tabular}{|c|c|c|c|c|c|c|c|c|c|c|c|c|c|c|}
\hline \multirow{2}{*}{$\begin{array}{l}\text { 7-SEGMENT } \\
\text { CHARACTER }\end{array}$} & \multicolumn{6}{|c|}{ REGISTER DATA } & \multicolumn{8}{|c|}{ ON SEGMENTS $=1$} \\
\hline & D7* & D6-D4 & D3 & D2 & D1 & DO & DPA & $\mathbf{A}$ & B & c & $D$ & $\mathbf{E}$ & $\mathbf{F}$ & G \\
\hline 0 & & $x$ & 0 & 0 & 0 & 0 & & 1 & 1 & 1 & 1 & 1 & 1 & 0 \\
\hline 1 & & $x$ & 0 & 0 & 0 & 1 & & 0 & 1 & 1 & 0 & 0 & 0 & 0 \\
\hline 2 & & $x$ & 0 & 0 & 1 & 0 & & 1 & 1 & 0 & 1 & 1 & 0 & 1 \\
\hline 3 & & $x$ & 0 & 0 & 1 & 1 & & 1 & 1 & 1 & 1 & 0 & 0 & 1 \\
\hline 4 & & $x$ & 0 & 1 & 0 & 0 & & 0 & 1 & 1 & 0 & 0 & 1. & 1 \\
\hline 5 & & $x$ & 0 & 1 & 0 & 1 & & 1 & 0 & 1 & 1 & 0 & 1 & 1 \\
\hline 6 & & $x$ & 0 & 1 & 1 & 0 & & 1 & 0 & 1 & 1 & 1 & 1 & 1 \\
\hline 7 & & $x$ & 0 & 1 & 1 & 1 & & 1 & 1 & 1 & 0 & 0 & 0 & 0 \\
\hline 8 & & $x$ & 1 & 0 & 0 & 0 & & 1 & 1 & 1 & 1 & 1 & 1 & 1 \\
\hline 9 & & $x$ & 1 & 0 & 0 & 1 & & 1 & 1 & 1 & 1 & 0 & 1 & 1 \\
\hline- & & $x$ & 1 & 0 & 1 & 0 & & 0 & 0 & 0 & 0 & 0 & 0 & 1 \\
\hline$E$ & & $x$ & 1 & 0 & 1 & 1 & & 1 & 0 & 0 & 1 & 1 & 1 & 1 \\
\hline $\mathrm{H}$ & & $x$ & 1 & 1 & 0 & 0 & & 0 & 1 & 1 & 0 & 1 & 1 & 1 \\
\hline$L$ & & $x$ & 1 & 1 & 0 & 1 & & 0 & 0 & 0 & 1 & 1 & 1 & 0 \\
\hline$P$ & & $x$ & 1 & 1 & 1 & 0 & & 1 & 1 & 0 & 0 & 1 & 1 & 1 \\
\hline blank & & $x$ & 1 & 1 & 1 & 1 & & 0 & 0 & 0 & 0 & 0 & 0 & 0 \\
\hline
\end{tabular}

- The decimal point is set by bil $07=1$

When the code $B$ decode mode is used, the decoder looks only at the lower nibble of the data in the digit registers (D3-DO), distegarding bits D4-D6. D7, which sets the decimal point (SEG DP), is independent of the decoder and is positive logic (D7 $7=1$ turns the decimal point on). The code-B font is listed in Table 5.

When no-decode is selected, data bits D7-DO correspond to the segment lines of the MAX7219. Table 6 shows the one-to-one pairing of each data bit to the appropriate segment line.

Table 6. No-decode Mode Data Blts and Corresponding Segment Lines

$$
\text { STANDARD 7-SEGMENTLED }
$$

\begin{tabular}{|llllllllll|}
\hline \multicolumn{1}{c|}{ AEGISTER DATA } \\
& D7 & D6 & D5 & D4 & D3 & D2 & D1 & DO \\
\hline $\begin{array}{lllllllllll}\text { CORAESPONDING } \\
\text { SEGMENT LINE }\end{array}$ & DP & A & B & C & D & E & F & $G$ \\
\hline
\end{tabular}

Intensity Control and Interdigit Blanking

The MAX7219 allows the display brightness to be controlled with an external resistor (RSET) connected between $V_{+}$and ISET, and digitally using the intensity register. The peak current sourced
from the segment drivers will nominally be 100 from the segment drivers will nominally be 100 either be fixed, or variable to allow brightness adjustment from the front panel. Its minimum value should be $9.53 \mathrm{k} \Omega$, which typically sets the segment current at $37 \mathrm{~mA}$.

Digital control of segment current is provided by an internal pulse-width modulated DAC, which: is loaded from the lower nibble of the intensity register. The DAC scales the average segment current in 16 steps from a maximum of $31 / 32$, down to $1 / 32$ of the peak current set by RSET. The intensity register format is listed in Table 7. Maximum brightness occurs with a duty cycle of $31 / 32$ because the interdigit blanking time is set to $1 / 32$ of a.cycle. Interdigit blanking time can be increased by decreasing the duty cycle. 


\section{Serially Interfaced, 8-Digit LED Display Driver}

\begin{tabular}{|c|c|c|c|c|c|c|c|c|c|}
\hline \multirow{2}{*}{$\begin{array}{l}\text { DUTY } \\
\text { CYCLE }\end{array}$} & \multicolumn{8}{|c|}{ REGISTER DATA } & \multirow{2}{*}{$\begin{array}{l}\text { (HEX } \\
\text { CODE) }\end{array}$} \\
\hline & D7 & D6 & D5 & D4 & D3 & D2 & D1 & Do & \\
\hline $\begin{array}{c}1 / 32 \\
\text { (min on) }\end{array}$ & $x$ & $x$ & $x$ & $x$ & 0 & 0 & 0 & 0 & xo \\
\hline $3 / 32$ & $x$ & $x$ & $x$ & $x$ & 0 & 0 & 0 & 1 & $x_{1}$ \\
\hline $5 / 32$ & $x$ & $x$ & $x$ & $x$ & 0 & 0 & 1 & 0 & $\times 2$ \\
\hline $7 / 32$ & $x$ & $x$ & $x$ & $x$ & 0 & 0 & 1 & 1 & $\times 3$ \\
\hline $9 / 32$ & $x$ & $x$ & $x$ & $x$ & 0 & 1 & 0 & 0 & $\times 4$ \\
\hline $11 / 32$ & $x$ & $x$ & $x$ & $x$ & 0 & 1 & 0 & 1 & $\times 5$ \\
\hline $13 / 32$ & $x$ & $x$ & $x$ & $x$ & 0 & 1 & 1 & 0 & $x_{6}$ \\
\hline $15 / 32$ & $x$ & $x$ & $x$ & $\bar{x}$ & 0 & 1 & 1 & 1 & $x 7$ \\
\hline $17 / 32$ & $x$ & $x$ & $x$ & $x$ & 1 & 0 & 0 & 0 & $x 8$ \\
\hline $19 / 32$ & $x$ & $x$ & $x$ & $x$ & 1 & 0 & 0 & 1 & $x 9$ \\
\hline $21 / 32$ & $x$ & $x$ & $x$ & $x$ & 1 & 0 & 1 & 0 & $X A$ \\
\hline $23 / 32$ & $x$ & $x$ & $x$ & $\mathrm{x}$ & 1 & 0 & 1 & 1 & $\mathrm{XB}$ \\
\hline $25 / 32$ & $x$ & $x$ & $x$ & $x$ & 1 & 1 & 0 & 0 & $x c$ \\
\hline $27 / 32$ & $x$ & $x$ & $x$ & $x$ & 1 & 1 & 0 & 1 & $X D$ \\
\hline $29 / 32$ & $x$ & $x$ & $x$ & $x$ & 1 & 1 & 1 & 0 & $X E$ \\
\hline $\begin{array}{c}31 / 32 \\
(\max \text { on })\end{array}$ & $x$ & $x$ & $x$ & $x$ & 1 & 1 & 1 & 1 & $X F$ \\
\hline
\end{tabular}

Scan-Limit Register

The scan-limit register sets how many digits are displayed from 1 to 8 . They are displayed in a multiplexed manner with a typical display scan rate of $1300 \mathrm{~Hz}$ with 8 digits displayed. If fewer digits are displayed, the scan rate is $810 s c / N$, where $N$ is the number of digits scanned. Since the number of scanned digits affects the display brightness, the scan-limit register should not be used to blank portions of the display (such as leading zero suppres sion). The scan-limit register format is listed in Table 8 . If the scan-limit register is set for three digits or less, individual digit drivers will dissipate excessive amounts of power. Consequently, the value of the RSET resistor must be adjusted according to the number of digits displayed, to limit individual digit driver power dissipation. Table 9 lists the number of digits displayed and the corresponding maximum recommended segment current when the internal digit drivers are used.

\section{Display-Test Register}

The display-test register operales in two modes: nomal and display test. Display-test mode turns all LEDs on by overriding - but not altering - all controls and digit registers (including the shutdown register). In displaytest mode, 8 digits are scanned and the duty cycle is $31 / 32$. Table 9 lists the display-test register format.
Table 8. Scan-Limit Register Format

(Address $($ Hex) $=$ XB)

\begin{tabular}{|c|c|c|c|}
\hline & $\begin{array}{c}\text { REGISTER DATA } \\
\text { D7 D6 D5 D4 D3 D2 D1 D }\end{array}$ & & $\begin{array}{l}\text { (MEX } \\
\text { CODE) }\end{array}$ \\
\hline - OISPLAY DIGIT O ONLY & $x \times \times \times \times 000$ & 0 & $x_{0}$ \\
\hline "DISPLAY DIGITS O \& 1 & $x \times \times \times \times 0001$ & 1 & $x 1$ \\
\hline -DISPLAY DIGITS 012 & $x \times \times \times \times 010$ & 0 & $x_{2}$ \\
\hline DISPLAY DIGITS 0123 & $x \times \times \times \times 011$ & 1 & $\times 3$ \\
\hline $\begin{array}{l}\text { DISPLAY DIGITS } \\
01234\end{array}$ & $x \times \times \times \times 100$ & 0 & $x 4$ \\
\hline $\begin{array}{l}\text { DISPLAY DIGITS } \\
012345\end{array}$ & 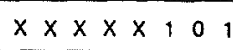 & 1 & $\times 5$ \\
\hline $\begin{array}{l}\text { DISPLAY DIGITS } \\
0123456\end{array}$ & $\times \times \times \times \times \times 110$ & 0 & $x_{6}$ \\
\hline $\begin{array}{l}\text { DISPLAY DIGITS } \\
01234567\end{array}$ & 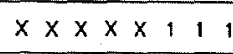 & 1 & $x 7$ \\
\hline
\end{tabular}

- See "Scan-Limit Register" text for application.

Table 9. Maximum Segment Current for 1, 2 or 3 Digit Dlsplays

\begin{tabular}{|cc|}
\hline $\begin{array}{c}\text { NUMBER OF DIGTS } \\
\text { DISPLAYED }\end{array}$ & $\begin{array}{c}\text { MAXIMUM SEGMENT } \\
\text { CURENT }\end{array}$ \\
\hline 1 & $10 \mathrm{~mA}$ \\
\hline 2 & $20 \mathrm{~mA}$ \\
\hline 3 & $30 \mathrm{~mA}$ \\
\hline
\end{tabular}

Table 10. Display-Test Register Format (Address (HeX) $=\mathrm{XF}$ )

\begin{tabular}{|lccccccccc|}
\hline & DE & D6 & D5 & D4 & D3 & D2 & D1 & D0 \\
\hline NORMAL OPERATION & $\times$ & $\times$ & $\times$ & $\times$ & $\times$ & $\times$ & $\times$ & 0 \\
\hline DISPLAY TEST MODE & $\times$ & $\times$ & $\times$ & $\times$ & $\times$ & $\times$ & $\times$ & 1 \\
\hline
\end{tabular}

Note: The MAX7219 remains in display-test mode (all LEDs on) until the display-test register is reconfigured for normal operation.

No.Op Register

The no-op register is used when cascading MAX7219s. Connect all devices' LOAO inputs together and connect DOUT to DIN on adjacent MAX7219s. DOUT is a CMOS logic level output that easily drives DIN of a successively cascaded MAX7219. Refer to the "Serial Addressing Modes" section for detailed information on seria inputoutput timing. For example, if four MAX7219s are cascaded, then to write to the fourth chip, send the desired 16-bit word, followed by three no-op codes (hex $X O X X$, see Table 2). Whenload goes high, data is latched in all devices. The first three chips receive no-op commands, and the fourth receives the intended data. 


\section{Serially Interfaced, 8-Digit LED Display Driver}

\section{Applications Information Supply Bypassing and Wiring}

To minimize power-supply ripple due to the peak digit driver currents, connect a $10 \mu \mathrm{F}$ electrolytic and a $0.1 \mu \mathrm{F}$ ceramic capacitor between $V_{+}$and GND as close to the device as posibl. The MAX7210 should be placed in close proximity to the LED display, and connections should be kept as short as possible to minimize the effects of wiring inductance and electromagnetic interFerence. Also, both GND pins must be connected to ground.

\section{Selecting Rser Resiator and Using} Extemai Drivers

The current per segment is approximately 100 times the current in ISET. To select RSET, see Table 11. The MAX7219's maximum allowable segment current is $40 \mathrm{~mA}$. For an LED forward voltage drop of 2.5V, RSE must be greater than 9.53k. For segment current levels above the MAX7219 limits, external drivers will be needed. In this application, the MAX7219 serves as only a controller for other high-current drivers or transistors. Therefore, to conserve power in the MAX7219, use RSET $=47 \mathrm{k}$ when using external current sources as segment drivers.

The example in Figure 2 uses the MAX7219's segment drivers, a MAX333 single-pole double-throw analog switch, and external transistors to drive $4.0^{\circ}$ AND4107SCL common-cathodo displays. The 5.6V zener diode has been added in serles with the decimal point LED because the decimal point LED forward voltpoin 4 ED because he decim selins We age is typically 4.2V, While for all ofther segments the LED forward voltage is typically $8 \mathrm{~V}$. Note that since external transistors are used to sink current (DIG 0 and DIG 1 are used as logic switches), peak segment currents of $40 \mathrm{~mA}$ are allowed even though only two digits are displayed. In applications where the MAX7219's digit drivers are used to sink ourtent and fewer than four digits an pleyed, seo

Table 11. RSET vs. Segment Current and LED Forward Voltage

\begin{tabular}{|c|c|c|c|c|c|}
\hline \multirow[b]{2}{*}{$\begin{array}{l}\text { ISEg } \\
\text { (mA) }\end{array}$} & \multicolumn{5}{|c|}{ VLED $M$} \\
\hline & 1.5 & 2 & 2.5 & 3 & 3.5 \\
\hline 40 & 11.3 & 10.4 & 9.8 & 8.9 & 7.8 \\
\hline 30 & 16.3 & 15 & 14 & 12.9 & 11.4 \\
\hline 20 & 26.2 & 24.6 & 22.8 & 20.9 & 18.6 \\
\hline 10 & 60.1 & 56 & 51.7 & 47 & 41.9 \\
\hline
\end{tabular}

able segment current. RSET must be selected accordingly (see Table 11).

Refer to the "Power Dissipation" section to calculate acceptable limits for ambient temperature, segment current, and the LED forward-voltage drop.

Table 12. Package Thermal Resistance Data

\begin{tabular}{|lc|}
\hline PACKAGE & THERMAL RESISTANCE \\
\hline$(\Theta \mathrm{JA})$ \\
\hline 24 Narrow DIP & $+75^{\circ} \mathrm{CW}$ \\
24 Wide SO & $+85^{\circ} \mathrm{CN}$ \\
24 CERDIP & $+60^{\circ} \mathrm{CW}$ \\
\hline Maximum Junction Temperature $\left(\mathrm{T}_{\mathrm{J}}\right)=+150^{\circ} \mathrm{C}$ \\
\hline Maximum Ambient Temperature $\left(\mathrm{T}_{\mathrm{A}}\right)=+85^{\circ} \mathrm{C}$ \\
\hline
\end{tabular}

\section{Computing Power Dlssipation}

The upper limit for power dissipation (PD) for the MAX7219 is determined from the following equation:

$\mathrm{PD}=(\mathrm{V}+\times 8 \mathrm{~mA})+(\mathrm{V}+\mathrm{VLEO})($ DUTY $\times$ ISEG $\times \mathrm{N})$ where:

$V_{+}=$Supply Voltage

DUTY = Duty Cycle set by intensity register

$N=$ number of segments driven (worst case is 8)

$V_{L E D}=\mathrm{LED}$ forward voltage

ISEG = Segment Current set by RSET

Dissipation Example:

ISEG $=40 \mathrm{~mA}, \mathrm{~N}=8, \mathrm{DUTY}=31 / 32, \mathrm{~V}_{\mathrm{LED}}=1.8 \mathrm{~V}$ at $40 \mathrm{~mA}, V_{+}=5.25 \mathrm{~V}$

$P D=5.25 V(8 m A)+(5.25 V-1.8 V)(31 / 32 \times 40 \mathrm{~mA} \times 8)$ $=1.11 \mathrm{~W}$

Thus, for a CERDIP package $\left(\theta, 1 A=+60 C^{*}\right.$ N W rom table 12$)$ the maximum allowed ambient temperature $T A$ is given by:

$T_{J \max }=T_{A}+P D \times \theta_{\text {JA }}$

$+150^{\circ} \mathrm{C}=\mathrm{TA}+1.11 \mathrm{~W} \mathrm{X}+60^{\circ} \mathrm{C} \mathrm{W}$

$T_{A}=+83.4^{\circ} \mathrm{C}$

Cascading Drivers

The example in Figure 3 drives 16 digits using a 3-wire $\mu \mathrm{P}$ interface. If the number of digits is not a multiple of 8 , set both drivers' scan-limit registers to the same number so one display will not appear brighter than the other. For example, if 12 digits are needed, use 6 digits per display

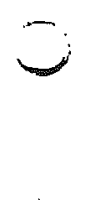

(

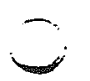

(1) 


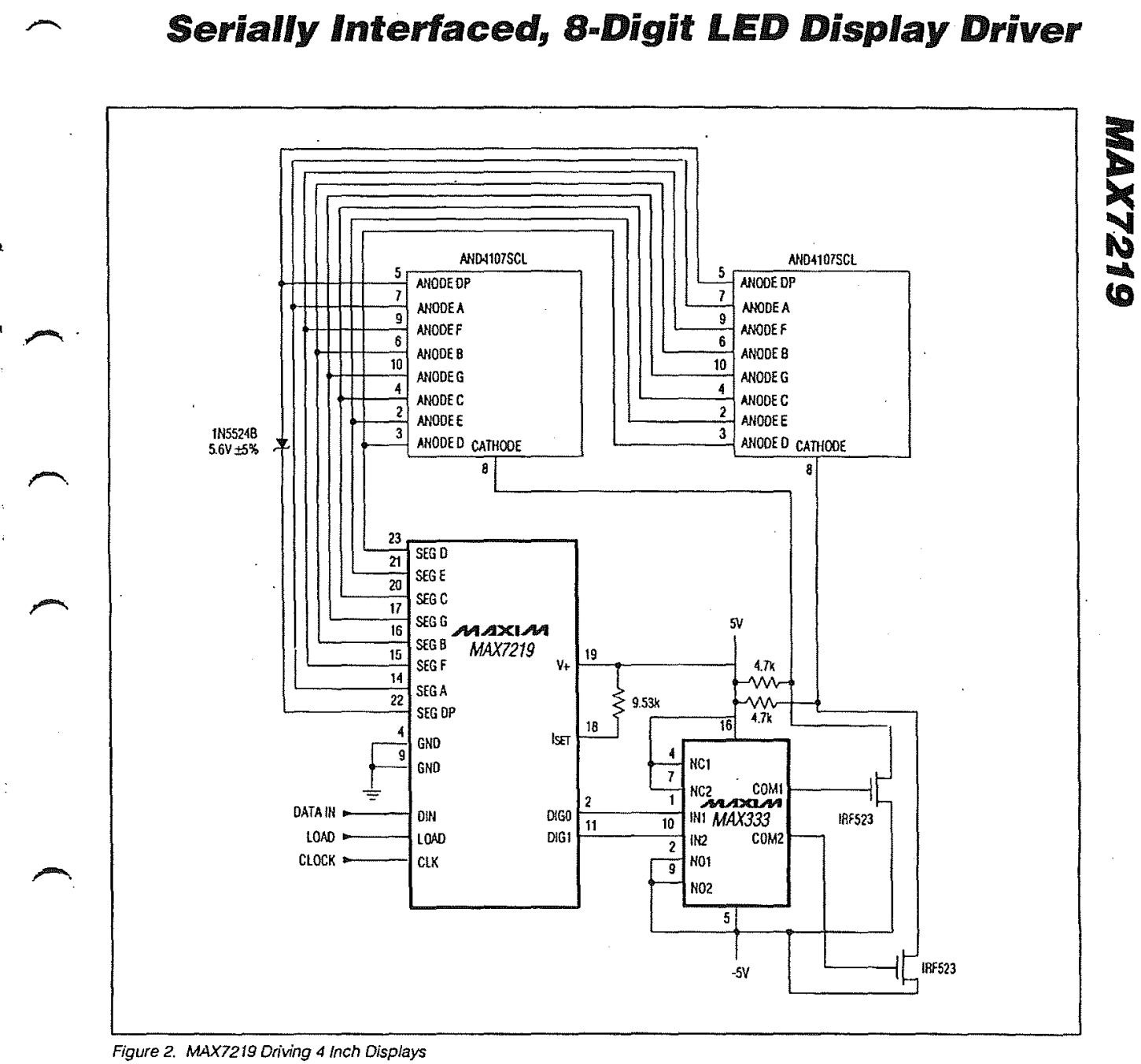

Figure 2. MAX7219 Driving 4 Inch Displays 


\section{Serially Interfaced, 8-Digit LED Display Driver}

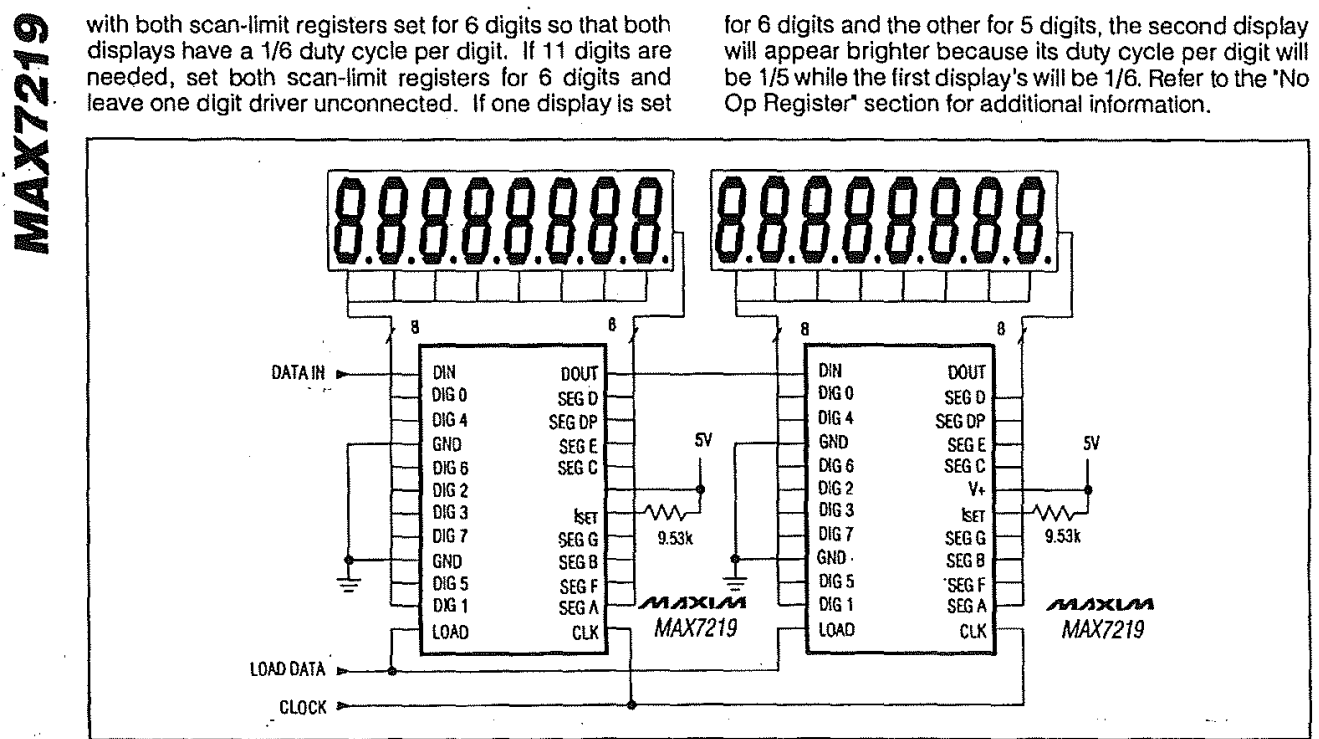

Figure 3. Cascading MAX7219s to drive 16 7-segment LED digits. 
Serially Interfaced, 8-Digit LED Display Driver

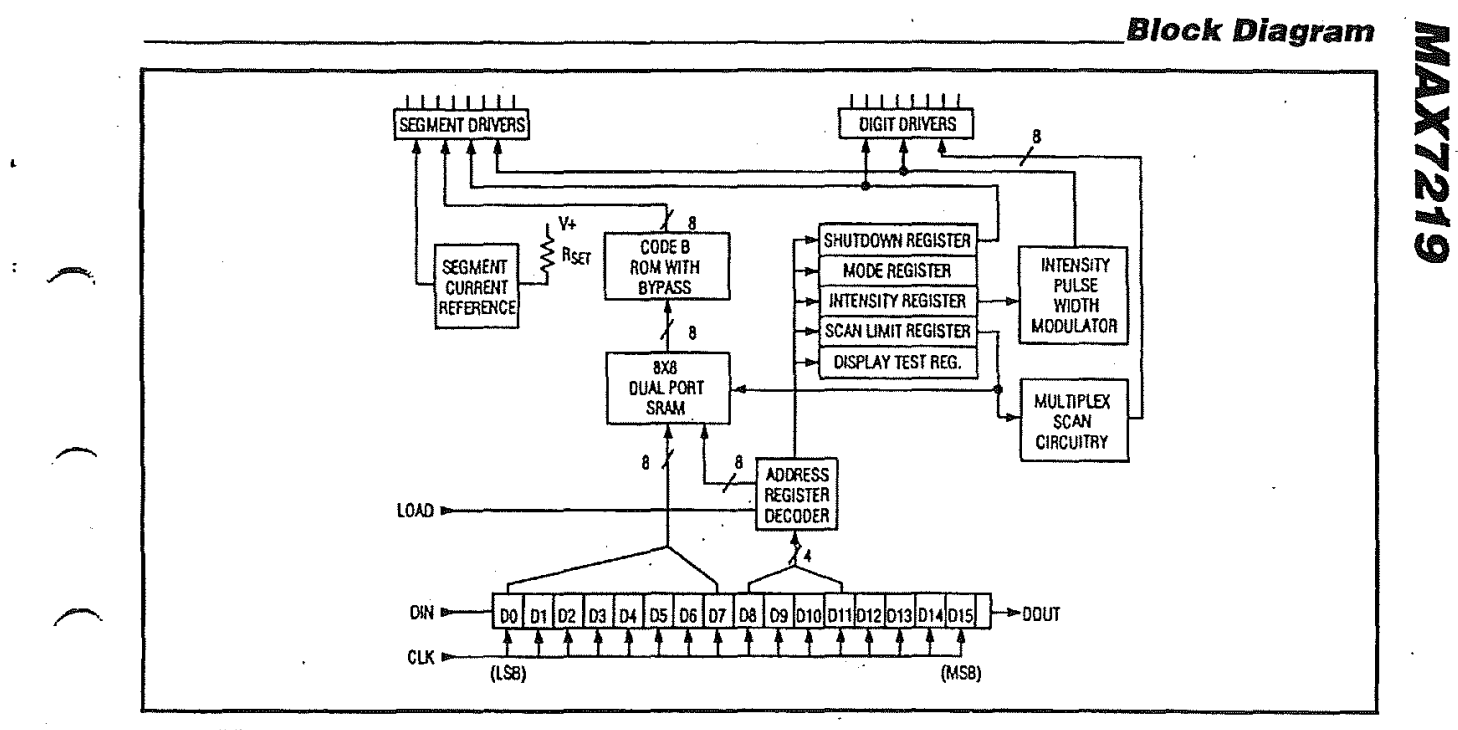

Chip Topography

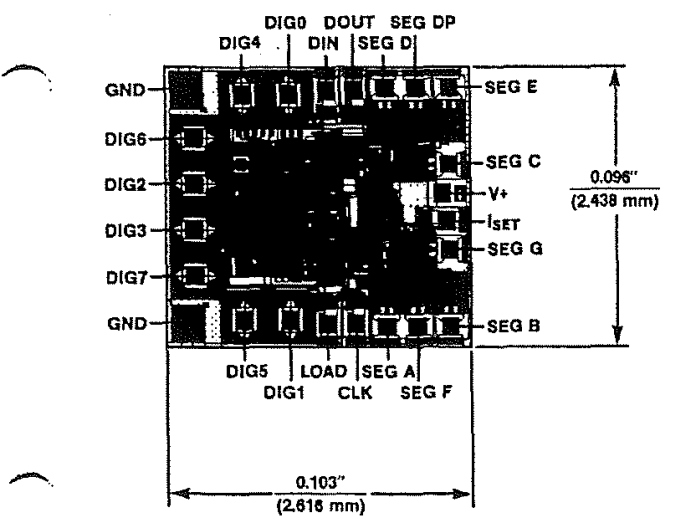




\section{Appendix E. Battery Monitor Software}

